\title{
Development and Testing of a Passive Ankle Exoskeleton
}

\author{
Scott Pardoel
}

Thesis Submitted In Partial Fulfilment of the Requirements for the Masters of Applied Science in Biomedical Engineering

Ottawa-Carleton Institute for Biomedical Engineering Department of Mechanical Engineering

Faculty of Engineering

University of Ottawa

(C) Scott Pardoel, Ottawa, Canada, 2017 


\section{Abstract}

Aging is accompanied by a deterioration of physical abilities. For some this limits their mobility and thus their quality of life. Exoskeletons are a class of walking assist device that help reduce the effort required to walk. Currently, powered exoskeletons suffer from short battery life and thus limited usefulness. This thesis presents the design, fabrication, and testing of a novel unpowered ankle exoskeleton to assist normal walking over long distances. The design incorporates a Pneumatic Artificial Muscle (PAM) inflated and used as a passive air spring. To predict the behaviour of the PAM in this distinct application, a distinct dynamic model was developed to include the biaxial stress in the bladder as well as a polytropic gas assumption. Experimental testing was used to validate the model and indicated that the addition of the bladder stress enhanced the performance of the force prediction at low pressure but had negligible impact on the model at higher pressures. The experimental testing also showed that the temperature of the gas inside the PAM varies very slightly during passive elongation cycles, thus, validating an isothermal assumption. Once fabricated, the exoskeleton was tested in human walking trials.

Electromyography results showed that the exoskeleton was able to reduced the muscular activation activation of the Soleus muscle, however the results also included a significant reduction in the angular range of motion of the ankle. This is thought to be attributed to an insufficient acclimatization period during the human testing. Furthermore, due to an improper fit of the exoskeleton, the clutch mechanism did not operate as designed, leading to a reduced range of motion of the ankle. The device demonstrated its ability to reduce the effort of the calf muscles during walking, however, further refinements of the device fitting and clutch mechanism are required. 


\section{Acknowledgements}

I would like to thank to Dr. Doumit for supervising and reviewing the work that was done during this project. His guidance and advice has been indispensable.

Thank you to the University of Ottawa Maker Space for the use of their 3D printers. Special thanks to Danielle Taillon for her expert advice and invaluable technical support.

I would also like to thank Laurent Levesque as well as my other lab mates for sharing their knowledge and helping to address unexpected problems as they arose. 


\section{Contents}

1 Introduction $\quad 1$

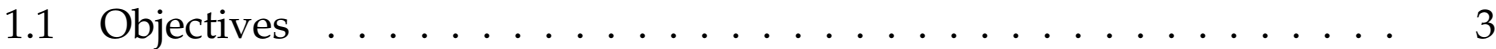

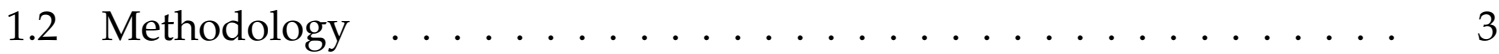

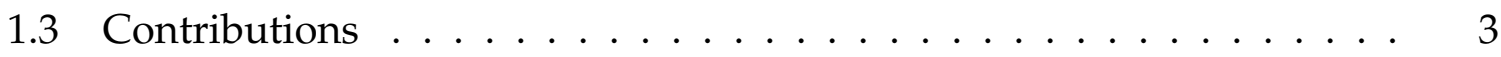

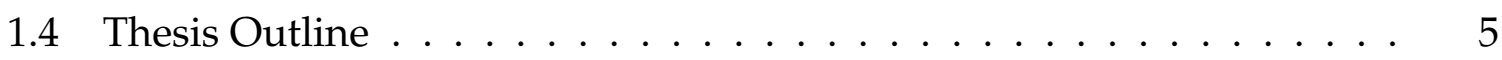

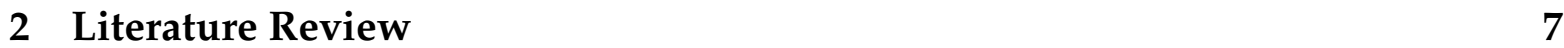

2.1 Walking Assist Devices . . . . . . . . . . . . . . . . 8

2.1 .1 Assistive motion . . . . . . . . . . . . . . 8

2.1 .2 Assistive devices .................. . . 8

2.1.3 Hybrid - Minimally Powered Devices . . . . . . . . . . . . . . 12

2.1 .4 Passive devices . . . . . . . . . . . . . . . . 16

2.2 The Pneumatic Artificial Muscle . . . . . . . . . . . . . . . 20

2.2.1 Existing PAM Models ................ 21

3 PAM Modelling and Simulation $\quad 26$

3.1 PAM modelling . . . . . . . . . . . . . . . . 26

3.1 .1 Force model . . . . . . . . . . . . . . . . 26

3.1 .2 Bladder Stress . . . . . . . . . . . . . . . . . . 28

3.1 .3 Pressure model . . . . . . . . . . . . . 38

3.1 .4 Temperature model . . . . . . . . . . . . 38

3.1 .5 Friction . . . . . . . . . . . . . . . 39

3.1.6 Simulation ....................... 40

3.2 PAM experimental testing . . . . . . . . . . . . . . . . . 41

3.2.1 Testing Methodology . . . . . . . . . . . . . . 42

3.2.2 Model Validation . . . . . . . . . . . . . . . 46

4 Design, Modelling, Fabrication and Mechanical Testing 56 
4.1 Design Parameters . . . . . . . . . . . . . . . . . . . . . 57

4.2 Proposed design . . . . . . . . . . . . . . . . . 58

4.3 Kinematic Simulation . . . . . . . . . . . . . . . . . . . . . . 60

4.4 Timing Mechanism and operational phases . . . . . . . . . . . 66

4.5 Manufacturing . . . . . . . . . . . . . . . . . . 70

4.6 Testing Procedures . . . . . . . . . . . . . . . . . 73

4.6.1 Experimental Setup . . . . . . . . . . . . . . . . . 73

4.6 .2 Testing Parameters . . . . . . . . . . . . . . . 74

4.6 .3 Data processing . . . . . . . . . . . . . . . . 75

4.7 Results . . . . . . . . . . . . . . . . . 77

5 Human testing $\quad 83$

5.1 Ethics Approval and Recruitment . . . . . . . . . . . . . . . . . 84

5.1 .1 Participants . . . . . . . . . . . . . . . 84

5.2 Testing Protocols . . . . . . . . . . . . . . . . . . . . . 84

5.2 .1 Motion Capture . . . . . . . . . . . . . . . . . . 84

5.2 .2 Electromyography $(\mathrm{EMG}) \ldots \ldots \ldots \ldots$. . . . . . . . 86

5.2 .3 Experimental Protocol . . . . . . . . . . . . . . . . 87

5.2 .4 Data Analysis . . . . . . . . . . . . . . . . . . . . 9 90

5.3 Results . . . . . . . . . . . . . . . . . . . . . 92

5.3.1 Motion Capture Results . . . . . . . . . . . . . . . . . . 92

5.3.2 EMG Results . . . . . . . . . . . . . . . . . . . . . . . . 97

5.4 Limitations and Sources of Error _ . . . . . . . . . . . . . . 103

5.4.1 Limitations of the Exoskeleton Device . . . . . . . . . . . . 103

5.4.2 Limitations of Methodology and testing protocols . . . . . . . 103

6 Conclusions and Recommendations 105

6.1 Conclusion . . . . . . . . . . . . . . . . . . . . . . . . . . 106

6.2 Future Work . . . . . . . . . . . . . . . . . . . . . 107

Appendix A Human locomotion and fundamentals of gait 116

A.1 Human Locomotion . . . . . . . . . . . . . . . . . . . . . . . 116

A.1.1 Physiology Anatomy of the Lower Limbs . . . . . . . . . . . 116

A.1.2 Anthropometric Data . . . . . . . . . . . . . 122

A.1.3 Fundamentals of Gait . . . . . . . . . . . . . . . . 123

A.1.4 Ground Reaction Forces . . . . . . . . . . . . . . . 126

A.1.5 Gait Energy Analysis . . . . . . . . . . . . . . . . . . . 128 
C.1 PAM specifications . . . . . . . . . . . . . . . . . . . . . . 140

C.2 PAM validation results . . . . . . . . . . . . . . . . . . . . 143

C.3 PAM RMSE results . . . . . . . . . . . . . . . . . . . . . . 155

Appendix D Mechanical testing results 159

D.1 Comparison of 25\% of ideal spring force vs PAM . . . . . . . . . . . 159

D.2 Comparison of ideal spring force vs PAM, with percentage of ideal spring adjusted to fit PAM . . . . . . . . . . . . . . . 162

D. 3 Comparison of $25 \%$ of biological ankle torque (for $86 \mathrm{~kg}$ individual) vs torque produced by exoskeleton . . . . . . . . . . . . 165

D.4 Comparison of biological ankle torque (for $86 \mathrm{~kg}$ individual) vs torque produced by exoskeleton with percentage of biological ankle torque adjusted to fit PAM with percentage of biological torque adjusted to fit exoskeleton . . . . . . . . . . . . . . . . . . . . 168

$\begin{array}{lll}\text { Appendix E Human Testing Results for participant 1 } & 171\end{array}$

E.1 Kinematic results for participant $1 \ldots \ldots \ldots \ldots 171$

E.1.1 PAM $70 \mathrm{kPa} \ldots \ldots \ldots \ldots \ldots$

E.1.2 PAM $40 \mathrm{kPa} \ldots \ldots \ldots \ldots \ldots \ldots \ldots$

E.1.3 Elastic Spring Element . . . . . . . . . . . . . . . . . . 177

E.1.4 No Spring Element . . . . . . . . . . . . . . . . . . . . . . . . 180

E.1.5 Baseline Walking (No Exo) _ . . . . . . . . . . . . . . . 183

E.2 Participant 1 EMG walking trials results . . . . . . . . . . . 186

$\begin{array}{lll}\text { Appendix F } & \text { Ethics Certificate } & 194\end{array}$

Appendix G MatLab Simulations and data processing code 197

G.1 Simulink Simulation . . . . . . . . . . . . . . . . . . . . . . . 197

G.2 Comparison of PAM Simulation and Experimental Testing Script . . . 198

G.3 Kinematic Simulation of Exoskeleton Script . . . . . . . . . . . . . . . 201

G.4 Mechanical Testing Analysis Script . . . . . . . . . . . . . . . . . . . 209

G.5 Human Testing EMG Processing Script . . . . . . . . . . . . . 213 


\section{List of Figures}

1.1 PAM prototype a) Pressurized state b) Deflated state $\ldots . . . \ldots 2$

1.2 Photograph of the final prototype . . . . . . . . . . . . 5

2.1 Peak values for joint moments at the knee and ankle joints. Data are presented as mean (bars) and standard deviation (vertical lines) for the group ( $\mathrm{n}=11)$. a-significantly different from comfortable walking $(\mathrm{P}<0.05)$; b-significantly different from fast walking $(\mathrm{P}<0.05)$; c-significantly different from slow running $(\mathrm{P}<0.05)$; d-significantly different from running $(\mathrm{P}<0.05)$. Image and caption taken from $[14] \ldots \ldots \ldots$

2.2 The Honda Walking Assist Device with Stride Management System [17] 9

2.3 ReWalk 6 exoskeleton suit by ReWalk Robotics [18] . . . . . . . . . . 10

2.4 Photograph of the ankle exoskeleton prototype [9] . . . . . . . . . . . 11

2.5 A) Prototype energy recycling device B) the main mechanical components of the device C) Position and action of the device during walking. The heel spring is compressed during the energy capture and energy storage phase. The energy return phase corresponds to the release of the stored energy in the form of a plantar flexion moment. [20] . . . 13

2.6 The AMP foot $2.0[23] \ldots \ldots$. . . . . . . . . . . . . . 14

2.7 Illustration of the geometric locking mechanism of the AMP 2.0 a) locked position b) unlocked [24] . . . . . . . . . . . . . . . . . . 14

2.8 Schematic representation of combination pneumatic spring and leaf spring prosthesis $[27] \ldots \ldots \ldots \ldots \ldots$

2.9 Thermoplastic ankle foot orthosis by Ottobock [29] . . . . . . . . . 17

2.10 A) Simplified steps of a counter movement jump with hands on hips in the sagittal plane. B) Elastic storage and release mechanism of the passive-elastic ankle exoskeleton. C) Center of Mass (CoM) mechanical power during the counter movement jump. CoM work is shown by the shaded area below the curve. Image and description taken from [32] . 
2.11 Illustration of the implementation of the Smart-Clutch mechanism. Blue line shows the normal ankle joint profile for walking. Image taken from [33]

2.12 PAM prototype a)Pressurized state $b$ )Deflated state $\ldots \ldots \ldots 20$

3.1 General PAM structure . . . . . . . . . . . . . . . . . . . . 27

3.2 Geometrical properties of the braided mesh that reflects the muscle middle section. Image and caption taken from [35] . . . . . . . . . .

3.3 Photograph of the biaxial testing machine. The sample is suspended in midair between the four instrumented arms. . . . . . . . . . .

3.4 Photograph of the biaxial testing of a butyl rubber sample at 6 stages

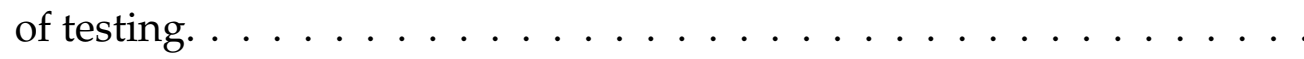

3.5 Graph of the stress vs strain relationship of the butyl rubber sample 5. Strain ratio of $0.93 /-0.278$, cycle duration of $30 \mathrm{~s}$. A) Longitudinal Stress B) Circumferential Stress . . . . . . . . . . . . . . .

3.6 Graph of the stress vs strain relationship of the fifth cycle of the butyl rubber sample 5, and the sixth order polynomial curve that was fit to the data. Strain ratio of $0.93 /-0.278$, cycle duration of 30 s. A) Longitudinal Stress B) Circumferential Stress . . . . . . . . . . . .

3.7 Comparison of the fitted curves for samples 2 and 3. Strain ratio of 0.93 / -0.278 , cycle duration of $30 \mathrm{~s}$ and $60 \mathrm{~s}$. A) Longitudinal Stress B) Circumferential Stress $\ldots \ldots \ldots \ldots \ldots \ldots$

3.8 Comparison of the fitted curves for samples 2 and 6. Sample 2 was tested at strain ratio of $-0.278 / 0.93$, sample 6 was tested at strain ratio of $-0.29 / 0.90$. A) Longitudinal Stress B) Circumferential Stress . . . . 37

3.9 PAM simulation block diagram . . . . . . . . . . . . . . . . 40

3.10 PAM prototype in the Instron universal testing machine, model 4482 . 41

3.11 PAM testing setup in Instron linear testing machine. The wires feeding into the muscle are connected to the temperature sensor. The pressure transducer is also visible at the bottom of the photograph. . . . . . .

3.12 Comparison of the pressure results for the experimental and simulation without bladder stress model. The muscle was inflated to $207 \mathrm{kPa}$ and tested at an elongation of $90 \%$.

3.13 Comparison of the pressure results for the experimental and simulation with bladder stress. The muscle was inflated to $207 \mathrm{kPa}$ and tested at an elongation of $90 \%$. 
3.14 Comparison of the force results for the experimental and simulation with bladder stress, at $276 \mathrm{kPa}$ at elongation of $95 \%$. . . . . . . . . 48

3.15 Comparison of the force results for the experimental and simulation without bladder stress model, at $276 \mathrm{kPa}$ at elongation of $95 \%$. . . .

3.16 Comparison of the force results for the experimental and simulation with bladder stress model included, at $69 \mathrm{kPa}$. . . . . . . . . . . 50

3.17 Comparison of the force results for the experimental and simulation without bladder stress model, at $69 \mathrm{kPa}$. . . . . . . . . . . . . 50

3.18 Comparison of the pressure results for the experimental and simulation with bladder stress, at $55 \mathrm{kPa} . \ldots \ldots \ldots \ldots$

3.19 Comparison of the pressure results for the experimental and simulation without bladder stress model, at $55 \mathrm{kPa}$. . . . . . . . . . . . . 52

3.20 Internal gas temperature for cyclical testing elongations of $80 \% 90 \%$ and $95 \%$ for pressure of $207 \mathrm{kPa}(\mathrm{A}, \mathrm{B}, \mathrm{C})$, and $276 \mathrm{kPa}(\mathrm{D}, \mathrm{E}, \mathrm{F}) \ldots \ldots$

3.21 Comparison of the rise of the internal temperature predicted by the simulation and the measured temperature for the $276 \mathrm{kPa} 95 \%$ elongation scenario. . . . . . . . . . . . . . . . .

4.1 Photograph of the completed exoskeleton prototype . . . . . . . . . 59

4.2 Simplified layout of the lower limbs used during kinematic simulations. 60

4.3 Illustration of the variation of the effective moment arm of the device during stance phase. The direction of the spring force relative to the ankle joint. The dotted line represents the perpendicular distance between the force and the rotation point. . . . . . . . . . .

4.4 Illustration of the variation of the effective moment arm of the device. This is the perpendicular distance between the line of action of the force and the pivot point. . . . . . . . . . . . . . . .

4.5 A) illustrates the length of the spring element during the gait cycle. The length of the spring varies approximately between 25 and $30 \mathrm{~cm}$. B) illustrates the net elongation of the spring during the gait cycle . . .

4.6 Comparison of torque profiles. Both linear and PAM springs are assumed to be used with the exoskeleton device. The magnitudes have been normalized to $25 \%$ of the maximum natural ankle torque . . . . .

4.7 Force vs Elongation profile of the theoretically ideal spring if the device were to replicate the normal torque profile of the natural ankle joint for a person weighing $86 \mathrm{~kg} \ldots \ldots \ldots \ldots \ldots$ 
4.8 Force vs Elongation profile of a PAM with 2 fiber revolutions and an initial pressure of $138 \mathrm{kPa} \ldots \ldots \ldots \ldots$

4.9 Illustration of the engagement and disengagement of the timing mech-

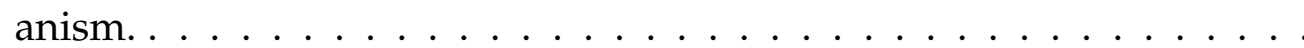

4.10 Illustration of the complete timing mechanism. The Back Plate, Ratchet Gear plate as well as the Engagement arm are all free to rotate independently around the Ankle joint . . . . . . . . . . . . .

4.11 Illustration of the entire exoskeleton model showing the position of the timing mechanism as well as the pre-tension screw. . . . . . . . . . .

4.12 Illustration of the engagement and disengagement of the timing mechanism throughout the gait cycle.(A) shows the movement of the mechanism from the disengaged to the engaged state. (B) shows the engaged state of the mechanism (C) shows the movement of the mechanism from the engaged to the disengaged state . . . . . . . . . .

4.13 Photograph of the first complete prototype. White pieces were 3D printed from ABS. Upright pieces are made from aluminum . . . . . 71

4.14 Photograph of the complete prototype. . . . . . . . . . . . . 72

4.15 Photograph of the mechanical testing setup. The $\mathrm{L}$ bracket secures the foot plate while the shank section of the device is free to rotate. . . . .

4.16 Illustration of the side view of the device with member lengths and

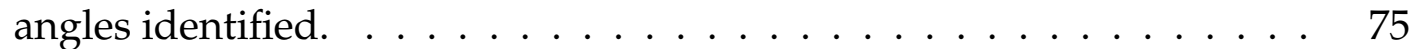

4.17 Free body diagram of the shank section of the ankle exoskeleton device. Point $\mathrm{A}$ is the attachment point of the PAM and the shank cuff. Values $A_{x}$ and $A_{y}$ are the horizontal and vertical distances between the ankle joint and point A. Angle $\gamma$ defines the position of the PAM with respect to horizontal . . . . . . . . . . . . . . . .

4.18 Free body diagram of the heel bar section of the ankle exoskeleton device. Point $\mathrm{B}$ is the attachment point of the pneumatic muscle. Angle $\alpha$ is the angle between the heel bar and vertical. During testing this angle was kept constant at $5^{\circ} \ldots \ldots \ldots \ldots \ldots \ldots \ldots$

4.19 Force vs elongation graph of the mechanical testing of the PAM compared with the theoretically ideal spring. The PAM is inflated to $70 \mathrm{kPa}$ and the vertical displacement of the Instron cross head is $5 \mathrm{~cm}$. . . . . 
4.20 Joint torque vs percentage of gait cycle of the mechanical testing of the PAM compared with the theoretically ideal spring. The PAM was inflated to $70 \mathrm{kPa}$ and the vertical displacement of the Instron cross head was $5 \mathrm{~cm}$

4.21 Force vs elongation graph of the mechanical testing of the PAM compared with the theoretically ideal spring. The PAM was inflated to 48 $\mathrm{kPa}$ and the vertical displacement of the Instron cross head was $8 \mathrm{~cm}$.

4.22 Joint torque vs percentage of gait cycle of the mechanical testing of the PAM compared with the theoretically ideal spring. The PAM was inflated to $48 \mathrm{kPa}$ and the vertical displacement of the Instron cross head was $8 \mathrm{~cm}$.

4.23 Joint torque vs percentage of gait cycle of the mechanical testing of the PAM compared with the theoretically ideal spring. The PAM is inflated to $48 \mathrm{kPa}$ and the vertical displacement of the Instron cross head is 8 $\mathrm{cm}$. The ideal spring force is set to $50 \%$ of the natural biological torque

5.1 Photograph of the legs of a participant once the exoskeleton, the EMG sensors, and the reflective markers were placed . . . . . . . . . . .

5.2 Photograph of the placement of the EMG sensors on the legs of a participant wearing the exoskeleton $\ldots \ldots \ldots \ldots \ldots$

5.3 Ankle angle for baseline walking trial (without exoskeleton) A) Right leg, B) Left leg . . . . . . . . . . . . . . . . . . . . . .

5.4 Walking trial ankle angle for exoskeleton with PAM inflated to $70 \mathrm{kPa}$ for participant 1. A) Right leg (wearing exoskeleton), B) Left leg (without exoskeleton) . . . . . . . . . . . . . . . .

5.5 Walking trial spring length for exoskeleton with PAM inflated to $70 \mathrm{kPa}$ for participant $1 \ldots \ldots \ldots \ldots \ldots \ldots$

5.6 EMG output of the baseline walking trial. The image shows the recorded muscle activation of the six specific muscles during the gait cycle. A) Right Tib Ant, B) Right Gas Med, C) Right Soleus, D) Left Tib Ant, E) Left Gas Med, F) Left Soleus . . . . . . . . . . . . . . . . . . .

5.7 EMG output of the walking trial with the exoskeleton and the PAM inflated to $70 \mathrm{kPa}$. The image shows the recorded muscle activation of the six specific muscles during the gait cycle. A) Right Tib Ant, B) Right Gas Med, C) Right Soleus, D) Left Tib Ant, E) Left Gas Med, F) Left Soleus 98 
5.8 EMG output of the walking trial with the exoskeleton and the PAM inflated to $40 \mathrm{kPa}$. The image shows the recorded muscle activation of the six specific muscles during the gait cycle. A) Right Tib Ant, B) Right Gas Med, C) Right Soleus, D) Left Tib Ant, E) Left Gas Med, F) Left Soleus 99

5.9 Comparison of the muscle activation in the Right Gastrocnemius Medialis during the PAM $70 \mathrm{kPa}, \mathrm{PAM} 40 \mathrm{kPa}$, Elastic, and baseline trials for subject 1 . The exoskeleton was worn on the Right leg . . . . . . . 100

5.10 Comparison of the muscle activation in the Left Gastrocnemius Medialis during the PAM $70 \mathrm{kPa}$, PAM $40 \mathrm{kPa}$, Elastic, and baseline trials for subject 1 . The exoskeleton was worn on the Right leg . . . . . . . . 101

A.1 Anatomical planes used to describe the human body [57] . . . . . . 117

A.2 Movement about the hip, knee, ankle and foot joints. Modified from [58] . . . . . . . . . . . . . . . . . . .

A.3 General anatomy of the lower leg. A) Posterior view of leg muscles B) Anterior view of leg muscles [61] . . . . . . . . . . . . . . 118

A.4 Experimental results illustrating the mechanical power in the shank. MTU is the muscle tendon unit, TT is the tendinous tissues and includes aponeuroses [63] . . . . . . . . . . . . . . . . . . . . . 121

A.5 Various segment lengths expressed has a function of body height [70] . 123

A.6 Illustration of one full stride. Adapted from [71] . . . . . . . . . . . 124

A.7 The Gait cycle showing the division of the stance phase and the swing

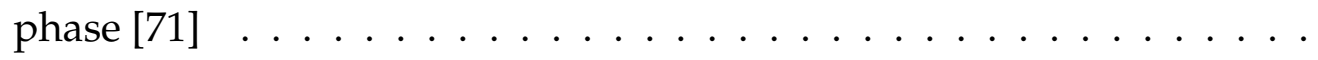

A.8 Gait cycle illustrating different stages and primary muscles active during each phase. The black arrows illustrate the direction of the ground reaction forces during stance phase. During the swing phase these arrows indicate the advancement of the $\operatorname{limb}[72] \ldots \ldots \ldots$

A.9 GRF in function of gait phase of walking modified from [73] . . . . . 127

A.10 Butterfly (or Pedotti) diagram modified from [73] . . . . . . . . . . 128

A.11 Experimental set up. A) shows the participant on an instrumented treadmill wearing a respirometer. B) illustrates how the muscle activity of the Soleus was captured with surface electromyography then the pneumatic muscle was activated to compliment the joint movement. Modified from [10] . . . . . . . . . . . . . . . . . . . . . . . . . . . . . . 129 
A.12 Normalized mean electromyogram vs load condition relative to unloaded walking for the soleus and medial gastrocnemius during midstance (32-52\% stride) (A), the rectus femoris during late stance (57$62 \%$ stride) and early swing (62-67\% stride) (B), and the tibialis anterior and semitendinosus during late swing (90-100\% stride) (C). Values are means for all subjects. EMG magnitudes tended to increase with foot loading in the soleus, medial gastrocnemius, and rectus femoris during stance and the tibialis anterior during swing. Image and caption taken from [75] . . . . . . . . . . . . . . . . .

A.13 Mean net metabolic rate (W·kg-1) vs load mass $(\mathrm{m})$ and location. The increase in net metabolic rate was greatest with foot loads, similar for thigh and shank loads, and least for waist loads. *Significant difference, load location vs waist load. Image and caption taken from [75] $\ldots . .132$

A.14 Graphical illustration of gait data for the hip knee and ankle joints during healthy walking. Values are normalized by body weight A) Joint angles [Degrees] B) Joint Moments [ $\mathrm{N}^{*} \mathrm{~m} / \mathrm{Kg}$ ] C) Joint Power [W/Kg] D) Illustration of active muscles during gait cycle. Modified from [6] .

A.15 The joint power of the ankle and knee of healthy human gait over one full stride, normalized by body weight. Modified from [7] . . . . . .

B.1 A) shows the pressure transducer, B) shows the hose and valve connecting the transducer to the PAM . . . . . . . . . 137

B.2 TMP 36 temperature sensor. The ruler is in $\mathrm{cm} \ldots \ldots \ldots \ldots$

B.3 PAM end caps, A) Normal configuration, one end cap with inflation orifice, the other without. B) Photograph of the PAM end caps showing that both have an orifice for inflation . . . . . . . . . . . . 139

C.1 Force comparison of experimental and simulated results for PAM inflated to $207 \mathrm{kPa}$, tested at $80 \%$ elongation. Bladder stress is not included in this simulation . . . . . . . . . . . . . . .

C.2 Pressure comparison of experimental and simulated results for PAM inflated to $207 \mathrm{kPa}$, tested at $80 \%$ elongation. Bladder stress is not included in this simulation . . . . . . . . . . . . . . . . . 143

C.3 Force comparison of experimental and simulated results for PAM inflated to $207 \mathrm{kPa}$, tested at $80 \%$ elongation. Bladder stress is indeed included in this simulation . . . . . . . . . . . . . . . . . . 144 
C.4 Pressure comparison of experimental and simulated results for PAM inflated to $207 \mathrm{kPa}$, tested at $80 \%$ elongation. Bladder stress is indeed included in this simulation . . . . . . . . . . . . . . . .

C.5 Force comparison of experimental and simulated results for PAM inflated to $207 \mathrm{kPa}$, tested at $90 \%$ elongation. Bladder stress is not included in this simulation . . . . . . . . . . . . . . . . . . . 145

C.6 Pressure comparison of experimental and simulated results for PAM inflated to $207 \mathrm{kPa}$, tested at $90 \%$ elongation. Bladder stress is not included in this simulation . . . . . . . . . . . . . . . .

C.7 Force comparison of experimental and simulated results for PAM inflated to $207 \mathrm{kPa}$, tested at $90 \%$ elongation. Bladder stress is indeed included in this simulation . . . . . . . . . . . . . . .

C.8 Pressure comparison of experimental and simulated results for PAM inflated to $207 \mathrm{kPa}$, tested at $90 \%$ elongation. Bladder stress is indeed included in this simulation . . . . . . . . . . . . . . . . . 146

C.9 Force comparison of experimental and simulated results for PAM inflated to $207 \mathrm{kPa}$, tested at $95 \%$ elongation. Bladder stress is not included in this simulation . . . . . . . . . . . . . . . . . . . . 147

C.10 Pressure comparison of experimental and simulated results for PAM inflated to $207 \mathrm{kPa}$, tested at 95\% elongation. Bladder stress is not included in this simulation . . . . . . . . . . . . . . . . . 147

C.11 Force comparison of experimental and simulated results for PAM inflated to $207 \mathrm{kPa}$, tested at $95 \%$ elongation. Bladder stress is indeed included in this simulation . . . . . . . . . . . . .

C.12 Pressure comparison of experimental and simulated results for PAM inflated to $207 \mathrm{kPa}$, tested at $95 \%$ elongation. Bladder stress is indeed included in this simulation . . . . . . . . . . . . . . . . . . 148

C.13 Force comparison of experimental and simulated results for PAM inflated to $276 \mathrm{kPa}$, tested at $80 \%$ elongation. Bladder stress is not included in this simulation . . . . . . . . . . . . . . . . . . . . . . . 149

C.14 Pressure comparison of experimental and simulated results for PAM inflated to $276 \mathrm{kPa}$, tested at $80 \%$ elongation. Bladder stress is not included in this simulation . . . . . . . . . . . . . . . .

C.15 Force comparison of experimental and simulated results for PAM inflated to $276 \mathrm{kPa}$, tested at $80 \%$ elongation. Bladder stress is indeed included in this simulation . . . . . . . . . . . . . . . . . . 150 
C.16 Pressure comparison of experimental and simulated results for PAM inflated to $276 \mathrm{kPa}$, tested at $80 \%$ elongation. Bladder stress is indeed included in this simulation . . . . . . . . . . . . . . . . .

C.17 Force comparison of experimental and simulated results for PAM inflated to $276 \mathrm{kPa}$, tested at $90 \%$ elongation. Bladder stress is not included in this simulation . . . . . . . . . . . . . . .

C.18 Pressure comparison of experimental and simulated results for PAM inflated to $276 \mathrm{kPa}$, tested at $90 \%$ elongation. Bladder stress is not included in this simulation . . . . . . . . . . . . . . . . .

C.19 Force comparison of experimental and simulated results for PAM inflated to $276 \mathrm{kPa}$, tested at $90 \%$ elongation. Bladder stress is indeed included in this simulation . . . . . . . . . . . . . . . . . 152

C.20 Pressure comparison of experimental and simulated results for PAM inflated to $276 \mathrm{kPa}$, tested at $90 \%$ elongation. Bladder stress is indeed included in this simulation . . . . . . . . . . . . . . .

C.21 Force comparison of experimental and simulated results for PAM inflated to $276 \mathrm{kPa}$, tested at $95 \%$ elongation. Bladder stress is not included in this simulation . . . . . . . . . . . . .

C.22 Pressure comparison of experimental and simulated results for PAM inflated to $276 \mathrm{kPa}$, tested at $95 \%$ elongation. Bladder stress is not included in this simulation . . . . . . . . . . . . . . .

C.23 Force comparison of experimental and simulated results for PAM inflated to $276 \mathrm{kPa}$, tested at $95 \%$ elongation. Bladder stress is indeed included in this simulation . . . . . . . . . . . . .

C.24 Pressure comparison of experimental and simulated results for PAM inflated to $276 \mathrm{kPa}$, tested at $95 \%$ elongation. Bladder stress is indeed included in this simulation . . . . . . . . . . . . . .

D.1 Mechanical testing results of the force vs elongation with the PAM inflated to $70 \mathrm{kPa}$ and with a vertical displacement of $5 \mathrm{~cm} \ldots \ldots .$.

D.2 Mechanical testing results of the force vs elongation with the PAM inflated to $70 \mathrm{kPa}$ and with a vertical displacement of $6 \mathrm{~cm} \ldots \ldots$

D.3 Mechanical testing results of the force vs elongation with the PAM inflated to $55 \mathrm{kPa}$ and with a vertical displacement of $7 \mathrm{~cm} \ldots \ldots$

D.4 Mechanical testing results of the force vs elongation with the PAM inflated to $48 \mathrm{kPa}$ and with a vertical displacement of $8 \mathrm{~cm} \ldots \ldots$. . 
D.5 Mechanical testing results of the ideal spring force vs elongation with the PAM inflated to $70 \mathrm{kPa}$ and with a vertical displacement of $6 \mathrm{~cm}$. The percentage of the ideal spring force was fitted to match the PAM force. The ideal spring force in this case is set to $35 \% \ldots \ldots \ldots \ldots$

D.6 Mechanical testing results of the ideal spring force vs elongation with the PAM inflated to $55 \mathrm{kPa}$ and with a vertical displacement of $7 \mathrm{~cm}$. The percentage of the ideal spring force was fitted to match the PAM force. The ideal spring force in this case is set to $40 \%$. . . . . . . .

D.7 Mechanical testing results of the ideal spring force vs elongation with the PAM inflated to $48 \mathrm{kPa}$ and with a vertical displacement of $8 \mathrm{~cm}$. The percentage of the ideal spring force was fitted to match the PAM force. The ideal spring force in this case is set to $50 \% \ldots \ldots \ldots \ldots$. . .

D.8 Mechanical testing results of the torque vs percentage of gait cycle with the PAM inflated to $70 \mathrm{kPa}$ and with a vertical displacement of $5 \mathrm{~cm}$. .

D.9 Mechanical testing results of the torque vs percentage of gait cycle with the PAM inflated to $70 \mathrm{kPa}$ and with a vertical displacement of $6 \mathrm{~cm}$. .

D.10 Mechanical testing results of the torque vs percentage of gait cycle with the PAM inflated to $55 \mathrm{kPa}$ and with a vertical displacement of $7 \mathrm{~cm}$. .

D.11 Mechanical testing results of the torque vs percentage of gait cycle with the PAM inflated to $48 \mathrm{kPa}$ and with a vertical displacement of $8 \mathrm{~cm}$. .

D.12 Mechanical testing results of the torque vs percentage of gait cycle with the PAM inflated to $70 \mathrm{kPa}$ and with a vertical displacement of $6 \mathrm{~cm}$. The percentage of the biological ankle torque was fitted to match the PAM torque. The biological ankle torque in this case is set to $35 \% \ldots$ 168

D.13 Mechanical testing results of the torque vs percentage of gait cycle with the PAM inflated to $55 \mathrm{kPa}$ and with a vertical displacement of $7 \mathrm{~cm}$. The percentage of the ideal spring force was fitted to match the PAM force. The ideal spring force in this case is set to $40 \%$. . . . . . . .

D.14 Mechanical testing results of the torque vs percentage of gait cycle with the PAM inflated to $48 \mathrm{kPa}$ and with a vertical displacement of $8 \mathrm{~cm}$. The percentage of the ideal spring force was fitted to match the PAM force. The ideal spring force in this case is set to $50 \% \ldots \ldots$. . . . . . 170

E.1 Walking trial ankle angle for exoskeleton with PAM inflated to $70 \mathrm{kPa} \quad 171$

E.2 Walking trial knee angle for exoskeleton with PAM inflated to $70 \mathrm{kPa}$. 172

E.3 Walking trial hip angle for exoskeleton with PAM inflated to $70 \mathrm{kPa}$. . 173

E.4 Walking trial spring length for exoskeleton with PAM inflated to $70 \mathrm{kPa} 173$ 
E.5 Walking trial ankle angle for exoskeleton with PAM inflated to $40 \mathrm{kPa} \quad 174$

E.6 Walking trial knee angle for exoskeleton with PAM inflated to $40 \mathrm{kPa}$. 175

E.7 Walking trial hip angle for exoskeleton with PAM inflated to $40 \mathrm{kPa}$. . 176

E.8 Walking trial spring length for exoskeleton with PAM inflated to $40 \mathrm{kPa} 176$

E.9 Walking trial ankle angle for exoskeleton with rubber band used as spring element . . . . . . . . . . . . . . . . . . 177

E.10 Walking trial knee angle for exoskeleton with rubber band used as spring element . . . . . . . . . . . . . . . . 178

E.11 Walking trial hip angle for exoskeleton with rubber band used as spring element . . . . . . . . . . . . . . . . . . . . . . . . . . 179

E.12 Walking trial spring length for exoskeleton with rubber band used as spring element . . . . . . . . . . . . . . . . . . . . . . . 179

E.13 Walking trial ankle angle for exoskeleton with no spring element . . . 180

E.14 Walking trial knee angle for exoskeleton with no spring element . . . . 181

E.15 Walking trial hip angle for exoskeleton with no spring element . . . . . 182

E.16 Walking trial spring length for exoskeleton no spring element. In this case it is the length between the anchor points . . . . . . . . . . 182

E.17 Ankle angle for baseline walking trial (without exoskeleton) . . . . . 183

E.18 Knee angle for baseline walking trial (without exoskeleton) . . . . . . . 184

E.19 Hip angle for baseline walking trial (without exoskeleton) . . . . . . 185

E.20 EMG output of the walking trial without exoskeleton. For participant 1186

E.21 EMG output of the walking trial with the exoskeleton but with no spring element. For participant $1 \ldots \ldots \ldots$. . . . . . 186

E.22 EMG output of the walking trial with the exoskeleton with an elastic band as the spring element. For participant $1 \ldots \ldots$. . . . . . 187

E.23 EMG output of the walking trial with the exoskeleton with PAM inflated to $40 \mathrm{kPa}$ as the spring element. For participant $1 \ldots \ldots$. . . 187

E.24 EMG output of the walking trial with the exoskeleton with PAM inflated to $70 \mathrm{kPa}$ as the spring element. For participant $1 \ldots \ldots$. . . 188

E.25 Comparison of the muscle activation in the Right Tibialis Anterior during the PAM $70 \mathrm{kPa}$, PAM $40 \mathrm{kPa}$, Elastic, and baseline trials for participant 1 . The exoskeleton was worn on the Right leg . . . . . . . . . 188

E.26 Comparison of the muscle activation in the Right Gastrocnemius Medialis during the PAM $70 \mathrm{kPa}$, PAM $40 \mathrm{kPa}$, Elastic, and baseline trials for participant 1. The exoskeleton was worn on the Right leg $\quad \ldots . .189$ 
E.27 Comparison of the muscle activation in the Right Soleus during the PAM $70 \mathrm{kPa}$, PAM $40 \mathrm{kPa}$, Elastic, and baseline trials for participant 1 . The exoskeleton was worn on the Right leg . . . . . . . . . . . . 190

E.28 Comparison of the muscle activation in the Left Tibialis Anterior during the PAM $70 \mathrm{kPa}$, PAM $40 \mathrm{kPa}$, Elastic, and baseline trials for participant 1. The exoskeleton was worn on the Right leg . . . . . . . . . . 191

E.29 Comparison of the muscle activation in the Left Gastrocnemius Medialis during the PAM $70 \mathrm{kPa}$, PAM $40 \mathrm{kPa}$, Elastic, and baseline trials for participant 1. The exoskeleton was worn on the Right leg . . . . . . . . 192

E.30 Comparison of the muscle activation in the Left Soleus during the PAM $70 \mathrm{kPa}, \mathrm{PAM} 40 \mathrm{kPa}$, Elastic, and baseline trials for participant 1 . The exoskeleton was worn on the Right leg . . . . . . . . . . . . . . 193

G.1 Image of the Simulink MatLab simulation block diagram . . . . . . 197 


\section{List of Tables}

3.1 Geometric properties of PAM and theoretical strain of elastic bladder based on braid angle equations. . . . . . . . . . . . . . 30

3.2 Geometric properties of PAM and experimental strain of elastic bladder obtained using physical measurements. . . . . . . . . . . . . 31

3.3 Parameters for biaxial bladder elongation tests . . . . . . . . . . 33

3.4 Geometric measurements of test muscles for high and low pressure

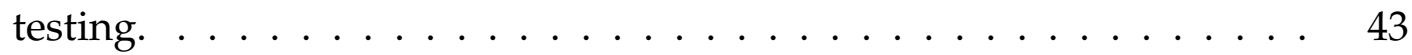

3.5 Elongation of test muscle during cyclical test . . . . . . . . . . . 44

3.6 Temperature fluctuation of gas inside PAM . . . . . . . . . . . 54

4.1 Summary of design restrictions and other desirable criteria . . . . . . 58

4.2 Physical parameters and values related to the mechanical testing of the prototype exoskeleton. The muscle length refers to the distance between the attachment points, including the end caps . . . . . . . . 74

5.1 Average variation in joint range of motion during walking trials for both legs (with and without exoskeleton) _. . . . . . . . . . 96

5.2 Average walking speed during human testing for participants 1-5. Speeds presented in $\mathrm{m} / \mathrm{s} \ldots \ldots \ldots \ldots . \ldots \ldots$

5.3 Average muscle activation values when compared to baseline for both legs (with and without exoskeleton) . . . . . . . . . . 102

A.1 Table summarizing the typical Range of Motion of the lower limb joints

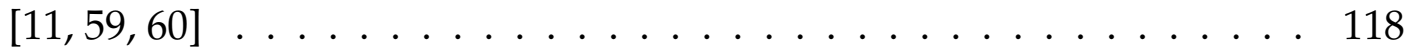

A.2 Lower limb muscles and their functions related to movements [61, 60, 11]119

C.1 Muscle characteristics for PAM model validation tests for a n2 PAM inflated to $138 \mathrm{kPa}$ and tested at $80 \% 90 \%$ and $95 \%$ of its maximum

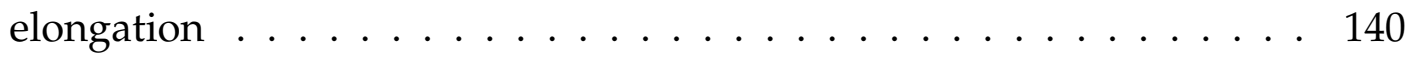


C.2 Muscle characteristics for PAM model validation tests for a n2 PAM inflated to $207 \mathrm{kPa}$ and tested at $80 \% 90 \%$ and $95 \%$ of its maximum elongation ........................ 141

C.3 Muscle characteristics for PAM model validation tests for a n2 PAM inflated to $276 \mathrm{kPa}$ and tested at $80 \% 90 \%$ and $95 \%$ of its maximum elongation . . . . . . . . . . . . . . . . . . . . . . 141

C.4 Table of various PAM variables and their values . . . . . . . . . . . . . 142

C.5 RMSE values for low pressure PAM tests . . . . . . . . . . . . . . 155

C.6 Difference between peak value of experimental results and simulated results for low pressure PAM tests. Negative value indicates that the peak of the experimental results is lower in magnitude than the simulated results . . . . . . . . . . . . . . . . . . . . . . . 156

C.7 RMSE values for high pressure PAM tests with and without the bladder stress model . . . . . . . . . . . . . . . . . . . . . . . . . . . 157

C.8 Difference between peak value of experimental results and simulated results for lhigh pressure PAM tests. Negative value indicates that the peak of the experimental results is lower in magnitude than the simulated results . . . . . . . . . . . . . . . . . . . . . 158 


\section{Chapter 1: Introduction}

The mobility of individuals is vital to their everyday activities and has been directly linked to their quality of life [1]. Unfortunately, physical difficulties arising from aging, trauma or diseases often greatly limit the independence of otherwise healthy people. Moreover, fatigue and instability can make locomotion energy inefficient and difficult over long distances. Nowadays, with our aging population and other health challenges, there is a great need to assist individuals to stay active in order to sustain their quality of life and also mitigate the effects that a sedentary lifestyle could have on their health and overall well-being.

Walking assist exoskeletons are wearable mechanical devices that assist or enhance human locomotion. These devices have proven to be very effective at reducing the human effort required for ambulation. Despite this, all present robotic exoskeletons cost tens of thousands of dollars [2] and are thus only available to specialized research or rehabilitation facilities. Furthermore, these devices are limited to only a few hours of operation due their massive power demands [2,3]. A large part of this energy goes towards offsetting the weight of the exoskeleton or running sensors and control systems. For these reasons, under actuated and passive (non powered) exoskeletons are becoming increasingly popular.

Passive walking assist exoskeletons are devices that attempt to draw energy out of the lower limbs so that it can be recycled to assist a specific movement. A number of different strategies have been applied to the development of passive walking assist devices to decrease the energy expenditure of the wearer. Some designs focus on nearly imperceptible body weight support systems [4], while some use complex spring systems in an attempt to save energy by reducing muscular requirements [5]. The human body is already very efficient, it has proven to be a challenge to synergistically improve its efficiency with exoskeleton devices.

During the gait cycle, the hips and knees produce a complex series of torques 
to stabilize the body and ensure a smooth transition from one supporting leg to the other. The ankle however, serves mainly a single purpose. It takes advantage of the elastic nature of the Achilles tendon to produce a large torque during the push off phase of the gait. This torque propels the body forward into the next step. This rapid release of energy corresponds to over half of the total energy generated during walking $[6,7]$. The passive elongation and recoil of the Achilles tendon has historically been emulated in an attempt to reduce the requirements of the biological system. Generally, these devices have been unable to reduce the energetic cost of walking for healthy individuals. However, recently, one passive ankle exoskeleton has been able to show a reduction in the metabolic cost of walking [8]. It achieved this by mimicking the passive stretch and recoil of the Achilles tendon. The main drawback of this design is its limited adaptability to different users or walking speeds. The device uses a timing pin system that needs to be physically adjusted to allow the user to modify their walking pace. Furthermore, the Achilles is simulated using a simple coil spring. This does not truly capture the non-linear stiffness of the biological tissues and thus, does not produce the most realistic ankle torque profile. A device that addresses these issues could improve the current state of walking assist exoskeletons.

A Pneumatic Artificial Muscle (PAM) is a flexible uniaxial actuator. It is made of an airtight inner bladder surrounded by a woven mesh. The two layers are clamped tightly at both ends of the muscle forming a seal. When the internal bladder is inflated, the diameter of the PAM increases, due to the fixed length of the fibers in the mesh. This inflation also generates a contraction along the longitudinal axis. These actuators have been used as actuators in active exoskeleton development by multiple researchers and are favored because of their light weight, flexibility and for their non linear stiffness. $[9,10,11]$

(a)

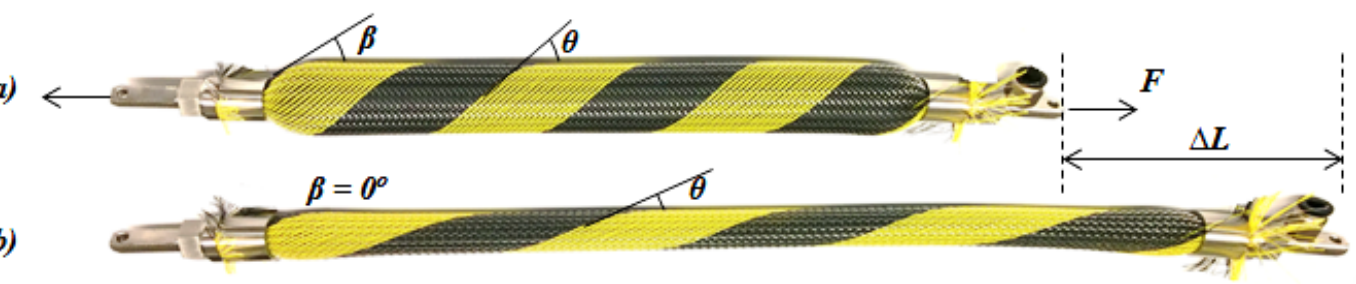

Figure 1.1: PAM prototype a) Pressurized state b) Deflated state

While past models have characterized the PAM either in a static force elongation or dynamic contraction scenarios, little if any work has evaluated the PAM in a 
passive elongation setting. This work will use the PAM as a passive actuator in the design of a walking assist ankle exoskeleton.

\subsection{Objectives}

The objective of this work is to design a passive ankle exoskeleton that incorporates a PAM as a passive non-linear elastic element mounted parallel to the calf muscle and Achilles tendon such to reduce muscular effort of healthy users during walking.

\subsection{Methodology}

The first section of this work relates to the analytical modelling of PAM. An established model was used as a reference [12] and, two shortcomings of the model were addressed. Improvements were proposed and tested in an attempt to improve the accuracy of the existing model. First, the mechanical properties of the elastomer bladder material were re-evaluated. Second the isothermal gas assumption was replaced by a polytropic assumption. In order to evaluate the validity of these two changes, experimental testing was carried out and compared to a model simulation.

The second part of this work was the design, fabrication and testing of a passive ankle exoskeleton. A 2D simulation was created in order to predict the behaviour of a physical exoskeleton prototype prior to construction. Once the device was fabricated, purely mechanical testing was achieved to examine the timing mechanism performance and torque generation throughout the full range of motion. Human walking trials were then carried out to examine the device's overall performance and validate the ability of the device to reduce the effort of the calf muscles during walking.

\subsection{Contributions}

- A comprehensive literature review was conducted in order to establish the current state of walking assist devices and identify their drawbacks. Furthermore, 
various assistive strategies were evaluated to guide the design strategy of this research.

- While many static and dynamic models have been proposed and validated in open literature, none consider and predict the PAM as a passive elastic element undergoing cycle loading. This work adapted an existing PAM dynamic model and further developed it to predict PAM behaviour under distinct operation. Among other enhancements, two limitations of the present model were re-evaluated, namely biaxial stress in the bladder as well as a polytropic gas assumption. A computer simulation compared with experimental testing evaluated the newly proposed PAM model.

- Recent research by Wiggin, Sawicki and Collins [13], has showed the ability of a passive ankle exoskeleton to reduce the metabolic cost of walking. While this is a remarkable achievement, further improvement was sought in the current design. For this research. A PAM based passive ankle exoskeleton incorporating a novel timing mechanism is proposed. Distinctively, the elastic behaviour of the PAM closely emulates the mechanical behaviour of the skeletal muscle tendon unit and the proposed timing mechanism offers an adjustable feature to customize the engagement and disengagement of the device for different users or walking speeds. The proposed design was fabricated and mechanically tested in order to evaluate the timing mechanism and the device's ability to produce sufficient torques. Figure 1.2 presents the final prototype.

- Human testing was carried out to evaluate the overall performance of the ankle exoskeleton as well its ability to reduce the muscular effort of walking. Results found that the angular range of motion was reduced by the exoskeleton and highlighted specific flaws in the testing methodology as well as the mechanical design. 


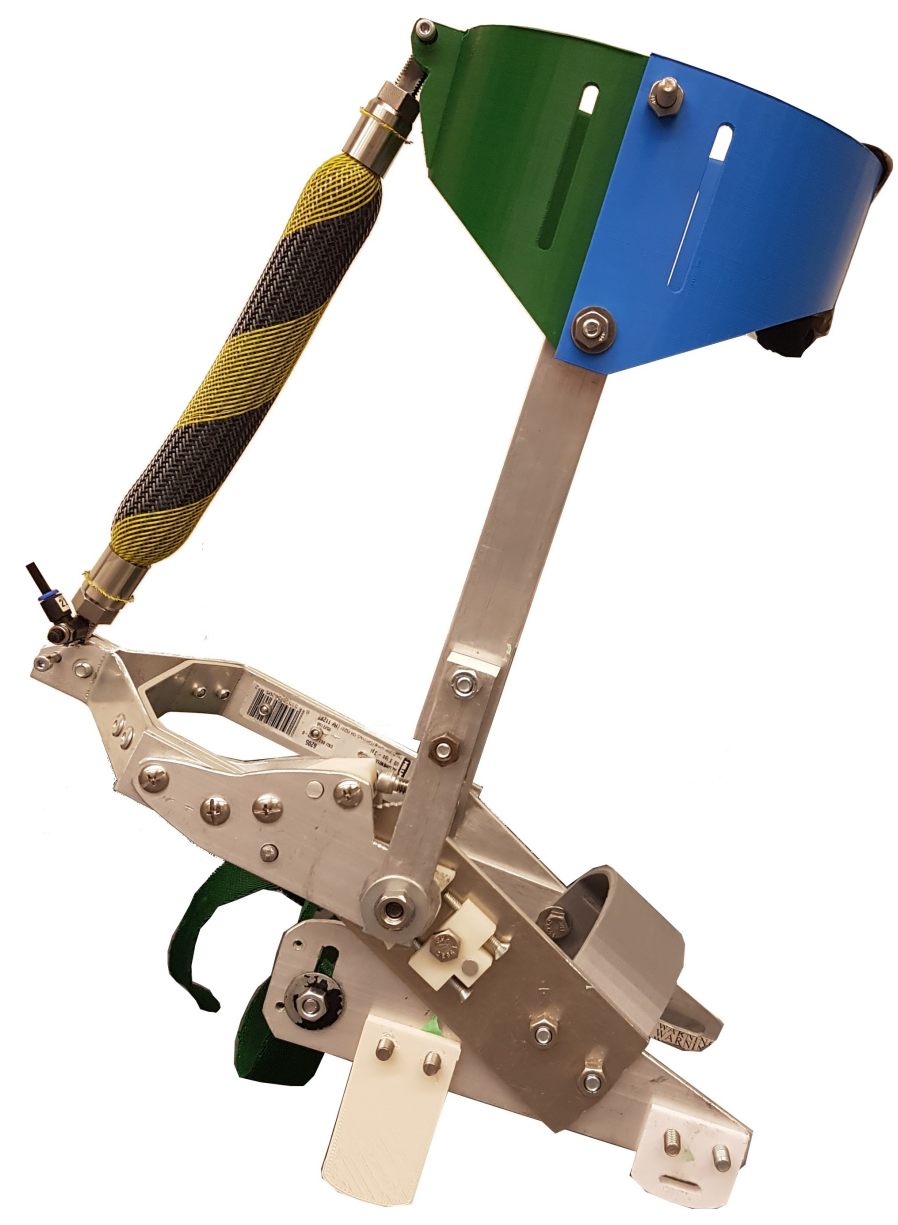

Figure 1.2: Photograph of the final prototype

\subsection{Thesis Outline}

This thesis is divided into seven chapters. Chapter 1 introduces the general information regarding the problem that will be addressed as well as the approaches utilized. The objective of this research and the achieved contributions are also included.

Chapter 2 contains a review of the human gait cycle, previous devices as well as an evaluation of their effectiveness. The PAM is also introduced. Current analytical models describing the mechanical behaviour of the PAM are also presented and critiqued.

Chapter 3 describes the analytical modelling of the PAM. This includes the development of the proposed model as well as a the validation of the model through experimental testing and simulation. 
Chapter 4 presents the design, modelling, and fabrication of the proposed device. The requirements of the device are presented and used to guide the design. The function and construction of the timing mechanism is presented. This chapter also describes the mechanical testing of the exoskeleton device. The results are presented and compared to normal human walking data.

Chapter 5 describes the human testing procedures and results. The testing utilized motion capture and EMG to evaluate the function of the device. Four different device configurations were tested and compared to normal walking in order to assess the contribution of the device.

Chapter 6 concludes this work and addresses certain limitations associated with the device itself as well as the testing and methodology. Furthermore, suggestions for future work and improvements are suggested. 


\section{Chapter 2: Literature Review}

The literature review chapter begins by presenting various exoskeleton devices on the market and in research laboratories, subsequently, their advantages and disadvantages are presented and discussed.

In the second part of this chapter, the PAM is presented and discussed with emphasis on analytical models found in open literature.

The basic anatomy of the lower limbs and the principles of human walking, as they relate to exoskeleton design, are found in Appendix A. 


\subsection{Walking Assist Devices}

\subsubsection{Assistive motion}

The ankle is a complex joint and has three axes of rotation each with a different range of motion. The largest movements are plantar and dorsiflexion in the sagittal plane. Due to the limited movement in the frontal and transverse planes, walking analyses are often done in the sagittal plane only. To examine the contribution of joints of the lower limbs in three dimensions David et al. [14], examined 11 male participants at four different locomotion speeds. A sample of the results can be seen in Figure 2.1

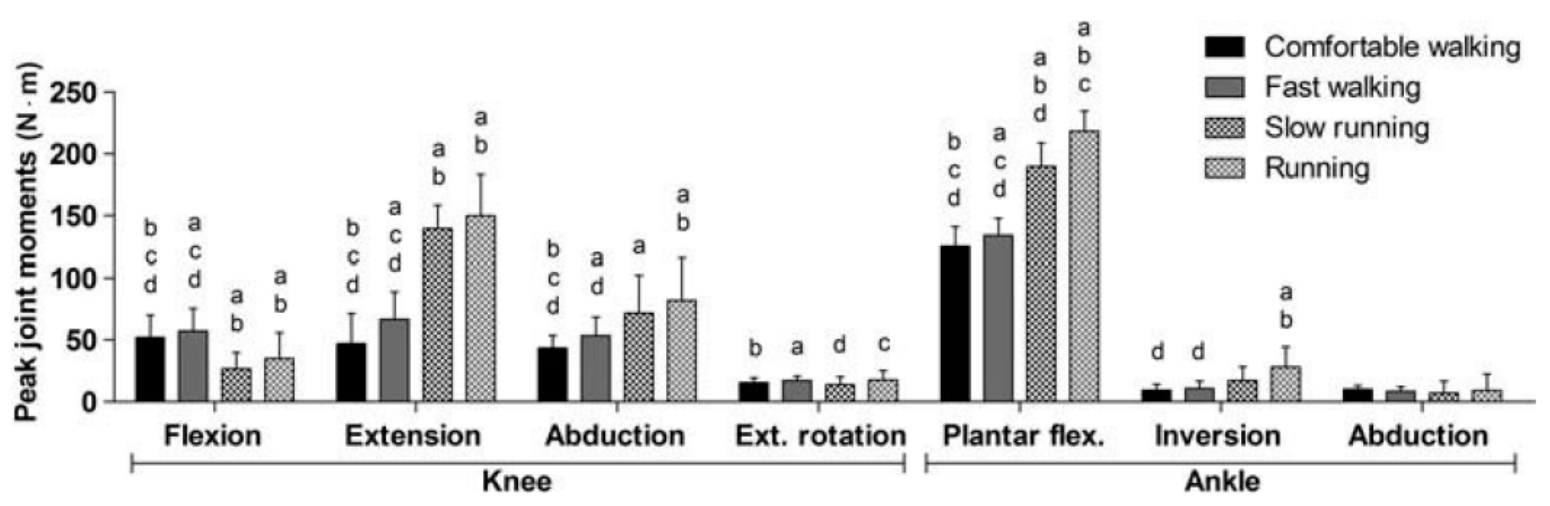

Figure 2.1: Peak values for joint moments at the knee and ankle joints. Data are presented as mean (bars) and standard deviation (vertical lines) for the group $(n=11)$. a-significantly different from comfortable walking $(\mathrm{P}<0.05)$; b-significantly different from fast walking $(\mathrm{P}<0.05)$; $\mathrm{c}$-significantly different from slow running $(\mathrm{P}<0.05)$; $\mathrm{d}$-significantly different from running $(\mathrm{P}<0.05)$. Image and caption taken from [14]

The results illustrate that regardless of the speed of locomotion, plantar and dorsiflexion moments of the ankle are much larger than moments in the other planes. This demonstrates that for the ankle joint during walking, the majority of the angular movement as well as the majority of the torque generation occurs in the sagittal plane. Therefore most researchers developing walking assist devices confine their designs to the sagittal plane. 


\subsubsection{Assistive devices}

Examinations of the human gait cycle, such as Figure A.14 in Appendix A,clearly demonstrate areas of large power generation inside the human body. Consequently, it has been suggested that reducing the power that the body must generate should directly reduce the effort of the individual.

Honda, the well-known automotive manufacturer, has developed what they call the Honda Stride assist, Figure 2.2. Small and lightweight, this device was created for individuals who are able to walk unaided but who would benefit from some form of walking assist. The device is worn like a belt and features two bars that extend down the leg and attach to a strap around the thigh. Angular position sensors and an on-board microprocessor control the actuation of the electric motors. The device provides assistance in the form of a hip flexion moment. In experimental testing the device was shown to increase the walking speed of elderly individuals as well as decrease the energy consumption of young adults during locomotion [15, 16]. The most significant drawback of the device is that, like similar devices, the battery life is very limited. The Honda device can sustain approximately 60 minutes of continuous use [3].

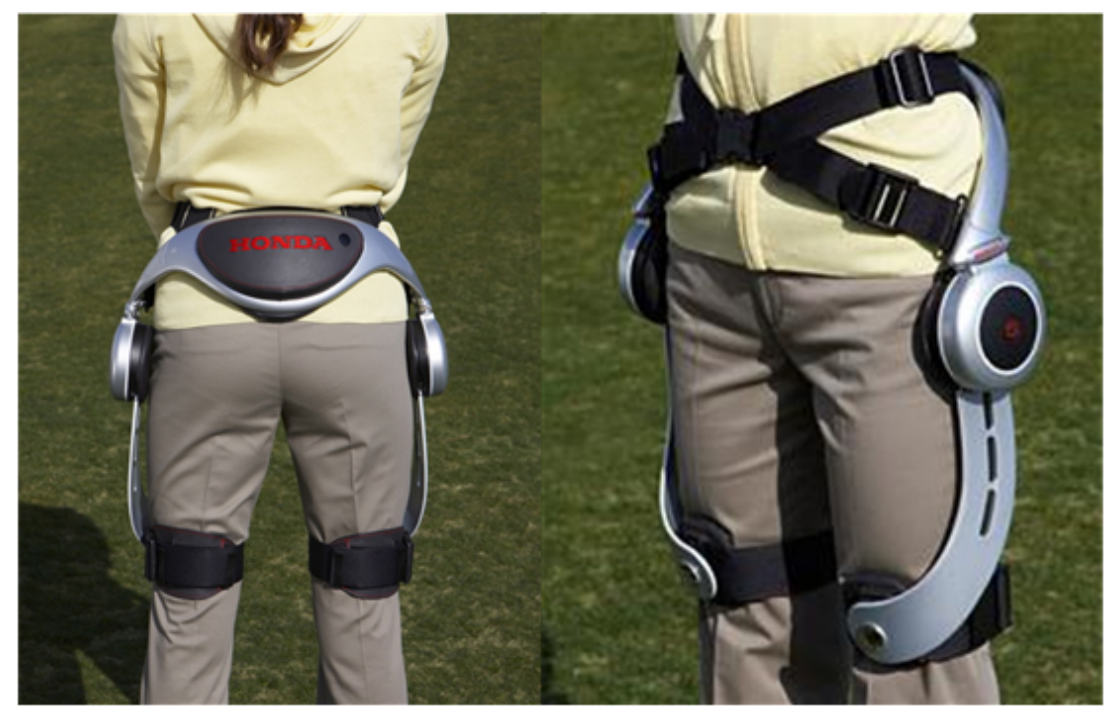

Figure 2.2: The Honda Walking Assist Device with Stride Management System [17]

Devices such as the Honda stride assist demonstrate that mechanical devices can reduce the energetic requirements of the human body. This principle is taken to the limit when considering devices made for paraplegic persons. Not only must the 
device assist the user, but in this case, it is solely responsible for the movement of the lower limbs. One such device is the ReWalk, as seen in Figure 2.3.

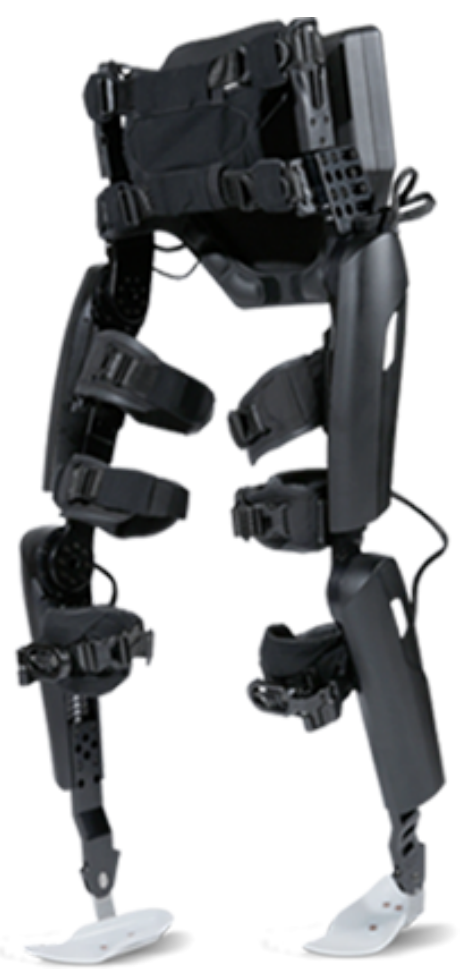

Figure 2.3: ReWalk 6 exoskeleton suit by ReWalk Robotics [18]

The ReWalk can be used continuously for up to four hours. Currently the device has a price tag of \$ 69500 to \$ 85000 USD [2]. For this reason, computer controlled walking assist devices are simply beyond the reach of the average person and cannot currently benefit significant portions of the population.

The two devices introduced in this section represent a much larger pool of robotic and computer controlled walking assist exoskeletons. The main challenges of these devices are the large weight, short battery life and the high price point. Therefore simpler designs and single joint mechanisms are attractive low cost alternatives.

\section{Ankle Exoskeletons}

Walking involves three main articulations namely the hip, knee and ankle. Figures A.14 in SectionA.1.5 of Appendix refAppendix demonstrates the relative contributions of these joints. Specifically Figure A.14 C), presents the power produced at each joint during walking. The graph illustrates the complex sequence of positive and 
negative powers that are generated at the hip and knee. By contrast, the power generation in the ankle is essentially confined to a single area of negative power followed by an area of positive power. This simple behaviour during walking, and the nearly equal positive and negative powers, make the ankle joint a promising target for gait assist devices.

Multiple studies have been done to examine the effect of augmented ankle torque. Norris et al. [9] used a pneumatic ankle exoskeleton, shown in Figure 2.4, to evaluate the ankle push off power as well as the metabolic cost of walking in nine young and seven elderly individuals.

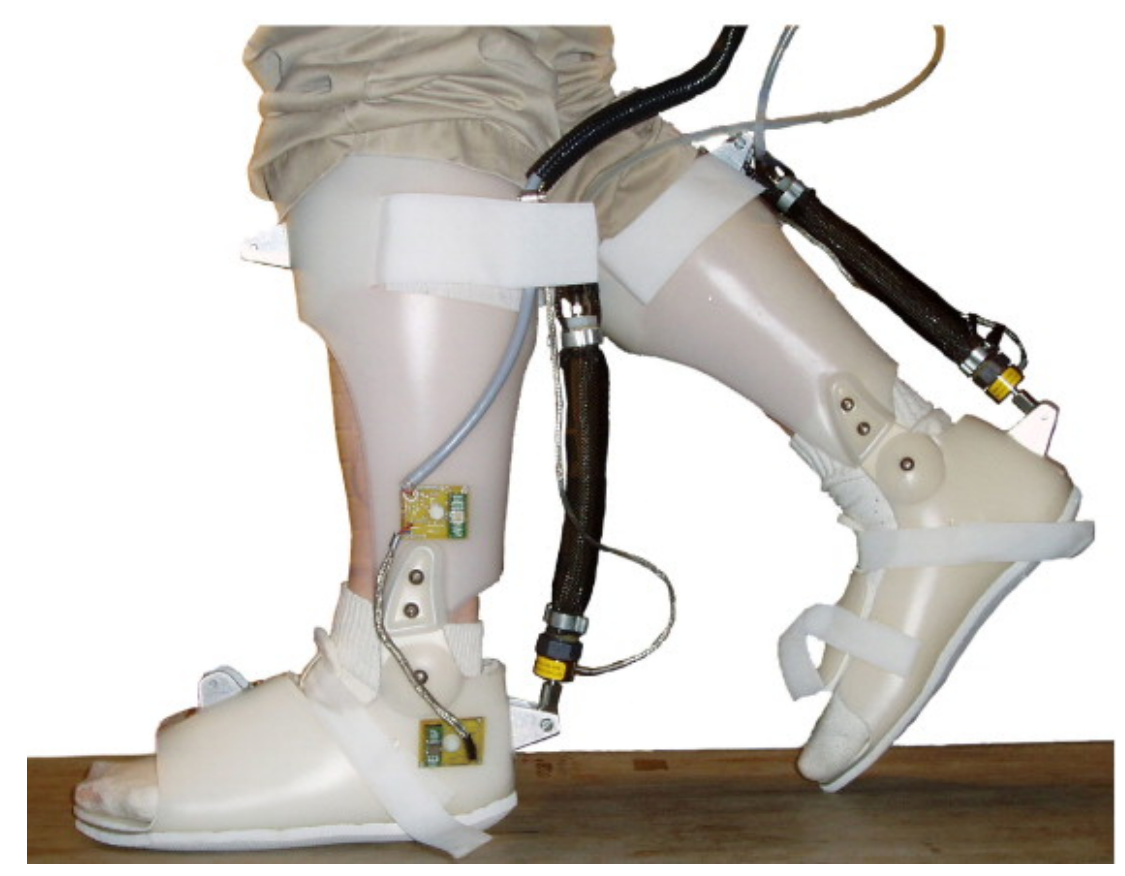

Figure 2.4: Photograph of the ankle exoskeleton prototype [9]

The researchers found that younger individuals showed increased preferred walking speed, increased total (Biological and exoskeleton) ankle torque, as well as a decrease in the metabolic cost of transport. Three out of seven elderly individuals followed the same trends as the young adults. The authors hypothesized that the younger subjects were able to adapt to the device more rapidly and that over a longer time frame the elderly individuals would present faster walking speeds and lower metabolic cost. Sawicki et al. [19] also demonstrated decreases in metabolic cost while walking with a tethered ankle exoskeleton. They reported energy savings of $10 \%$ when walking on a level treadmill. The results of this device demonstrate the effectiveness of an ankle exoskeleton; however, the fact that the device was tethered 
to a power source and control unit confine its benefits to the laboratory setting.

\subsubsection{Hybrid - Minimally Powered Devices}

Human augmentation devices and powered exoskeletons are generally very limited by their power supplies. Some classes of exoskeletons cannot avoid this problem. Robotic suits intended for military use or for strength augmentation inevitably require large amounts of power. However devices that aim to assist walking do not necessarily require such large power sources. This section presents devices that do not rely entirely on external power sources. Instead these hybrid devices use a combination of external power and energy already contained in the gait cycle. These devices all attempt to re-create natural human motion and/or reduce the metabolic cost of walking.

\section{Untethered Ankle Devices}

In 2010 Collins et al. [20] developed a microprocessor controlled prosthetic foot whose goal is to capture energy that is usually lost during heel strike and to release it in the form of positive work, Figure 2.5. The design uses a series of springs in order to produce a plantar flexion torque during terminal stance. Healthy subjects tested the device by wearing an amputation simulator device. This allowed subjects to don the prosthetic during testing as well as serve as their own baseline. Conventional prostheses were shown to increase metabolic cost by $23 \%$ [20]. The prototype design successfully generated the desired plantarflexion moment and reduced the metabolic toll of walking to $14 \%$. Therefore, the device reduced the effort required to walk with a prosthetic foot, however it is still higher than healthy walking. 

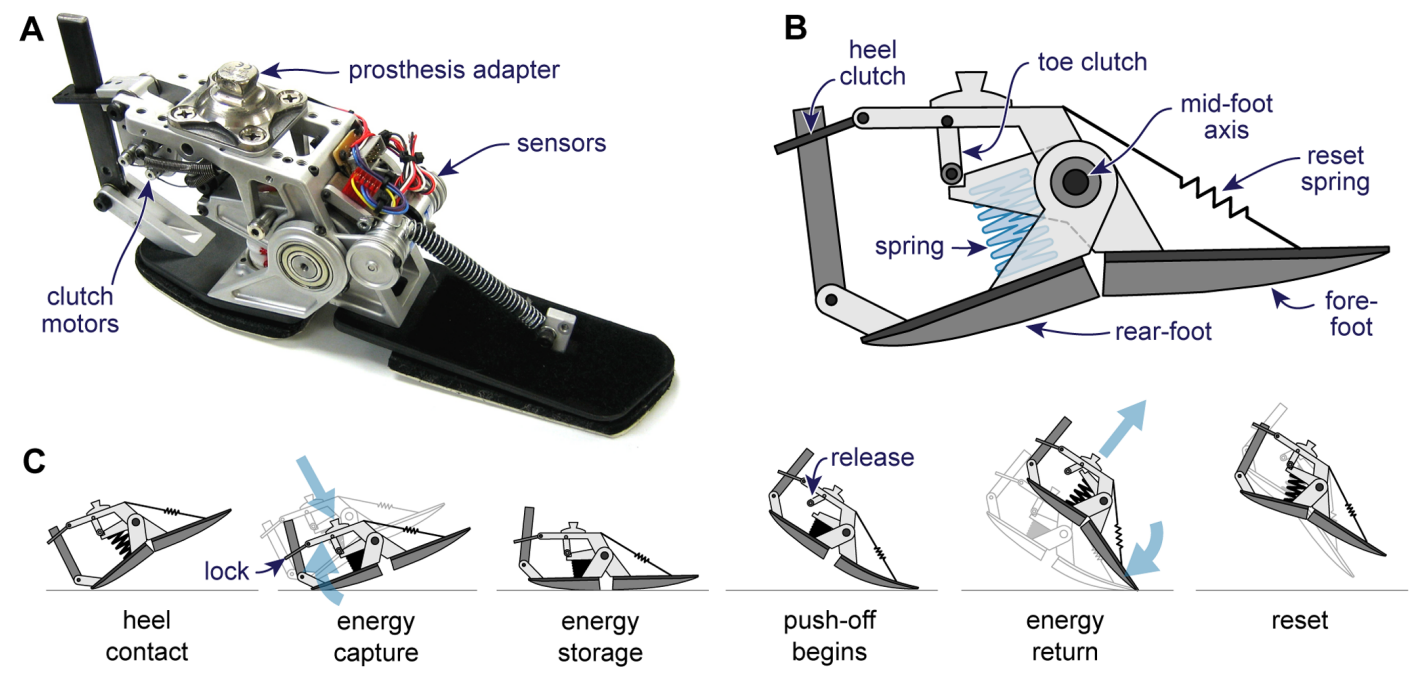

Figure 2.5: A) Prototype energy recycling device B) the main mechanical components of the device $\mathrm{C}$ ) Position and action of the device during walking. The heel spring is compressed during the energy capture and energy storage phase. The energy return phase corresponds to the release of the stored energy in the form of a plantar flexion moment. [20]

The results demonstrate the role that the plantarflexion moment, present during terminal stance, has on gait efficiency. Furthermore, it accomplished this without using work produced by external energy sources. The actuation force is taken from the gait cycle, external energy is used only for timing and control. A subsequent study, [21], further examined the performance of the device. The results indicated that the energy recycling foot was able to produce higher push off work as well as reduce the impact work during heel strike and weight acceptance when compared to conventional prostheses. Despite this, walking with the device was more metabolically costly than walking with the subjects prescribed prosthesis. The authors postulate that the increase in metabolic cost may be due to the weight of the device, or the insufficient acclimatization period. The researchers allowed for one training session at least two days prior to testing and one longer subsequent training session. While these training sessions likely helped reduce the changes in kinematics associated with new walking devices, the time required to fully adapt to a prosthetic foot has not been fully explored [22].

Another energy storing prosthetic foot is the AMP 2.0 [23], shown in Figure 2.6, this device stores energy during the entire stance phase which is then released during terminal stance. This design takes advantage of the captured energy to reduce the requirements of electrical actuation. The design uses a plantar flexion spring and a 
push off spring separately. The push off spring stores energy but does not generate any ankle moment until the locking mechanism is disengaged. The locking mechanism can be seen in Figure 2.7. It consists of a four bar linkage that is allowed to pass into a jamming position. In order to unlock the mechanism a servo motor is used. A single subject walking trial demonstrated the satisfactory working principles of the device [24]. The authors state that future testing will involve an examination of the metabolic cost of walking in clinical trials.

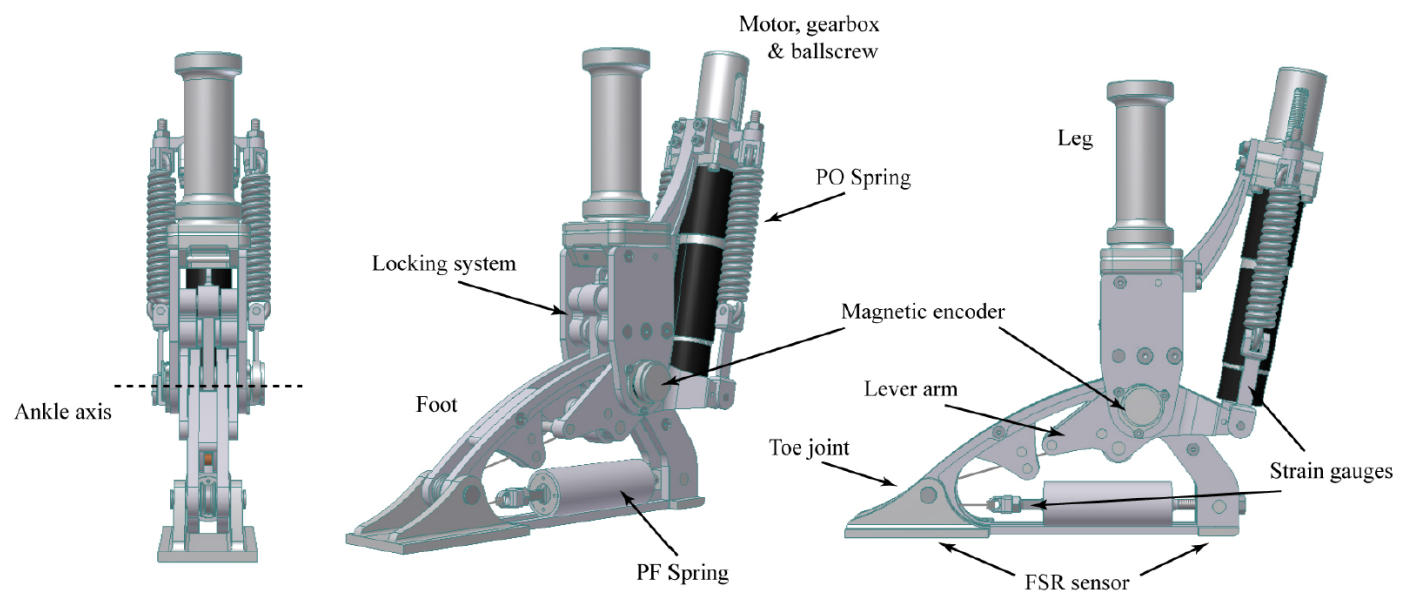

Figure 2.6: The AMP foot 2.0 [23]

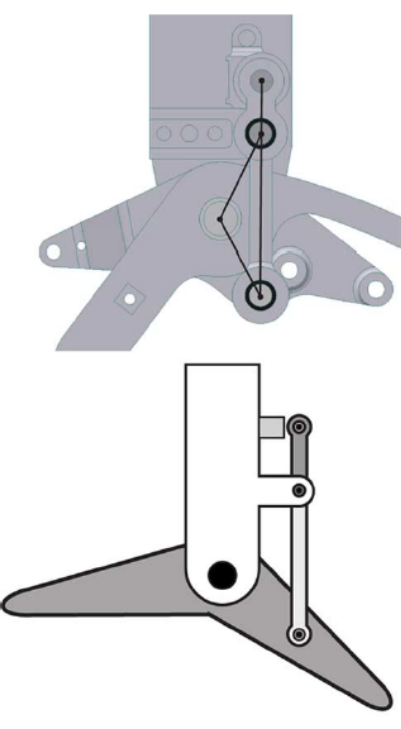

(a)

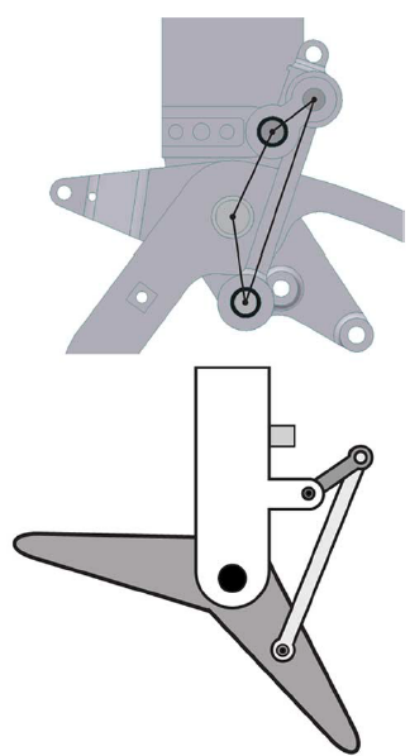

(b)

Figure 2.7: Illustration of the geometric locking mechanism of the AMP 2.0 a) locked position b) unlocked [24] 
The AMP 2.0 uses a locking mechanism to allow the plantarflexion spring to function separately from the push off spring. The plantar flexion spring gathers energy during the controlled dorsiflexion phase of stance. Meanwhile the electric actuator is loading the push off spring. The gradual loading of the push off spring can be compared to the series elastic loading of the human ankle. Various studies [25], [26] have shown that the human body uses the Achilles tendon to store energy during controlled dorsiflexion. In high speed activities such as running the Achilles tendon also helps reduce the required contraction speed of the Gastrocnemius muscles. By doing so, the biological muscle is able to contract more slowly and more efficiently. The AMP foot's gradual loading of the push off spring can be seen as a similar approach.

Another minimally powered foot prosthesis has been designed by Mooney et al. [27]. The device uses a pneumatic piston as well as a bending spring in an attempt to closely mimic the stiffness of the biological ankle. The underpowered design aims to improve upon passive prosthesis stiffness characteristics. The authors point out that commonly used cantilever leaf springs vary in stiffness as the center of pressure moves anteriorly. By contrast, the biological ankle exhibits an increase in stiffness during the progression of the stance phase [28]. Mechanical testing indicates that this prototype demonstrated stiffness profiles much more closely related to biological values, when compared to current energy storing prostheses.
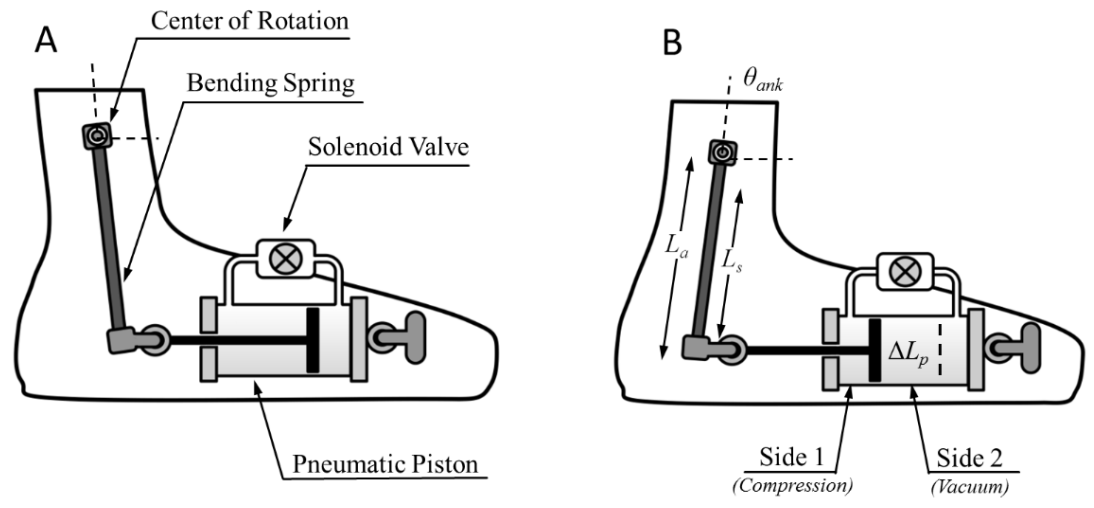

Figure 2.8: Schematic representation of combination pneumatic spring and leaf spring prosthesis [27]

Quasi-passive and underpowered ankle-foot prostheses aim to replicate specific characteristics of the biological system. The energy recycling foot is designed to recover lost heel collision energy in order to assist push off at terminal stance [20]. 
The AMP foot uses a series elastic actuator, similar to the relationship between the Gastrocnemius Medialis and the Achilles tendon, to gradually build up energy [23]. Other researchers [27] aim to assist users by replicating natural ankle stiffness in order to produce more natural gait parameters.

\subsubsection{Passive devices}

This work is intended to design a walking assist device for healthy individuals walking over long distances. For this reason, Ankle Foot Orthoses (AFO), as well as other medical devices prescribed to address a specific gait deficiency, will not be examined in depth. Rather, devices that explore ways to augment the healthy human body without external power supplies will be examined in this section.

\section{Passive Orthoses}

Orthoses are devices that are worn by the user in order to support and assist certain movements and activities. The distinction between orthoses and exoskeletons can be a bit of a gray area. Both are worn in parallel with the body and aim to contribute some level of assistance. However, orthoses are primarily used to restore normal limb function. Exoskeletons on the other hand are generally intended more for augmentation of abilities and performance.

Passive ankle orthoses that store and return energy generally consists of a rigid shell of the lower leg that extends under the foot, see Figure 2.9. Many subtle design variations and different materials are used, however the function remains the same. Essentially the device acts as a leaf spring running parallel to the shank. As the ankle plantar or dorsiflexes the device generates a joint moment that will attempt to return the foot to the neutral position. 


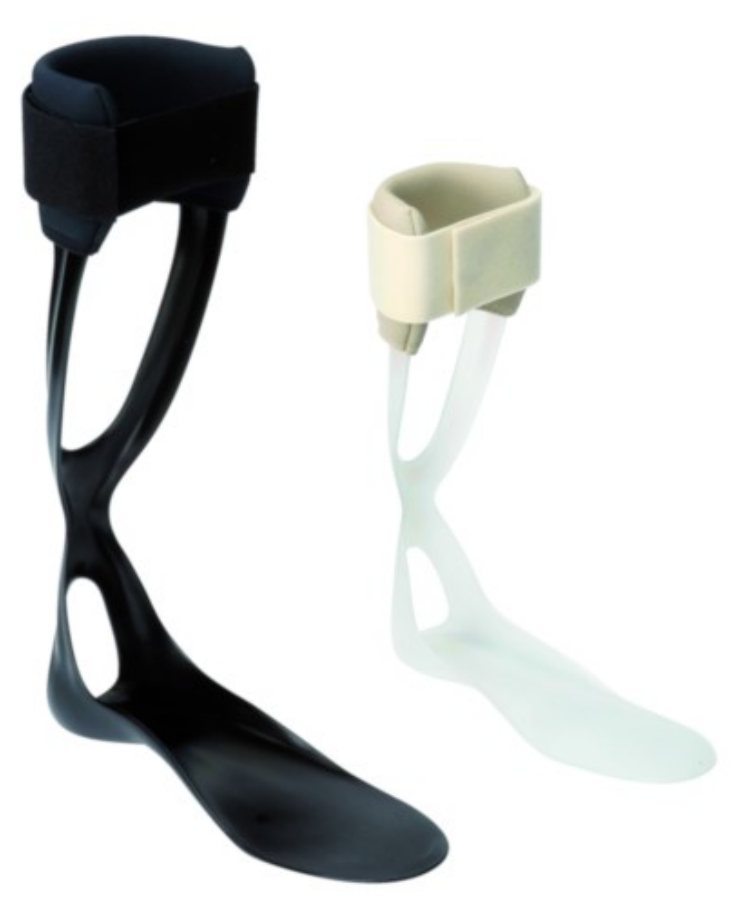

Figure 2.9: Thermoplastic ankle foot orthosis by Ottobock [29]

A number of researchers have examined and confirmed the benefits of deformable orthoses such as these [30],[31]. An individual experiencing difficulty walking is prescribed a specific stiffness of device. The orthosis is then moulded to the person's limb in order to ensure comfortable transfer of forces. This type of device is very beneficial to those with general muscle weakness or other specific issues, however, the simplistic design does not allow for the full range of motion during walking. As such, these device are very helpful in certain cases but overall they do not reduce the effort required to walk.

\section{Passive Exoskeletons}

It has been demonstrated by various researchers that assistance during terminal stance push off can help reduce the metabolic cost of walking [20, 9, 19]. However, doing so with purely passive mechanisms is an ongoing challenge. Especially since the addition of assistive mechanisms inevitably increases the weight of the limb and can increase the metabolic cost of ambulation. In 2015, Kim et al. [32], tested a purely passive mechanical ankle exoskeleton intended to assist vertical jumping. Using three coil springs in parallel with the Achilles tendon, the device was loaded during dorsi- 
flexion and released during plantarflexion, see Figure 2.10.

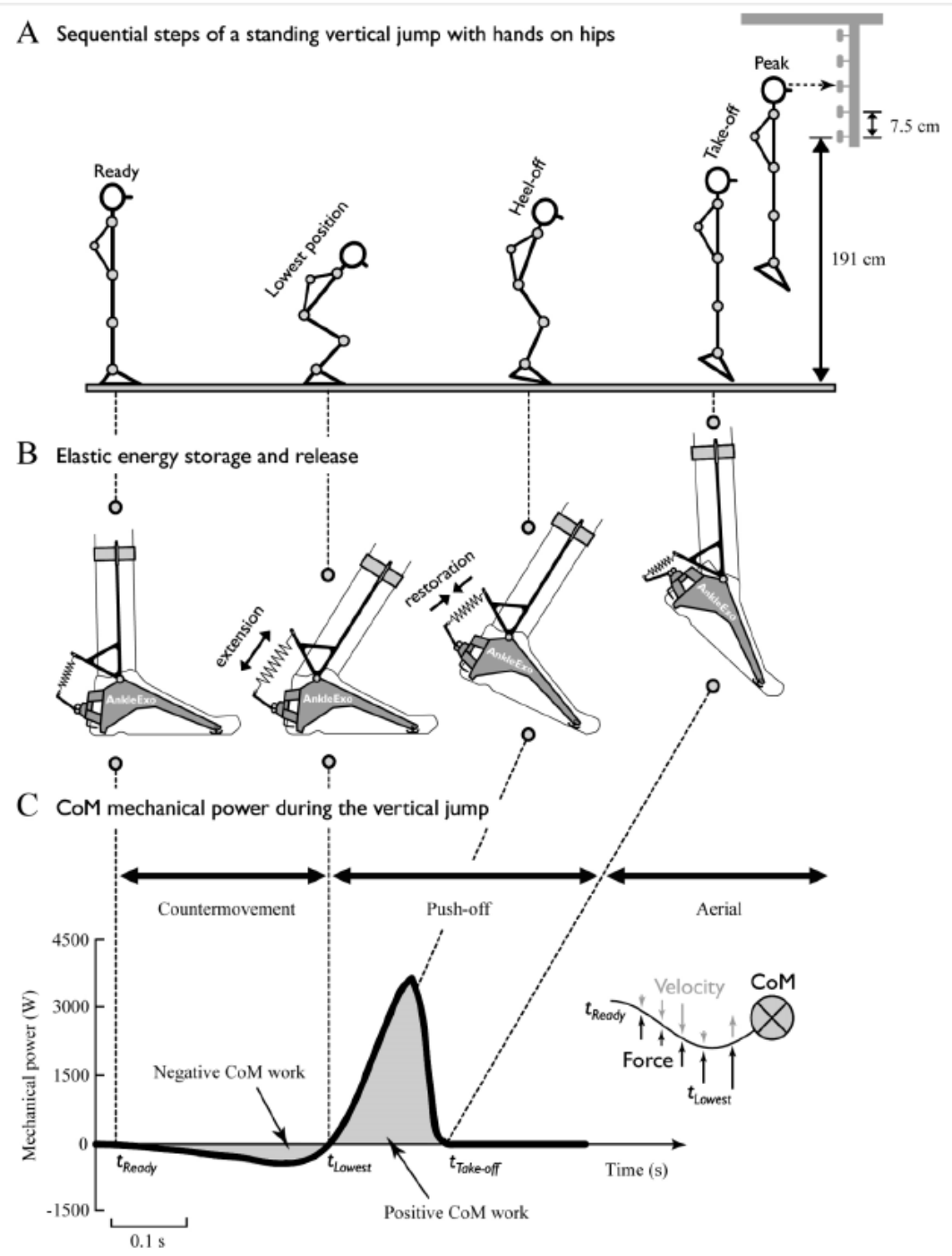

Figure 2.10: A) Simplified steps of a counter movement jump with hands on hips in the sagittal plane. B) Elastic storage and release mechanism of the passive-elastic ankle exoskeleton. C) Center of Mass (CoM) mechanical power during the counter movement jump. CoM work is shown by the shaded area below the curve. Image and description taken from [32]

The results illustrate that the device was light enough to allow for vertical jumping without large changes in joint kinematics. Furthermore, the height of the vertical jump was not increased by the device. This device illustrates that passive devices can capture and restore energy at the ankle joint while still being lightweight enough to allow for normal joint kinematics and ranges of motion. 


\section{Passive Walking Assist Exoskeletons}

There is currently only one ankle exoskeleton that has been shown to reduce the metabolic cost of human walking using purely passive elements [13]. In 2011, a novel "Smart Clutch" was described in a paper by Wiggin, Sawicki and Collins [33]. The authors described a passive exoskeleton than used a standard coil spring positioned in parallel with the Achilles tendon. The major contribution of the paper was the implementation of the so called "Smart Clutch". This device was based on a ratchet type design. During the stance phase the mechanism is locked and store energy in the extension of the coil spring. Once the spring releases this stored energy in the form of a plantar flexion moment, the mechanism is disengaged. This disengagement allowed for the elongation of the entire system with minimal resistance. During the swing phase of gait, the ratchet was allowed to rotate and unwind. Figure 2.11 summarizes the mechanism's function.

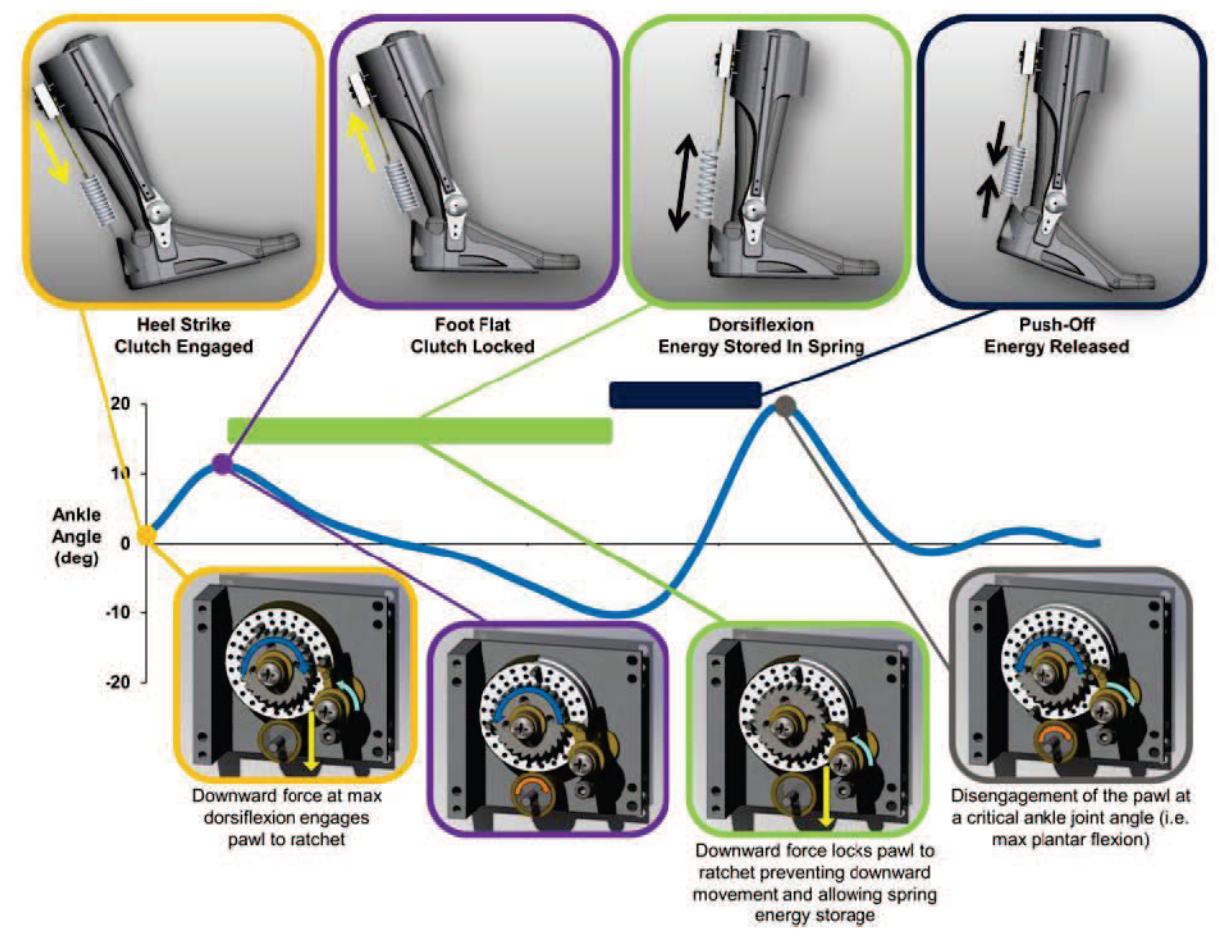

Figure 2.11: Illustration of the implementation of the Smart-Clutch mechanism. Blue line shows the normal ankle joint profile for walking. Image taken from [33]

The authors continued work on this design and in 2015 published the results of physical testing [13]. The study used 9 participants wearing the ankle device. The results indicated a reduction of the metabolic cost of walking of $7.2 \%$. This marks a significant accomplishment in unpowered exoskeleton development. However, the 
design has certain limitations. For instance, the timing of the device is dictated by the positioning of the engagement pins inside the clutch mechanism. Therefore, the device is set for one specific set of walking parameters. To adjust the pins to accommodate a change in walking speed, the internal structure of the mechanism needs to be manually adjusted. This means that the mechanism is set to a single walking speed and is only beneficial within a very narrow window of walking parameters. Furthermore, the device uses a standard spring with a linear stiffness to assist the ankle. However, the stiffness of the ankle is non-linear [34]. Thus the torque profile of the natural joint could theoretically be more closely replicated by a spring with a non-linear stiffness, a PAM for example.

\subsection{The Pneumatic Artificial Muscle}

The PAM is a pneumatically powered unidirectional actuator whose structure and functionality differs significantly from traditional pneumatic actuators (i.e. cylinders). In its most common design, the PAM is made of an elastic bladder that is enclosed in a double helically braided sleeve. Both bladder and sleeve are held airtight at their ends using mechanical fixtures. Figure 2.12 shows an example of the PAM prototype that was previously developed by Doumit et al. [12] and used for this research.

(a)

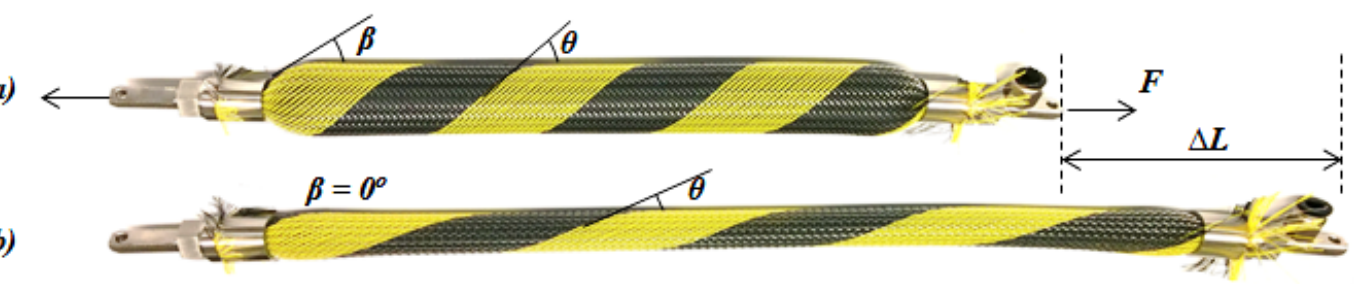

Figure 2.12: PAM prototype a)Pressurized state b)Deflated state

With reference to Figure 2.12, $\theta$ is the braid angle of the sleeve, $\beta$ is the approximate sleeve inclination angle at muscle ends, $\Delta L$ is the muscle contraction distance, and $F$ is the muscle force. The muscle operation is simple, as the bladder is inflated, using an external fluid flow through the end fixture, the muscle pressure increases and results in two types of stresses acting on the muscle's thin inner walls, namely, hoop and longitudinal stresses [35]. The hoop stresses will yield to a force that would radially expand the muscle. Subsequently due to the sleeve's structural properties 
this will longitudinally contract the muscle. The longitudinal stresses will yield to a force that will longitudinally stretch (relax) the muscle. An unbalance of these two forces (radial and longitudinal) yields to a muscle motion such as contraction or relaxation. Neglecting all other forces such as friction, the maximum contraction distance of the muscle coincides with the equilibrium state of both radial and longitudinal forces. If the muscle contraction is externally resisted, the muscle produces a substantial contraction force that is called the muscle force. However, the muscle does not produce any expansion force if the relaxation motion is resisted, and thus the PAM is a unidirectional actuator.

The distinctive properties of the PAM also make it very appealing for the biomedical field. It has been used for human motion rehabilitation devices [36, 37]. This is attributed to the PAM compliant behaviour, high force to weight ratio, high contraction ratio as well as its non-linear force vs elongation relationship.

\subsubsection{Existing PAM Models}

A literature survey has shown that the PAM has been extensively studied and tested by researchers. Most models developed to predict the PAM examine either the static force generation or the dynamic contraction behaviour [38],[39]. Few if any models exist to predict the behaviour of a PAM when it is inflated, then sealed and used as a passive air spring.

The first PAM analytical model was developed by Gaylord [38] for the muscle force F in static condition and later by Schulte et al. [40] as follows:

$$
F=\frac{P \pi D_{90^{\circ}}^{2}}{4}\left(\cos ^{2} \theta-1\right)
$$

where $P$ is the muscle pressure, and $D_{90}$ is the diameter when the braid angle $\theta$ is $90^{\circ}$. Many similar static models were later developed by other researchers such as Chou et al. [41]. The aforementioned static model was modified by Tondu et al. [42] by including a PAM dynamic force $F_{d y n}$ for the case of the PAM suspended vertically. This term takes into consideration the static muscle force and the friction forces. The model's governing equation is:

$$
F_{d y n}-m g=m \ddot{x}
$$

where $m$ is the mass of the hanging load, $g$ is the gravitational constant and $x$ is 
the muscle contraction distance. The parameters of this lumped model are selected and adjusted based on experimental results. Due to model simplification, Tondu and Lopez have noted that the model given by (2.2.2) is less accurate when the muscle pressure is low $(<200 \mathrm{kPa})$.

Thongchai, et al. [39] adopted a frequency modelling approach to predict the dynamic behaviour of a PAM set. Using the Intelligent Machine Architecture (IMA) framework, they succeeded in developing three linear third order dynamic models, each for a specific frequency range. However, the accuracy of such models could not be determined since the open loop results of the developed models were not reported.

Kang et al. [43] also developed a model to analyze the frequency response of PAM. This model uses Gaylord's model, (2.2.1), with a pressure dependent correction factor $q$.

$$
q p_{g}=1+c_{q 1} \exp \left(c_{q 2} p_{g}\right)
$$

where $c_{q 1}$ and $c_{q 2}$ are experimentally estimated constants. In this model the fiber to fiber friction was modelled as the combination of coulomb and viscous friction forces.

$$
F_{\text {friction }}=c_{v} \dot{\epsilon}+c_{c} \operatorname{sgn}(\dot{\epsilon})
$$

where $\epsilon$ is the muscle strain, $c_{v}$ and $c_{c}$ are experimentally obtained coefficients for the viscous and coulomb friction, respectively. Experimental testing of the model reported that predicted and experimental actuation forces have similar trends but different magnitudes [43].

Reynolds et al. [44] developed a PAM dynamic model based on the Voigt Viscoelastic model. This is a lumped parameter model of the following form:

$$
M \ddot{y}+B \dot{y}+K y=F_{c e}-M g
$$

where $M$ is the load mass, $B$ is the system damping coefficient, $K$ is the spring stiffness constant, and $F_{c e}$ is the effective force provided by the contractile element. The values of these parameters were identified experimentally for a step perturbation of the load at constant pressure. Two lumped parameter models were derived to predict the PAM motion in the contraction and in the relaxation phases. The developed models were experimentally validated for a triangular wave input of muscle pressure between $55 \mathrm{kPa}$ and $124 \mathrm{kPa}$. Reynolds et al. determined their model's error accuracy to be $15 \%$ with respect to the average muscle contraction length. Cao et al. [45] have subsequently recreated and extended the model created by Reynolds et al. They 
examined the dynamic behaviour of PAM from 60 to $500 \mathrm{kPa}$. Utilizing a first order polynomial curve fit, they determined linear pressure dependent equations for the force $F$, spring and damping coefficients $K$ and $B$. From this analysis it was discovered that the experimentally obtained coefficient plots (force, spring, and damping) displayed a drastic change in slope when the pressure reached $200 \mathrm{kPa}$. To address this, Cao et al. proposed two models. The first being a quadratic polynomial model:

$$
F(P)+B_{1}(P) \dot{x}+K_{1}(P) x+K_{2}(P) x^{2}+B_{2}(P) \dot{x} x=L+M \ddot{x}
$$

where $L$ is the contraction force, $x$ is the displacement and $M$ is the mass. The second model was referred to as Piecewise model which is the summation of two equations. One equation describes the system before the $200 \mathrm{kPa}$ limit, and the second equation after $200 \mathrm{kPa}$. This second model displayed a high correlation with the experimental data. Both of these models were then used on a second PAM of slightly different size. Both showed similar results to the first test and appear to bring the model developed by Reynolds et al. [44] much closer to the measured values.

Based on the work done by Tondu et al. [42], a number of modified models have been created. One such model was presented by Itto et al. [46]. They developed a hybrid model with a focus on a mass flow model that converges to zero at steady state. The governing mass flow equation is:

$$
\dot{m}(t)=\alpha(t) \dot{m}_{i}(t)-(1-\alpha(t)) \dot{m}_{o}(t)
$$

where $\alpha$ is a ratio $\in[0,1]$ that is a function of the valve command voltage, $m_{i}(t)$ and $m_{o}(t)$ are the mass flow in and out of the PAM, respectively. Itto et al. used the first law of thermodynamics and the principle of polytropic gases to develop a number of mass flow equations that are applied individually in the form of "if then" statements depending on the pressure in the PAM. However due to the complexity of the modelling, the volume of the muscle was approximated by a second order polynomial based on experimentally obtained constants. In a resulting paper Kogiso et al. [47] divided the model into transient and steady state components. In order to validate the model, the effect of each parameter was evaluated through computer simulations.

Tothova et al. have presented a number of papers examining the non-linear behaviour of antagonistic PAM systems. Their model is based on the fiber geometry and muscle pressure. Their basic geometric model assumes a cylinder with zero wall thickness and a constant diameter. This model is shown in a paper by J. Borzikova et al. [48]. From this model, Tothova et al. [49] developed what they call the advanced 
geometric muscle model. This new model assumes the diameter to be variable with the length of the muscle. The ideal gas law with the assumption of constant temperature and the principle of virtual work give the following force equation:

$$
F=-p \frac{d V}{d h}
$$

or

$$
F=-p\left(\frac{\pi d_{1}^{2}}{20}+\frac{8 L^{2}-6 h^{2}}{15 \pi N^{2}}+\frac{d_{1} \sqrt{4 L^{2}-h^{2}}}{15 N}-\frac{d_{1} h^{2}}{15 N \sqrt{4 L^{2}-h^{2}}}\right)
$$

where $p$ is the muscle pressure, $L$ is half of the length of a single fiber, $h$ is the muscle length, $N$ is the number of windings, $d_{1}$ is the diameter of the valve. Next the Bernoulli equation is used to create the following flow equation [49].

$$
Q=f_{v} C_{q} \sqrt{\frac{2}{\rho}} A_{v} \sqrt{P_{\text {in }}-\text { Pout }}
$$

where $f_{v}$ is a direction coefficient, $C_{q}$ is the flow coefficient at a pressure drop of 100 $\mathrm{kPa}, P_{\text {in }}-P_{\text {out }}$ is the difference in pressures on either side of the valve (the sign is corrected with $f_{v}$ ). This model was then validated with a computer simulation. Following this, Pitel et al. conducted a physical experiment in order to further evaluate the model. Both the simple and advanced geometric models were simulated along with a lumped parameter model modified from Hill's muscle model [50]. The experimental set up consisted of two PAM in an antagonistic position connected to a weighted arm. An imbalance in the pulling forces of the muscles would be seen as an angular deviation of the arm. It was concluded that the advanced geometric model was the most accurate, with a mean absolute error of $1.12^{\circ}$ which corresponds to a relative error of $4.74 \%$. This model assumes the muscle to be a cylinder and uses Bernoulli equation to calculate the flow through the valves which is only valid for low velocities when the gas is assumed incompressible. This may lead to error when examining muscles with higher pressure and flow rates.

Sorge, [51] used a Newtonian approach to create a complex model of PAM. The model begins by introducing the axial and longitudinal deformation of the bladder into the conventional Gaylord force model in (2.2.1). The braid tension is refined by examining the texture effect of the overlapping fibers. A rotational friction moment in the sheath causes fibers to deviate from their centerlines and add to the previously obtained equations. The main contribution of this article is a complex equation describing the total force of the muscle. The dynamic aspect of this model is however somewhat limited. The force equilibrium equation (2.2.2) from Tondu and Lopez [42] 
is used. The flow through inlet or outlet orifices is described as a ratio of upstream and downstream pressures. By using the polytropic law and deriving with respect to time the following filling equation is obtained:

$$
\dot{p}=\frac{n p}{V}\left[\left(\frac{G_{\text {in }}-G_{\text {out }}}{\mu_{o}}\right)\left(\frac{p_{o}}{p}\right)^{\frac{1}{n}}-\dot{V}\right]
$$

where $n$ is the polytropic exponent, $G$ is the flow rate, $V$ is the volume and the subscript $X_{0}$ indicates a reference state. The model is then compared to the experimental data collected by Chou et al. [41] as well as Tondu et al. [42]. The model was found to be in accordance with experimental results.

Tri Vo-Minh et al. [52] used the Maxwell-slip model to describe the hysteresis in PAM. The authors used a lumped parameter model with several parallel Maxwellslip elements. Each element is determined by a stiffness $k$ and a saturation force $\mathrm{w}$ parameter. The hysteric output force then becomes the summation of the individual Maxwell-slip elements:

$$
F_{\text {hysout }}=\sum_{1}^{n} F_{i}\left(w_{i}, k_{i}\right)
$$

Conducting isometric muscle contraction testing, values of the stiffness and saturation force parameters were determined. With the summation of four Maxwell slip elements this model accurately recreated the total hysteresis of a specific muscle after experimental testing. Although advanced control systems such as these can partially compensate for frictional losses and hysteresis, the development of an accurate PAM model would greatly simplify the control scheme requirements. 


\section{Chapter 3: PAM Modelling and Simulation}

\subsection{PAM modelling}

The PAM dynamic model is an analytical relationship that permits the designer to simulate the behaviour of the muscle as a function of time. In current and previous models found in literature, the PAM is typically examined while it is being actively inflated or deflated. There are however no models that specifically aim to examine the passive stretch and relaxation cycle of a pre-inflated PAM. In this work, a Newtonian based approach [35] was used as a starting point upon which two additional parameters were considered. First, the isothermal gas assumption was replaced with a polytropic gas model. Furthermore, many models are inaccurate at low pressures, this work also examined the stress in the bladder material and the effect it had on the muscle force generation in low pressure cases.

\subsubsection{Force model}

The base model developed by Doumit [35], assumes that the muscle is composed of three shape elements; a frustum cone that models each muscle end and a cylinder to model the middle section. These muscle characteristics are illustrated in Figure 3.1 


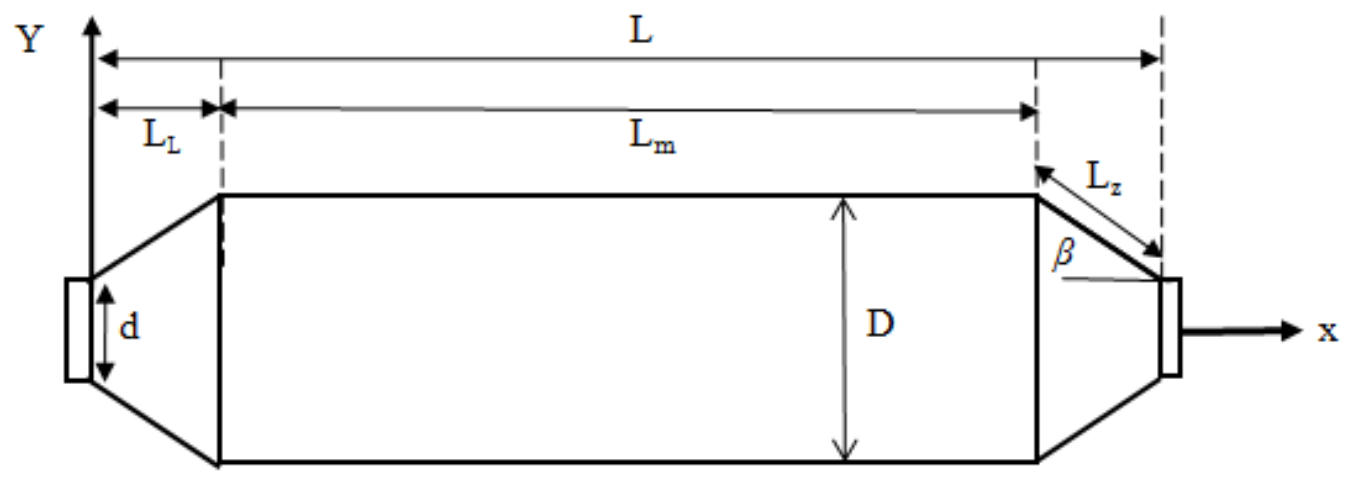

Figure 3.1: General PAM structure

With reference to Figure 3.1, $D$ is the muscle diameter, $d$ is the muscle end diameter, $L$ is the overall muscle length, $L_{m}$ is the muscle middle section length, $L_{L}$ is the horizontal length of the cone and $L_{z}$ is the cone generator length. Based on this muscle's structure, the muscle total volume $V_{\text {total }}$ is derived as:

$$
V_{\text {total }}=\frac{2}{3} \pi L_{L}\left[\left(\frac{D}{2}\right)^{2}+\left(\frac{d}{2}\right)^{2}+\frac{d D}{4}\right]+\pi \frac{D^{2}}{4}\left(L-2 L_{L}\right)
$$

This volume includes the volume occupied by the bladder material but not the volume occupied by the mesh, therefore the internal gas volume is given by:

$$
V=V_{\text {gas }}=V_{\text {total }}-V_{\text {bladder }}
$$

where $t_{b}$ is the bladder thickness

$$
V_{\text {bladder }}=L \pi\left(\left(\frac{D}{2}\right)^{2}-\left(\frac{D}{2}-t_{b}\right)^{2}\right)
$$

moreover, the force $F_{m}$ produced by the muscle is derived as [35]:

$$
F_{m}=N T_{f} \cos \theta_{e} \cos \beta
$$

where $N$ is the number of fibers in the muscle's sleeve, $\theta_{e}$ is the end muscle braid angle, $\beta$ is the sleeve inclination angle at the muscle ends, and $T_{f}$ is the tension within muscle's fibers which is given by:

$$
\overrightarrow{T_{f}}=\overrightarrow{T_{h 1}}+2 \vec{T}_{h 2}-\vec{T}_{l}
$$


where $T_{l}$ is the fiber tension resulting from the longitudinal stresses, $T_{h 1}$ and $T_{h 2}$ are the fiber tension resulting from the hoop stresses in the cylindrical and two cone frustum sections, respectively.

$$
\begin{gathered}
T_{h 1}=\left[\frac{P\left(D-2 t_{b}-2 t_{f}\right)-2 \sigma_{b h} t_{b}}{2 n N \sin \theta}\right] L_{1} \\
T_{h 2}=\left[\frac{P\left(D+d+4 t_{b}-4 t_{f}\right) \cos \beta-4 \sigma_{b h} t_{b}}{4 n N \sin \theta_{a}}\right] L_{Z} \\
T_{l}=\frac{\left[P\left(\frac{D}{2}-t_{f}-t_{b}\right)^{2}-\sigma_{b l} D t_{b}\right]}{N D \cos \theta} \frac{L}{n} \tan \theta
\end{gathered}
$$

where $\sigma_{b h 1}$ is the stress in the bladder in the circumferential direction, $P$ is the gas pressure. The circumferential stress is also often called the hoop stress. No distinction is made between the bladder stress in the cylindrical and frustum sections. The variable $\sigma_{b l}$ is the longitudinal stress in the bladder. Variables $t_{f}$ and $t_{b}$ are the thickness of an individual fiber and the thickness of the bladder respectively. The variable $\theta_{a}$ is the average braid angle, which is defined as the mean value between the muscle end braid angle and the cylindrical section braid angle.

\subsubsection{Bladder Stress}

Previous PAM testing achieved by Doumit [35], and other authors, [42] [45], demonstrated that the present PAM models are not accurate at low pressures. It is theorized that this may be due to the bladder material. At high pressures, the amount of force required to elongate the bladder material is negligible when compared to the overall tensile force produced by the PAM as a whole. However, at lower pressures

the force required to elongate the bladder represents a larger portion of the total PAM force. For this reason, the mechanical properties of the bladder material were examined more closely. This section describes how the material was tested biaxially in order to generate polynomial curves describing the bladder stress which were subsequently incorporated into the model. 


\section{Biaxial Stress Testing}

Previously, the model by Doumit [35], incorporated the mechanical behaviour of the bladder using uniaxial experimental tests and it was assumed that the material was isotropic. In order to improve these assumptions, biaxial tensile testing was carried out.

During this testing, samples of bladder material were stretched following identical PAM specific deformations. When the PAM is inflated from rest, the diameter increases while the length decreases. Therefore, the bladder material is stretched in one direction, to accommodate the increased diameter, while being compressed in the other direction. This bladder strain is also present when the PAM is being used as a passive air spring. To determine the strain in the bladder material during operation, the underlying assumptions used in the development of the force model were used. Figure 3.2 shows the geometrical relationship between the fibers and the muscle. In the image, a single fiber is unwrapped and positioned next to the muscle.

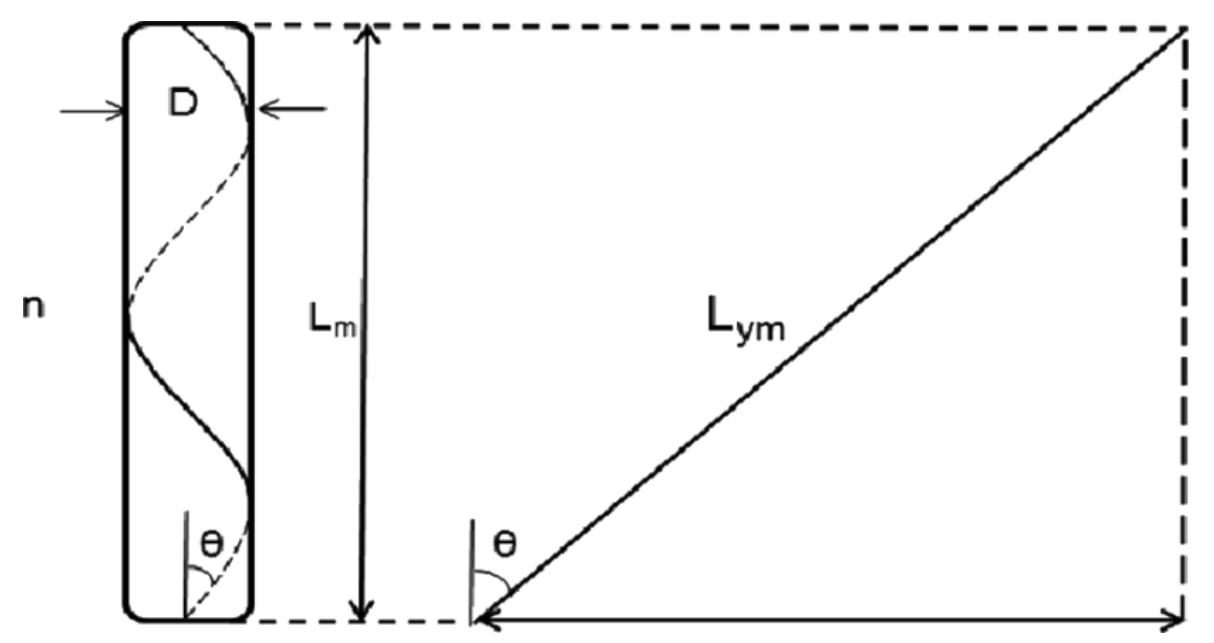

Figure 3.2: Geometrical properties of the braided mesh that reflects the muscle middle section. Image and caption taken from [35]

where $n$ is the number of fiber revolutions, in this case $n=1$. Variables $D, L_{m}$ and $\theta$ are the muscle diameter, muscle length (for the cylindrical center section) and the braid angle. Variable $L_{y m}$ is the length of the fiber itself. According to this model, the relationships between the braid angle and the muscle dimensions is given as:

$$
D=\frac{L_{y m} \sin \theta}{\pi n}
$$




$$
L_{m}=L_{y m} \cos \theta
$$

combining (3.1.9) and (3.1.10) the braid angle can be eliminated giving an alternate form to the diameter equation.

$$
D=\frac{\sqrt{L_{y m}^{2}-L_{m}^{2}}}{\pi n}
$$

The braid angle of the PAM depends on a number of factors including the fiber diameter, number of fibers as well as the tightness of the weave. The PAM used in this work was made with a Flexo Pet meshing by Techflex Braided Sleeving Solutions. The relaxed and fully contracted angles for this mesh were measured to be $25^{\circ}$ and $50^{\circ}$ using a goniometer. Using these two braid angles as well as (3.1.9), (3.1.10) and (3.1.11) the theoretical maximum strain in the longitudinal and circumferential directions of the bladder were calculated for three different muscle lengths. These values are presented in Table 3.1. The variable $n$ refers to the number of fiber revolutions. Therefore, a $n 2$ muscle has 2 full fiber revolutions. Strain $L$ and Strain $C$ refers to the bladder strains in the longitudinal and circumferential (hoop) directions respectively. For future reference in this work, the strain values obtained via the braid angle and the equations derived by [35] will be referred to as the theoretical strains.

Table 3.1: Geometric properties of PAM and theoretical strain of elastic bladder based on braid angle equations.

\begin{tabular}{cccccc}
\hline PAM & Variable & Relaxed state & Contracted state & Strain $L$ & Strain $C$ \\
\hline \hline & $D$ & $15.45 \mathrm{~mm}$ & $28.78 \mathrm{~mm}$ & & \\
$\mathrm{n} 1$ & $L_{m}$ & $10.7 \mathrm{~cm}$ & $7.58 \mathrm{~cm}$ & -0.290 & 0.863 \\
& $\theta$ & $25^{\circ}$ & $50^{\circ}$ & & \\
\hline \multirow{5}{*}{$\mathrm{n} 2$} & $D$ & $15.45 \mathrm{~mm}$ & $29.06 \mathrm{~mm}$ & & \\
& $L_{m}$ & $21.6 \mathrm{~cm}$ & $15.32 \mathrm{~cm}$ & -0.290 & 0.881 \\
& $\theta$ & $25^{\circ}$ & $50^{\circ}$ & & \\
$\mathrm{n} 3$ & $D$ & $15.45 \mathrm{~mm}$ & $29.32 \mathrm{~mm}$ & & \\
& $L_{m}$ & $32.7 \mathrm{~cm}$ & $23.19 \mathrm{~cm}$ & -0.290 & 0.898 \\
& $\theta$ & $25^{\circ}$ & $50^{\circ}$ & & \\
\hline
\end{tabular}


To confirm the calculated maximum theoretical strain values, the three PAM were measured using digital callipers in their deflated and inflated states. The muscles were inflated until they reached a maximum diameter and higher inflation pressure did not cause further contraction. Table 3.2 presents the measured strain values. Strain $L$ and Strain $C$ refers to the bladder strains in the longitudinal and circumferential (hoop) directions respectively. The strain values obtained from the measurements of the physical PAM will be referred to as the experimental strain values.

Table 3.2: Geometric properties of PAM and experimental strain of elastic bladder obtained using physical measurements.

\begin{tabular}{cccccc}
\hline PAM & Variable & Relaxed state & Contracted state & Strain L & Strain C \\
\hline \hline & $D$ & $15.45 \mathrm{~mm}$ & $29.5 \mathrm{~mm}$ & & \\
$\mathrm{n} 1$ & $L_{m}$ & $10.7 \mathrm{~cm}$ & $8.3 \mathrm{~cm}$ & -0.224 & 0.909 \\
& $\mathrm{P}$ & $0 \mathrm{kPa}$ & $138 \mathrm{kPa}$ & & \\
\hline \multirow{4}{*}{$\mathrm{n} 2$} & $D$ & $15.45 \mathrm{~mm}$ & $29.5 \mathrm{~mm}$ & & \\
& $L_{m}$ & $21.6 \mathrm{~cm}$ & $16.0 \mathrm{~cm}$ & -0.259 & 0.909 \\
& $\mathrm{P}$ & $0 \mathrm{kPa}$ & $276 \mathrm{kPa}$ & & \\
$\mathrm{n} 3$ & $D$ & $15.45 \mathrm{~mm}$ & $29.8 \mathrm{~mm}$ & & \\
& $L_{m}$ & $32.7 \mathrm{~cm}$ & $23.6 \mathrm{~cm}$ & -0.278 & 0.929 \\
& $\mathrm{P}$ & $0 \mathrm{kPa}$ & $345 \mathrm{kPa}$ & & \\
\hline
\end{tabular}

From Tables 3.1 and 3.2, the maximum strain ratios obtained were similar but not identical. The cause of the difference is unclear, therefore, both of the strain ratios were tested. For the theoretical case, the maximum longitudinal strain is -0.29 and the maximum circumferential strain is 0.9. For the experimental case, the longitudinal and circumferential strains are -0.28 and 0.93 respectively.

The next test parameter considered was the strain rate. It has been previously shown that the elongation rate of a PAM has little effect on its mechanical properties and stiffness [11]. However, that conclusion was drawn following elongations tests made at pressures above $200 \mathrm{kPa}$ and may not be entirely valid at lower pressure. Therefore multiple strain rates were examined for the bladder material elongation tests. The strain rates were chosen based on the capabilities of the testing machine.

The biaxial testing machine used was a BioTester Biaxial Test System by CellScale 
biomaterials testing [53]. The machine has four arms all pointed towards the center of the unit, see Figure 3.3. On each arm a set of five tines is held by a magnet. These tines are called rakes. A sample is mounted between the rakes, the arms then move in a pre-programmed pattern to deform the sample. The arms are instrumented with a $25 \mathrm{~N}$ load cell.

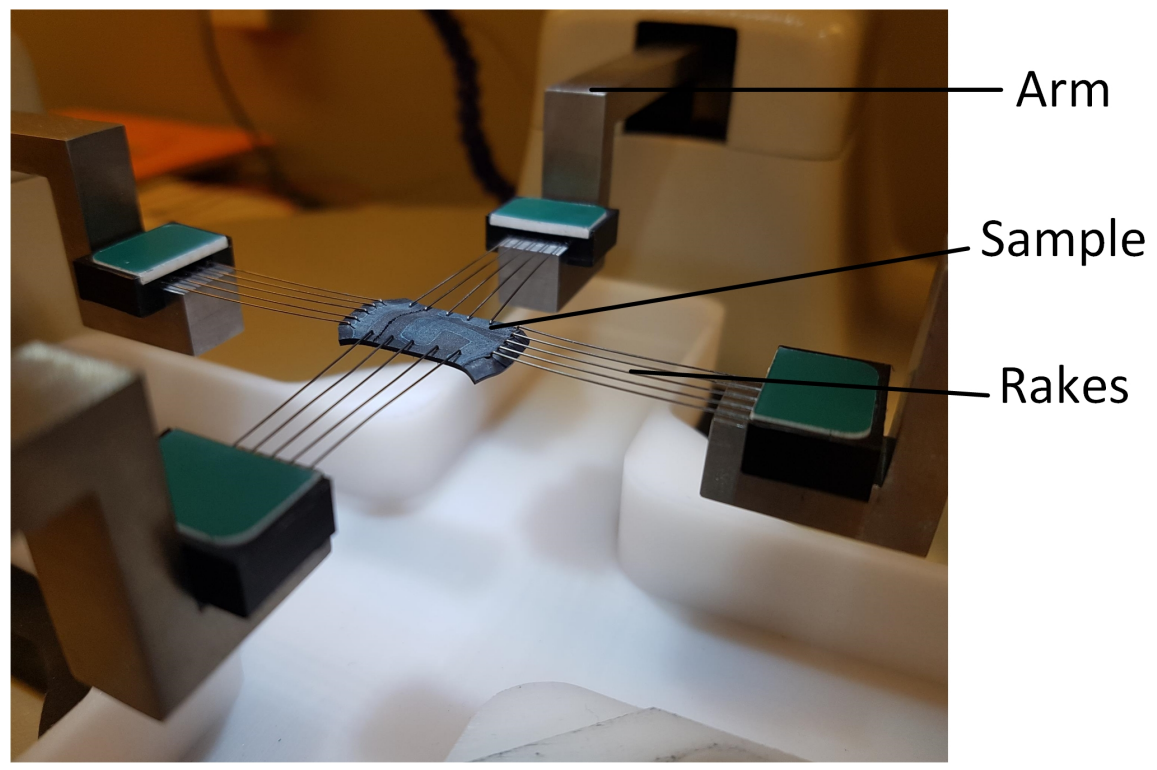

Figure 3.3: Photograph of the biaxial testing machine. The sample is suspended in midair between the four instrumented arms.

The software driving the device allows the user to set the desired strain in the $X$ and $Y$ axes as well as the duration of one cycle. The machine was programmed to move using percentages of the initial specimen dimensions. A ramp up to the final desired elongation value was used. The rakes moved from their initial position to the prescribed position so that the maximum displacement of both axes was reached simultaneously. The actuated arms of the device can perform up to 1 cycle every 2 seconds, however excessively high speed may tear the sample. Furthermore, the tines holding the sample are connected to the instrumented arms of the machine by small magnets. At high speeds these magnetic connections shake, adding noise to the data. For these reasons, the minimum cycle duration was set to be $14 \mathrm{~s}$. With the maximum cycle frequency set, the subsequent frequencies were chosen by doubling the previous frequency resulting in cycle durations of $30 \mathrm{~s}$ and $60 \mathrm{~s}$. The delay between the elongation and recovery phases of the test was set to $0 \mathrm{~s}$. The parameters for the biaxial tests are presented in Table 3.3. 
Table 3.3: Parameters for biaxial bladder elongation tests

\begin{tabular}{cccc}
\hline X / Y strain & Cycles & Extension duration & Recovery duration \\
\hline \hline $0.90 /-0.290$ & 5 & $30 \mathrm{~s}$ & $30 \mathrm{~s}$ \\
& 5 & $15 \mathrm{~s}$ & $15 \mathrm{~s}$ \\
& 5 & $7 \mathrm{~s}$ & $7 \mathrm{~s}$ \\
\hline $0.93 /-0.278$ & 5 & $30 \mathrm{~s}$ & $30 \mathrm{~s}$ \\
& 5 & $15 \mathrm{~s}$ & $15 \mathrm{~s}$ \\
& 5 & $7 \mathrm{~s}$ & $7 \mathrm{~s}$ \\
\hline
\end{tabular}

Each sample consisted of a $15 \mathrm{~mm}$ by $15 \mathrm{~mm}$ specimen of bladder material. The bladder material is a butyl rubber bicycle tube. The rakes were set to form an $11 \mathrm{~mm}$ square leaving approximately $2 \mathrm{~mm}$ of material beyond the rakes on each side. The sample was then positioned in the machine so that the longitudinal axis was aligned with the $\mathrm{Y}$ axis of the machine. While being supported from underneath, the rakes were carefully pushed into the sample as far as they would go. The tines of the rakes punctured the material and protruded out the bottom of the sample. The support was then removed leaving the sample suspended in midair between the 4 sets of rakes, this can be seen in Figure 3.3. The rakes used had a tine diameter of $305 \mu \mathrm{m}$, a tine spacing of $1.7 \mathrm{~mm}$ and a puncture depth of $1.9 \mathrm{~mm}$. The material had a thickness of $0.9 \mathrm{~mm}$

The testing procedure consisted of testing each sample at both elongations and all speeds, for a total of 6 consecutive tests per sample. The biaxial deformation can be seen in Figure 3.4. The vertical seam in this image was positioned intentionally to demonstrate that the longitudinal axis of the rubber material (aligned with the seam) was in compression whereas the circumferential direction (perpendicular to the seam) was in elongation

Upon completion of the tests, the force vs displacement data was exported as a .csv file before being loaded into MatLab for processing. The engineering stress and engineering strain were calculated using the initial cross sectional area. The stress vs strain data was then plotted for the longitudinal (A) and circumferential (B) directions. Figure 3.5 presents the stress vs strain relationship of the bladder material in the longitudinal and circumferential directions. From Figure 3.5, the graphs illustrate that the material exhibits hysteresis and a non-linear curve. Furthermore, the outlier 


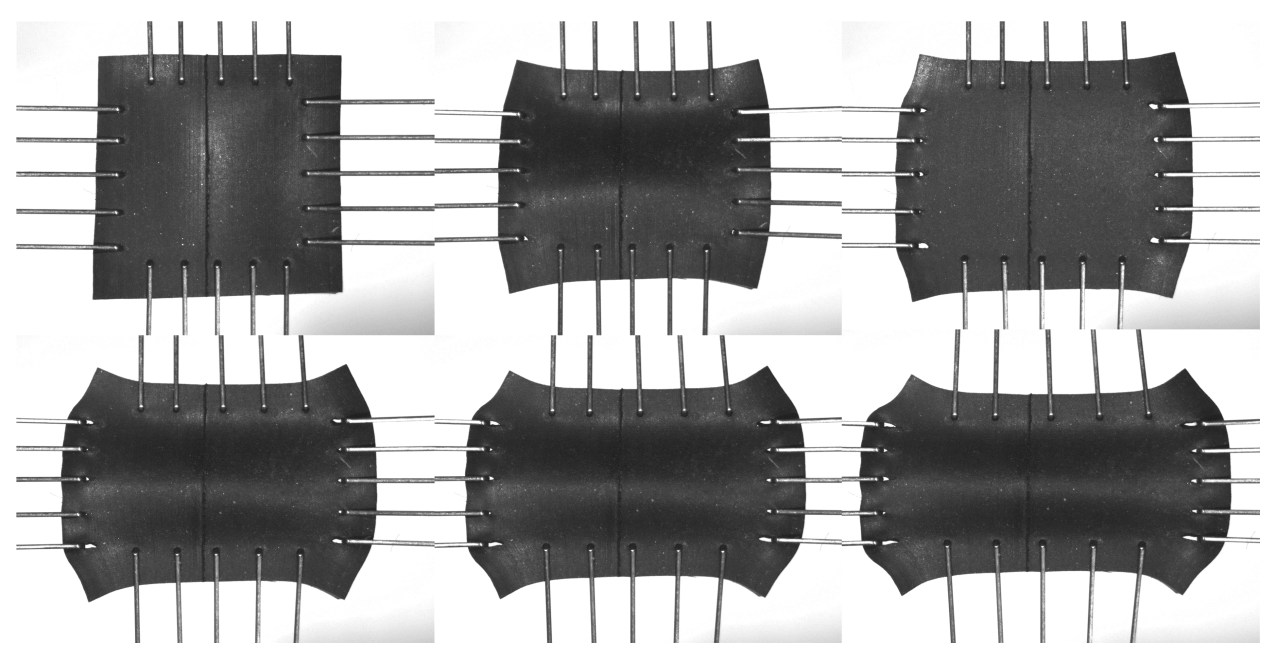

Figure 3.4: Photograph of the biaxial testing of a butyl rubber sample at 6 stages of testing.

visible in graph B is caused by the very first elongation cycle. The bladder material is known to be visco-elastic and requires multiple cycles before reaching the steady state. This was the primary motivation for the choice of 5 cycles per test. The plot indicates that after the first cycle, all subsequent cycles were very similar, thus, five cycles were indeed sufficient for the material to reach a steady state.
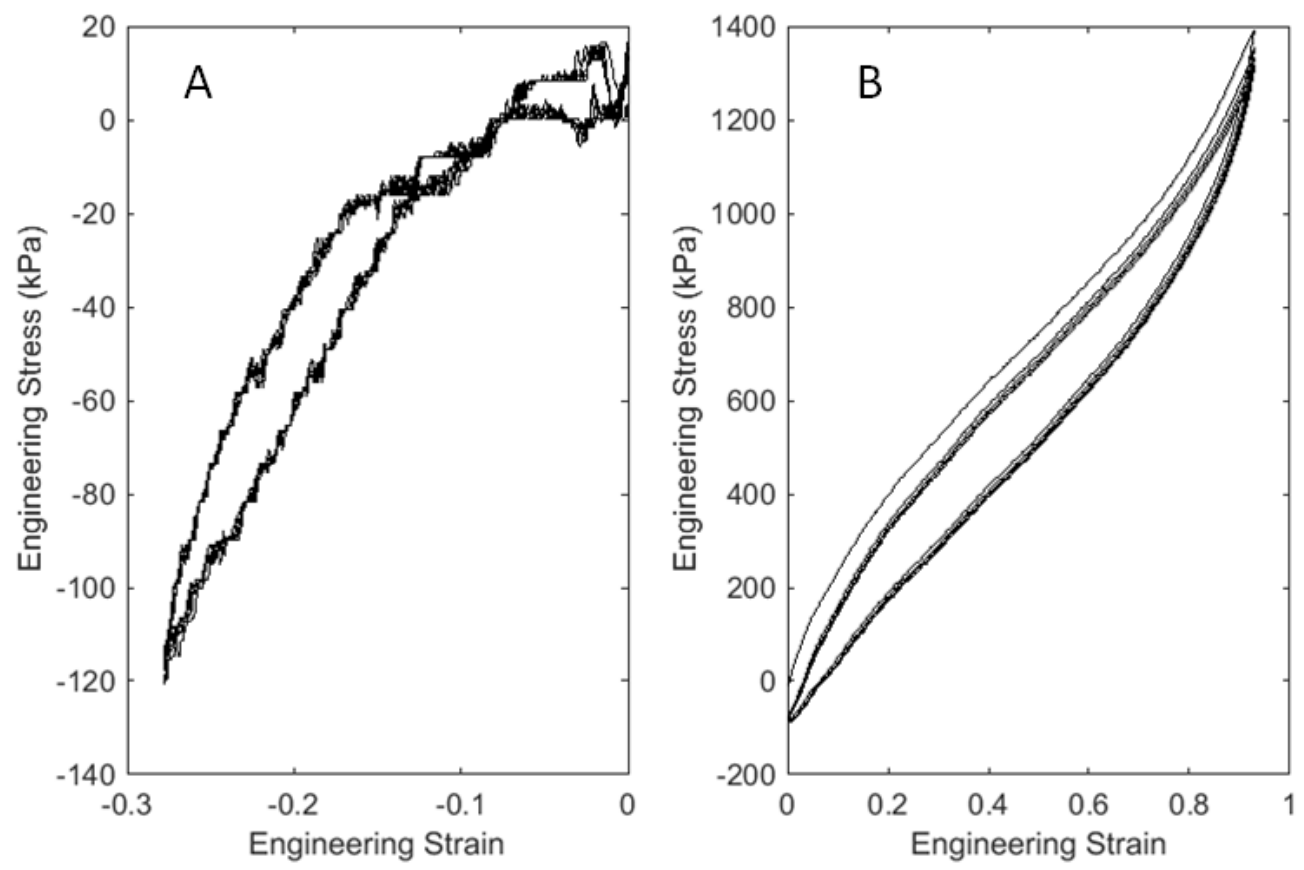

Figure 3.5: Graph of the stress vs strain relationship of the butyl rubber sample 5. Strain ratio of $0.93 /-0.278$, cycle duration of $30 \mathrm{~s}$. A) Longitudinal Stress B)

Circumferential Stress 
A sixth order polynomial was fitted to the data of the fifth cycle for the longitudinal and circumferential strains. Figure 3.6 presents the fifth cycle as well as the fitted polynomial curve.
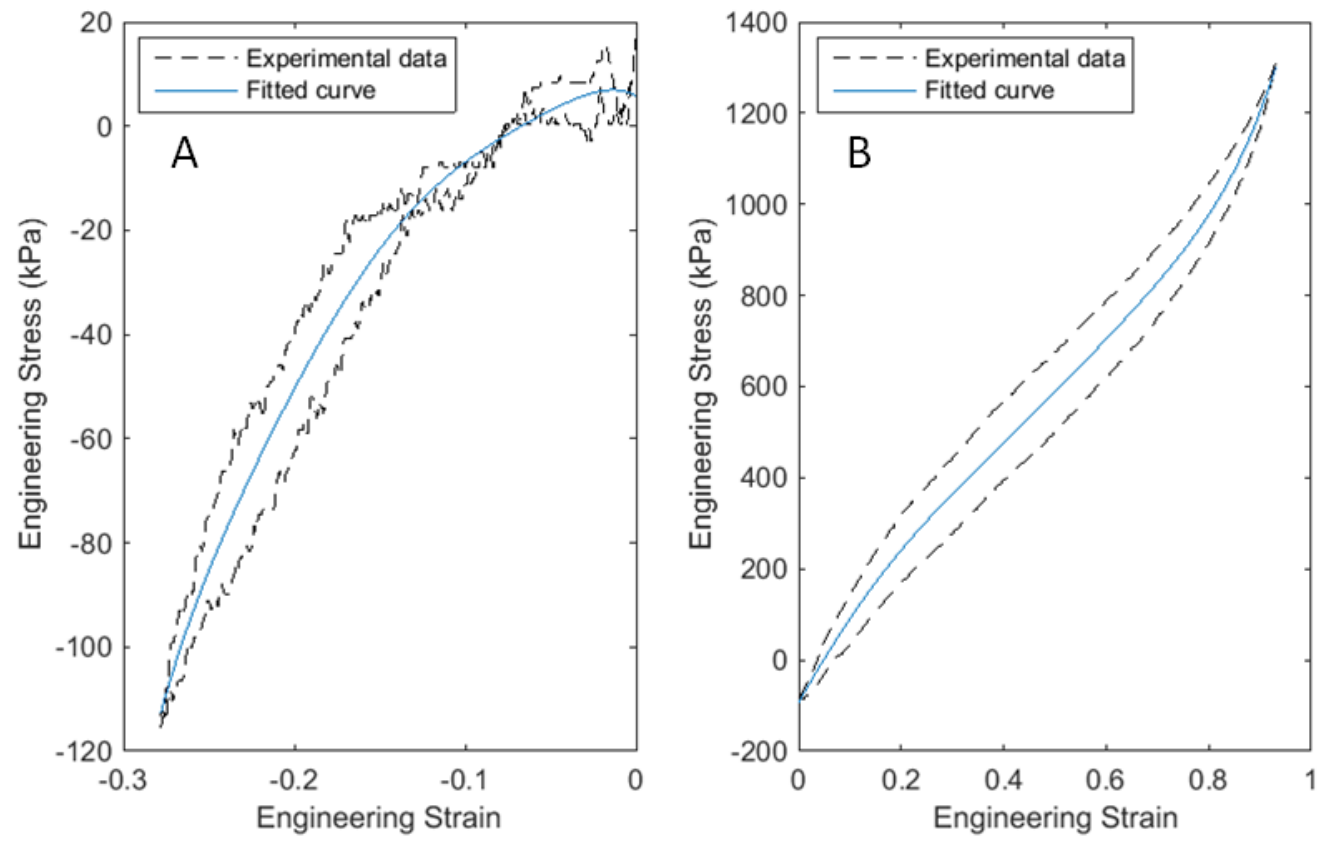

Figure 3.6: Graph of the stress vs strain relationship of the fifth cycle of the butyl rubber sample 5, and the sixth order polynomial curve that was fit to the data. Strain ratio of $0.93 /-0.278$, cycle duration of $30 \mathrm{~s}$. A) Longitudinal Stress B) Circumferential Stress

The fitted curves were compared for various test elongations and speeds. Figure 3.7 presents the superposition of the fitted curves for samples 2 and 3 . The strains in the longitudinal and hoop directions are -0.278 and 0.93 respectively. In the circumferential direction the stress vs strain curves are very similar for both samples and for both testing speeds. In the longitudinal direction however, the $30 \mathrm{~s}$ tests are slightly above the $60 \mathrm{~s}$ curves. For this sample, the $30 \mathrm{~s}$ test was performed immediately after the $60 \mathrm{~s}$ test. This may have caused some deformation of the sample or enlargement of the holes made by the hooks. The deformation of the material near the edges of the sample was most apparent at the corners. At these locations, the holes formed by the tines were stretched more than those in the center of each side. This may have reduced the force measured by the testing machine since the material was not being stretched to the full desired elongation across the entire edge of the sample. This inaccuracy was ignored. 

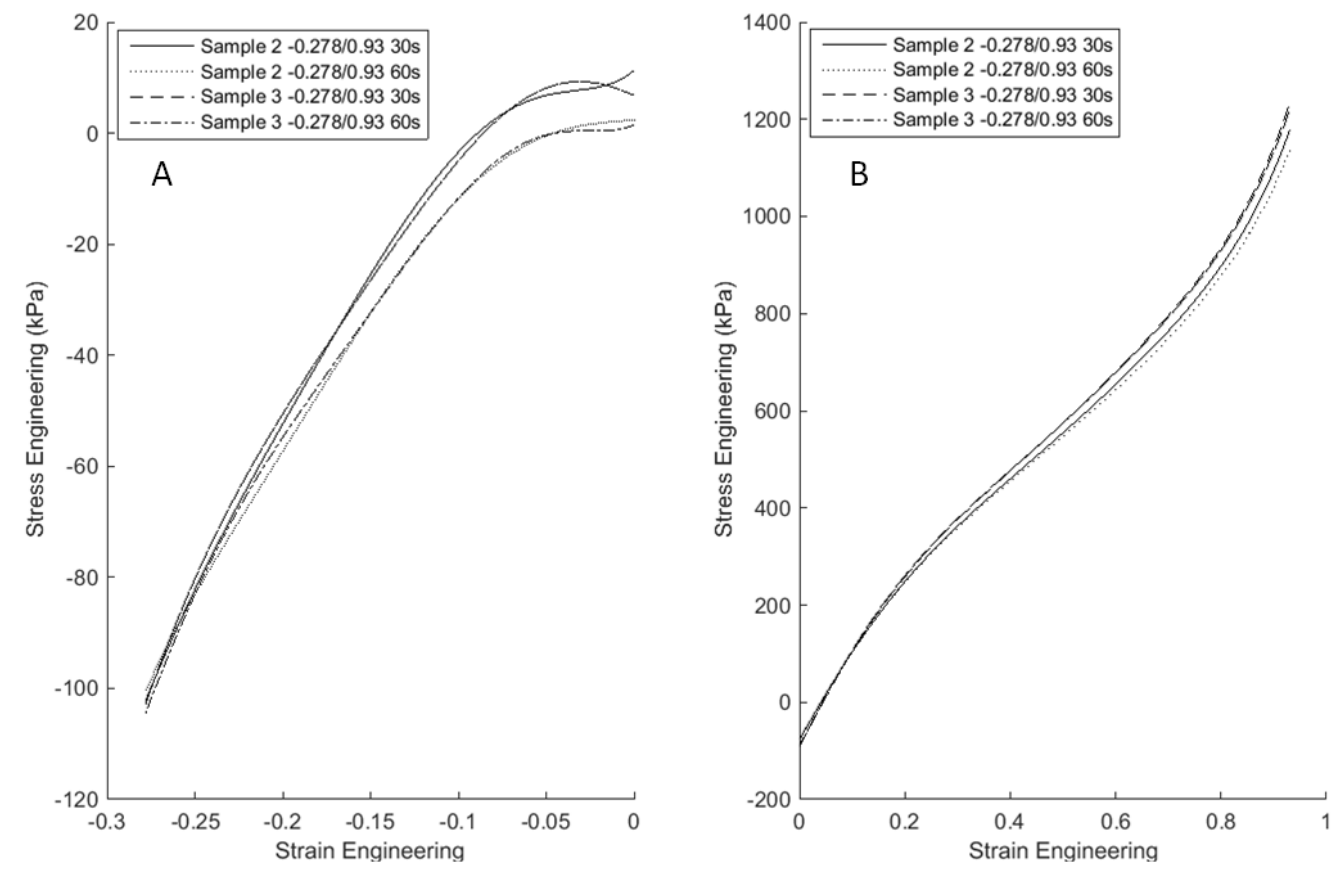

Figure 3.7: Comparison of the fitted curves for samples 2 and 3. Strain ratio of 0.93 / -0.278 , cycle duration of $30 \mathrm{~s}$ and $60 \mathrm{~s}$. A) Longitudinal Stress B) Circumferential Stress

Figure 3.7 presents two samples at two test speeds however all at the same strain ratio. Figure 3.8 however, presents the difference between the theoretical strain ratio $(-0.29 / 0.9)$ and the experimental strain ratio $(-0.278 / 0.93)$. 

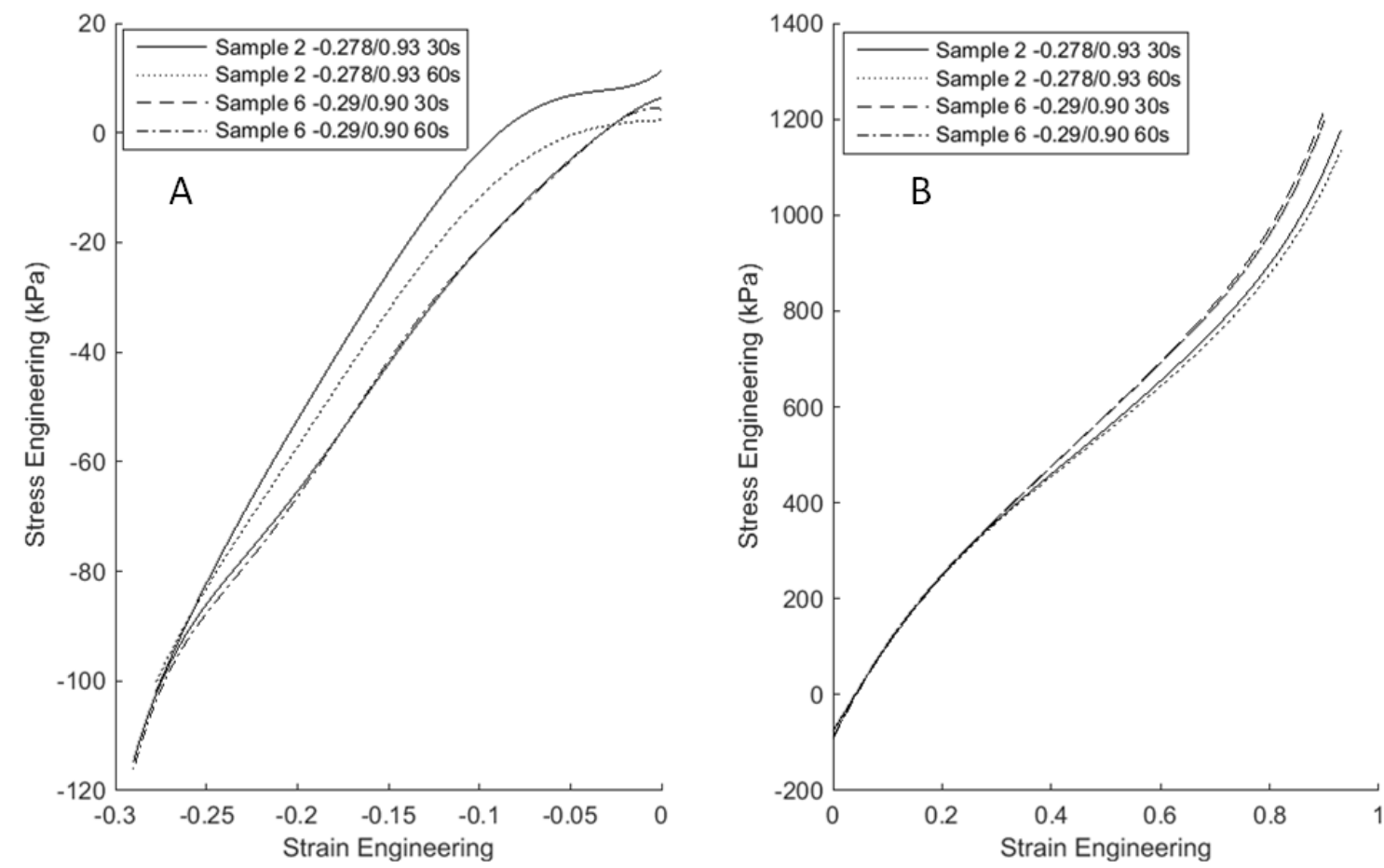

Figure 3.8: Comparison of the fitted curves for samples 2 and 6. Sample 2 was tested at strain ratio of $-0.278 / 0.93$, sample 6 was tested at strain ratio of $-0.29 / 0.90$. A)

Longitudinal Stress B) Circumferential Stress

Although the two strain ratios compared in Figure 3.8 do not match exactly they are similar. Furthermore, the circumferential stress in the bladder (B) is an order of magnitude greater than the stress in the longitudinal direction (A). Since the curves in the circumferential direction match well and are much more important than the longitudinal direction (in terms of magnitude) the inaccuracy of the longitudinal curve fit was overlooked. The polynomial equations for sample number 5 at strain ratio $-0.278 / 0.93$ were chosen to represent the biaxial behaviour of the material in the model. The stress in function of strain equations for the longitudinal and circumferential directions of the butyl rubber bladder material can be seen in (3.1.12) and (3.1.13).

$$
\begin{gathered}
\sigma_{b l}=-2018400 \epsilon_{L}^{6}-1553000 \epsilon_{L}^{5}-438900 \epsilon_{L}^{4}-52700 \epsilon_{L}^{3}-3700 \epsilon_{L}^{2} \\
\sigma_{b h}=11166 \epsilon_{H}^{6}-24717 \epsilon_{H}^{5} 19462 \epsilon_{H}^{4}-4759 \epsilon_{H}^{3}-1415 \epsilon_{H}^{2}+2046 \epsilon_{H}-81
\end{gathered}
$$

where $\sigma_{\text {Long }}$ and $\sigma_{\text {Hoop }}$ are the bladder stresses, in $\mathrm{kPa}$, in the longitudinal and circumferential directions respectively. Variables $\epsilon_{L}$ and $\epsilon_{H}$ are the strains in the longitudinal and circumferential directions respectively. These equations are added to force model 
equations (3.1.6), (3.1.7), (3.1.8).

\subsubsection{Pressure model}

The PAM is a closed system containing a gas that undergoes compression and expansion. The pressure of the gas was modelled using the polytropic gas assumption.

$$
P V^{n g}=\text { constant }
$$

where $P$, and $V$ are the gas pressure and volume respectively, $n g$ is the polytropic index and varies from 1.0, for the isothermal case to 1.4, for the adiabatic case. In this work, the PAM was inflated with atmospheric air and sealed before being put through stretch/relaxation cycles. Therefore, (3.1.14) can be re-written as:

$$
P V^{n g}=\text { constant }=P_{\text {initial }} V_{\text {initial }}^{(n g)}
$$

or

$$
P=\frac{P_{\text {initial }} V_{\text {initial }}^{(n g)}}{V^{n g}}
$$

where $P_{\text {initial }}$ and $V_{\text {initial }}$ are the pressure and volume of the gas when the PAM is inflated but before it is stretched. Variables $P$ and $V$ are the instantaneous values of gas pressure and volume as the muscle is stretched.

\subsubsection{Temperature model}

Assuming the gas inside the PAM to be an ideal gas, the perfect gas law can be used.

$$
P V=m R T
$$

where $m$ is the mass of gas, $R$ is the gas constant and $T$ is the temperature. Combining (3.1.16) and (3.1.17) gives

$$
\frac{T_{\text {initial }}}{T}=\left(\frac{V}{V_{\text {initial }}}\right)^{(n g-1)}
$$

The initial gas temperature, $T_{\text {initial }}$, was assumed to be the room temperature. 


\subsubsection{Friction}

In this work, the frictional force was assumed to be the result of fiber-fiber contact as well as fiber bladder contact. Furthermore, the undulations due to the cross fiber weave were ignored, and cross fibers were assumed to simply lie flat on top of each other. This friction model is part of the model developed by Doumit [54] and is only be summarized in this work. The expression for the fiber-fiber friction force $f_{f r}$ is derived as:

$$
f_{f r}= \begin{cases}-P_{2} A_{f f} \mu_{d} & v<0 \\ \Sigma F_{x}+P A_{f f} \mu_{d} & \Sigma F_{x} \leqslant 0, v=0 \\ P_{2} A_{f f} \mu_{s} & \Sigma F_{x}>0, v=0 \\ P_{2} A_{f f} \mu_{d} & v>0\end{cases}
$$

where $F_{x}$ is the static friction force, $\mu_{s}$ and $\mu_{d}$ are the static and dynamic coefficients of friction, respectively. Such a form, however, is highly non-linear and includes a discontinuity at the transition from the static friction regime to the dynamic one. Such discontinuities are difficult to deal with in numerical simulation. Consequently, for the dynamic case, 3.1.19 was replaced by:

$$
f_{f r d}=P A_{f f} \mu_{d L}
$$

where

$$
\mu_{d L}=\mu_{d} \operatorname{Tanh}\left(K_{f} v\right)
$$

where $f_{f r d}$ is the dynamic frictional force, $\mu_{d L}$ is the new dynamic friction coefficient, $K_{f}$ is a constant that defines how close 3.1.20 approximates the Coulomb friction and $v$ is the PAM contraction velocity. For the results presented in this work, $K_{f}=1$.

In addition to the fiber-fiber friction, friction between the innermost fibers and the bladder was considered. During elongation there is a slight movement of the fibers relative to the bladder. The number of contact surfaces between fibers is expressed as

$$
q=2 n\left(\frac{N}{2}\right)^{2}-\frac{N}{2}
$$

where $N$ is the number of fibers in the mesh and $n$ is the number of full revolu- 
tions done by each fiber. The fiber bladder friction force is written as follows.

$$
F_{f b}=P_{2} \mu_{f b} w\left(L_{y} N-z \frac{q}{2}\right)
$$

where $\mu_{f b}$ is the fiber bladder friction coefficient, $w$ is the width of the fibers, $L_{y}$ is the length of the fibers, and $z$ is the portion of the fiber length that defines the frictional area. Thus contact area between two crossed fibers is

$$
F_{f f}=w * z
$$

\subsubsection{Simulation}

The proposed PAM model was written into the Simulink, MatLab environment, shown in Appendix G. Figure 3.9 presents the block diagram of the simulation. Each block represents a sub function containing multiple equations and variables. The lines between blocks represent variables being passed to different sub functions.

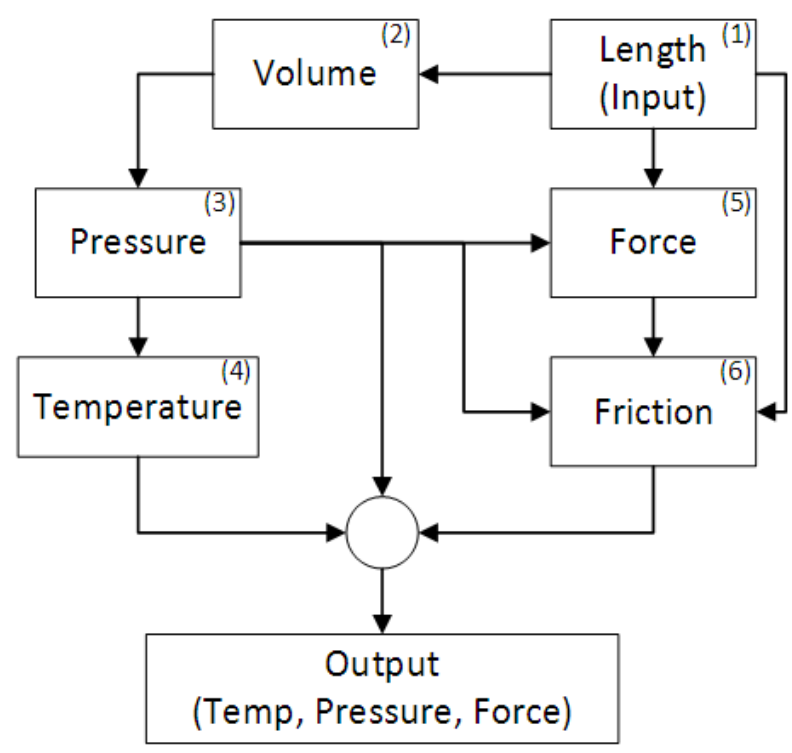

Figure 3.9: PAM simulation block diagram

With reference to Figure 3.9, block \# 1 contains a repeated ramp function that varied from zero to maximum elongation. The muscle length was passed to blocks \# 2 and \# 5. Block \# 2 calculated the volume of the PAM and passed the value to block \# 3 . Block \# 3 calculated the gas pressure and temperature. The PAM pressure and length were passed to block \# 5 which calculated the muscle force. The force was passed to 
block \# 6 which applied the frictional effects before passing along the muscle force. The simulation outputs were the PAM force, pressure and temperature.

\subsection{PAM experimental testing}

In order to validate the proposed PAM model, experimental testing was carried out. The tests were performed using a butyl rubber muscle with the Flexo Pet meshing by Techflex Braided Sleeving Solutions. The muscle had 2 fiber revolutions.

The PAM was tested using an Instron universal testing machine, model 4482. Figure 3.10 shows the PAM in the Instron machine. The machine was programmed to reach a specific elongation while measuring the force that the muscle exerted on the $100 \mathrm{kN}$ load cell.

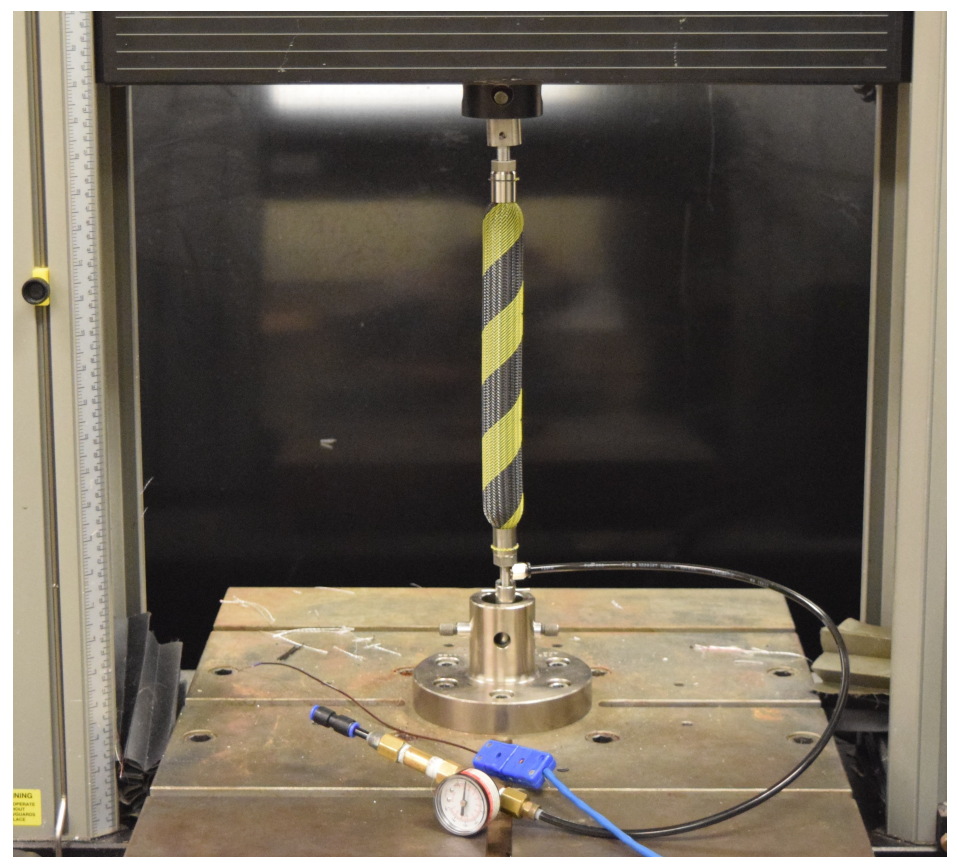

Figure 3.10: PAM prototype in the Instron universal testing machine, model 4482

Due to the limitations of the machine, the PAM was cycled at a maximum speed of $2.5 \mathrm{~mm} / \mathrm{s}$. The data was collected using the Instron machine as well as a pressure transducer and a temperature sensor inserted into the gas chamber of the muscle. Further details can be found in Appendix B 


\subsubsection{Testing Methodology}

The PAM used were custom fabricated in house, specifically for this work. They use a butyl rubber bladder and two revolutions of the Flexo Pet yellow and black meshing. The testing stretched the PAM to $80 \%, 90 \%$, or $95 \%$ of the relaxed muscle length. To calculate these values, the muscles were measured at rest and again once inflated. The difference between these values is the total muscle contraction distance. The $80 \%, 90 \%$ and $95 \%$ are fractions of this value. For the low pressure testing, the PAM was tested exclusively at $95 \%$ elongation since the forces produced by the $80 \%$ and $90 \%$ tests are very small. These measurements as well as others such as the muscle diameter were used as the geometrical inputs required for the simulation.

For the low and the high pressure testing, two different muscles were used. It was thought that the muscle with the integrated temperature sensor might leak. Moreover, if there is a measurable change in temperature caused by the compression of the gas it would be more noticeable in the high pressure situations. Therefore for the low pressure testing a standard (without a temperature sensor) muscle was used. These two muscles were fabricated to be identical, however a slight variation exists and can be observed in the difference in the deflated state of the muscle in Table 3.4. The low pressure muscle was used for the tests at $35 \mathrm{kPa}, 48 \mathrm{kPa}, 55 \mathrm{kPa}, 69 \mathrm{kPa}, 83$ $\mathrm{kPa}$ and $104 \mathrm{kPa}$. The PAM with the internal temperature sensor was used for the tests at $138 \mathrm{kPa}, 207 \mathrm{kPa}$ and $276 \mathrm{kPa}$, this is summarized in Table 3.4. These pressures are those which were measured by visual inspection of the gauge during inflation. The actual pressure recorded by the pressure transducer is slightly different, it is the value read from the transducer that was used in the simulations. The muscle length was measured between the bottoms of the end caps, the diameter was measured using a pair of digital calipers. These are presented in Table 3.5. 
Table 3.4: Geometric measurements of test muscles for high and low pressure testing.

\begin{tabular}{lccc}
\hline Muscle & State & Muscle Length & Muscle Diameter \\
\hline \hline & Deflated $0 \mathrm{kPa}$ & $0.223 \mathrm{~m}$ & $0.02 \mathrm{~m}$ \\
& Inflated $35 \mathrm{kPa}$ & $0.185 \mathrm{~m}$ & $0.02725 \mathrm{~m}$ \\
PAM 1 & Inflated $48 \mathrm{kPa}$ & $0.181 \mathrm{~m}$ & $0.0276 \mathrm{~m}$ \\
& Inflated $55 \mathrm{kPa}$ & $0.18 \mathrm{~m}$ & $0.0278 \mathrm{~m}$ \\
& Inflated $69 \mathrm{kPa}$ & $0.177 \mathrm{~m}$ & $0.0280 \mathrm{~m}$ \\
& Inflated $83 \mathrm{kPa}$ & $0.174 \mathrm{~m}$ & $0.02875 \mathrm{~m}$ \\
& Inflated $104 \mathrm{kPa}$ & $0.172 \mathrm{~m}$ & $0.029 \mathrm{~m}$ \\
\hline \multirow{3}{*}{ PAM 2 } & Deflated $0 \mathrm{kPa}$ & $0.225 \mathrm{~m}$ & $0.02 \mathrm{~m}$ \\
& Inflated $138 \mathrm{kPa}$ & $0.171 \mathrm{~m}$ & $0.031 \mathrm{~m}$ \\
& Inflated $207 \mathrm{kPa}$ & $0.167 \mathrm{~m}$ & $0.033 \mathrm{~m}$ \\
& Inflated $276 \mathrm{kPa}$ & $0.165 \mathrm{~m}$ & $0.033 \mathrm{~m}$ \\
\hline
\end{tabular}


Table 3.5: Elongation of test muscle during cyclical test

\begin{tabular}{lccc}
\hline Pressure & Max Contraction & Percentage & Contraction \\
\hline \hline $0 \mathrm{kPa}$ & $0 \mathrm{~m}$ & $95 \%$ & $0 \mathrm{~m}$ \\
$35 \mathrm{kPa}$ & $0.038 \mathrm{~m}$ & $95 \%$ & $0.0361 \mathrm{~m}$ \\
$48 \mathrm{kPa}$ & $0.042 \mathrm{~m}$ & $95 \%$ & $0.0399 \mathrm{~m}$ \\
$55 \mathrm{kPa}$ & $0.043 \mathrm{~m}$ & $95 \%$ & $0.0409 \mathrm{~m}$ \\
$69 \mathrm{kPa}$ & $0.046 \mathrm{~m}$ & $95 \%$ & $0.0437 \mathrm{~m}$ \\
$83 \mathrm{kPa}$ & $0.049 \mathrm{~m}$ & $95 \%$ & $0.0476 \mathrm{~m}$ \\
$104 \mathrm{kPa}$ & $0.051 \mathrm{~m}$ & $95 \%$ & $0.0485 \mathrm{~m}$ \\
\hline & & $80 \%$ & $0 \mathrm{~m}$ \\
$0 \mathrm{kPa}$ & $0 \mathrm{~m}$ & $90 \%$ & $0 \mathrm{~m}$ \\
& & $95 \%$ & $0 \mathrm{~m}$ \\
\hline & & $80 \%$ & $0.043 \mathrm{~m}$ \\
$138 \mathrm{kPa}$ & $0.054 \mathrm{~m}$ & $90 \%$ & $0.048 \mathrm{~m}$ \\
& & $95 \%$ & $0.051 \mathrm{~m}$ \\
\hline $276 \mathrm{kPa}$ & $0.060 \mathrm{~m}$ & $90 \%$ & $0.054 \mathrm{~m}$ \\
& & $95 \%$ & $0.057 \mathrm{~m}$ \\
\hline
\end{tabular}

Both the PAM with and without the temperature sensor were tested in the Instron Universal testing machine as can be seen in Figure 3.11 


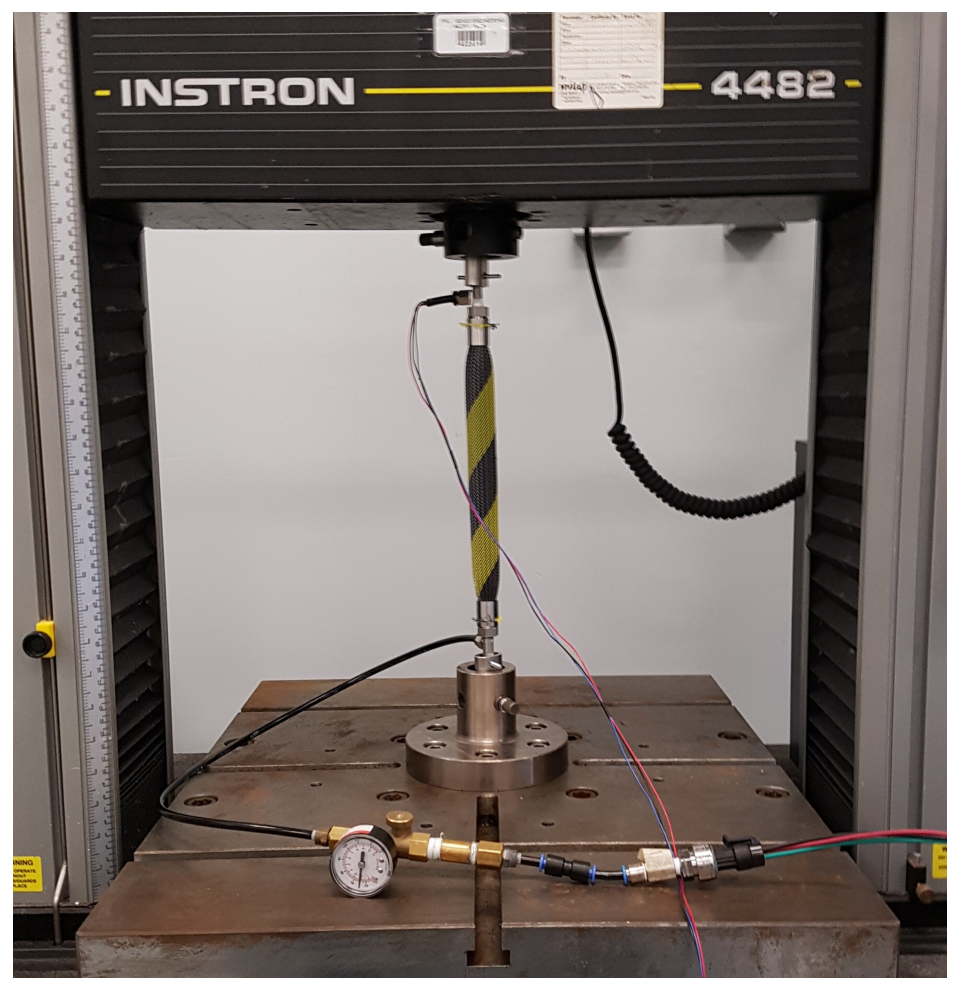

Figure 3.11: PAM testing setup in Instron linear testing machine. The wires feeding into the muscle are connected to the temperature sensor. The pressure transducer is also visible at the bottom of the photograph.

The testing began with the inflation of the muscle using a small air compressor. The pressure was monitored using the analog gauge integrated into the compressor. The value was then checked using the transducer. If the pressure was too high the valve was carefully opened to allow for a slight escape of gas. When the pressure was correct, the PAM was placed into the Instron machine and held using simple steel pins. The cross head of the tensile testing machine was slowly raised to remove the slack from the pin connections. The Instron was programmed to perform 10 cycles at a speed of $2.5 \mathrm{~mm} / \mathrm{s}$. Before the testing began, a large plexi-glass sheet was placed in front of the machine to protect the operator from flying debris in case of PAM rupture. The Instron machine records the displacement of the cross head and the force exerted on the load cell. A laptop computer was set up nearby and was connected to the Arduino Mega to record the pressure and the temperature inside the muscle. The synchronization of the two data sets was done in post processing based on the peaks of force and pressure. 


\subsubsection{Model Validation}

\section{Pressure and Force validation at high pressure}

To validate the model presented in Section 3.1, the muscle force, pressure and temperature were measured experimentally and compared to the simulated results.

The simulation outputs were read into a custom Matlab script so that they could be compared to the experimentally obtained data. The simulated results were plotted for three polytropic cases, the adiabatic case when $n g=1.4$, the isothermal case when $n g=1$, and one value in between when $n g=1.2$. Furthermore the RMSE values as well at the difference in amplitude of the peaks were calculated, these can be found in Appendix C

Figures 3.12 and 3.13 show the superposition of the experimental and simulated results for the rise in pressure inside the PAM. In both cases the PAM was initially inflated to $207 \mathrm{kPa}$, Figures 3.13 and 3.12 present the simulation with and without the bladder stress model respectively. There is little if any variation in the internal gas pressure of the PAM related to the presence or absence of the bladder stress model. This observation is true across all of the high pressure tests, the graphs of the other simulations can be found in Appendix C.2. Furthermore, the pressure variation is almost identical to the simulated isothermal case when $n g=1$. This indicates that the simulation in which the temperature of the PAM gas was kept constant is the best approximation when predicting the pressure variation in the high pressure tests. 


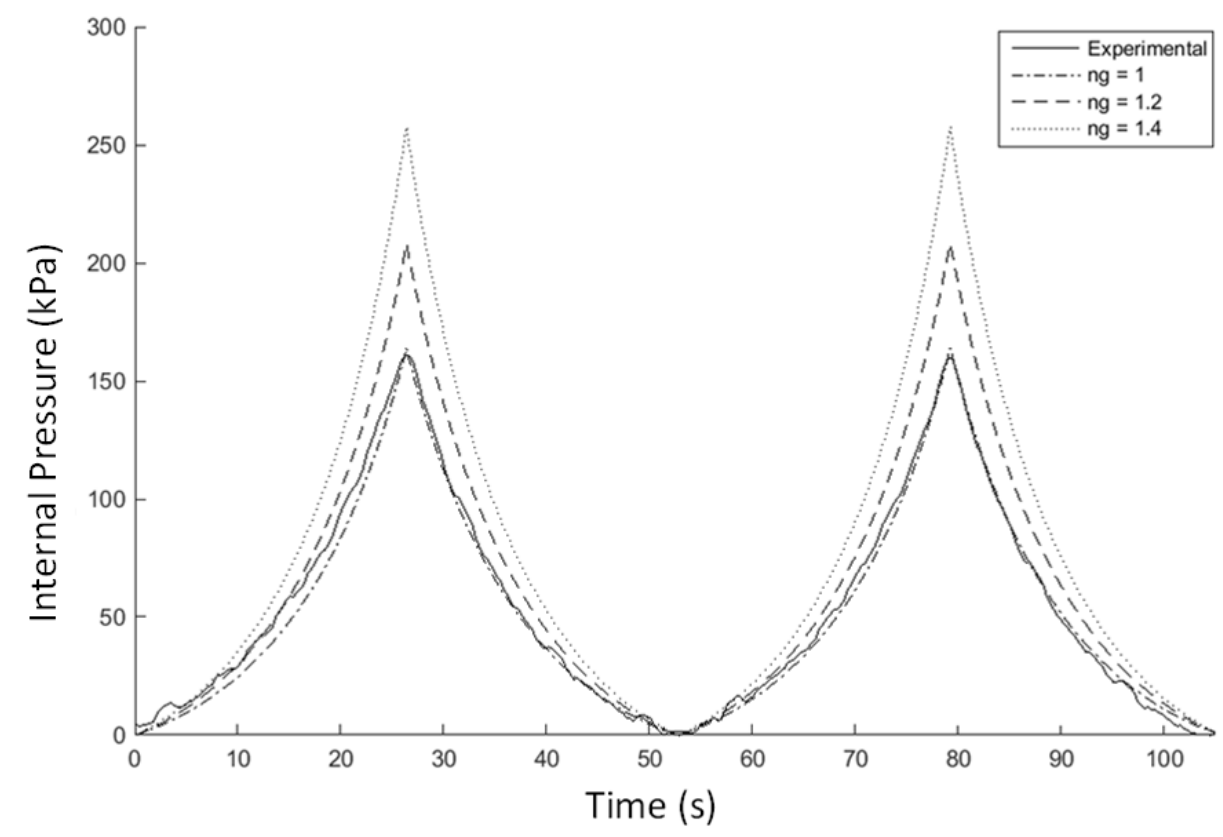

Figure 3.12: Comparison of the pressure results for the experimental and simulation without bladder stress model. The muscle was inflated to $207 \mathrm{kPa}$ and tested at an elongation of $90 \%$.

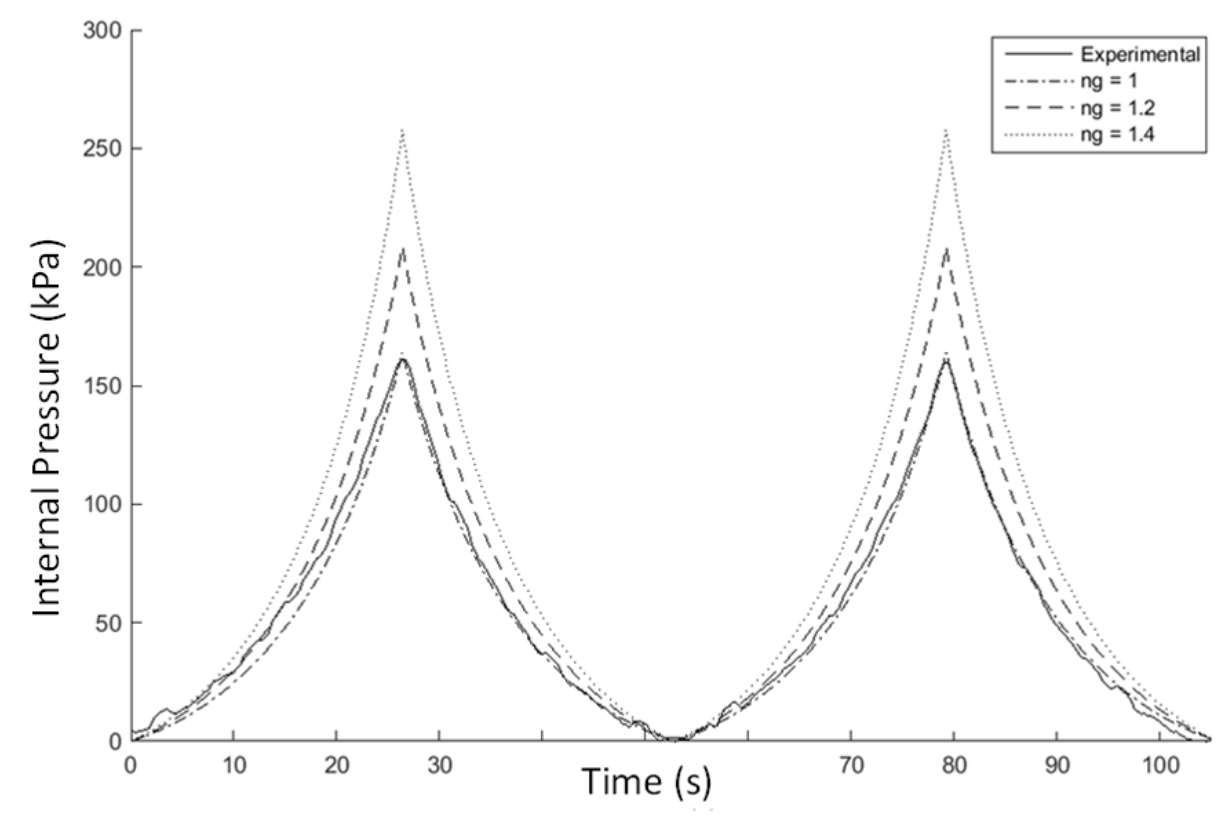

Figure 3.13: Comparison of the pressure results for the experimental and simulation with bladder stress. The muscle was inflated to $207 \mathrm{kPa}$ and tested at an elongation of $90 \%$.

The force generated by the muscle was also examined by superimposing the ex- 
perimental results and the simulated results. Figures 3.14 and 3.15 present the comparison of the simulations with and without the bladder stress model when inflated to $276 \mathrm{kPa}$.

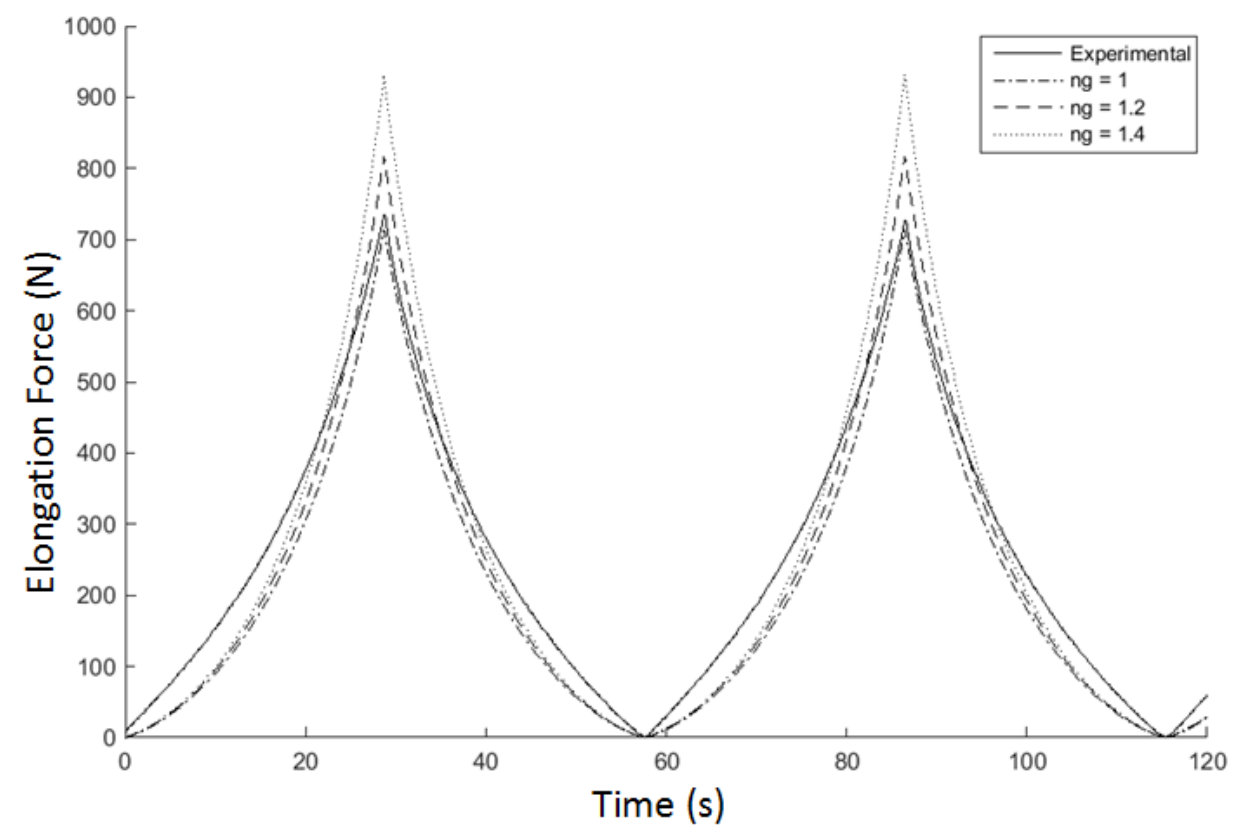

Figure 3.14: Comparison of the force results for the experimental and simulation with bladder stress, at $276 \mathrm{kPa}$ at elongation of $95 \%$.

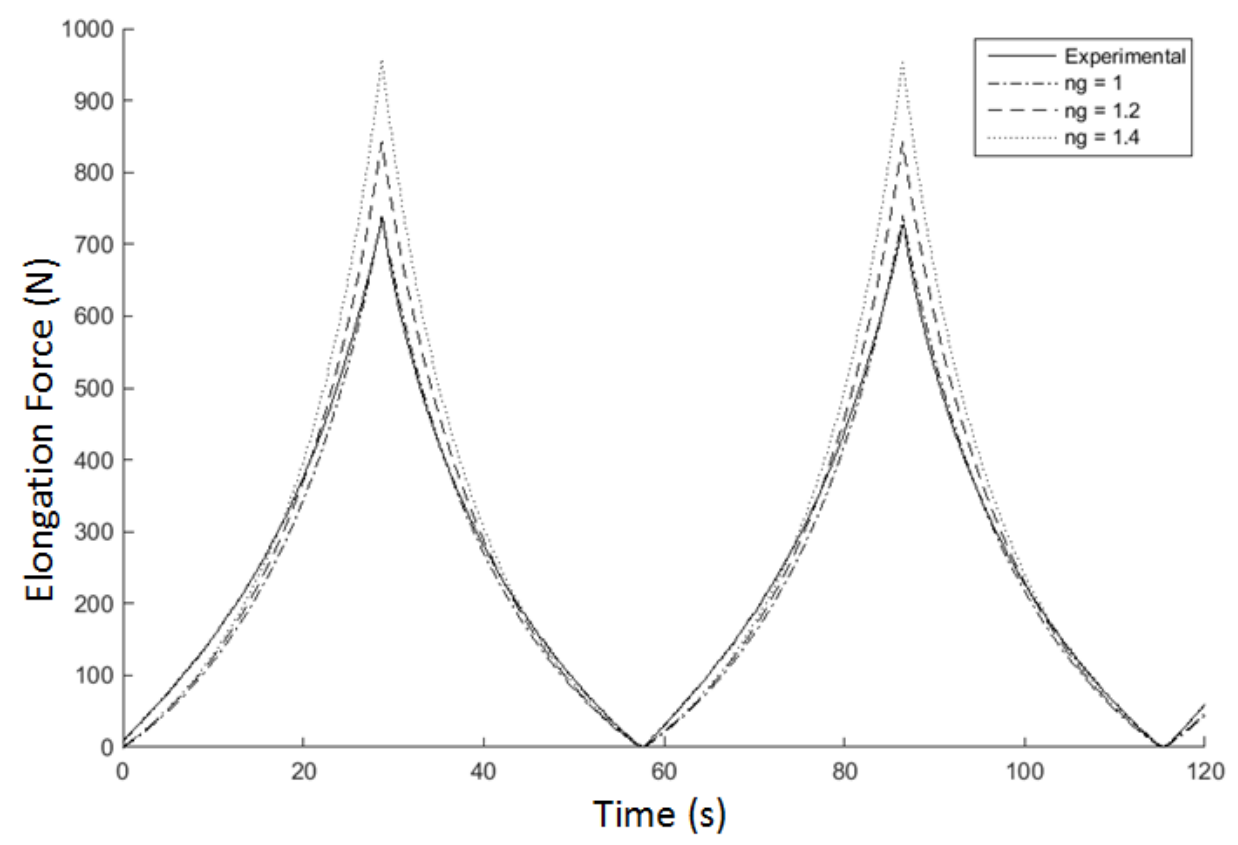

Figure 3.15: Comparison of the force results for the experimental and simulation without bladder stress model, at $276 \mathrm{kPa}$ at elongation of $95 \%$. 
The addition of the bladder stress model decreased the force predicted by the simulation slightly, see Table C.7. According to the RMSE tables found in Appendix C, the inclusion of the bladder stress model increased the error slightly. This is illustrated by the slight change in shape of the graphs. It is unclear what may be the cause of this difference. However, the force generated by the PAM is highly dependent upon the friction. Moreover, the friction in the PAM is very complex and challenging to characterize since it depends on multiple subtle features such as the interaction of the bladder and the mesh. For instance a very soft bladder material will tend to deform around the mesh. This indentation of the fibers fills in the gaps in the mesh and generates an additional parameter that may induce friction. In addition, this phenomenon is directly affected by the braid angle, tightness of the weave as well as the internal gas pressure. The indentation of the fibers into the bladder was not examined in this model.

The force results in this section illustrate that the maximum force produced varies based on the polytropic index as well as the presence of the bladder stress model. The variation caused by the latter being almost negligible.

\section{Pressure and Force validation at low pressure}

The simulation was achieved for the low pressure cases using the appropriate settings and geometric measurements. For these tests, values such as the diameter and length of the muscle were specific to each test case, all other values, such as friction coefficients, remained unchanged. Figures 3.17 and 3.16 present the comparison of the force for a PAM inflated to $69 \mathrm{kPa}$ when compared to the simulation with and without the bladder stress model. 


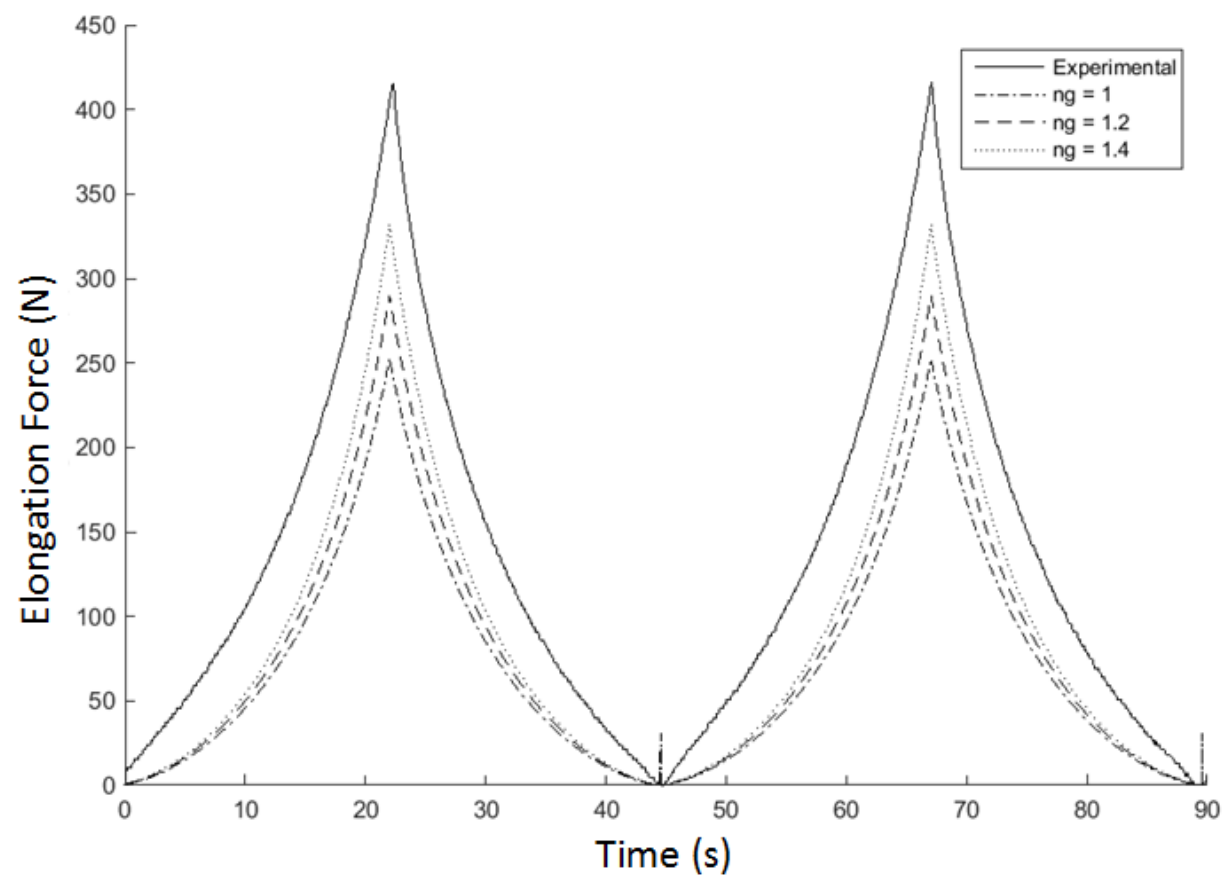

Figure 3.16: Comparison of the force results for the experimental and simulation with bladder stress model included, at $69 \mathrm{kPa}$.

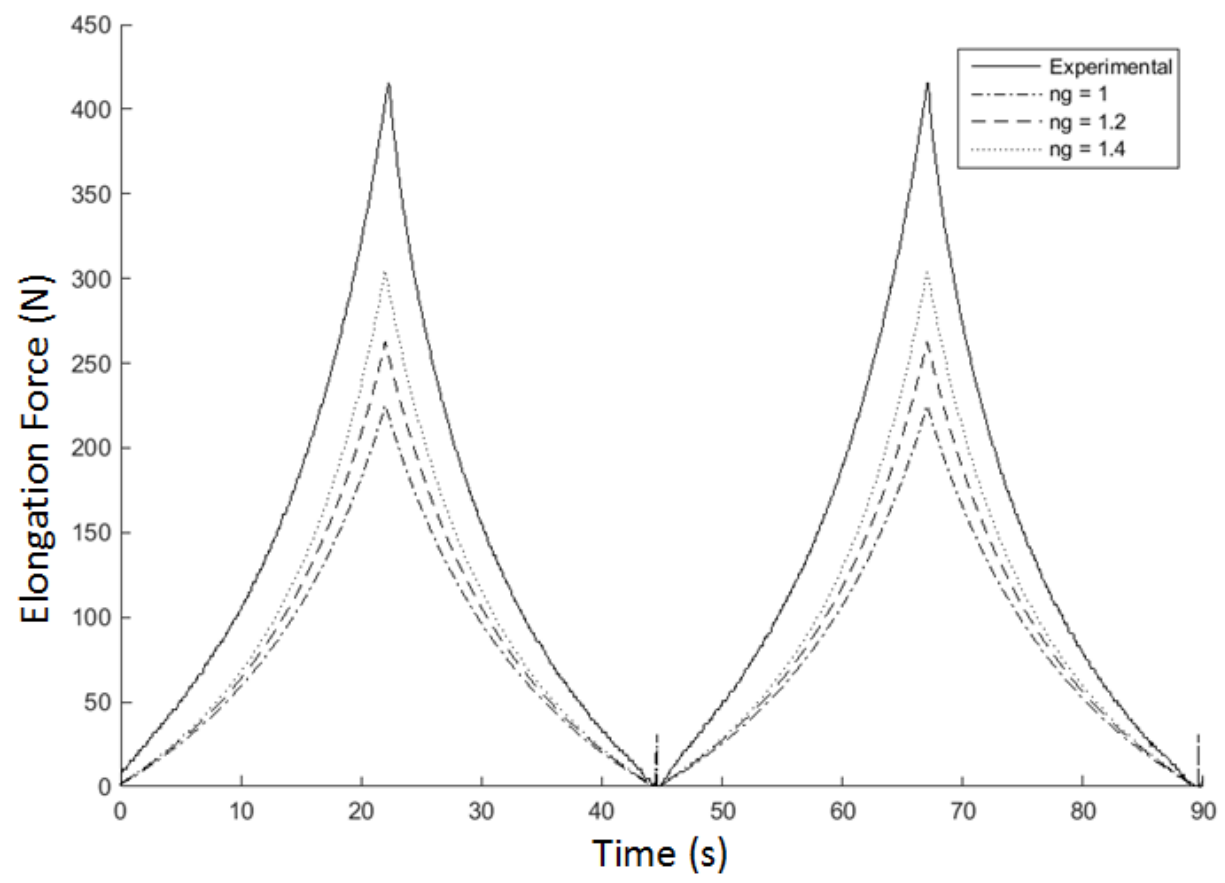

Figure 3.17: Comparison of the force results for the experimental and simulation without bladder stress model, at $69 \mathrm{kPa}$.

With reference to Figures 3.17 and 3.16, the experimental values of force are well 
above the simulated values. This is a known limitation of the model at low pressure. Furthermore, the addition of the bladder stress model increased the predicted force muscle force and brought the simulated force values closer to the experimentally obtained values. For example, 3.17 shows the force for a PAM tested at $69 \mathrm{kPa}$ compared with the simulation run without the bladder stress model. The graph shows that the experimental force is more than $100 \mathrm{~N}$ above the maximum force predicted by the simulation. However when the bladder stress model is included the maximum force magnitudes are closer, see Figure 3.16

The pressure results were also examined. Figures 3.18 and 3.19 present the comparison of the simulated and experimental pressure data for a PAM inflated to $55 \mathrm{kPa}$ with and without the inclusion of the bladder stress model.

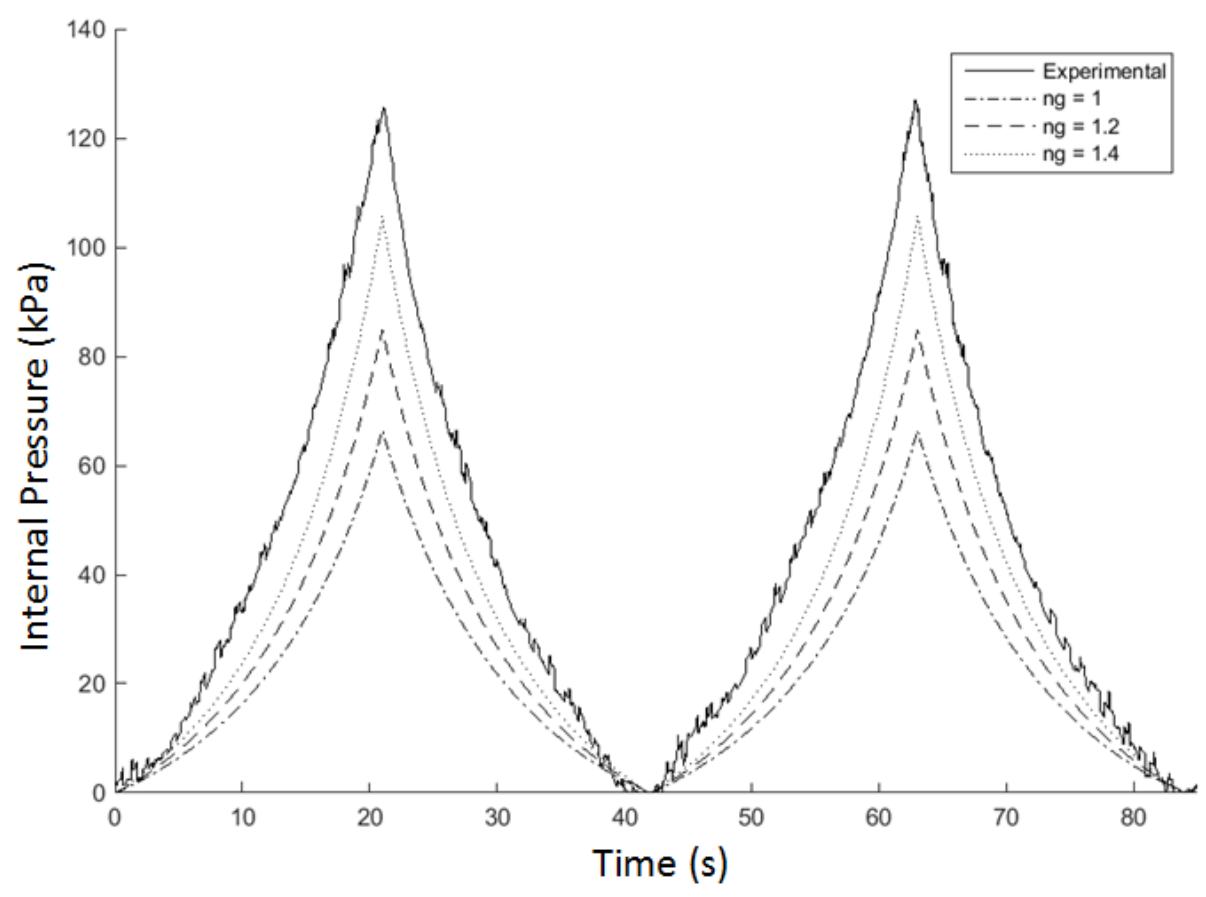

Figure 3.18: Comparison of the pressure results for the experimental and simulation with bladder stress, at $55 \mathrm{kPa}$. 


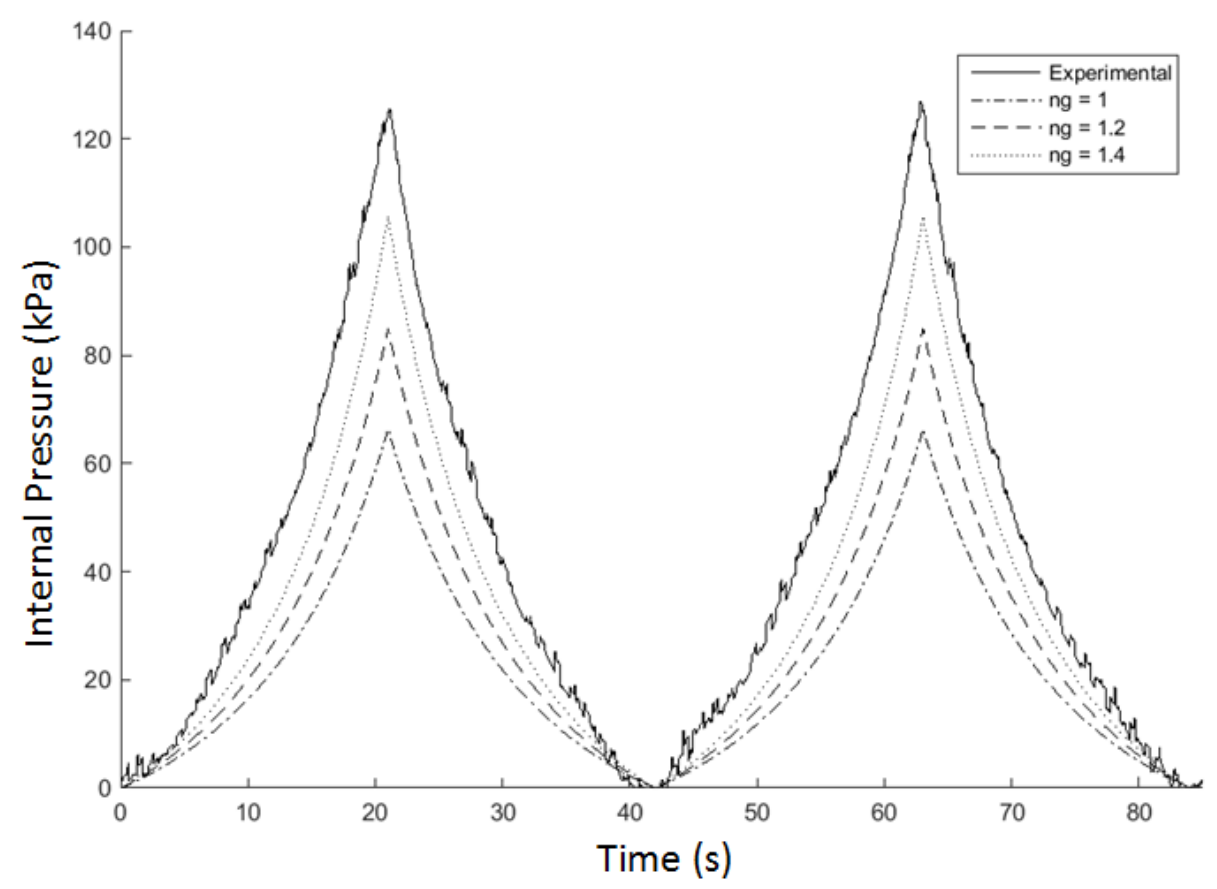

Figure 3.19: Comparison of the pressure results for the experimental and simulation without bladder stress model, at $55 \mathrm{kPa}$.

With reference to Figures 3.18 and 3.19, the experimental values are well above the simulated values. This is similar to the force results. However, the presence of the bladder stress model does not affect the pressure the same way as it affects the force. From the force results, the bladder stress model brought the simulated results closer to the experimental values, see Table C.5. The same cannot be said for the pressure. The pressure outputted by the simulation with and without the bladder stress model, shows little if any difference. This was expected since the bladder stress model should theoretically only affect the force, furthermore, this is consistent with the high pressure tests.

\section{Temperature validation}

The internal temperature of the muscle was recorded during the high pressure tests. Figure 3.20 presents the temperature variation of the gas inside the PAM. The Figure presents the PAM inflated to $207 \mathrm{kPa}$ and $276 \mathrm{kPa}$, for both pressures elongations of $80 \% 90 \%$ and $95 \%$ are shown. The results presented in Figure 3.20 are summarized in Table 3.6. 

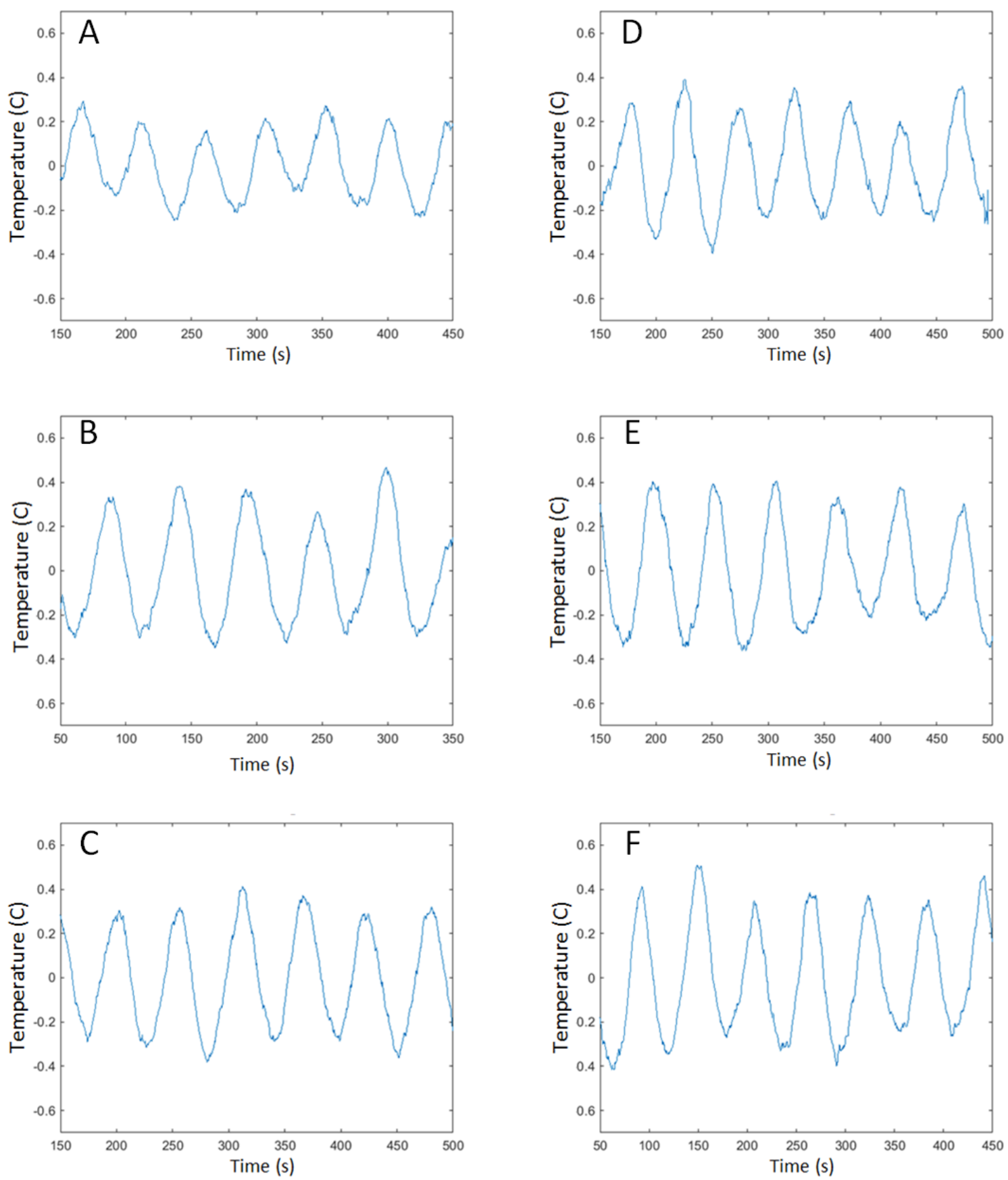

Figure 3.20: Internal gas temperature for cyclical testing elongations of $80 \% 90 \%$ and $95 \%$ for pressure of $207 \mathrm{kPa}(\mathrm{A}, \mathrm{B}, \mathrm{C})$, and $276 \mathrm{kPa}(\mathrm{D}, \mathrm{E}, \mathrm{F})$ 
Table 3.6: Temperature fluctuation of gas inside PAM

\begin{tabular}{ccc}
\hline Elongation percentage & Gas pressure $207 \mathrm{kPa}$ & Gas pressure $276 \mathrm{kPa}$ \\
\hline \hline $80 \%$ & $0.4^{\circ} \mathrm{C}$ & $0.5^{\circ} \mathrm{C}$ \\
$90 \%$ & $0.7^{\circ} \mathrm{C}$ & $0.7^{\circ} \mathrm{C}$ \\
$95 \%$ & $0.7^{\circ} \mathrm{C}$ & $0.8^{\circ} \mathrm{C}$ \\
\hline
\end{tabular}

As the muscle is stretched, the volume decreases increasing the gas pressure and theoretically the temperature as well. Figure 3.20 and Table 3.6 present the variation of the gas temperature. The maximum temperature variation was $0.8^{\circ} \mathrm{C}$. Figure 3.21 presents the comparison of the experimental and simulated temperature results.

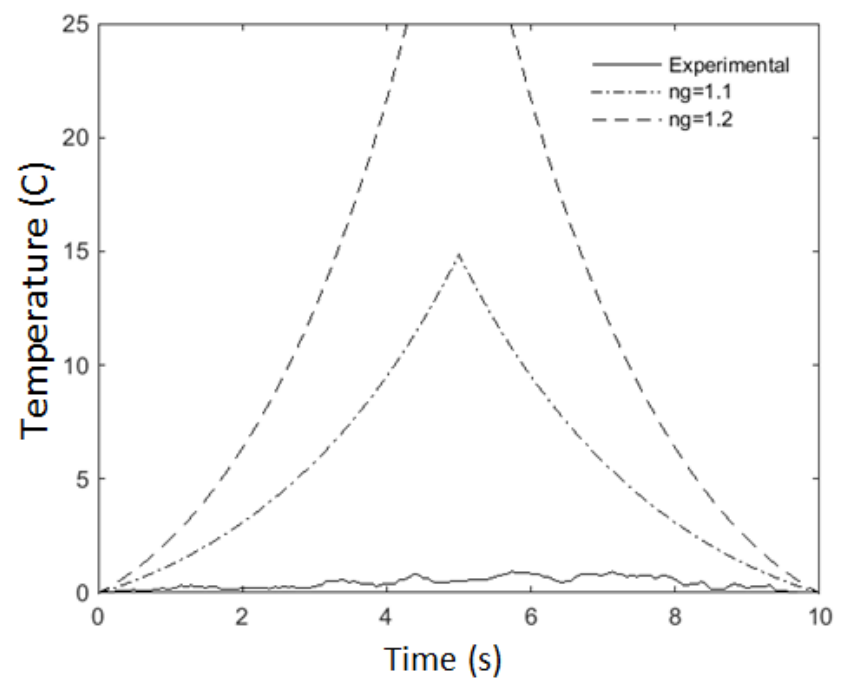

Figure 3.21: Comparison of the rise of the internal temperature predicted by the simulation and the measured temperature for the $276 \mathrm{kPa} 95 \%$ elongation scenario.

Figure 3.21 clearly demonstrates that the measured temperature fluctuation of less than $1^{\circ} \mathrm{C}$ is well below the simulated values. The measured temperature is much closer to the isothermal condition than the adiabatic condition.

\section{Conclusion}

This chapter presented PAM model including a polytropic gas approach that did not assume the compression to be isothermal, as well as a model to characterize 
the deformation of the bladder material.

Simulations were carried out to predict the changes in muscle force, pressure, and temperature during passive elongation. This simulation was validated with experimental testing. The force and pressure results showed that at higher pressures, the PAM matched the isothermal case very well. However, at low pressure the simulated values underestimated the experimental values. At these low pressures, the addition of the bladder stress model improved the performance of the force simulation but did not affect the pressure variation. The temperature measurements revealed a variation of less than $1{ }^{\circ} \mathrm{C}$ during the high pressure elongation cycle. This indicates that the isothermal case is most appropriate. This is further supported by the pressure values obtained from the high pressure tests which also fit the isothermal case best. Therefore, the previously made assumptions of an isothermal gas process is very near to the measured temperature variation and is thus a fairly accurate assumption. 


\section{Chapter 4: Design, Modelling, Fabrication and Mechanical Testing}

This chapter presents the design, modelling fabrication and mechanical testing of the proposed ankle exoskeleton. The design process started by defining the design criteria then a conceptual design was proposed. A Matlab simulation was used to examine the behaviour of the design and compare it to the behaviour of the natural ankle.

To accelerate the iterative prototyping process, 3D printing was used to fabricate a prototype for preliminary testing. The final prototype was constructed at the University of Ottawa Machine Shop.

In order to evaluate the fabricated prototype, experimental testing was done using the Instron universal testing machine. The goal of the testing was to confirm that the device is able to produce an ankle torque profile similar in magnitude and shape to the human ankle. The detailed testing procedure and results are presented in this chapter. 


\subsection{Design Parameters}

The proposed ankle exoskeleton is intended to assist the user by enhancing the moment about the ankle joint without altering the gait of the individual. Thus the weight, adaptability and level of assistance were carefully considered. Moreover, the device must not interfere with the natural motion of the joint. Therefore, the device must allow $50^{\circ}$ of plantarflexion and $20^{\circ}$ of dorsiflexion. These are average values of the maximum range of motion of the human ankle joint in the sagittal plane, as shown in Table A.1.

Section A.1.5 in Appendix A, demonstrated the energetic consequences of added mass on the legs during walking. To avoid additional mass, the proposed device will be light and passively powered. The device will not use any exterior power for force production or actuation. This will allow the device to be kept low profile and very light so that the torque provided will assist the user and not only offset its own weight. The design requirements were guided by certain restrictions that must be met and multiple criteria that outline desirable traits that were not mandatory. These are summarized in Table 4.1

The proposed exoskeleton generates an assistive torque during plantarflexion; however, during the gait cycle the ankle goes through this motion twice. Once when the foot is on the ground and once while the foot is in swing phase. It would be problematic if the user had to fight the device during the swing phase. Therefore, a clutch mechanism that engages and disengage the spring was required. Furthermore, in order to adapt the device to different users, the timing of the engagement mechanism must be tunable. 
Table 4.1: Summary of design restrictions and other desirable criteria

Passively powered

Achieve $20^{\circ}$ dorsiflexion and $50^{\circ}$ plantarflexion

Restrictions Weigh $\leq 4 \mathrm{~kg}$

Generate $25 \%$ of natural plantarflexion torque

Disengage during swing phase

Adjustable for different users

Criteria

Be as comfortable as possible

Be as light as possible

Allow normal gait

\subsection{Proposed design}

The final prototype is presented in Figure 4.1 


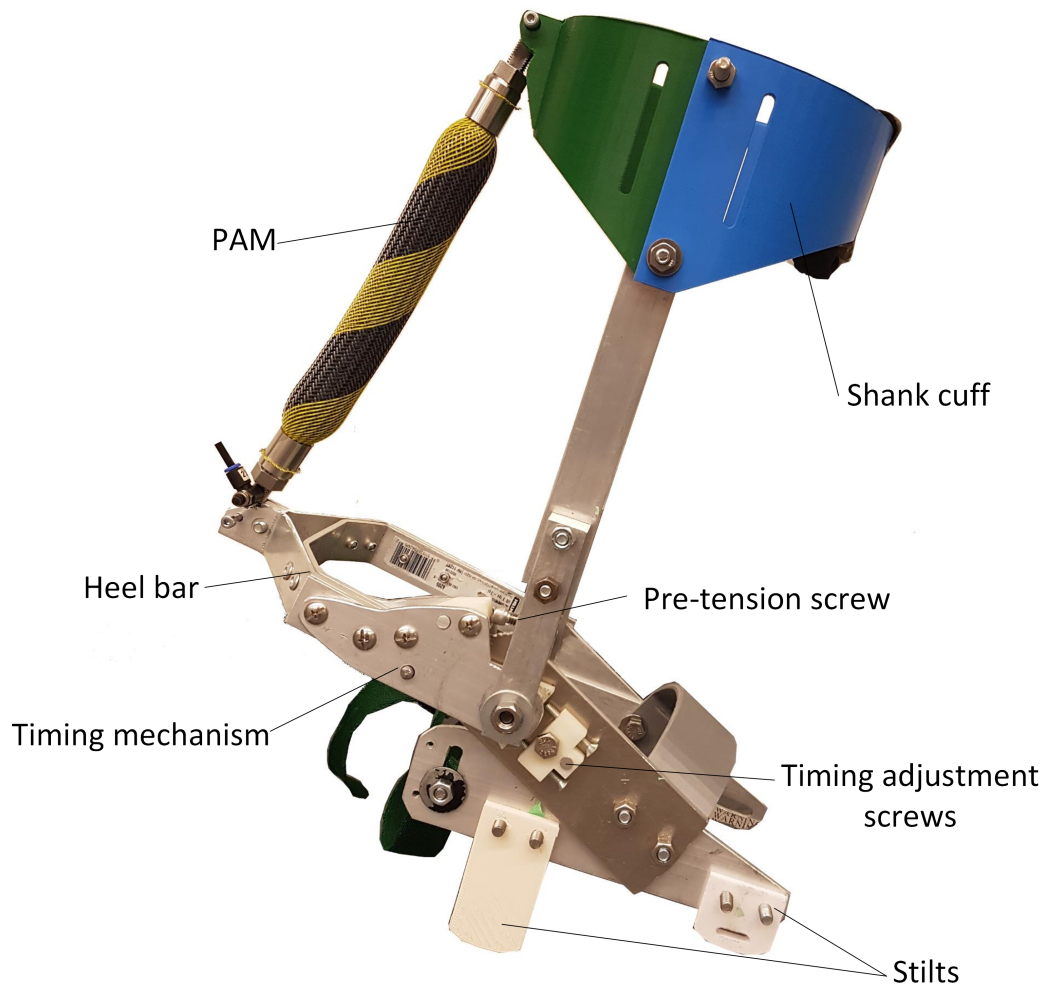

Figure 4.1: Photograph of the completed exoskeleton prototype

Based on the design criteria and restrictions, several conceptual designs were conceived for analysis and evaluation. All designs are based on the function of the natural Achilles tendon. Therefore, a spring element and dashpot element were designed in parallel with the lower leg using proximal and distal anchor points. In order to generate an optimum ankle torque, a pivoting joint aligned with the ankle was required. Furthermore, the torque production also requires a moment arm that extended past the heel, out to the distal spring attachment point. The second, proximal, attachment point is attached to a cuff just below the knee. This cuff needed to be rigidly connected to the ankle joint. This ensured the intended transfer of force. Without a rigid connection between the ankle joint and the upper cuff, the downward force generated by the spring would create large shear forces that would tend to slide the cuff down the leg. Not only would this create an energy loss inside the system, it would also be very uncomfortable for the user.

The device has a cuff around the calf just below the knee (shank cuff). A slender rod extends down the side of the leg to the ankle joint. At this joint, a rod extends forward along the length of the foot and a second member extends backwards past the heel to connect to the distal end of the spring element (heel bar). This was mirrored 
on both sides of the leg. The timing mechanism is located just behind the ankle joint on one side of the foot. Two small plastic stilts maintain joint alignment by preventing the mechanical joint from sliding down towards the ground.

\subsection{Kinematic Simulation}

With the general structure of the device determined, a simplified 2D kinematic analysis was conducted. This was done to quantify the function of the device throughout the gait cycle prior to prototype fabrication. Written in Matlab, the simulation used anthropometric data obtained through experimental walking trials [55]. The code can be found in Appendix G. The given angles of the hip knee and ankle joints moved the simulated lower limbs through the normal walking cycle. The dimensions of the hypothetical exoskeleton were superimposed onto the simulated lower limbs, see figure 4.2. This simulation was made to find a combination of device dimensions and spring characteristics that together produce an ankle joint torque profile similar to that of the natural ankle joint during walking.

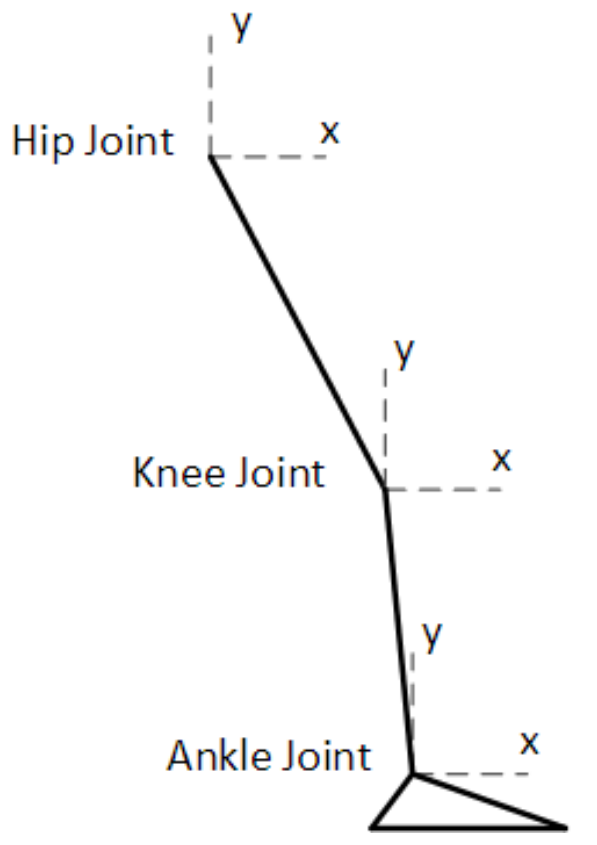

Knee Joint

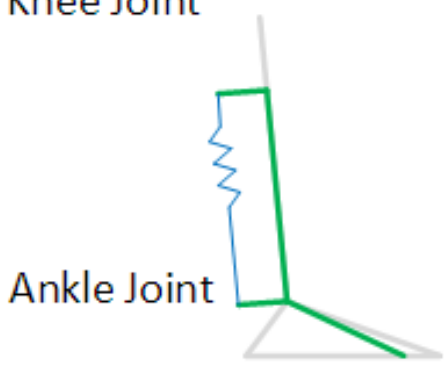

Figure 4.2: Simplified layout of the lower limbs used during kinematic simulations.

In the human ankle, plantarflexion is the result of the calf muscles generating a tension force that is transferred to the Calcaneus bone via the Achilles tendon. During 
the gait cycle, the angle of the shank relative to the foot varies continuously. This principle is amplified in the exoskeleton device due to the extended moment arm. To demonstrate the variation of the effective moment arm throughout the stance phase, Figure 4.3 was made. It shows that the changing angle of the device had an effect on the perpendicular distance between the spring line of action and the center of rotation. Figure 4.4 shows the variation of the moment arm of the device during gait.

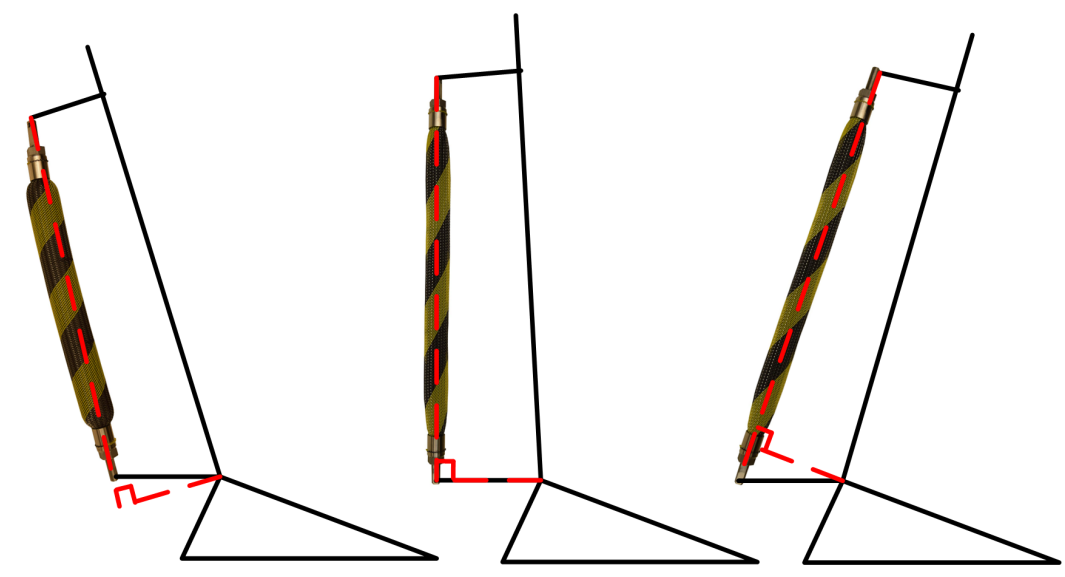

Figure 4.3: Illustration of the variation of the effective moment arm of the device during stance phase. The direction of the spring force relative to the ankle joint. The dotted line represents the perpendicular distance between the force and the rotation point. 


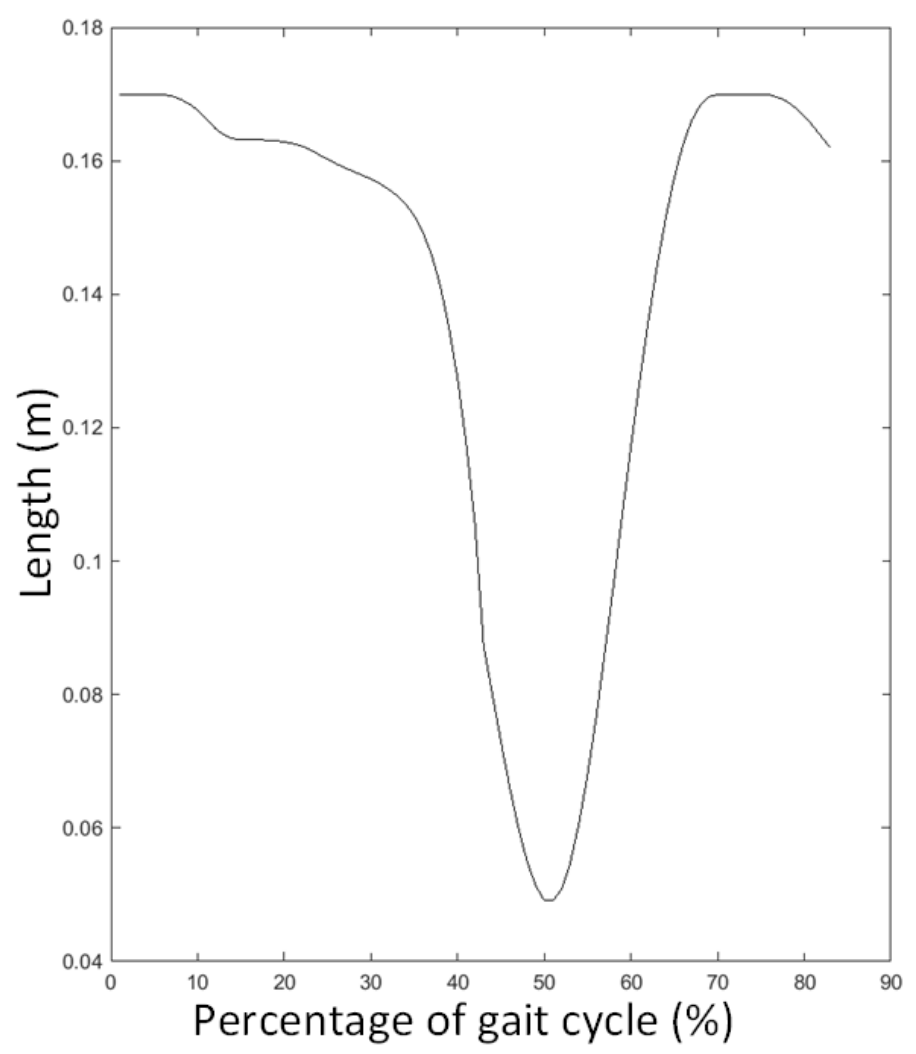

Figure 4.4: Illustration of the variation of the effective moment arm of the device. This is the perpendicular distance between the line of action of the force and the pivot point.

Figure 4.4 shows the large amplitude of variation of the moment arm length. Over a single gait cycle, the effective moment arm fluctuated between 5 and $17 \mathrm{~cm}$. The presence of a clutch mechanism disengages the spring during the swing phase. Thus, the resulting moment falls to zero regardless of the moment arm length. The next section will describe the clutch mechanism in more detail.

The tension force produced by the spring element, and therefore the ankle torque, is directly related to the elongation of the spring. The elongation of the spring element is shown in Figure 4.5. Figure 4.5 B) shows that the total elongation of the spring is $4.25 \mathrm{~cm}$. Furthermore, Figure 4.5 demonstrates that the elongation of the spring is confined to the portion of the gait during which the clutch mechanism is engaged. 

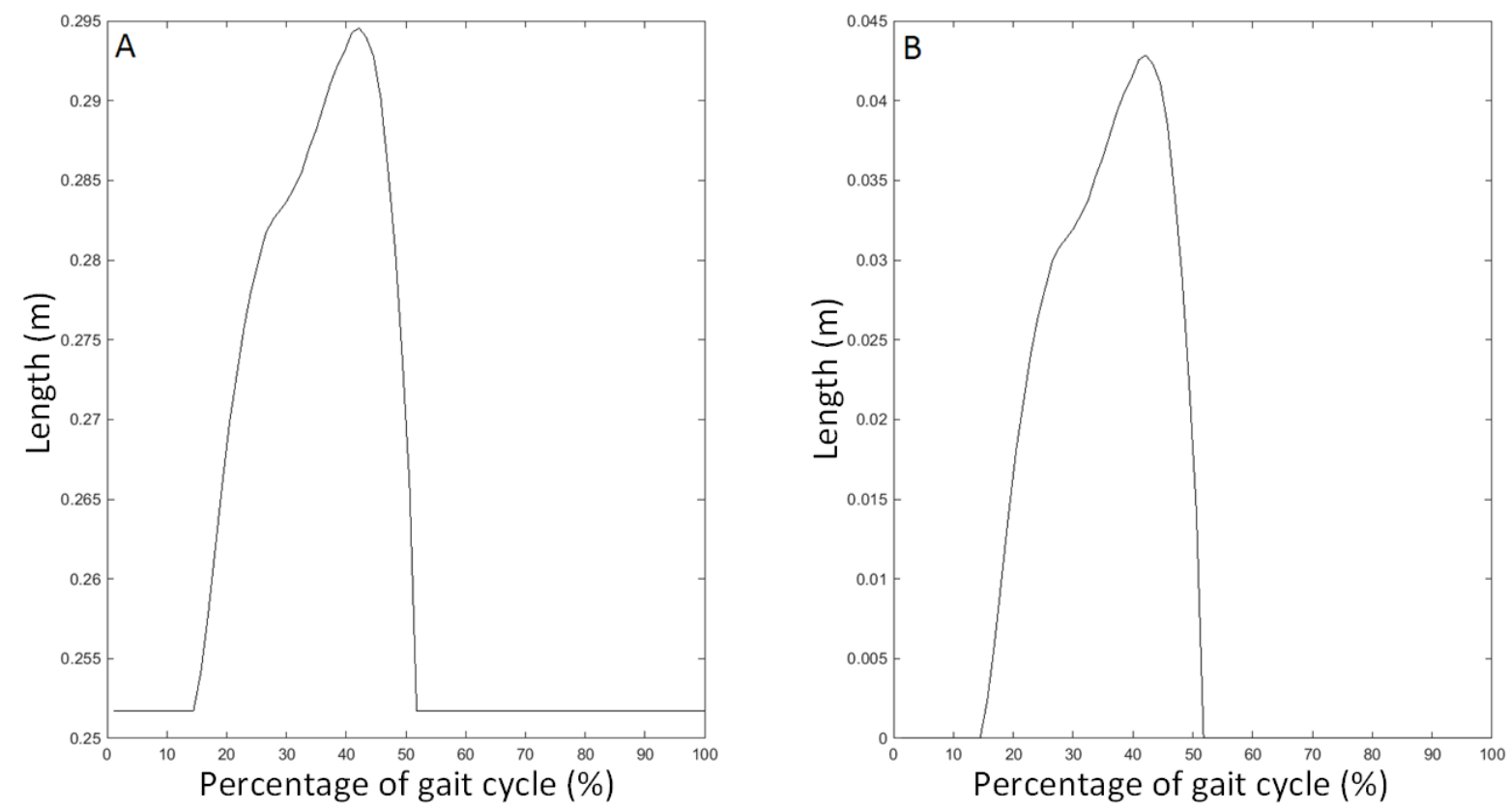

Figure 4.5: A) illustrates the length of the spring element during the gait cycle. The length of the spring varies approximately between 25 and $30 \mathrm{~cm}$. B) illustrates the net elongation of the spring during the gait cycle

With the moment arm and spring elongation both known, the force generated by the spring was determined. The force generated by the spring depends on its stiffness. In order to evaluate if a classical coil spring or a PAM was better suited for this application, they were compared in the simulation. A linear equation was used to predict a classic coil spring and a non-linear equation for the PAM. The PAM equation was obtained by fitting a fourth order polynomial to previous PAM mechanical testing data. For comparison, the ankle torque profile for the device using a linear spring, the device using a PAM and the natural ankle torque can be seen Figure 4.6. The maximum magnitudes have been normalized to $25 \%$ of the natural torque. 


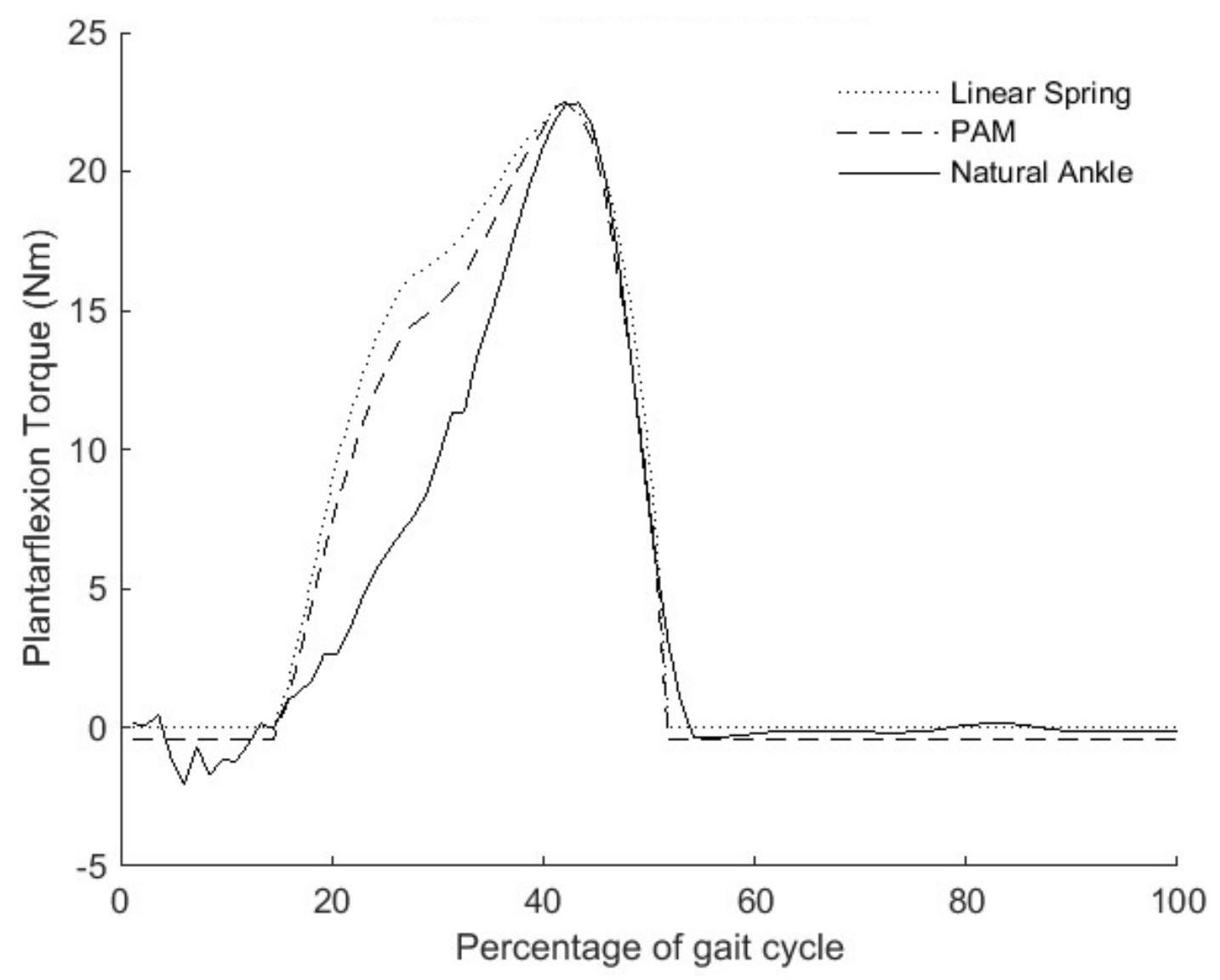

Figure 4.6: Comparison of torque profiles. Both linear and PAM springs are assumed to be used with the exoskeleton device. The magnitudes have been normalized to $25 \%$ of the maximum natural ankle torque

From Figure 4.6 it seems that neither the linear spring nor the PAM spring replicate the torque profile of the natural ankle joint during walking. However it appears that the PAM is a better fit than the linear spring. Moreover, the non linear PAM equation used for this simulation was obtained from one particular test. By varying parameters such as the PAM length, pressure, braid type and bladder material, the stiffness can be fine-tuned to match the stiffness of human soft tissue.

To further examine the torque profile that the proposed device could produce, the calculations were executed in reverse. The natural torque profile is taken as the input and used to generate the characteristics of the ideal spring. This so called ideal spring, is the hypothetical spring that should be used with this particular device in order to perfectly replicate the natural torque profile. As shown in figure 4.7 the force vs length relationship of this hypothetical spring element is not linear. Rather, the graph shows an increasing stiffness. This profile is very similar to the stiffness of the 
PAM, Figure 4.8.

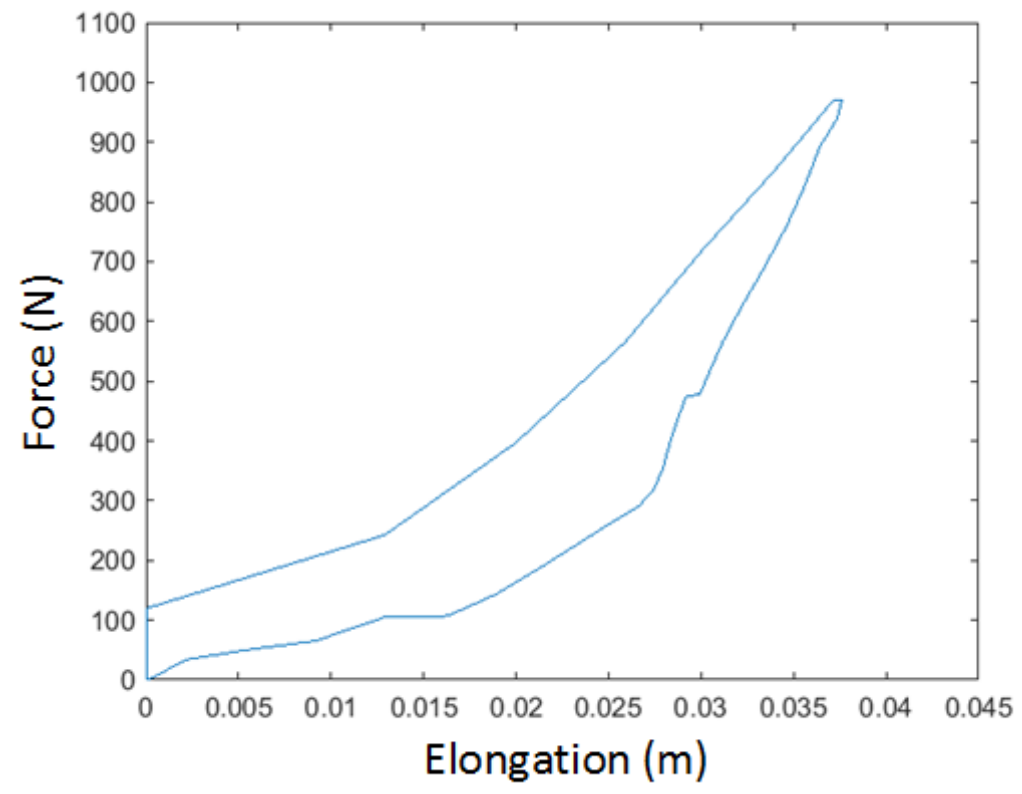

Figure 4.7: Force vs Elongation profile of the theoretically ideal spring if the device were to replicate the normal torque profile of the natural ankle joint for a person weighing $86 \mathrm{~kg}$

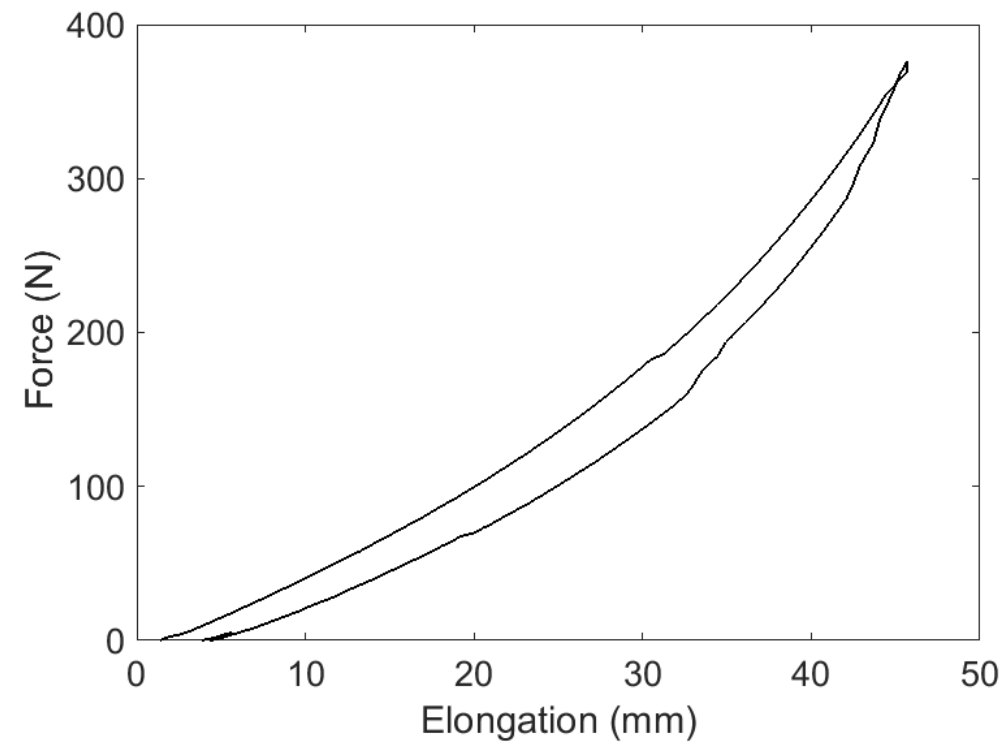

Figure 4.8: Force vs Elongation profile of a PAM with 2 fiber revolutions and an initial pressure of $138 \mathrm{kPa}$

According to this simulation, a non linear spring is required to precisely match 
the biological case. Furthermore, the spring needs to be approximately $25 \mathrm{~cm}$ in length and be able to stretch up to $4 \mathrm{~cm}$. The experimental testing results presented in Chapter 3 indicated that the muscles with 2 fiber revolutions were able to stretch up to $5 \mathrm{~cm}$. Furthermore, if the end caps are included, the length of the PAM fits the requirements detailed in this section. Figure 4.7 shows that the ideal spring element should produce approximately $1000 \mathrm{~N}$ with and elongation of $4 \mathrm{~cm}$. The device is intended to produce $25 \%$ of the ankle torque, thus the spring element is required to produce around $250 \mathrm{~N}$. This is well within the range of forces produced by PAMs. Therefore, a PAM with 2 fiber revolutions is a good candidate because of its original length, maximum elongation distance and non linear stiffness.

\subsection{Timing Mechanism and operational phases}

The core principle behind the clutch mechanism is a ratchet gear. Figure 4.9 presents the basic mechanism. A two tier pawl interacts with both the ratchet gear as well as the engagement arm. The lower part of the pawl has teeth that mesh with the ratchet. The upper portion of the pawl acts like a cam surface. As the engagement arm slides along this upper surface, the pawl teeth are pushed away from the gear. When engaged, the ratchet gear is blocked from rotating in one direction, but it can rotate in the opposite direction. When disengaged the ratchet gear is able to rotate freely. 

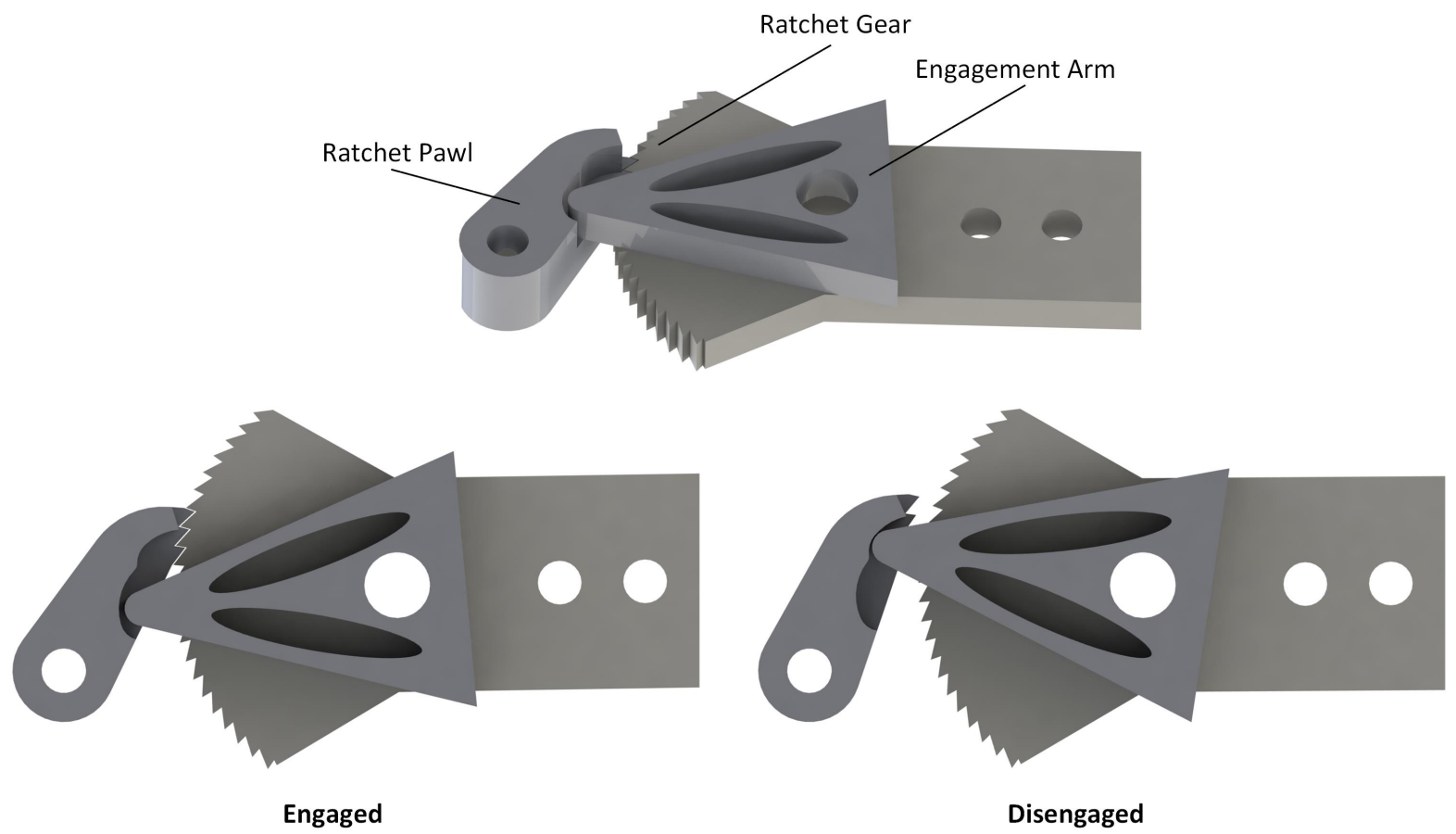

Figure 4.9: Illustration of the engagement and disengagement of the timing mechanism.

The timing of the engagement and disengagement is dictated by the position of the engagement arm. To adjust and customize this timing, two screws extend behind the engagement arm and limit its rotation, these screws can be seen in Figure 4.10.

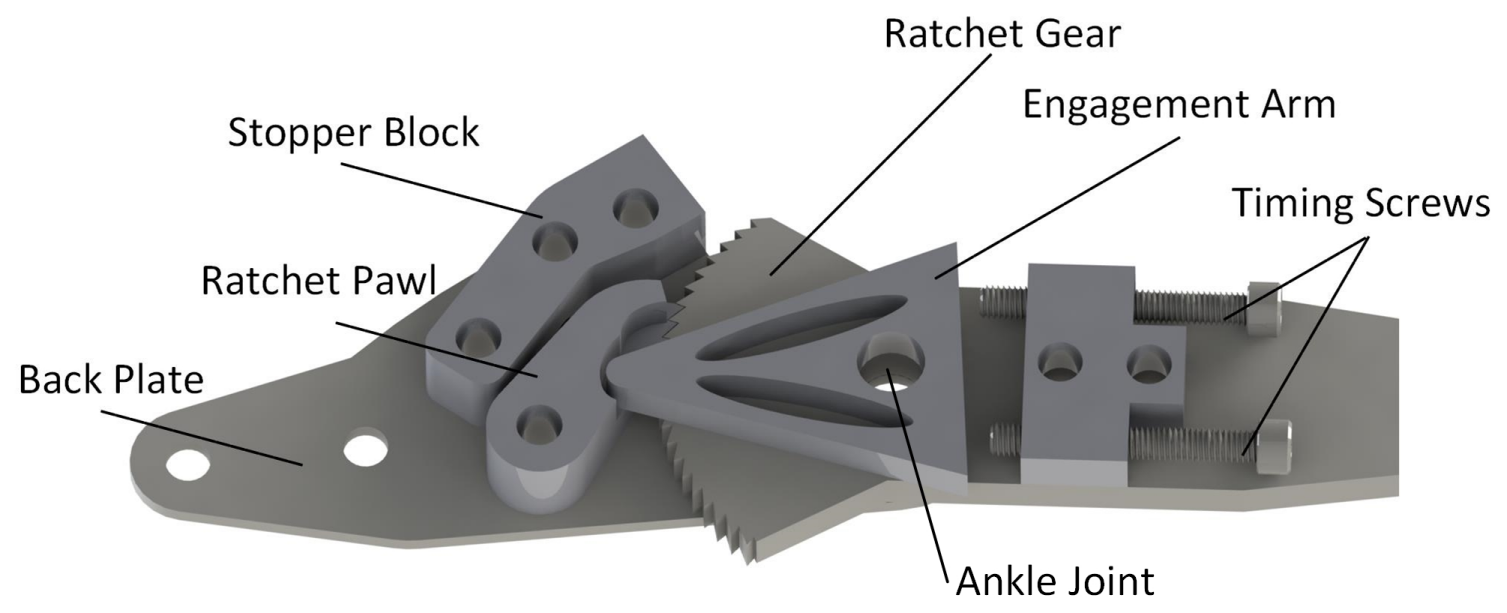

Figure 4.10: Illustration of the complete timing mechanism. The Back Plate, Ratchet Gear plate as well as the Engagement arm are all free to rotate independently around the Ankle joint 
The pawl is held against the gear by a spring. This spring is found between the pawl and the Stopper Block, it is not visible in Figure 4.10. The Stopper Block not only anchors the spring it also acts as a stop for the heel bar and can be used to pre-tension the spring. This can be seen in Figure 4.11. The figure illustrates the entire exoskeleton with a close up of the timing mechanism. The pre-tension screw is threaded through the shank upright. The end of the screw extends posteriorly towards the heel bar. This screw is a physical obstacle that prevents the stopper block from rising. The adjustment of this screw sets the minimum length of the spring. Thus, this screw can be used to either pre-tension the spring or to adjust for the length of different spring elements.

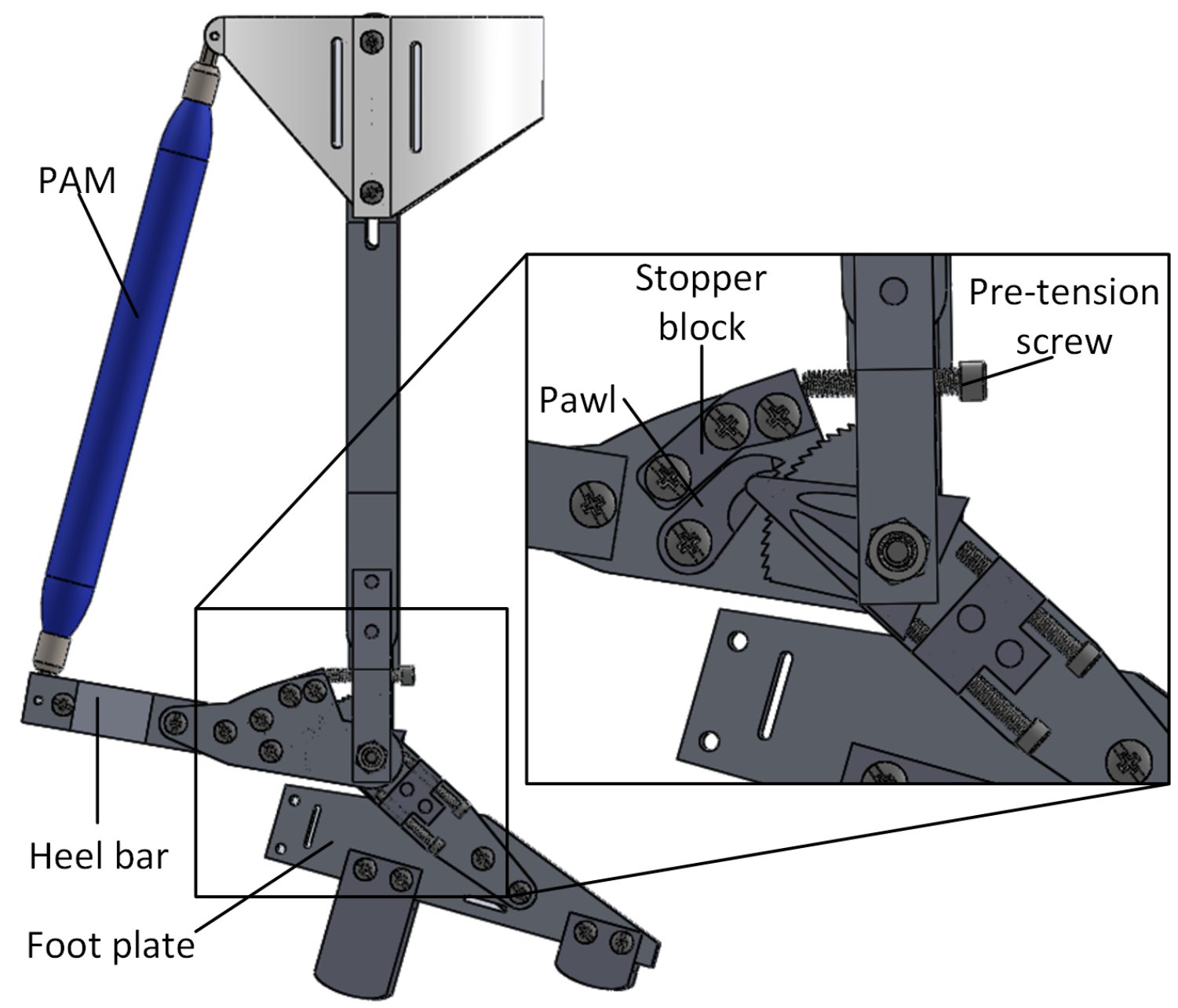

Figure 4.11: Illustration of the entire exoskeleton model showing the position of the timing mechanism as well as the pre-tension screw.

The function of the timing mechanism is illustrated in Figure 4.12. When the 
foot first touches the ground the ankle dorsiflexes so that the heel contacts the ground first. This dorsiflexion angle causes the mechanism to move from the disengaged to the engaged state, see Figure 4.12 (A). This engagement process causes the ratchet gear to rotate counterclockwise and push the engagement arm, which in turn slides towards the bottom of the pawl and allows it to engage with the ratchet gear. In this position, the two sides of the mechanism and linked through the gear and pawl, forces acting on the heel bar are transferred to the foot plate and the user. This is the end of the engagement process. Just after the initial contact (and mechanism engagement), the front of the foot falls until it is flat on the ground. The ratchet mechanism allows this rotation since the pawl simply slips to the next gear tooth. Once the foot is flat on the ground, the ankle begins to dorsiflex as the body passes into the stance phase and the loading of the spring begins. The mechanism does not allow rotation in this direction since the heel bar is fixed in relation to the foot plate. This creates the elongation in the spring element. Next comes the push off phase. During this phase, the energy stored in the spring element is returned to the user in the form of a plantarflexion torque. As the knee breaks and the foot pushes off the ground, the ankle reaches a maximum plantarflexion angle. This angle triggers the disengagement process, $4.12(\mathrm{C})$. The engagement arm slides towards the tip of the pawl and push it away from the gear, thus disengaging it. The mechanism is now disengaged and the foot is free to rotate during the swing phase. The mechanism is re-engaged when the ankle reaches a maximum dorsiflexion angle just prior to the next step. The engagement and disengagement processes rely on the angular rotation of the ankle. Specifically, the mechanism requires a rotation of $7^{\circ}$ to switch states. 


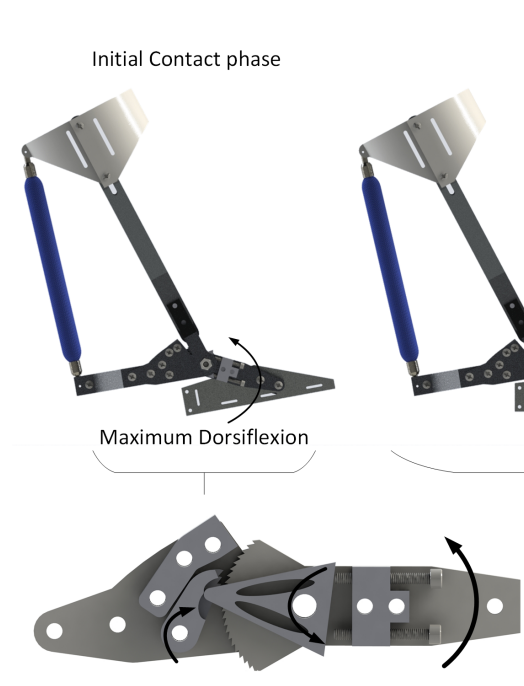

(A) Engagement
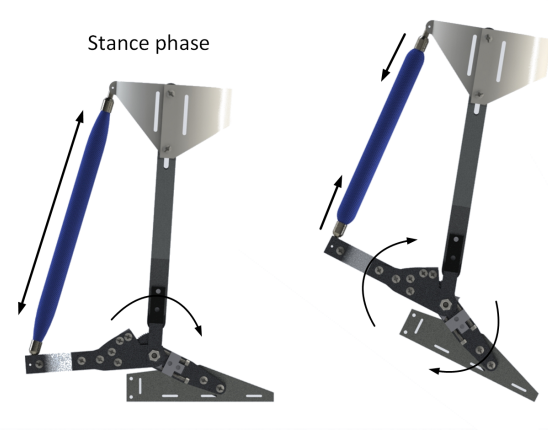

Push off phase

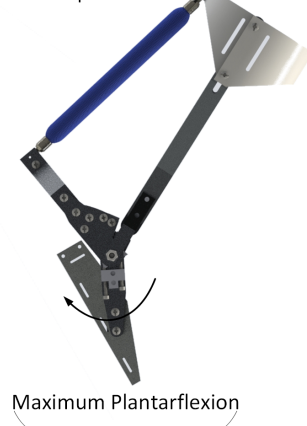

Maximum Plantarflexion

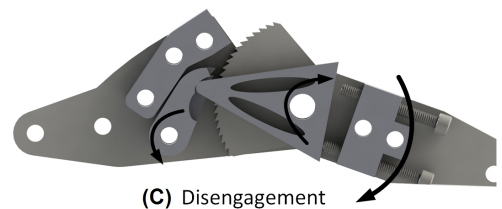

Figure 4.12: Illustration of the engagement and disengagement of the timing mechanism throughout the gait cycle.(A) shows the movement of the mechanism

from the disengaged to the engaged state. (B) shows the engaged state of the mechanism $(C)$ shows the movement of the mechanism from the engaged to the disengaged state

\subsection{Manufacturing}

In an attempt to keep the device as light as possible, 3D printed pieces made from ABS or PLA were used wherever possible. The initial prototype was made almost entirely by $3 \mathrm{D}$ printing. It was assembled using cylindrical pins that were lightly hammered into position and held by friction. Figure 4.13 presents the first complete prototype of the device. This initial version demonstrated the function of the timing mechanism. The main challenge encountered was the force transmission along the frame of the device when the spring element was attached. As the spring started to stretch, a problem became apparent. The spring could not be stretched more than a few millimeters before the frame and structural components began to deform under the load. Therefore, although the plastic frame and mechanism seemed adequately solid it could not transfer the force from the spring to the joint and vice versa. In order to reinforce the frame and improve force transfer, most of the plastic components were replaced by aluminum. 

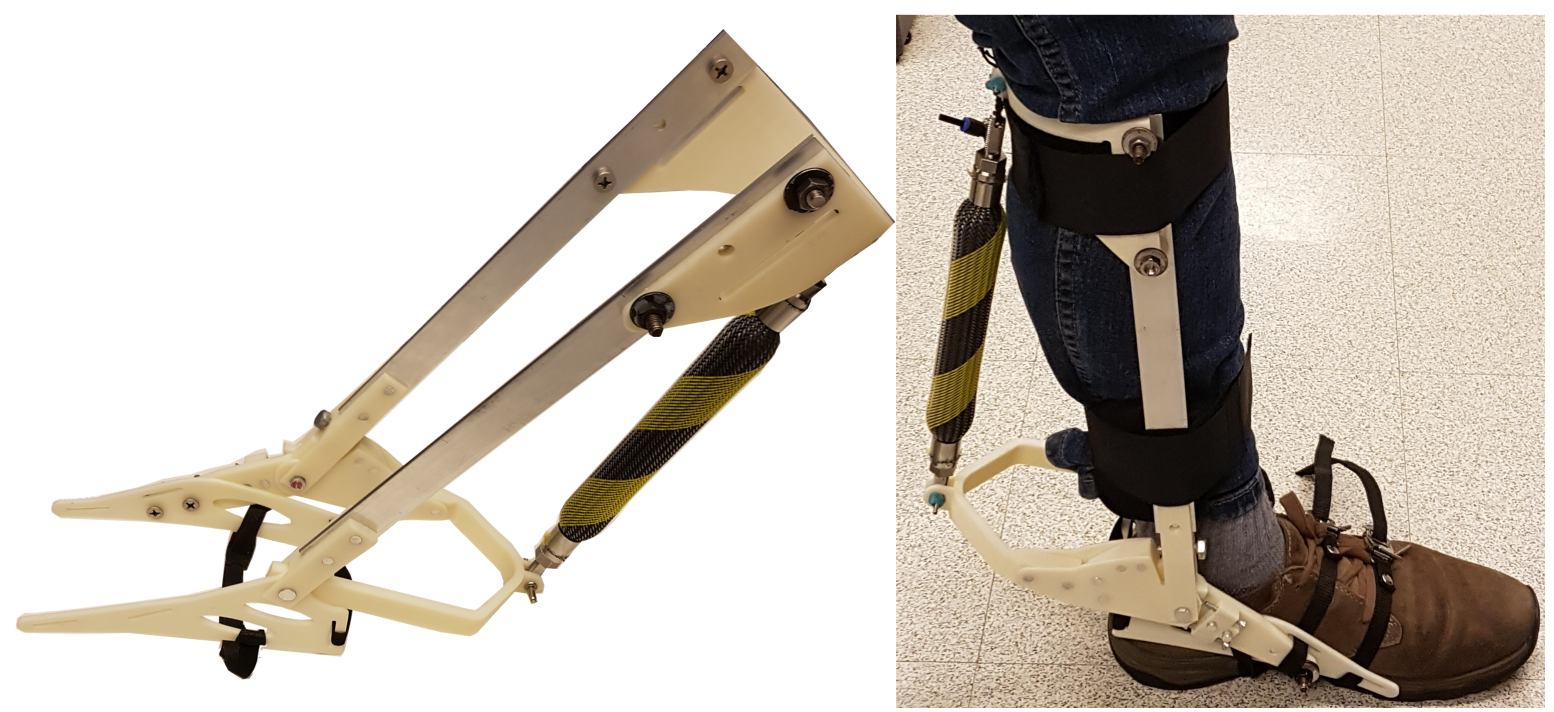

Figure 4.13: Photograph of the first complete prototype. White pieces were 3D printed from ABS. Upright pieces are made from aluminum

During the manufacturing process, certain design alterations needed to be made. Simple shapes were used to facilitate the machining. Furthermore, some of the larger of more complex pieces were made using 3D printing. The colour of the 3D printed pieces is related to the material that was currently available and has no other significance or impact on the device. The pieces in question are the timing components of the custom ratchet mechanism as well as the shank cuffs. These pieces experience relatively little stress and did not need to be made out of metal. However, due to the high forces in the ratchet gear and pawl, these were made out of aluminum and steel. The ratchet gear piece went through several 3D printed iterations before being abandoned. The final part was made out of aluminum and has a simplified geometry. Due to the long flat nature of the part, classical machining would have been difficult. Therefore, this piece was cut by hand and filed to shape. The ratchet pawl however was special ordered from ShapeWays 3D printing service. The part was 3D printed out of steel. The dimensional accuracy of 3D printing is not as good as machining. Therefore, the pawl, needed to be filed and sanded by hand slightly. Once completely assembled, preliminary testing was done. During the testing it was discovered that after a few minutes of use, the device shifted down and that the joint alignment was no longer acceptable. In order to keep the mechanical and biological joints aligned, the device needed to be kept from sliding down the leg towards the ground. This was accomplished by filling the space between the plates on either side of the foot and the floor using 3D printed stilts. The joint alignment is also ensured by 3 straps that wrap 
around the foot. One around the midfoot, one under the heel and one on the front of the ankle.

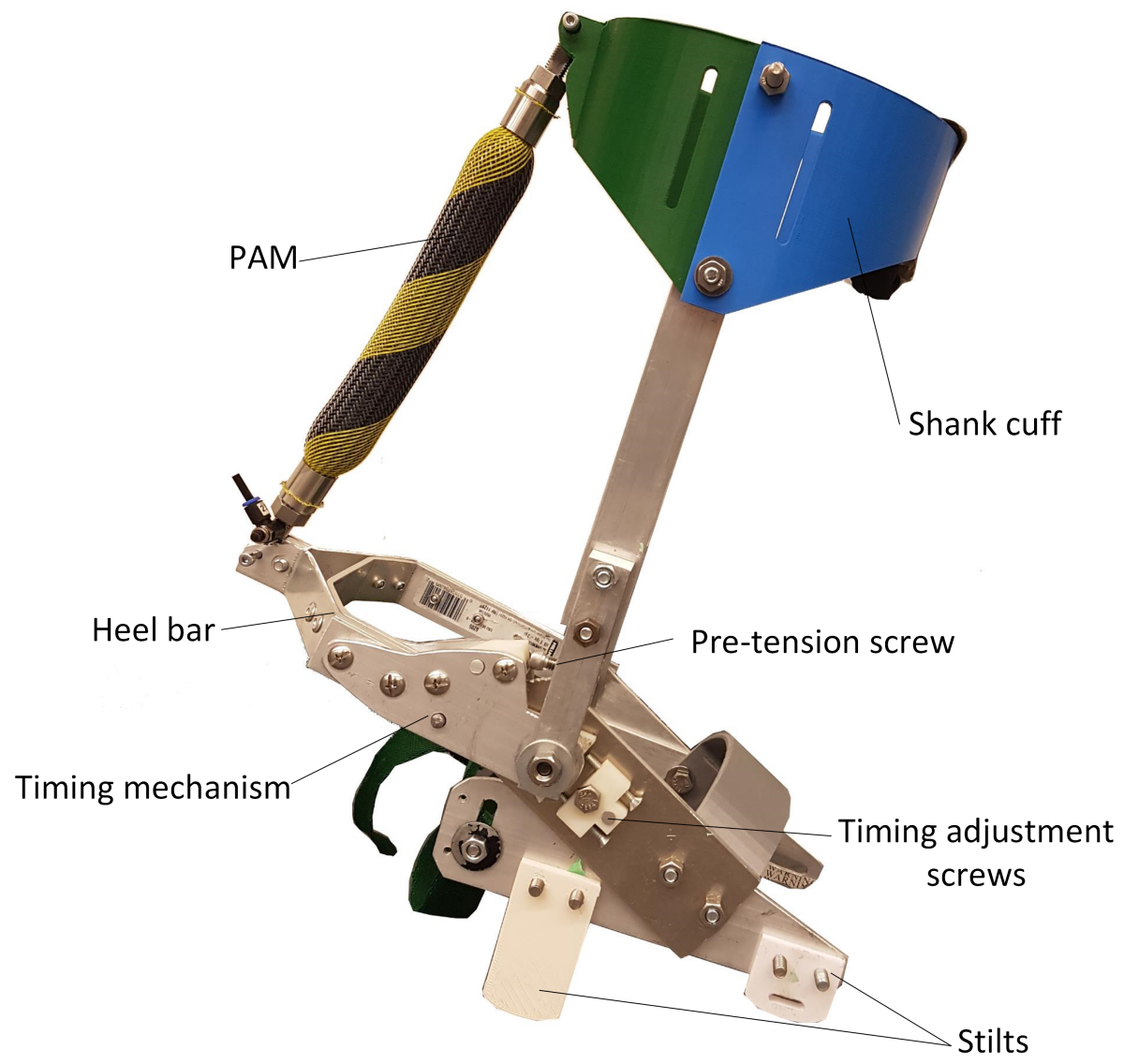

Figure 4.14: Photograph of the complete prototype.

The completed device was made primarily out of aluminum alloy with some 3D printed parts made out of PLA or ABS and a single component (the pawl) 3D printed out of steel. It weighs $1.51 \mathrm{~kg}$ which is well below the maximum of $4 \mathrm{~kg}$. Furthermore, it is able to reach well beyond the required $20^{\circ}$ of dorsiflexion and $50^{\circ}$ of plantarflexion. Thus, it should not interfere with the range of motion of the user. The simulations indicated that a PAM with 2 fiber revolutions is appropriate for the device. The timing mechanism ensures that the device does not interfere with the user during swing phase. The timing screws allow for precise adjustment of the ankle angles that trigger the mechanism. 


\subsection{Testing Procedures}

\subsubsection{Experimental Setup}

The mechanical testing of the prototype was carried out in order to analyze the ankle moment generated by the device. To achieve this, the exoskeleton was mounted in an Instron testing machine using a custom jig, see Figure 4.15.

The exoskeleton and jig were attached to each other and to the Instron machine using multiple bolts. The free end of the exoskeleton was placed directly underneath the $100 \mathrm{kN}$ static load cell. However, due to the rotation of the joint, the point of application does not travel in a purely vertical manner. To compensate for this, a flat attachment was used to apply a downward force while allowing horizontal displacement. The attachment is simply a flat steel surface against which the exoskeleton could slide, see Figure 4.15. As the cross head of the testing machine descended, the shank section of the exoskeleton rotated.

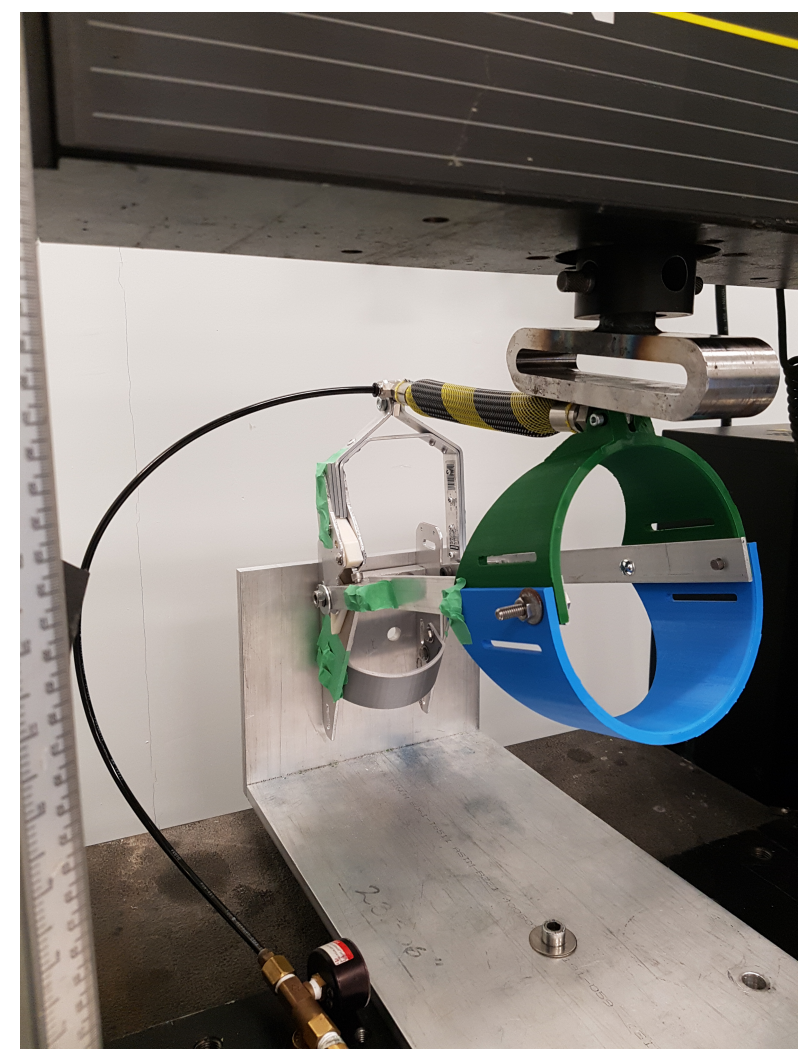

Figure 4.15: Photograph of the mechanical testing setup. The L bracket secures the foot plate while the shank section of the device is free to rotate. 


\subsubsection{Testing Parameters}

According to standard gait data, the loading of the Achilles tendon is done over approximately $15^{\circ}$ of dorsiflexion [6]. Using simple trigonometry, a displacement of $15^{\circ}$ would cause a linear translation of the free end of approximately $8 \mathrm{~cm}$. Therefore, the Instron machine was programmed to descend up to a maximum of $8 \mathrm{~cm}$.

Once the value of the $15^{\circ}$ rotation was known, the corresponding elongation of the spring was calculated. Given the desired ankle torque determined in Chapter 4, the initial pressure of the PAM was determined using the simulation presented in Chapter 3. The internal pressure required was estimated to be between $40-80 \mathrm{kPa}$. Furthermore, although the rotation of the biological ankle is $15^{\circ}$, it was thought that during use the ankle exoskeleton may not attain this rotation. Since the device is attached to the user with straps, there may be some relative movement that prevents the exoskeleton from perfectly following the rotation of the ankle. For this reason the device was tested at four different vertical displacements. The device was tested with vertical displacements of 5, 6,7 and $8 \mathrm{~cm}$. The testing parameters are shown in Table 4.2. For all tests the vertical displacement speed of the Instron machine was set to $2 \mathrm{~mm} / \mathrm{s}$. In an attempt to keep the torque produced by the exoskeleton within the desired range, the pressure inside the PAM was reduced as the vertical displacement of the test increased.

Table 4.2: Physical parameters and values related to the mechanical testing of the prototype exoskeleton. The muscle length refers to the distance between the attachment points, including the end caps

\begin{tabular}{lccc}
\hline Pressure & Muscle Length $\left(L_{m}\right)$ & Angle $\theta$ & Vertical Displacement \\
\hline \hline $70 \mathrm{kPa}$ & $0.286 \mathrm{~m}$ & $80^{\circ}$ & $50.3 \mathrm{~mm}$ \\
$70 \mathrm{kPa}$ & $0.286 \mathrm{~m}$ & $80^{\circ}$ & $60.4 \mathrm{~mm}$ \\
$55 \mathrm{kPa}$ & $0.290 \mathrm{~m}$ & $82^{\circ}$ & $70.3 \mathrm{~mm}$ \\
$48 \mathrm{kPa}$ & $0.295 \mathrm{~m}$ & $85^{\circ}$ & $80.3 \mathrm{~mm}$ \\
\hline
\end{tabular}

During the mechanical testing, the clutch mechanism was also examined. With the foot plate of the exoskeleton fastened to the testing plate, the changing angle of the device successfully engaged and disengaged the PAM. Furthermore, the adjustment screws functioned as intended and were used to increase and decrease the angles at which the PAM would be engaged and disengaged. 


\subsubsection{Data processing}

Once the mechanical testing was completed, the data acquired from the Instron linear testing machine needed to be converted into joint torque. This was achieved with a Matlab script, found in Appendix G. This script calculated the PAM elongation, force, as well as the moment produced around the ankle joint. Figure 4.16 illustrates the geometry of the device and the definition of angles $\theta$ and $\beta$.

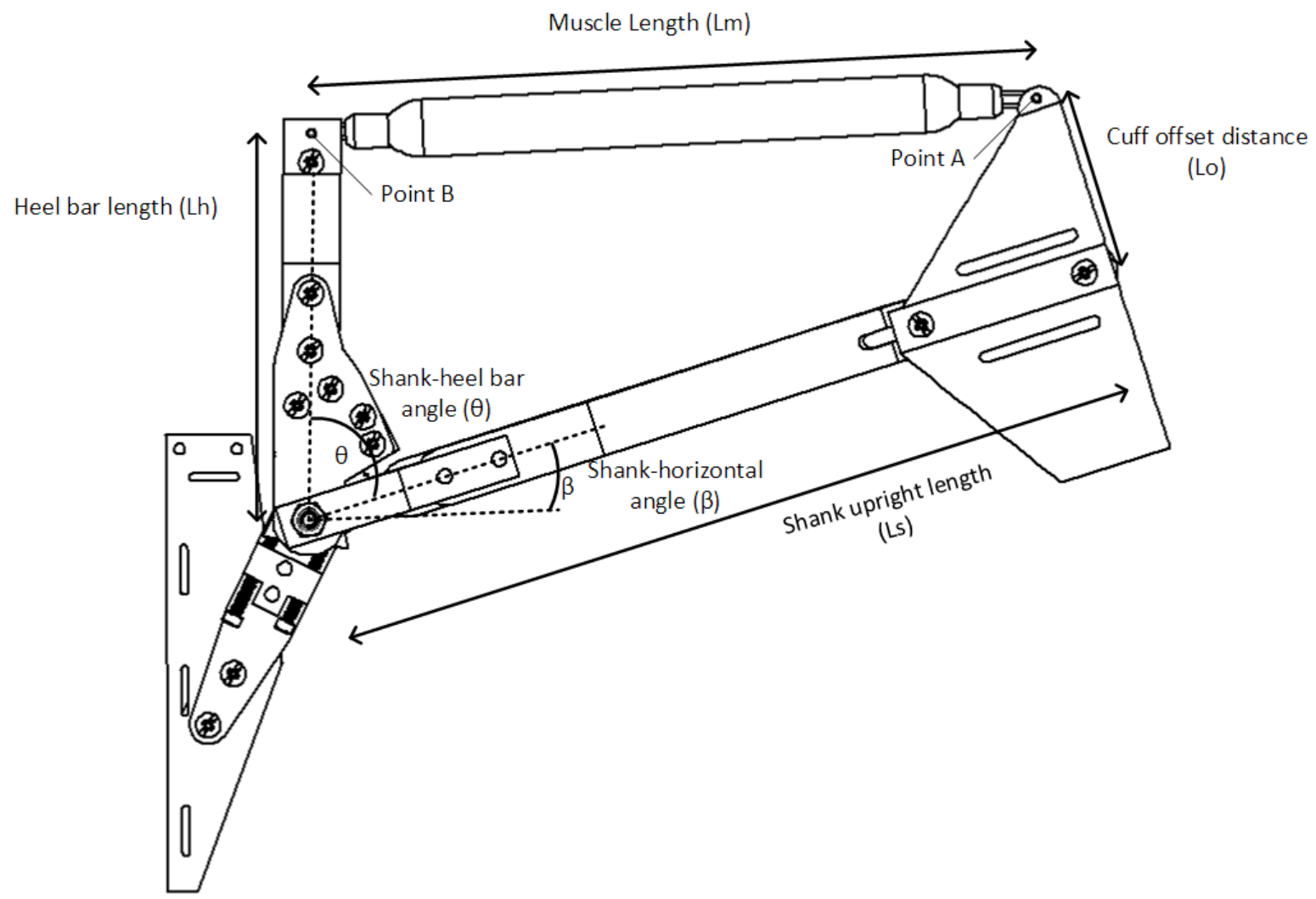

Figure 4.16: Illustration of the side view of the device with member lengths and angles identified.

The script first set the ankle joint as the origin. The position of point A was then calculated. Using the free body diagram shown in Figure 4.17, and the known force applied by the Instron machine $\left(F_{\text {Instron }}\right)$, the force in the pneumatic muscle $\left(F_{P A M}\right)$ was calculated. A quasi-static assumption was adopted for this analysis given the low speed used for the Instron machine. 


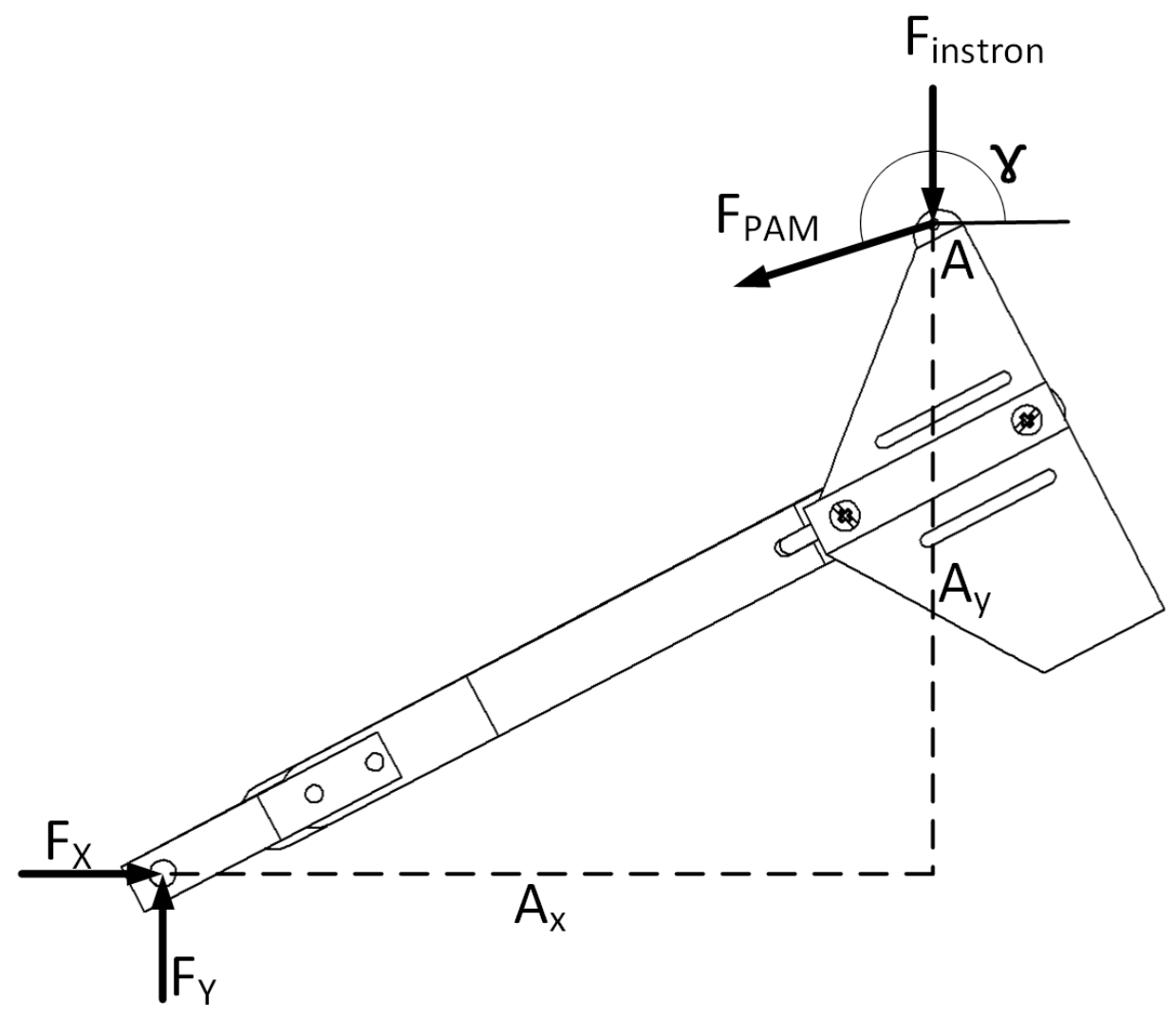

Figure 4.17: Free body diagram of the shank section of the ankle exoskeleton device. Point $\mathrm{A}$ is the attachment point of the PAM and the shank cuff. Values $A_{x}$ and $A_{y}$ are the horizontal and vertical distances between the ankle joint and point A. Angle $\gamma$ defines the position of the PAM with respect to horizontal

Once the force in the PAM was known, the other attachment point was examined. This was referred to as point B. Figure 4.18 shows the free body diagram of the heel bar. Using the calculated force in the PAM, the moment acting at the ankle joint was calculated. It was this moment that was used to estimate the contribution to the human ankle moment. 


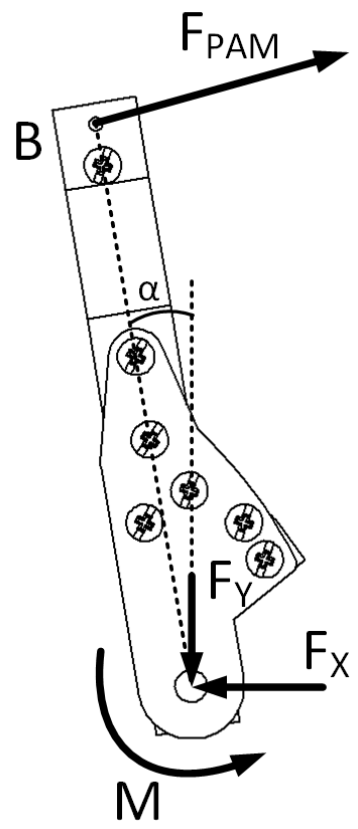

Figure 4.18: Free body diagram of the heel bar section of the ankle exoskeleton device. Point $B$ is the attachment point of the pneumatic muscle. Angle $\alpha$ is the angle between the heel bar and vertical. During testing this angle was kept constant at $5^{\circ}$

\subsection{Results}

The mechanical testing results are presented in this section. The results demonstrate the ability of the exoskeleton to exceed the mandated $25 \%$ of the biological contribution with exception of one test.

Figures 4.19 and 4.20 present the force vs elongation and the joint torque graphs for the exoskeleton as well as the hypothetical ideal spring and the natural ankle torque. 


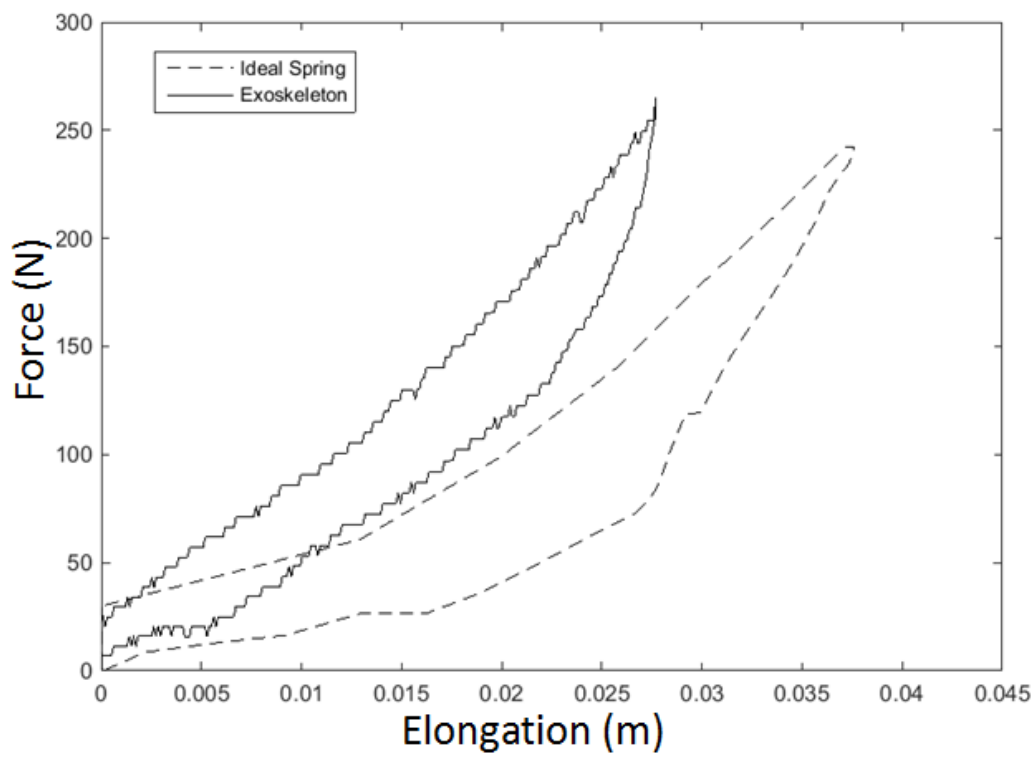

Figure 4.19: Force vs elongation graph of the mechanical testing of the PAM compared with the theoretically ideal spring. The PAM is inflated to $70 \mathrm{kPa}$ and the vertical displacement of the Instron cross head is $5 \mathrm{~cm}$.

With reference to Figure 4.19, the maximum magnitude achieved by the exoskeleton and the predicted ideal spring is similar, however the slope of the curves is different. This slope corresponds to the stiffness of the spring element. The steeper slope indicates a stiffer spring. In this case, the PAM is stiffer than the ideal spring. Figure 4.20 demonstrates that the moment profile generated by the biological joint and the exoskeleton are similar both in terms of magnitude and shape. Note that the experimental data was adjusted so that the horizontal axes of the two cases match. The elongation rate was kept constant on the Instron machine, and therefore, the time required to complete a cycle increased as the vertical displacement of the Instron cross head increased. 


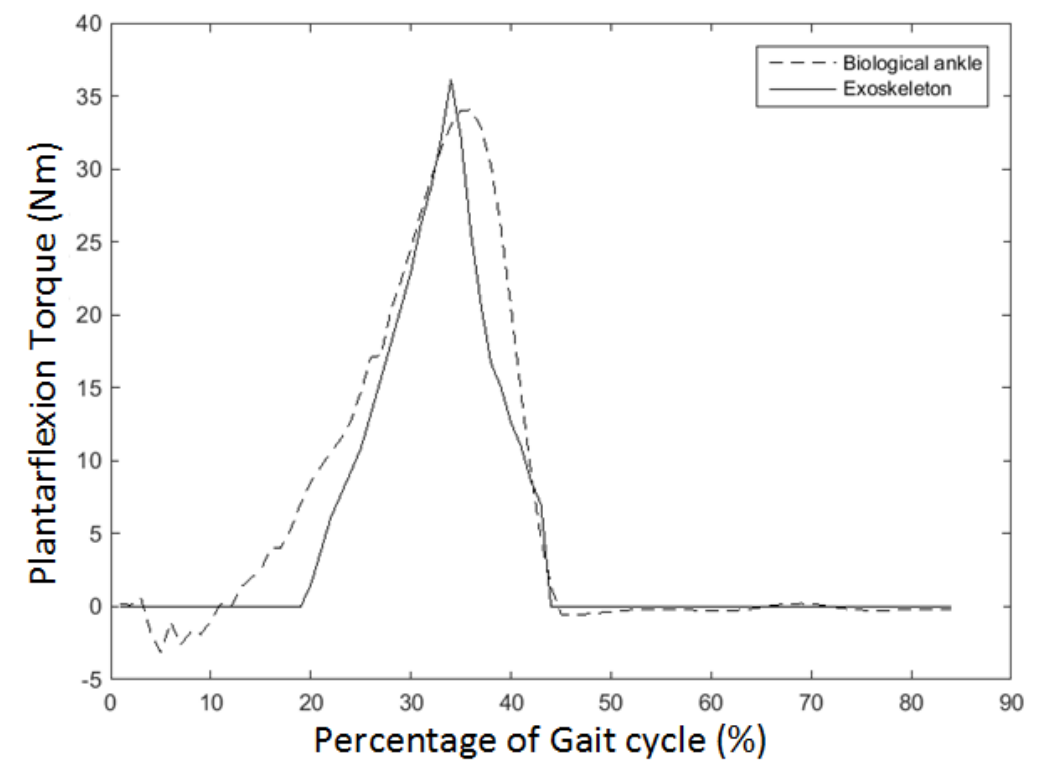

Figure 4.20: Joint torque vs percentage of gait cycle of the mechanical testing of the PAM compared with the theoretically ideal spring. The PAM was inflated to $70 \mathrm{kPa}$ and the vertical displacement of the Instron cross head was $5 \mathrm{~cm}$.

According to the testing parameters, the maximum vertical displacement of the Instron cross head was $8 \mathrm{~cm}$. Figure 4.21 presents the force vs elongation curve of a PAM inflated to $48 \mathrm{kPa}$ when the cross head was set to move $8 \mathrm{~cm}$ vertically. 


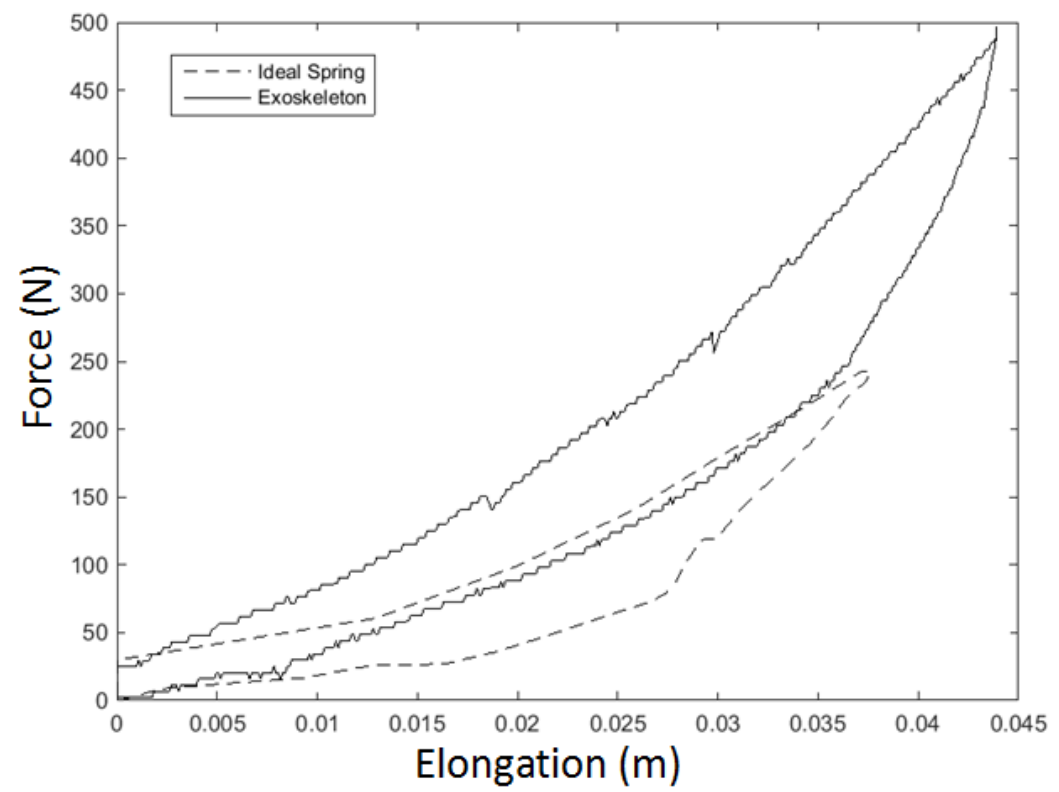

Figure 4.21: Force vs elongation graph of the mechanical testing of the PAM compared with the theoretically ideal spring. The PAM was inflated to $48 \mathrm{kPa}$ and the vertical displacement of the Instron cross head was $8 \mathrm{~cm}$.

Figure 4.21 demonstrates that the force generated by the PAM significantly surpasses the required $25 \%$ of the ideal spring.

In the case of the joint torque, the results are similar. Figure 4.22 shows the joint torque of a PAM inflated to $48 \mathrm{kPa}$ and subjected to a vertical displacement of the cross head of $8 \mathrm{~cm}$. Once again, the experimental value significantly surpasses the required $25 \%$. 


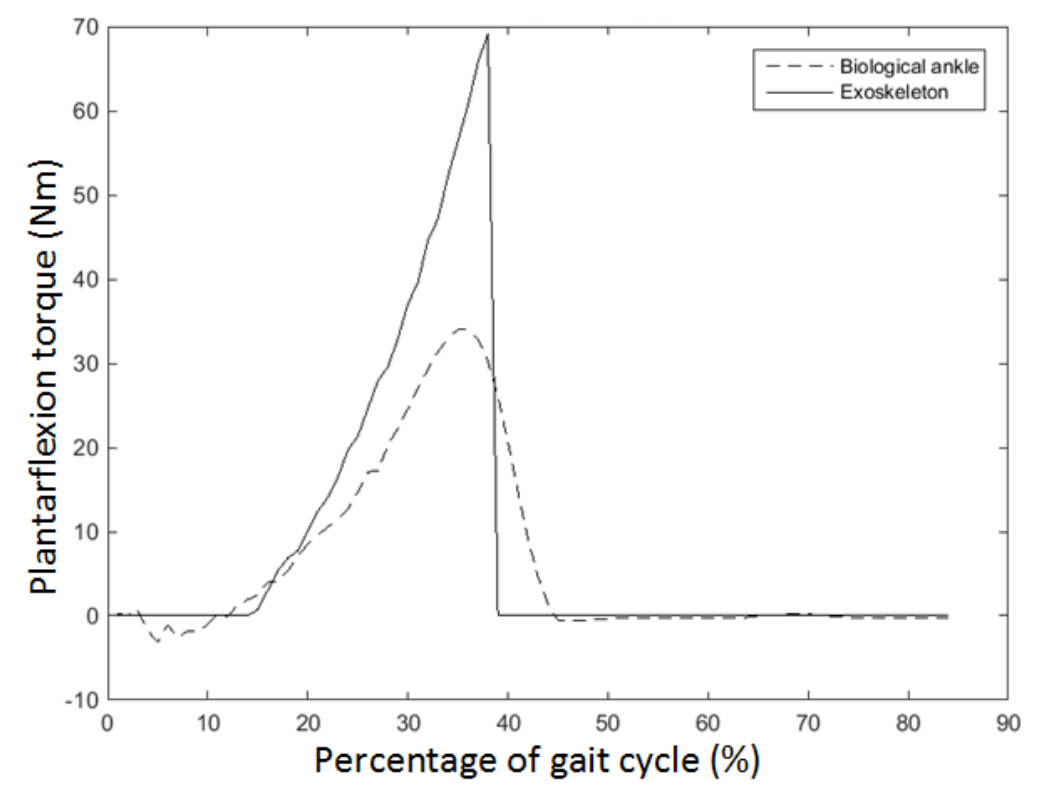

Figure 4.22: Joint torque vs percentage of gait cycle of the mechanical testing of the PAM compared with the theoretically ideal spring. The PAM was inflated to $48 \mathrm{kPa}$ and the vertical displacement of the Instron cross head was $8 \mathrm{~cm}$.

For comparison purposes, the contribution of the ideal spring was increased until it matched the experimental values. If the percentage was changed to $50 \%$ of the normal biological ankle torque, the fit was much better, see Figure 4.23 




Figure 4.23: Joint torque vs percentage of gait cycle of the mechanical testing of the PAM compared with the theoretically ideal spring. The PAM is inflated to $48 \mathrm{kPa}$ and the vertical displacement of the Instron cross head is $8 \mathrm{~cm}$. The ideal spring force is set to $50 \%$ of the natural biological torque

From the results presented in this section, the PAM inflated to $70 \mathrm{kPa}$ and stretched with a vertical displacement of the Instron cross head of $5 \mathrm{~cm}$, was best for replicating $25 \%$ of the biological ankle torque. However, this displacement does not capture the entire range of motion of the ankle joint during walking. The calculated $8 \mathrm{~cm}$ of vertical displacement was found based on the assumption that the exoskeleton moved through the entire range of motion of the user. Furthermore, it also assumed that the gait pattern did not change and that the user reached this full range of motion. It is impossible to predict how much play will be present in the attachment of the device to the user during human testing. Therefore, the actual range of motion of the device could very well be closer to $5 \mathrm{~cm}$ than it is to the theoretically perfect value of $8 \mathrm{~cm}$. For this reason, the human testing used a PAM inflated to 70 and $55 \mathrm{kPa}$. 


\section{Chapter 5: Human testing}

The proposed ankle exoskeleton is intended to assist human walking, therefore, the ultimate test of functionality is human walking trials. These trials examined the mechanical function of the device as well as the muscular effort of the user. The tests used motion capture to analyze the movements of the user and electromyography to quantify the muscle activation. Furthermore, this chapter explains the testing procedures as well as the results and the limitations of the achieved tests. 


\subsection{Ethics Approval and Recruitment}

The University of Ottawa Office of Research Ethics and integrity has approved the human testing described in this section. The certificate of approval, file number H09-16-05, can be found in Appendix F.

\subsubsection{Participants}

For this work 5 participants were tested, 3 male and 2 female. The participants had an average age of 22.2 years, an average height of $1.73 \mathrm{~m}$ and an average weight of $70.4 \mathrm{~kg}$, with standard deviations of 2.4 years, $0.14 \mathrm{~m}$, and $15.7 \mathrm{~kg}$. The participants were selected from the University of Ottawa student population, and were required to be at least 18 years of age and be in good general health. They were required to have no history of lower limb injury that would affect their gait. It was also required that the participants not have any current lower limb injuries. To be considered no longer current, injuries were required to be symptom free for at least one month. Since only one prototype was manufactured some participants were excluded because the device simply did not fit them.

\subsection{Testing Protocols}

\subsubsection{Motion Capture}

Kinematic data were recorded using 16 Optitrack V100:R2 cameras controlled by Motive software. Both the cameras and the software are made by NaturalPoint Inc. A full system calibration was performed once daily before the testing sessions began. If multiple participants were tested in the same day, the system was not re-calibrated between participants. For all calibrations, the cameras recorded at least 1000 frames of usable data and the mean error was of less than $0.3 \mathrm{~mm}$.

\section{Marker Set : Helen Hayes}

Reflective markers were placed on the participant following the lower body Helen Hayes $(\mathrm{HH})$ marker set built into Motive. This set uses a total of 19 markers. The 
pelvis is described using three markers. One on the anterior superior Iliac Spine both left and right, and one marker on the sacrum. Moving down to the thigh, one marker is placed on the outside of each thigh approximately $2 / 3$ of the distance from the Greater Trochanter to the Femoral Epicondyle. There is then one marker on the Lateral Epicondyle of the Femur, as well as one on the Medial Epicondyle of the Femur. Next, there is a marker on the outside of the shank approximately half of the distance between the knee and ankle. The ankle joint is represented by a marker on the Lateral and Medial Malleolus. For the foot, one marker is placed on the posterior surface of the Calcaneus, and one marker on the head of the second Metatarsus. There are 8 markers per leg and 3 on the pelvis for a total of 19, see Figure 5.1. The markers were $15.9 \mathrm{~mm}$ in diameter and held in place using a double sided adhesive tape.

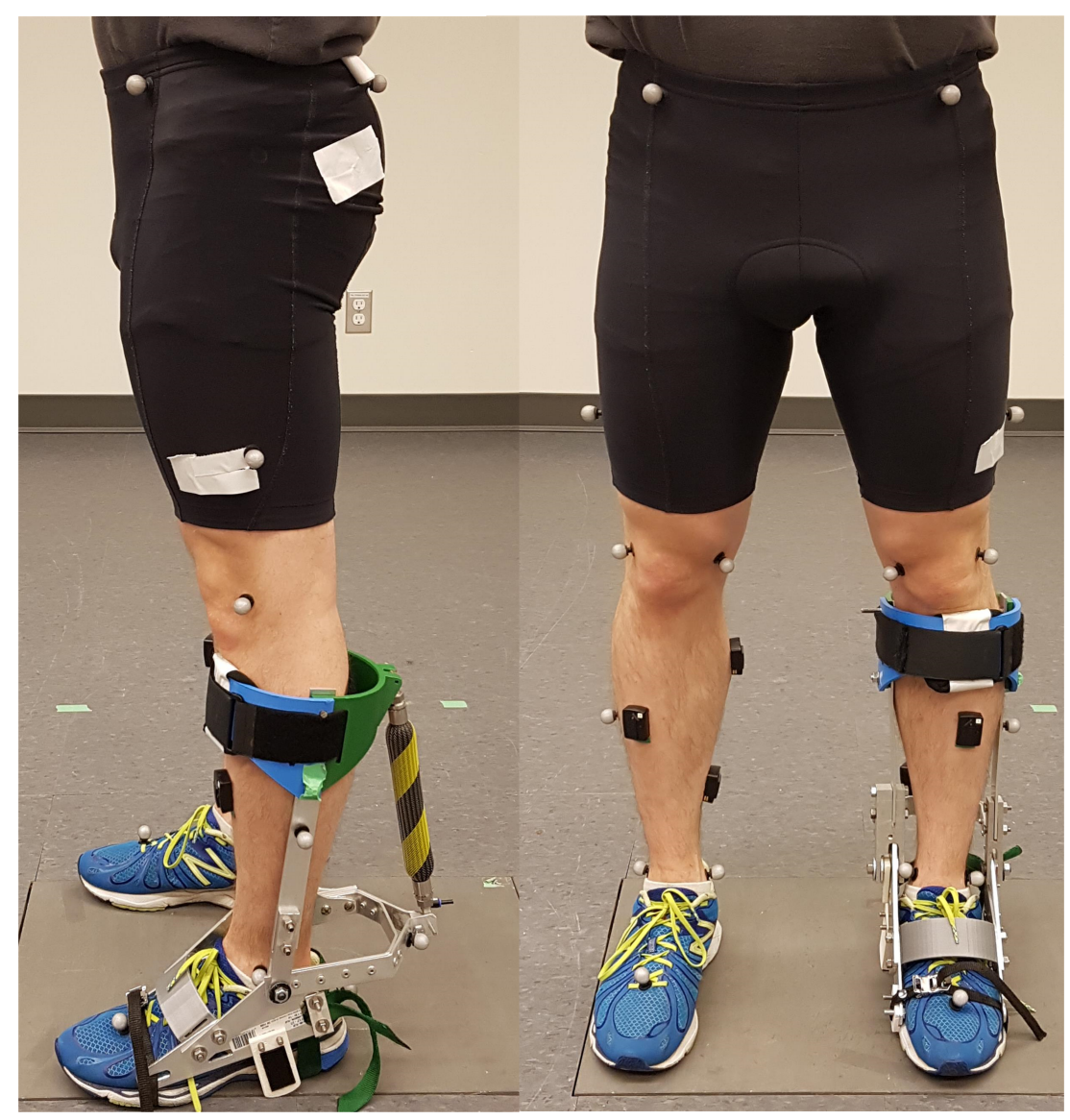

Figure 5.1: Photograph of the legs of a participant once the exoskeleton, the EMG sensors, and the reflective markers were placed

When the exoskeleton was worn, the device interfered with some of the markers and prevented the cameras from seeing them clearly. Therefore, the marker set was modified slightly. Once the $\mathrm{HH}$ marker set was recognized by the Motive software, 
the two Maleolus markers on the leg with the exoskeleton were moved to the outside of the device. These were placed as close to the joint axis as possible. These markers were still be used to re-construct the ankle joint and were only moved because the camera system had trouble detecting the markers since they were partially obscured by the exoskeleton. The new marker positions were added to the HH model and saved as a new custom skeleton in the Motive software. Two additional markers were placed at the top and bottom of the spring element, this allowed the instantaneous length of the PAM to be recorded.

\subsubsection{Electromyography (EMG)}

A Delsys Trigno ${ }^{\mathrm{TM}}$ wireless EMG system was used to collect data related to the muscle activation. These wireless sensors have integrated accelerometers which were important for the synchronization of the EMG and motion capture data. A total of 6 surface electrodes were placed on the legs. Three electrodes per leg. The electrodes were placed on areas of clean, dry, hairless skin following the SENIAM protocol [56] as closely as possible. This protocol describes various muscles and suggests the location of EMG sensors. The exoskeleton device covered a significant portion of the leg, as such, some EMG sensors had to be placed slightly differently to avoid being disrupted during walking. The muscles examined were the Tibialis Anterior, the Gastrocnemius Medialis and the Soleus. The electrodes were wirelessly connected to the control box that was in turn connected to a PC. The PC was running the Delsys EMG Works acquisition software. The EMG sampling rate was $2000 \mathrm{~Hz}$.

In order to compare values between participants the EMG data was normalized using the Maximum Voluntary Isometric Contraction (MVIC) values which were obtained using two tests. One test for the Tibialis Anterior, and the second test was used to calculate the MVIC for both the Gastrocnemius Medialis and the Soleus. For the Tibialis Anterior MVIC trial, the participant was asked to stand comfortably and try to lift the ball of one foot off the ground. During this, the lead researcher was firmly holding the participant's toes against the floor and preventing any motion of the foot by placing one hand on the top of the foot and applying pressure. This test activated the Tibialis Anterior muscle as the participant attempted to dorsiflex the foot. The test for the Gastrocnemius and Soleus muscles was a static calf raise on one foot. The participant was asked to stand on one foot with both hands on a railing in front of them. They were then instructed to keep their heel about 1 inch off of the ground and 
try to lift the railing (which was bolted to the wall) by only using their calf muscle. Each MVIC test lasted 5 seconds and was performed twice.

\subsubsection{Experimental Protocol}

When participants arrived they were presented with the consent form, and were asked to read it carefully. During this time, the researchers were available to address any questions the participant might have. The participant was then asked to change into tight fitting shorts and sport shoes. Since no markers were placed on the upper body, the type of shirt worn by the participant was not important so long as it did not cover the markers placed on the pelvis. The participant was then fitted with the device. The researchers asked the participant to sit on a chair while the device was adjusted and tightened. The attachment of the device is composed of a series of straps; these were tightened to be snug but not painful. The participant was then given 10 minutes to warm up and walk around to familiarize themselves with the device. During this time, the researchers observed and adjusted the device if needed. The participant was then asked to sit down again so that the EMG sensors could be placed. Figure 5.2 shows the legs of one participant wearing the device and the EMG sensors. The EMG sensors are surface electrodes, thus to ensure a good reading the skin surface needed to be prepared. The researchers selected the areas where the sensors would be placed. The areas were then shaved and cleaned with an alcohol wipe. The EMG sensors were attached to the clean, dry, hairless skin using a double sided adhesive tape. 


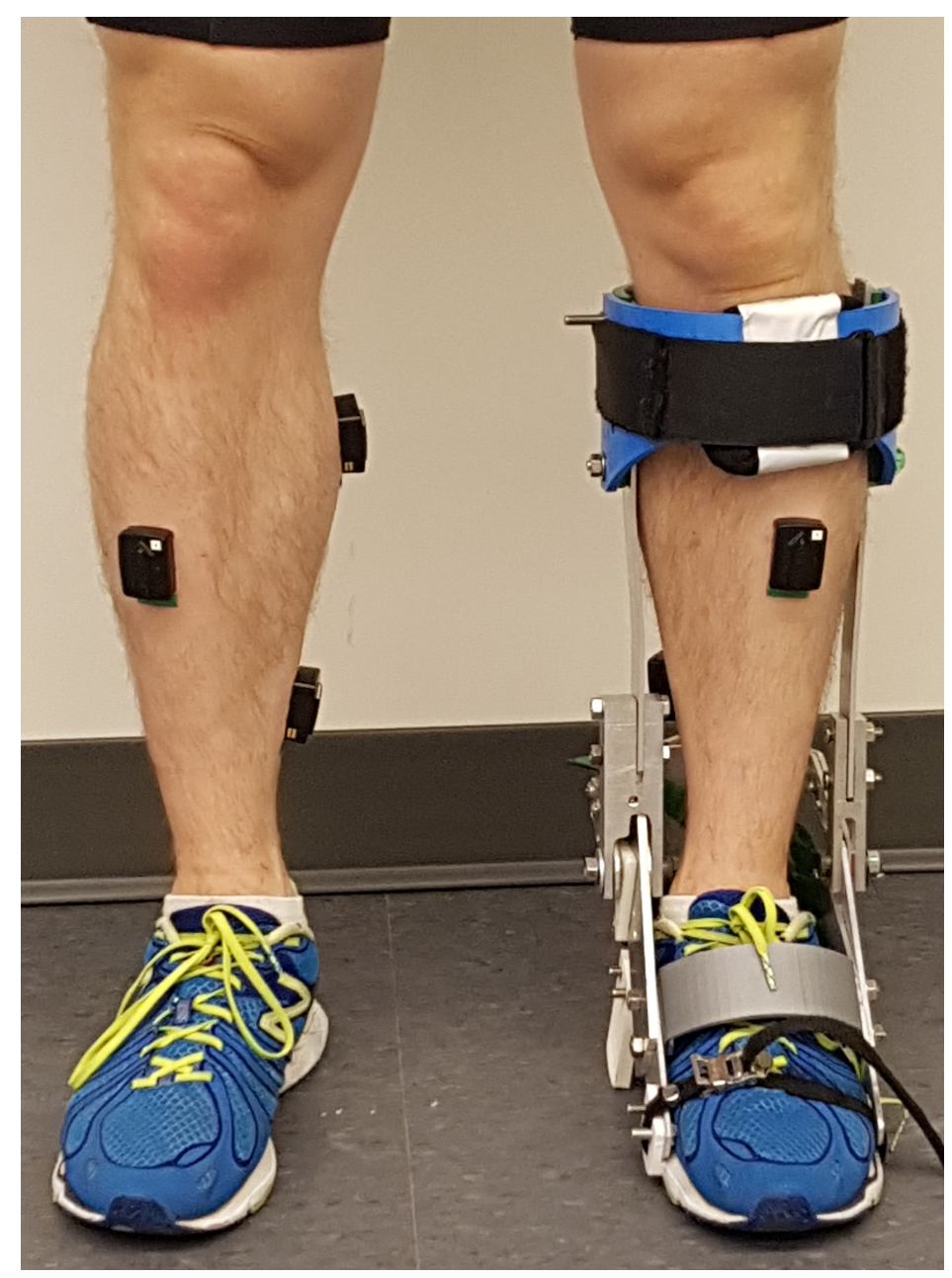

Figure 5.2: Photograph of the placement of the EMG sensors on the legs of a participant wearing the exoskeleton

The testing began with MVIC trials for each targeted muscle to establish baseline values for participant specific maximum signal amplitude. During these tests, the exoskeleton device was worn but no spring element was connected so that the range of motion of the user was not affected. After the MVIC trials, the researchers placed the reflective markers required for the motion capture. Next, the participant was asked to stand in the capture volume so that the Motive software could construct a skeleton model of the participant. As described previously, this involved beginning with a $\mathrm{HH}$ maker set then modifying it to generate a custom skeleton model. The last step required before the walking trials was a static calibration recording. For this, the participant was asked to stand still while several seconds of motion capture data were recorded. This was required for the kinematic analysis.

Once all preliminary and calibration tests were completed, walking trials were 
conducted. The participant was told to walk at a normal comfortable pace. No instructions or recommendations that would alter the gait of the participant were given. The participant was asked to practice walking several times. The researchers adjusted the starting position within the lab to ensure optimal footfall positioning during the test. Since the participant is starting from a still stand, the first few steps are not representative of the regular gait pattern. Therefore, the researchers ensured that the $4^{\text {th }}$ and $5^{\text {th }}$ steps were still within the motion capture area so that they could be used during data processing. Since the motion capture and EMG systems operate independently, they needed to be synchronized in post processing. To achieve this, the participant was asked to perform a small hop every trial before beginning to walk. The synchronization method will be explained further in the next section.

A total of five walking tests were done, each with a different exoskeleton configuration or PAM pressure. Each test included 7 to 10 trials. The order of tests was as follows: $\mathrm{PAM}$ at $70 \mathrm{kPa}$, and $40 \mathrm{kPa}$, a soft elastic band, no spring element and finally for the last test, the exoskeleton was removed and the participant was asked to walk normally. The tests were always done in this order. Initially the baseline walking trials (without exoskeleton) were done first. However, it was quickly discovered that putting on the exoskeleton after having placed the EMG sensors and motion capture markers was quite a challenge. For the tests to be comparable to one another, it is important that the sensors and markers not be moved between tests. By conducting the exoskeleton testing first, the device could then be partially disassembled to be removed, thus not disturbing the sensors or markers.

The tests chosen were justified as follows. The PAM was tested at two different inflation pressures to examine the effect of varying stiffness. The Elastic band was very compliant and provided little assistance. It was used solely to keep the heel bar from swinging uncontrollably during walking. This test was intended to demonstrate the effect of the weight of the device while it is providing no assistance. The test in which the spring element was completely removed represented the worst case scenario. Not only did the user have to compensate for the additional weight of the device, but also for the unpredictable motion of the heel bar. Without a spring element to pull the heel bar up against the stopper block, the movement of the ankle moved the heel bar with it. In this configuration, the timing mechanism cannot function properly since there is no force that moves the heel bar into the engagement position. This was based on preliminary testing during which the participant stated that the balance of the foot felt strange when no spring element was connected, thus 
it was deemed valuable to test.

During the human testing, it was noticed that the timing device did not function as intended. More specifically, the spring was engaged throughout the entire gait cycle. However, despite this, the device did not hinder the user during the swing phase. The imperfect fit of the device, and the fact that there was relative movement between the device and the user, led to a certain amount of play before the user began to fight the device. This play was sufficient to allow the user to move their limb through the swing phase normally.

\subsubsection{Data Analysis}

\section{Kinematics Analysis}

The motion capture data collected during the testing was used to examine the kinematics of the gait. This was achieved using Visual 3D (C-Motion).

The kinematic analysis began by exporting the motion data from Motive. The exported data started with the heel contact of the right foot and ended with the second heel contact of the left foot. Therefore, every data file included a complete gait cycle for the right and left foot.

In the Visual 3D software, a skeleton model was created using the static calibration data that was recorded prior to the walking trials. This consisted of associating markers with anatomical landmarks to create a model of the participant. The testing also included placing markers on the top and bottom of the spring element so that its length could be calculated. In order to do this, a hypothetical muscle was created between the two points. The length of the muscle was then calculated. The motion capture data was filtered using a bidirectional butterworth filter and averaged across 7 trials and was plotted. The calculated variables were the angles of the ankle knee and hip joints as well as the length of the spring element.

\section{EMG Analysis}

Following the data collection, the recorded muscle activation signals were analyzed using a custom MatLab script. The data was first passed through a Butterworth high pass filter with a cut off frequency of $15 \mathrm{~Hz}$. The signal was rectified before pass- 
ing through a Butterworth lowpass filter with a cut off of $8 \mathrm{~Hz}$. To normalize the data, the MVIC signals were used. The MVIC trial was divided into $50 \mathrm{~ms}$ segments and averaged. The maximum amplitude of each muscle was selected as being the largest of the mean values.

Following the MVIC normalization, the walking trial data was trimmed to a single gait cycle then averaged over multiple takes. The full gait cycle was measured from heel strike to ipsilateral heel strike. To identify the heel strike events, the Motive motion capture software was used. The heel strike events were manually identified based on the marker tracking. The individual sample frames corresponding to the heel strike events were recorded for both the right and left legs. However, the EMG data was recorded in a separate software. Therefore, the frames corresponding to the gait events were only applicable to the data obtained by the motion capture system. In order to identify what muscle voltage corresponded to what phase of gait, the EMG data needed to be synchronized with the motion capture data. To do this, the hop movement at the beginning of the trial was used. In the motive software there is a function that allows the user to track the 3D position of any marker individually. When the participant performed a calf raise or small hop at the very beginning of the walking trial, the acceleration of the EMG sensor was paired with that of the markers.

In motive, the marker placed on the lateral Femoral Epicondyle was used. The position of the marker was examined in the vertical direction during the calf raise. The sample frame in which the marker reaches the apex of the hop was manually recorded in a notebook. Next, the vertical acceleration of the EMG sensor on the Tibialis Anterior was examined, using the integrated accelerometer. At the apex of the hop, the vertical acceleration of the marker was 0 since it had finished rising but had not yet begun to fall. Therefore, the time (in seconds) when the vertical acceleration passed through the $\mathrm{X}$ axis was recorded in a notebook.

At this point in the processing, the MVIC values for each muscle had been extracted, the motion capture frames corresponding to the hop, as well as the heel strike events for a full gait cycle of the left and right leg were known. With this information, the custom Matlab script paired and trimmed the motion capture and EMG files so that they both started time 0 at the apex of the synchronization hop. The time elapsed between the synchronization event and each heel strike event was calculated. The time between the synchronization event and the heel strike events was converted into sample frames in the EMG file. The EMG file was then trimmed to isolate one full gait cycle for the right and left legs respectively. Next, the trial was converted from 
time to percentage of gait cycle. However, the duration of a single gait cycle varied, thus, the number of samples found in a single gait cycle is different between takes. The data was fitted with a spline curve that passed through all of the data points. This was then used to generate data based on a constant base of 100 samples. The trials were then averaged at each point to represent the average gait pattern of the individual for that test.

The average muscle activation was examined over the entire gait cycle. The muscle activation was integrated for each muscle to provide a numerical value of the averaged total muscle contraction. Using the baseline walking test, these values were then represented as percentages of variation of the baseline values.

\subsection{Results}

The kinematic data obtained from the motion capture as well as the EMG results are presented in the form of graphs and tables. The tables included in this work include all five participants, however the complete set of motion capture and EMG graphs is presented for participant 1 only, and can be found in Appendix E.

\subsubsection{Motion Capture Results}

The purpose of the motion capture analysis was to generate kinematic data of the lower limbs in order to determine if the gait pattern of the individual was significantly different between tests.

In order to examine the effect of the exoskeleton, the normal gait pattern of the individual must be known. Figure 5.3 presents the angle of the ankle during a single gait cycle for the baseline (normal) walking trial of participant 1 . The plotted line is the mean value of seven trials and the shaded area surrounding it is the standard deviation. 

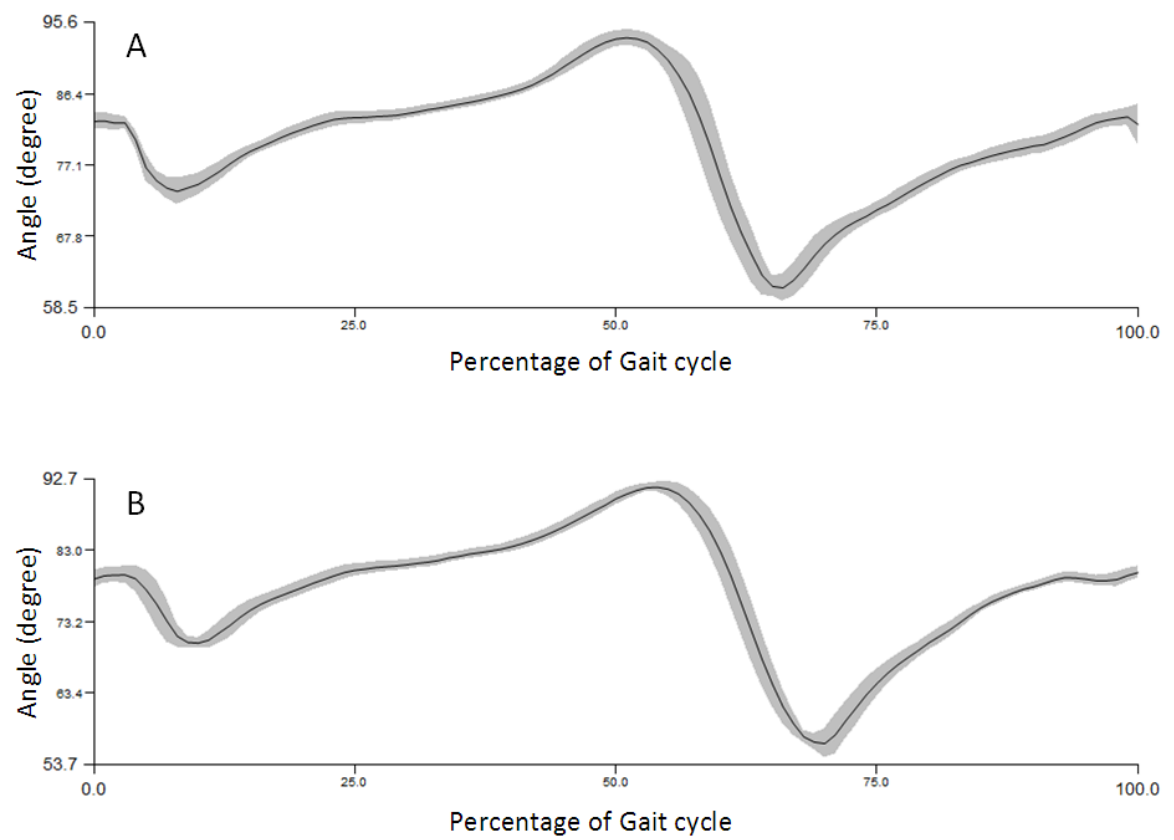

Figure 5.3: Ankle angle for baseline walking trial (without exoskeleton) A) Right leg, B) Left leg

Figure 5.3 shows that the angle of the ankle has a maximum angular variation $32.4^{\circ}$ for the right ankle and $34.8^{\circ}$ for the left ankle. Furthermore, the angular rotation of the ankle joint throughout the gait cycle are practically identical for the right and left ankles.

The results of the trial with the PAM inflated to $70 \mathrm{kPa}$ for are presented in Figure 5.4. The exoskeleton was worn on the participant's right foot. 

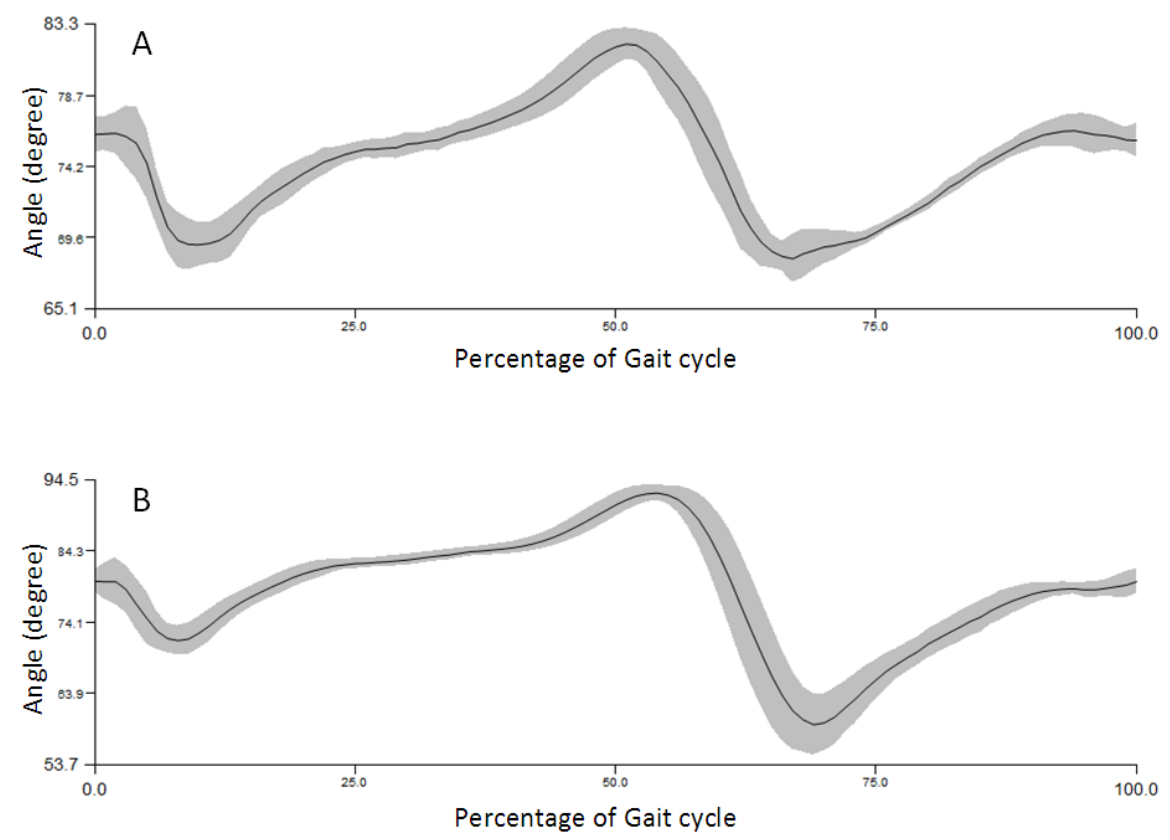

Figure 5.4: Walking trial ankle angle for exoskeleton with PAM inflated to $70 \mathrm{kPa}$ for participant 1. A) Right leg (wearing exoskeleton), B) Left leg (without exoskeleton)

From Figure 5.4 graph A), the maximum angular variation of the right ankle is reduced to $15^{\circ}$. Furthermore the angle variation of the left ankle is 33.7 which is very similar to the baseline values. Therefore, the exoskeleton reduced the range of motion of the right ankle however, the left ankle was minimally affected, this was true for all participants.

The analysis of the data also included tracking the length of the PAM throughout gait. Figure 5.5 presents the length of the PAM for the trial in which the PAM was inflated to $70 \mathrm{kPa}$. 


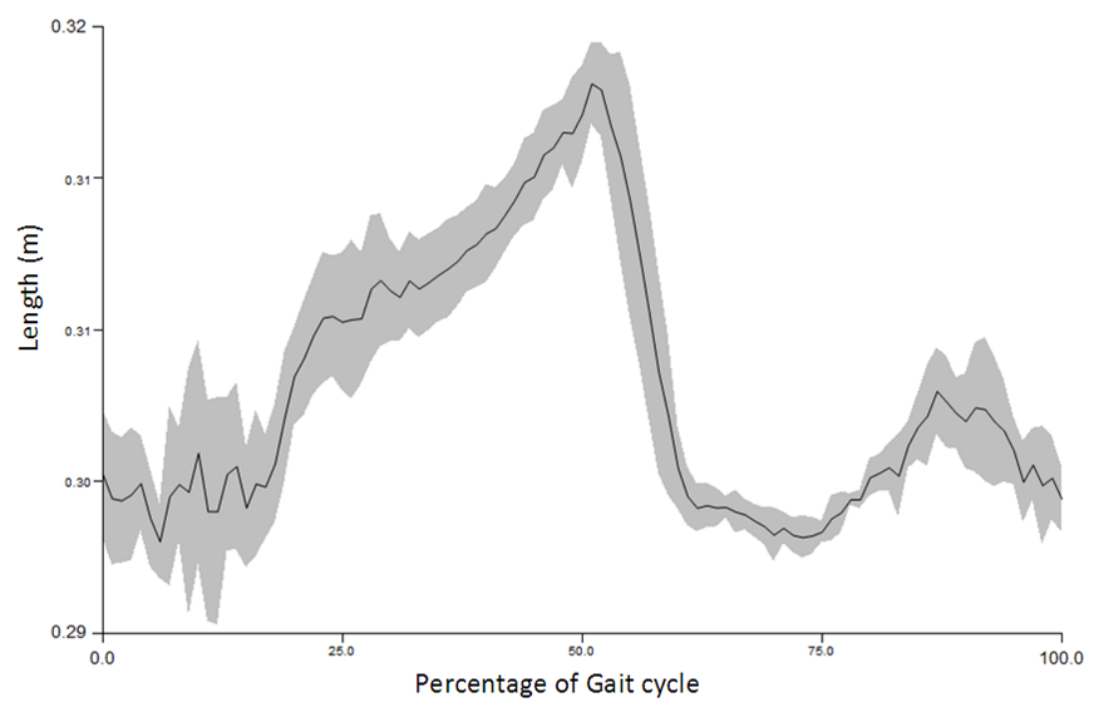

Figure 5.5: Walking trial spring length for exoskeleton with PAM inflated to $70 \mathrm{kPa}$ for participant 1

With reference to Figure 5.5, the PAM stretched no more than $2 \mathrm{~cm}$ during the gait cycle. This was much lower than what was expected. However, it is in accordance with the reduced angular rotation of the ankle joint.

Figures 5.4 and 5.5 demonstrated that the exoskeleton with the PAM inflated to 70 $\mathrm{kPa}$ reduced the range of motion of the ankle for participant 1 . The remaining four participants demonstrated a similar result. Table 5.1 presents the average change in maximum range of motion across all participants. For instance, the ankle with the exoskeleton experienced an average reduction in total range of motion of $19.2^{\circ}$. The table also shows that the range of motion of the knee and hip joints was minimally affected. The tests with the exoskeleton all show a decrease in maximum range of motion of the ankle for the leg with the exoskeleton. Furthermore there is a relation between the stiffness of the spring element and the reduction of the angular range of motion. The stiffer spring elements caused a greater reduction in range of motion. Table 5.2 presents the average walking speed of each participant for all tests. The participants showed little variation in walking speed across the various tests. 
Table 5.1: Average variation in joint range of motion during walking trials for both legs (with and without exoskeleton)

\begin{tabular}{llllllll}
\hline & & \multicolumn{2}{c}{ Ankle } & \multicolumn{2}{c}{ Knee } & \multicolumn{2}{c}{ Hip } \\
\cline { 2 - 8 } & & Avg $\left(^{\circ}\right)$ & SD & Avg $\left(^{\circ}\right)$ & SD & Avg $\left(^{\circ}\right)$ & SD \\
\hline \hline \multirow{2}{*}{ PAM 70 kPa } & With Exo & -19.21 & -4.54 & -1.69 & -4.00 & -1.12 & -2.11 \\
\cline { 2 - 8 } & No Exo & -1.68 & -2.48 & -0.72 & -3.44 & +0.26 & -3.57 \\
\hline \multirow{2}{*}{ PAM 40 kPa } & With Exo & -17.40 & -5.19 & +0.16 & -2.40 & -1.10 & -2.26 \\
\cline { 2 - 8 } & No Exo & -1.68 & -2.56 & -0.70 & -3.34 & -0.02 & -3.10 \\
\hline \multirow{2}{*}{ Elastic } & With Exo & -13.32 & -4.27 & -0.69 & -3.56 & -1.48 & -2.14 \\
\cline { 2 - 8 } & No Exo & -2.08 & -2.39 & -0.74 & -2.60 & -0.10 & -2.64 \\
\hline \multirow{2}{*}{ No Spring } & With Exo & -8.54 & -3.57 & -0.97 & -2.20 & -1.34 & -2.40 \\
\cline { 2 - 8 } & No Exo & -1.04 & -2.20 & -0.82 & -2.47 & -0.22 & -1.59 \\
\hline
\end{tabular}

Table 5.2: Average walking speed during human testing for participants 1-5. Speeds presented in $\mathrm{m} / \mathrm{s}$

\begin{tabular}{cccccccccccc}
\hline & \multicolumn{3}{c}{ Baseline } & \multicolumn{2}{c}{ PAM $70 \mathrm{kPa}$} & \multicolumn{2}{c}{ PAM $40 \mathrm{kPa}$} & \multicolumn{2}{c}{ Elastic } & \multicolumn{2}{c}{ No Spring } \\
\cline { 2 - 11 } & Avg & SD & Avg & SD & Avg & SD & Avg & SD & Avg & SD \\
\hline \hline 1 & 1.13 & 0.042 & 1.18 & 0.034 & 1.15 & 0.037 & 1.13 & 0.030 & 1.19 & 0.016 \\
\hline 2 & 1.28 & 0.036 & 1.28 & 0.045 & 1.33 & 0.046 & 1.32 & 0.029 & 1.28 & 0.044 \\
\hline 3 & 0.95 & 0.018 & 1.02 & 0.019 & 1.00 & 0.018 & 0.96 & 0.021 & 0.97 & 0.019 \\
\hline 4 & 1.11 & 0.036 & 1.10 & 0.036 & 1.07 & 0.022 & 1.07 & 0.044 & 1.06 & 0.041 \\
\hline 5 & 1.45 & 0.049 & 1.26 & 0.030 & 1.29 & 0.059 & 1.32 & 0.033 & 1.35 & 0.021 \\
\hline Avg & 1.18 & 0.19 & 1.17 & 0.11 & 1.17 & 0.14 & 1.16 & 0.16 & 1.17 & 0.16 \\
\hline
\end{tabular}

The kinematic results presented in this section, show that the stiffer PAM significantly reduced the angular variation of the ankle joint. However, the tests using lower stiffness spring elements displayed a smaller reduction in angular range of motion. That being said, the results indicate that the exoskeleton reduced the maximum range of motion of the ankle joint by at least $8.5^{\circ}$ regardless of the spring element being used. However, despite the reduction in joint angle, the walking speed was minimally affected by the various exoskeleton tests. 


\subsubsection{EMG Results}

The processed EMG results are presented in this section. Similar to the kinematic results presented in Section 5.3.1, this section begins by presenting the normal walking pattern, to which, all other results are compared.

Figure 5.6 shows the output of the processed EMG signal for the normal walking test for participant 1 .
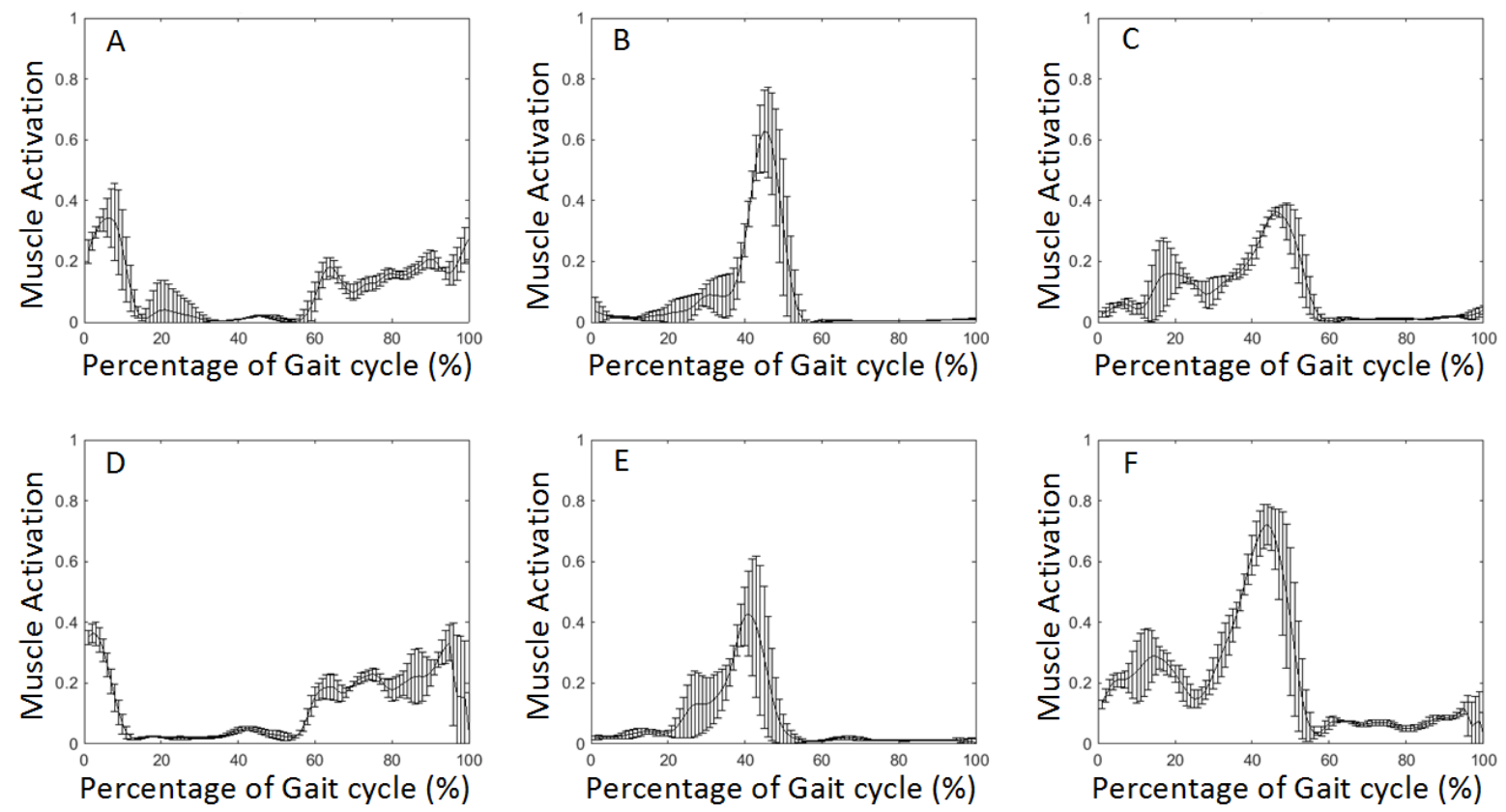

Figure 5.6: EMG output of the baseline walking trial. The image shows the recorded muscle activation of the six specific muscles during the gait cycle. A) Right Tib Ant, B) Right Gas Med, C) Right Soleus, D) Left Tib Ant, E) Left Gas Med, F) Left Soleus

With reference to Figure 5.6, the baseline data is what would be expected. For instance, at heel strike ( $0 \%$ on the $X$ axis), The Tibialis Anterior (T.A.) should be active to lift the toes off of the ground so that the heel touches the ground before the rest of the foot. In graph A), of Figure 5.6, the Tibialis Anterior muscles are active during this phase of the gait. As the toes fall and the entire foot makes contact with the floor, the T.A. should go silent, which it does. Then the calf muscles (both the Soleus and Gastrocnemius Medialis) should contract slightly as the Achilles tendon begins to stretch. Following this, there should be a large spike in activation of the calves followed by an abrupt drop to zero. The foot has pushed off the ground and is traveling through the 
air towards the next heel strike. Once again the T.A. should be active to prepare the foot for heel contact. From these the muscle activation observations it was concluded that the EMG sensors were working properly.

The results for participant 1 for the test using the PAM at $70 \mathrm{kPa}$ are presented in Figure 5.7. The general shape of the muscle activation graphs is similar to the baseline case, Figure 5.6. This indicates that the use of the exoskeleton did not significantly alter the walking pattern of the participant.
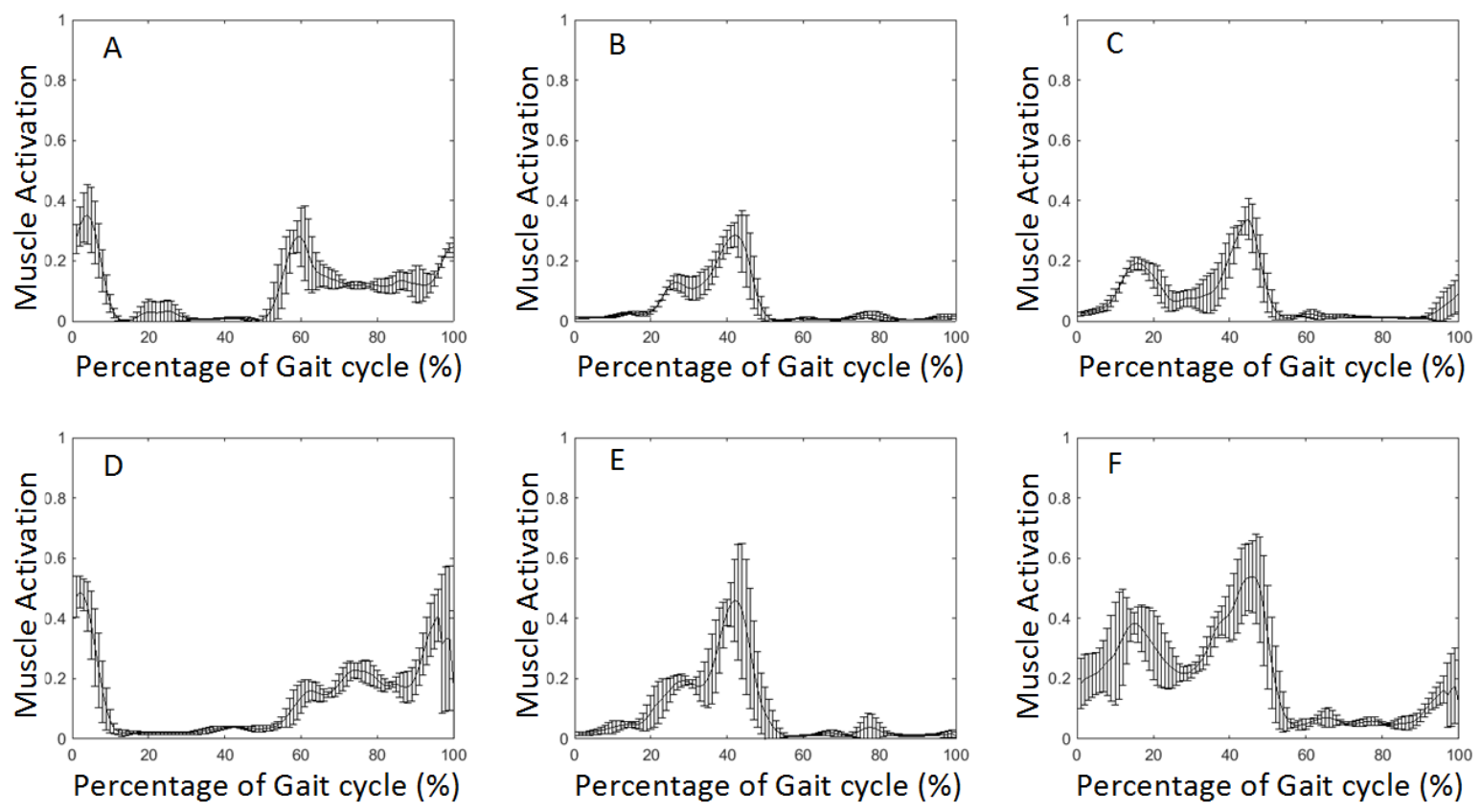

Figure 5.7: EMG output of the walking trial with the exoskeleton and the PAM inflated to $70 \mathrm{kPa}$. The image shows the recorded muscle activation of the six specific muscles during the gait cycle. A) Right Tib Ant, B) Right Gas Med, C) Right Soleus, D) Left Tib Ant, E) Left Gas Med, F) Left Soleus

The exoskeleton was also tested with a PAM inflated to $40 \mathrm{kPa}$. The results are presented in Figure 5.8. 

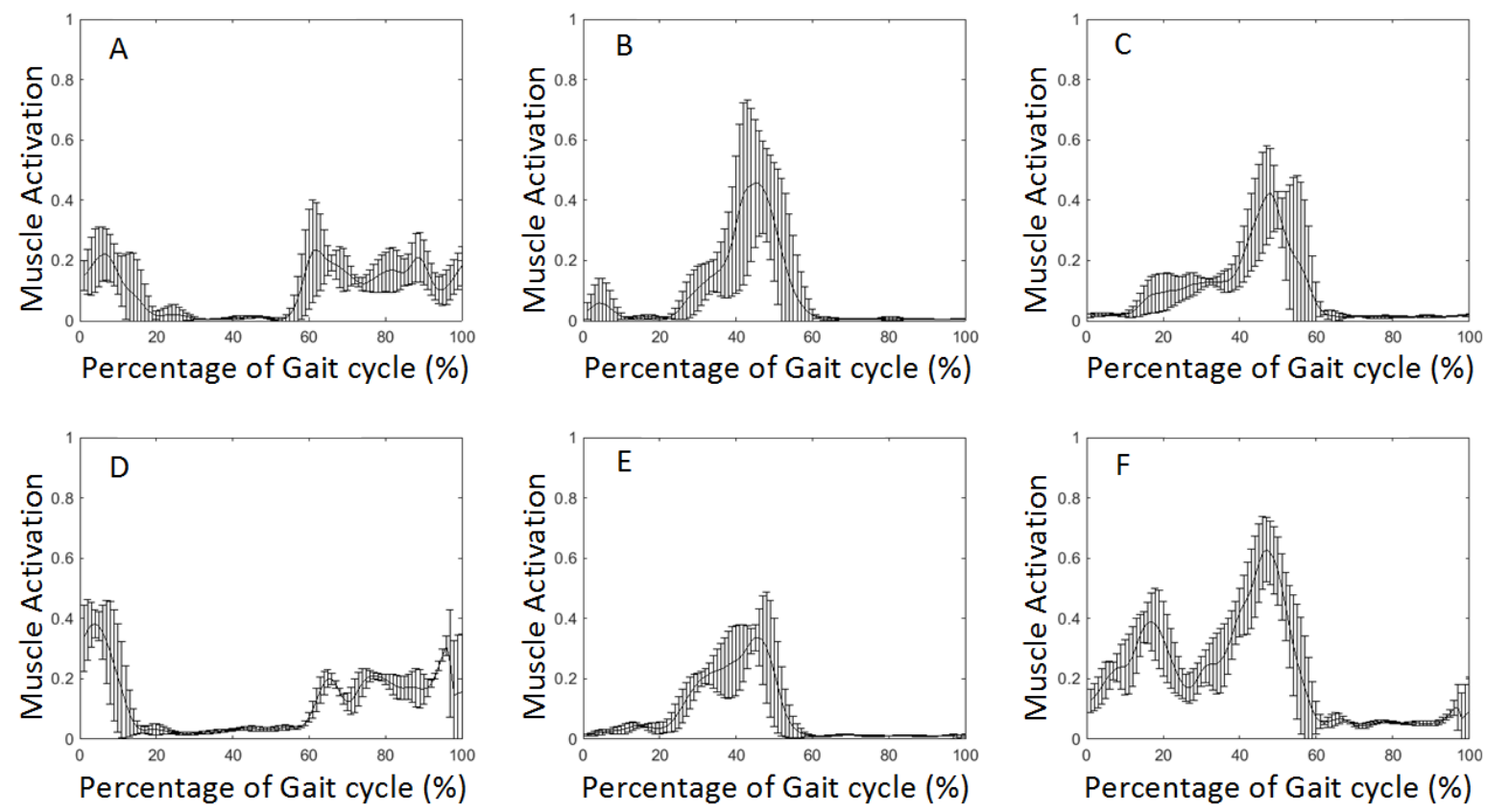

Figure 5.8: EMG output of the walking trial with the exoskeleton and the PAM inflated to $40 \mathrm{kPa}$. The image shows the recorded muscle activation of the six specific muscles during the gait cycle. A) Right Tib Ant, B) Right Gas Med, C) Right Soleus,

D) Left Tib Ant, E) Left Gas Med, F) Left Soleus

The graphs in Figure 5.8 illustrate that the variation between takes is very apparent in the right G.M. muscle (Graph B). The magnitude of the right G.M. is also higher than the other PAM test, Figure 5.7. Otherwise, the results are very similar to the case with the $70 \mathrm{kPa}$ PAM.

To directly compare the maximum muscle contraction amplitudes, the various tests have been plotted together, the complete results for participant 1 are found in Appendix E. The muscular activation of the Gastrocnemius Medialis for the right and left legs is presented in Figures 5.9 and 5.10. 


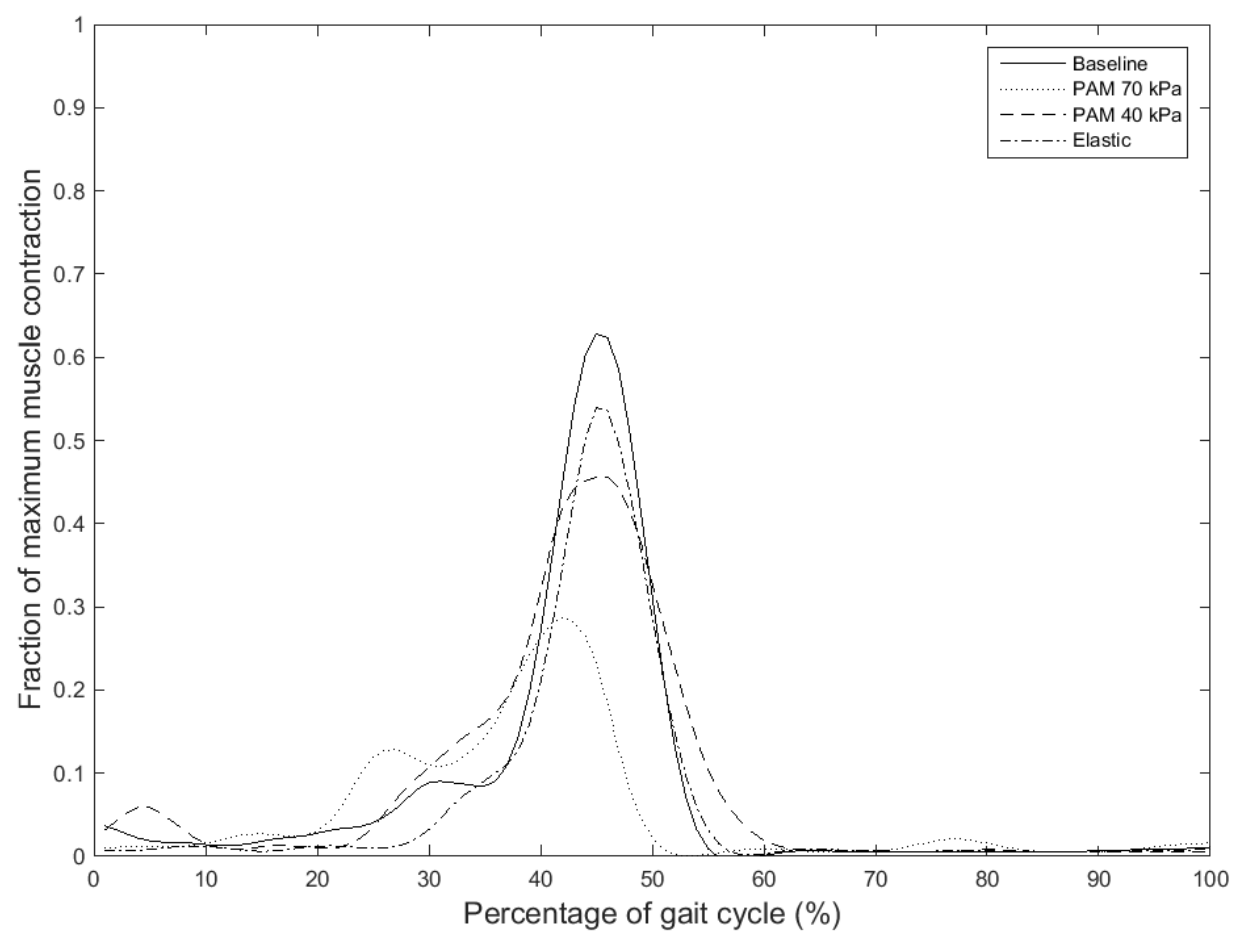

Figure 5.9: Comparison of the muscle activation in the Right Gastrocnemius Medialis during the PAM $70 \mathrm{kPa}$, PAM $40 \mathrm{kPa}$, Elastic, and baseline trials for subject 1. The exoskeleton was worn on the Right leg 


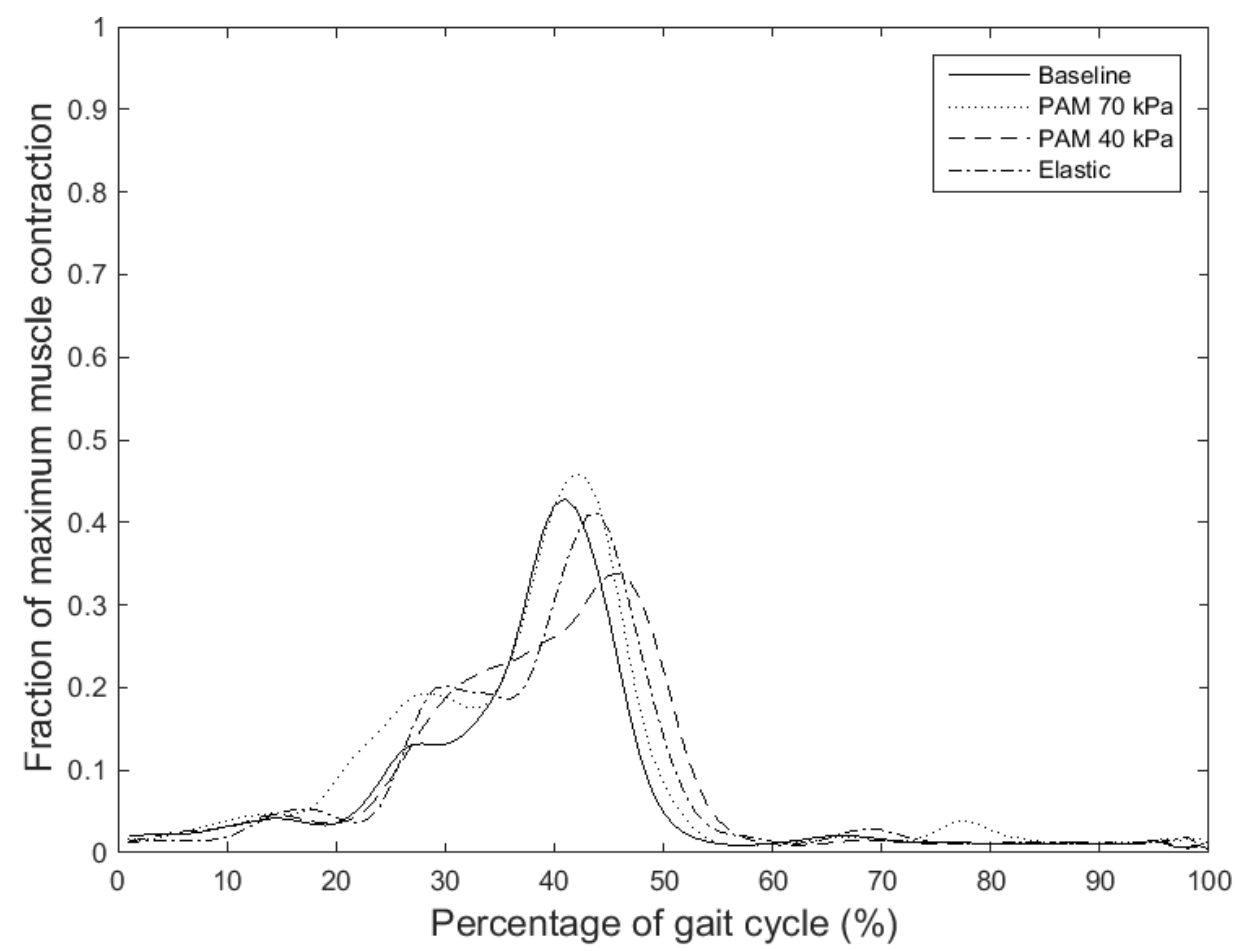

Figure 5.10: Comparison of the muscle activation in the Left Gastrocnemius Medialis during the PAM $70 \mathrm{kPa}$, PAM $40 \mathrm{kPa}$, Elastic, and baseline trials for subject 1 . The exoskeleton was worn on the Right leg

With reference to Figure 5.9, it can be seen that the muscular activation of the right calf is decreased relative to the baseline for participant 1 . Furthermore, the stiffness of the spring directly relates to the magnitude of the decrease, with the PAM inflated to $70 \mathrm{kPa}$ causing the largest decrease in muscle activation.

The muscle activation of the left leg, as presented in Figure 5.10 shows a much less distinct difference in the amplitude between tests. The maximum amplitude attained by the tests with the PAM at $70 \mathrm{kPa}$ and the Elastic band are very near to the magnitude obtained during the baseline trial. The trial with the PAM inflated to 40 $\mathrm{kPa}$ shows a lower magnitude than the other trials. However, in all, the variation present between the various tests is much less than the right leg. More importantly, the use of the exoskeleton does not significantly increase the activation of the Gastrocnemius Medialis muscle on the opposing leg.

The EMG results appear to indicate that the exoskeleton is able to reduce the activation of the calf muscles without significantly altering the gait patterns of the user or increasing the muscle activation of the other leg. However this result is called into question when the total muscle activation is computed over the entire gait cycle and 
averaged over all 5 participants. Table 5.3 presents the variation of muscle activation when compared to the baseline of each participant. These values were then averaged between participants. The positive values indicate that the muscular activation was greater than the baseline. The negative sign indicates that the muscular activation decreased relative to the baseline.

Table 5.3: Average muscle activation values when compared to baseline for both legs (with and without exoskeleton)

\begin{tabular}{lllccccc}
\hline & \multicolumn{2}{c}{ Tib. Ant. (\%) } & \multicolumn{2}{c}{ Gas. Med. (\%) } & \multicolumn{2}{c}{ Soleus (\%) } \\
\cline { 2 - 8 } & & Avg & SD & Avg & SD & Avg & SD \\
\hline \multirow{2}{*}{ PAM 70 kPa } & With Exo & 11.34 & 39.99 & -0.58 & 16.39 & -21.38 & 40.18 \\
\cline { 2 - 8 } & No Exo & -7.25 & 46.08 & 23.14 & 19.88 & 29.38 & 29.24 \\
\hline \multirow{2}{*}{ PAM 40 kPa } & With Exo & 4.10 & 34.38 & 7.83 & 10.26 & -21.47 & 39.23 \\
\cline { 2 - 8 } & No Exo & -14.41 & 41.41 & 17.83 & 10.58 & 21.25 & 21.34 \\
\hline \multirow{2}{*}{ Elastic } & With Exo & 22.18 & 24.09 & 3.90 & 23.29 & -10.05 & 9.58 \\
\hline \multirow{2}{*}{ No Spring } & No Exo & -0.66 & 9.00 & 3.46 & 14.20 & 8.24 & 9.73 \\
\cline { 2 - 8 } & With Exo & 16.70 & 22.54 & 2.03 & 18.92 & -2.94 & 17.24 \\
\hline & No Exo & 0.02 & 9.21 & 14.84 & 7.52 & 9.45 & 8.06 \\
\hline
\end{tabular}

The average muscle activation results indicate that for the case of the PAM at 70 $\mathrm{kPa}$, the Gas. Med. was less active during the entire gait cycle. However, the Tibialis Anterior on the leg with the exoskeleton shows increased activation while the leg without the exoskeleton shows decreased activation. Furthermore, Table 5.3 illustrates that the average activation of the Tibialis Anterior varies significantly when compared to the baseline trial. For instance, for the test using the PAM at $40 \mathrm{kPa}$, the leg with the exoskeleton showed an increase of $4.1 \%$, and the opposite leg, a decrease of $14.4 \%$. Therefore, when walking with the exoskeleton, the activation of the Tibialis Anterior of the leg with the exoskeleton increased while that of the intact leg decreased. This may be due to the decrease in angular range of motion cause by the exoskeleton. Moreover, the results indicate that the activation of the Soleus decreased with the use of the exoskeleton, even the No Spring test. This indicates that the so called "worst case scenario" in which the exoskeleton was worn but not assistance was provided actually reduced the muscular activation of the Soleus. 


\subsection{Limitations and Sources of Error}

\subsubsection{Limitations of the Exoskeleton Device}

Through the human testing it became apparent that there were two major flaws with the mechanical device. These issues are closely related and contributed to one another, first, the fixation of the device to the user, and second the timing mechanism.

The device was intended to be worn over top of the shoes of the user which led to problems. By placing the device over the shoe there is a layer of material that separates the device and the biological limb. Therefore, there is opportunity for energy loss or relative movement as forces are transmitted from the device to the user and vice versa. Placing the device over the shoe, as opposed to moulding an insole, also increases the amount of straps and fixation systems required. Since the timing mechanism relies on the angle of the ankle joint, the play induced by the relative movement between the device and the user reduces the range of angles experienced by the timing mechanism.

The timing mechanism is activated based on the rotation of the ankle. To move from the engaged to disengaged position the engagement arm has to slide from one end of the pawl to the other. For this to occur, the ankle must rotate by $7^{\circ}$. During this rotation the mechanism is unable to transmit a torque and thus the spring cannot be loaded. Therefore the rotation of the joint while the device is switching states is essentially wasted. When the ankle goes through the gait cycle, the maximum variation of the ankle angle is approximately $35^{\circ}$. During the testing, it became apparent that the engagement and disengagement could not be reliably achieved without exaggerating the movements of the foot. This is likely due to the fact that the timing mechanism required a large angular rotation to function $\left(7^{\circ}\right)$ combined with the fact that the relative movement between the device and the user reduced the angular rotation achieved by the device.

\subsubsection{Limitations of Methodology and testing protocols}

The most significant limitation present in the human testing protocol is the insufficient training of the participants. Like any device or tool, it takes time to become proficient and take full advantage of it during use. In the case of exoskeletons and 
walking assist devices, this is no different. The present testing methodology allowed approximately 10 minutes for the user to get used to the device. The unfamiliarity of the participants may be the cause of the decrease in angular range of motion of the ankle joint. 


\section{Chapter 6: Conclusions and Recommendations}

This thesis is divided into three main sections, the PAM modelling and testing, the Exoskeleton design, fabrication and mechanical testing, and finally the human testing. This chapter summarizes the results of each section before describing the direction of future work. 


\subsection{Conclusion}

A PAM model was first developed to simulate the distinctive motion that the PAM undergoes when used as a passive spring element. The PAM modelling was based on a previously developed analytical model to which two components were added. A polytropic assumption was added to the gas pressure and temperature model. Furthermore, the bladder material was tested biaxially to characterize the stress in both the longitudinal and circumferential directions. Once these two additions were incorporated into the model it was translated into Matlab and simulated. The model was validated using experimental testing. The force and pressure results showed that at high pressures the PAM matched the isothermal case very well. However, at low pressure the simulated values underestimated the experimental values. At these low pressures the addition of the bladder stress model improved the performance of the force simulation but did not affect the pressure variation. The temperature measurements revealed a variation of less than $1^{\circ} \mathrm{C}$ during the elongation cycle. This result indicates that an isothermal model is valid. This is further supported by the pressure values obtained from the high pressure tests which also fit the isothermal case best.

An ankle exoskeleton was designed using (PAM) placed in parallel with the Achilles tendon. The device included a custom ratchet and pawl mechanism to engage and disengage the PAM so that it would assist the user during stance phase but not hinder the user during the swing phase. The mechanical testing demonstrated that the PAM inflated to $70 \mathrm{kPa}$ showed an adequate $25 \%$ replication of the natural ankle torque when only partially stretched. If the exoskeleton moves through the complete range of motion the torque produced by the exoskeleton would be much higher than required.

A prototype exoskeleton was fabricated out of aluminum and 3D printed components. The custom clutch mechanism was built and mechanically functioned as intended. The engagement and disengagement timing could be adjusted using two screws. To examine the exoskeletons ability to assist the user, human walking trials were conducted. Electromyography was used to examine the muscular activation of the Tibialis Anterior, Gastrocnemius Medialis and Soleus muscles. The EMG results were difficult to interpret and indicated that the activation of the Soleus was decreased on the leg with the exoskeleton for all tests. This combined with the reduction in angular range of motion of the ankle seems to indicate that the exoskeleton 
altered the walking pattern of the wearer. Thus, the EMG results cannot truly be directly compared.

\subsection{Future Work}

One possible improvement of the PAM model and experimental testing presented in this work, is the slow elongation rates during testing. The use of PAM in walking assist devices implies a certain speed of elongation. However, due to the limitations of the machine used, this was not reflected in the current testing. In the future, it would be valuable to compare the effect that various elongation rates have on the pressure, force and most of all, the temperature variation of the PAM.

The clutch mechanism of the exoskeleton was intended to engage the PAM during the stance phase then disengage it during the swing phase. In human testing this did not happen. A combination of the device not following the user, and the fact that the timing mechanism required a large angular deviation, meant that the device remained engaged. In the future the device would be modified so that the timing mechanism could transitions more easily between engaged and disengaged states. Furthermore, the attachment of the device to the user would need to be re-designed to reduce the relative movement between the device and the user.

The human testing did not allow sufficient time for the participant to get used to the device between tests. In future testing the participant will be given more time to adapt between tests. Furthermore, additional human subjects as well as statistical methods should be included to increase the value of the study.

Future testing should also use an adequate gait lab with operational force plates to acquire kinetic data and precisely identify gait phases (e.g. heel strike). This would improve the accuracy of the data as well as reduce the required processing time. 


\section{References}

[1] R. Dodel and A. Schrag. Health-Related Quality of Life in Movement Disorders. In Victor R. Preedy and Ronald R. Watson, editors, Handbook of Disease Burdens and Quality of Life Measures, pages 4013-4034. Springer New York, 2010.

[2] R. T. Staff. Rewalk, 'FDA Approves ReWalk Robotics',2014. [Online]. Available :http:/ /www.roboticstrends.com/article/. [Accessed: 5 June 2017].

[3] S. Crowe, 'Honda Walking Assist Device Going on Sale', 2015. [Online]. Available:http://www.roboticstrends.com/article/. [Accessed: 5 June 2017].

[4] K. M. Lee and D. Wang. Design analysis of a passive weight-support lowerextremity-exoskeleton with compliant knee-joint. In 2015 IEEE International Conference on Robotics and Automation (ICRA), pages 5572-5577, 2015.

[5] W. van Dijk and H. Van der Kooij. XPED2: A Passive Exoskeleton with Artificial Tendons. IEEE Robotics Automation Magazine, 21(4):56-61, 2014.

[6] Michael W. Whittle. Chapter 2 - Normal gait. In Gait Analysis (Fourth Edition), pages 47-100. Butterworth-Heinemann, 2007.

[7] R. Unal, R. Carloni, E. G. Hekman, S. Stramigioli, and H. M. Koopman. Biomechanical conceptual design of a passive transfemoral prosthesis. Conf Proc IEEE Eng Med Biol Soc, 2010:515-518, 2010.

[8] Steven H. Collins, M. Bruce Wiggin, and Gregory S. Sawicki. Reducing the energy cost of human walking using an unpowered exoskeleton. Nature, 522(7555):212-215, 2015.

[9] James A. Norris, Kevin P. Granata, Melanie R. Mitros, Erica M. Byrne, and Anthony P. Marsh. Effect of augmented plantarflexion power on preferred walking speed and economy in young and older adults. Gait $\mathcal{E}$ Posture, 25(4):620-627, 2007. 
[10] Gregory S. Sawicki and Daniel P. Ferris. Mechanics and energetics of level walking with powered ankle exoskeletons. Journal of Experimental Biology, 211(9):1402-1413, 2008.

[11] Justin Leclair. Development of a Passive Ankle Exoskeleton (PAXO) to Reduce Joint Load when Walking using a Pneumatic Artificial Muscle Based on an Analytical Stiffness Model M.S. Thesis Department of Mechanical Engineering, University of Ottawa, Ontario, Canada, 2016.

[12] M. Doumit J. Murillo 'JMBE-Design and Evaluation of Pneumatic Artificial Muscle for Powered Transfemoral Prostheses', Journal of Medical and Biological Engineering, 2014.

[13] Steven H. Collins, M. Bruce Wiggin, and Gregory S. Sawicki. Reducing the energy cost of human walking using an unpowered exoskeleton. Nature, 522(7555):212-215, 2015.

[14] Ana Cristina de David, Felipe Pivetta Carpes, and Darren Stefanyshyn. Effects of changing speed on knee and ankle joint load during walking and running. Journal of Sports Sciences, 33(4):391-397, 2015.

[15] Fisch et al. Buesing, Carolyn. Effects of a wearable exoskeleton stride management assist system (SMA®) on spatiotemporal gait characteristics in individuals after stroke: A randomized controlled trial. Journal of NeuroEngineering and Rehabilitation, 12:69, 2015.

[16] Ryosuke Kitatani, Koji Ohata, Hideaki Takahashi, Saori Shibuta, Yu Hashiguchi, and Natsuki Yamakami. Reduction in Energy Expenditure During Walking Using an Automated Stride Assistance Device in Healthy Young Adults. Archives of Physical Medicine and Rehabilitation, 95(11):2128-2133, 2014.

[17] Honda Worldwide Honda Bodyweight Support Assist Device Selected for Innovation Exhibit at Smithsonian's CooperHewitt, National Design Museum, 2010. [Online]. Available : http://world.honda.com/news/2010/c100512bodyweight-support-assistdevice/. [Accessed : 5 November 2015].

[18] 'ReWalk 6.0 Home', [Online]. Available :http://rewalk.com/. [Accessed: 5 June 2017]. 
[19] Gregory S. Sawicki and Daniel P. Ferris. Mechanics and energetics of incline walking with robotic ankle exoskeletons. Journal of Experimental Biology, 212(1):32-41, 2009.

[20] Steven H. Collins and Arthur D. Kuo. Recycling energy to restore impaired ankle function during human walking. PLoS ONE, 5(2):e9307, 2010.

[21] Ava D. Zelik et al. Segal. The effects of a controlled energy storage and return prototype prosthetic foot on transtibial amputee ambulation. Human Movement Science, 31(4):918-931, 2012.

[22] Keith E. Gordon and Daniel P. Ferris. Learning to walk with a robotic ankle exoskeleton. Journal of Biomechanics, 40(12):2636-2644, 2007.

[23] P. Cherelle, A. Matthys, V. Grosu, B. Vanderborght, and D. Lefeber. The AMPFoot 2.0: Mimicking intact ankle behavior with a powered transtibial prosthesis. In 2012 4th IEEE RAS EMBS International Conference on Biomedical Robotics and Biomechatronics (BioRob), pages 544-549, 2012.

[24] P. Cherelle, V. Grosu, A. Matthys, B. Vanderborght, and D. Lefeber. Design and Validation of the Ankle Mimicking Prosthetic (AMP-) Foot 2.0. IEEE Transactions on Neural Systems and Rehabilitation Engineering, 22(1):138-148, 2014.

[25] G. A. Lichtwark and A. M. Wilson. Interactions between the human gastrocnemius muscle and the Achilles tendon during incline, level and decline locomotion. J. Exp. Biol., 209:4379-4388, 2006.

[26] T. Fukunaga, K. Kubo, Y. Kawakami, S. Fukashiro, H. Kanehisa, and C. N. Maganaris. In vivo behaviour of human muscle tendon during walking. Proc Biol Sci, 268(1464):229-233, 2001.

[27] L.M. Mooney, C.H. Lai, and E.J. Rouse. Design and characterization of a biologically inspired quasi-passive prosthetic ankle-foot. In 2014 36th Annual International Conference of the IEEE Engineering in Medicine and Biology Society, EMBC 2014, pages 1611-1617.

[28] Zahra Safaeepour, Ali Esteki, Farhad Tabatabai Ghomshe, and Noor Azuan Abu Osman. Quantitative analysis of human ankle characteristics at different gait phases and speeds for utilizing in ankle-foot prosthetic design. Biomed Eng Online, 13:19, 2014. 
[29] Ottobock, 'Thermoplastic AFO', [Online]. Available :https:// professionals.ottobockus.com/. [Accessed: 5 June 2017].

[30] D.J.J. Bregman, Groot De, Diggele Van, H. Meulman, H. Houdijk, and J. Harlaar. Polypropylene ankle foot orthoses to overcome drop-foot gait in central neurological patients: A mechanical and functional evaluation. Prosthetics and Orthotics International, 34(3):293-304, 2010.

[31] H. Gök, A. Küçükdeveci, H. Altinkaynak, G. Yavuzer, and S. Ergin. Effects of ankle-foot orthoses on hemiparetic gait. Clinical Rehabilitation, 17(2):137-139, 2003.

[32] Seyoung Kim, Youngsu Son, Sangkyu Choi, Sangyong Ham, and Cheolhoon Park. Design of a simple, lightweight, passive-elastic ankle exoskeleton supporting ankle joint stiffness. Review of Scientific Instruments, 86(9):095107, 2015.

[33] M.B. Wiggin, G.S. Sawicki, and S.H. Collins. An exoskeleton using controlled energy storage and release to aid ankle propulsion. In 2011 IEEE International Conference on Rehabilitation Robotics (ICORR), pages 1-5, 2011.

[34] Zahra Safaeepour, Ali Esteki, Farhad Tabatabai Ghomshe, and Noor Azuan Abu Osman. Quantitative analysis of human ankle characteristics at different gait phases and speeds for utilizing in ankle-foot prosthetic design. Biomed Eng Online, 13:19, 2014.

[35] M. Doumit, A. Fahim, and M. Munro. Analytical Modeling and Experimental Validation of the Braided Pneumatic Muscle. IEEE Transactions on Robotics, 25(6):1282-1291, 2009.

[36] Daniel P. Ferris, Joseph M. Czerniecki, and Blake Hannaford. An Ankle-Foot Orthosis Powered by Artificial Pneumatic Muscles. Jornal of Applied Biomechanics, 21(2):189-197, 2005.

[37] Gyoosuk Kim, Shinill Kang, Hyeonseok Cho, Jeicheong Ryu, Museoung Mun, and Kyunghoon Kim. Modeling and simulation of powered hip orthosis by pneumatic actuators. Int. J. Control Autom. Syst., 8(1):59-66, 2010.

[38] Gaylord, Richard H, Fluid Actuated Motor System and Stroking Device, US Patent US2844126 A. U.S. Classification 92/90, 138/30; International Classification F15B15/10, 1958. 
[39] S. Thongchai, M. Goldfarb, N. Sarkar, and K. Kawamura. A frequency modeling method of rubbertuators for control application in an IMA framework. In American Control Conference, 2001. Proceedings of the 2001, volume 2, pages 1710-1714 vol.2.

[40] Characteristics of the braided fluid actuator [by] H.F. Schulte, Jr., D.F. Adamski [and] J.R. Pearson.

[41] Ching-Ping Chou and B. Hannaford. Measurement and modeling of McKibben pneumatic artificial muscles. IEEE Transactions on Robotics and Automation, 12(1):90-102, 1996.

[42] B. Tondu and P. Lopez. Modeling and control of McKibben artificial muscle robot actuators. IEEE Control Systems, 20(2):15-38, 2000.

[43] B. S. Kang, C. S. Kothera, B. K. S. Woods, and N. M. Wereley. Dynamic modeling of Mckibben pneumatic artificial muscles for antagonistic actuation. In IEEE International Conference on Robotics and Automation, 2009. ICRA '09, pages 182187, 2009.

[44] D. B. Reynolds, D. W. Repperger, C. A. Phillips, and G. Bandry. Modeling the Dynamic Characteristics of Pneumatic Muscle. Annals of Biomedical Engineering, 31(3):310-317, 2003.

[45] Jinghui Cao, Sheng Quan Xie, Mingming Zhang, and Raj Das. A New Dynamic Modelling Algorithm for Pneumatic Muscle Actuators. In Xianmin Zhang, Honghai Liu, Zhong Chen, and Nianfeng Wang, editors, Intelligent Robotics and Applications, number 8918 in Lecture Notes in Computer Science, pages 432-440. Springer International Publishing.

[46] T. Itto and K. Kogiso. Hybrid modeling of McKibben pneumatic artificial muscle systems. In 2011 IEEE International Conference on Industrial Technology (ICIT), pages 65-70, 2011.

[47] K. Kogiso, K. Sawano, T. Itto, and K. Sugimoto. Identification procedure for McKibben pneumatic artificial muscle systems. In 2012 IEEE/RSJ International Conference on Intelligent Robots and Systems, pages 3714-3721, 2012.

[48] J. Boržiková, J. Pitel, M. Tóthová, and B. Šulc. Dynamic simulation model of PAM based antagonistic actuator. In Carpathian Control Conference (ICCC), 2011 12th International, pages 28-31, 2011. 
[49] M. Tóthová and J. Pitel. Simulation of actuator dynamics based on geometric model of pneumatic artificial muscle. In 2013 IEEE 11th International Symposium on Intelligent Systems and Informatics (SISY), pages 233-237, 2013.

[50] J. Pitel' and M. Tóthová. Dynamics of pneumatic muscle actuator: Measurement and modeling. In Control Conference (ICCC), 2014 15th International Carpathian, pages 432-436, 2014.

[51] Francesco Sorge. Dynamical behaviour of pneumatic artificial muscles. Meccanica, 50(5):1371-1386, 2014.

[52] T. Vo-Minh, T. Tjahjowidodo, H. Ramon, and H. Van Brussel. A New Approach to Modeling Hysteresis in a Pneumatic Artificial Muscle Using The Maxwell-Slip Model. IEEE/ASME Transactions on Mechatronics, 16(1):177-186, 2011.

[53] 'BioTester'. [Online]. Available : http://cellscale.com/products/biotester/. [Accessed: 6 June 2017].

[54] M. Doumit and S. Pardoel. Dynamic contraction behaviour of pneumatic artificial muscle. Mechanical Systems and Signal Processing, 91:93-110, 2017.

[55] David A. Winter. Appendix A: Kinematic, Kinetic, and Energy Data. In Biomechanics and Motor Control of Human Movement, pages 296-360. John Wiley \& Sons, Inc., 2009.

[56] R. Merletti 'Welcome to SENIAM', [Online]. Available : http://www.seniam.org/. [Accessed : 8 June 2017].

[57] O. Jones, 'Anatomical Planes', 2017. [Online]. Available :http:/ / teachmeanatomy.info/the-basics/anatomical-terminology/. [Accessed: 5 June 2017].

[58] Michael W. Whittle. Chapter 1 - Basic sciences. In Gait Analysis (Fourth Edition), pages 1-45. Butterworth-Heinemann, 2007.

[59] J. M. Soucie, C. Wang, A. Forsyth, S. Funk, M. Denny, K. E. Roach, D. Boone, and Hemophilia Treatment Center Network. Range of motion measurements: Reference values and a database for comparison studies. Haemophilia, 17(3):500507, 2011.

[60] Orthopaedicsone, 'Physical Examination of the Foot and Ankle', [Online]. Available :http://www.orthopaedicsone.com/display/main/physical. [Accessed: 5 June 2017]. 
[61] O. Jones, 'muscles of the Lower Limb', 2017. [Online]. Available :http:/ / teachmeanatomy.info/lower-limb/muscles/. [Accessed: 5 June 2017].

[62] J. G. M. Beltman, M. R. van der Vliet, A. J. Sargeant, and A. de Haan. Metabolic cost of lengthening, isometric and shortening contractions in maximally stimulated rat skeletal muscle. Acta Physiol. Scand., 182(2):179-187, 2004.

[63] Masaki Ishikawa, Paavo V. Komi, Michael J. Grey, Vesa Lepola, and Gert-Peter Bruggemann. Muscle-tendon interaction and elastic energy usage in human walking. J. Appl. Physiol., 99(2):603-608, 2005.

[64] J.R. Franz, L.C. Slane, K. Rasske, and D.G. Thelen. Non-uniform in vivo deformations of the human Achilles tendon during walking. Gait and Posture, 41(1):192197, 2015.

[65] Karl E. Zelik, Tzu-Wei P. Huang, Peter G. Adamczyk, and Arthur D. Kuo. The role of series ankle elasticity in bipedal walking. Journal of Theoretical Biology, 346:75-85, 2014.

[66] J. R. Usherwood, A. J. Channon, J. P. Myatt, J. W. Rankin, and T. Y. Hubel. The human foot and heel-sole-toe walking strategy: A mechanism enabling an inverted pendular gait with low isometric muscle force. Journal of The Royal Society Interface, 2012.

[67] G. A. Lichtwark, K. Bougoulias, and A. M. Wilson. Muscle fascicle and series elastic element length changes along the length of the human gastrocnemius during walking and running. Journal of Biomechanics, 40(1):157-164, 2007.

[68] Alan Wilson and Glen Lichtwark. The anatomical arrangement of muscle and tendon enhances limb versatility and locomotor performance. Philosophical Transactions of the Royal Society of London B: Biological Sciences, 366(1570):15401553, 2011.

[69] Donald L. Bartel, Dwight T. Davy, and Tony M. Keaveny. Orthopaedic Biomechanics: Mechanics and Design in Musculoskeletal Systems. Pearson/Prentice Hall, 2006.

[70] David A. Winter. Biomechanics and Motor Control of Human Movement. John Wiley \& Sons, 2009.

[71] Chris Kirtley. Chapter 1 - The temporal-spatial parameters. In Clinical Gait Analysis, pages 15-37. Churchill Livingstone, 2006. 
[72] 'Physical Examination: Gait', 2014. [Online]. Available :http://epomedicine.com/clinical-medicine/physical-examination-gait/. [Accessed: 5 June 2017].

[73] Chris Kirtley. Chapter 5 - The ground reaction in normal gait. In Clinical Gait Analysis, pages 83-96. Churchill Livingstone, 2006.

[74] Pei-Chun Kao, Cara L. Lewis, and Daniel P. Ferris. Invariant ankle moment patterns when walking with and without a robotic ankle exoskeleton. Journal of Biomechanics, 43(2):203-209, 2010.

[75] R.C. Browning, J.R. Modica, R. Kram, and A. Goswami. The effects of adding mass to the legs on the energetics and biomechanics of walking. Medicine and Science in Sports and Exercise, 39(3):515-525, 2007.

[76] David A. Winter. The Biomechanics and Motor Control of Human Gait: Normal, Elderly and Pathological. University of Waterloo Press, 1991. 


\section{Appendix A}

\section{Human locomotion and fundamentals of gait}

\section{A.1 Human Locomotion}

Locomotion describes how an organism is able to get around. In the case of humans, this of course refers to walking. As the primary way that humans move, it has been intensively studied for decades. The human walking cycle is deceptively complex; thus any walking assist device must be very carefully designed in order to not hinder healthy gait motion.

\section{A.1.1 Physiology Anatomy of the Lower Limbs}

When analyzing human physiology, certain terminology conventions are used. Figure A.1 illustrates the three planes that are employed when discussing the human body. Namely the sagittal, transverse and coronal (frontal) planes. 


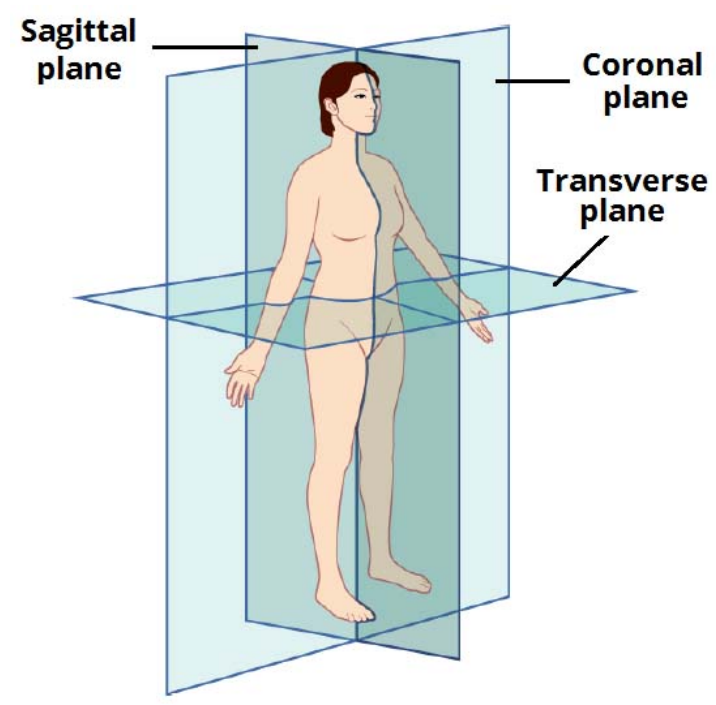

Figure A.1: Anatomical planes used to describe the human body [57]

The lower limbs are made up of three main articulations. The hip, knee and ankle joints are responsible for transferring forces to the ground and allowing specific degrees of freedom to permit locomotion. Figure A.2 illustrates the terminology describing movement at the various joints of the leg and Table A.1 quantifies the normal ranges of motion of the hip knee and ankle joints.
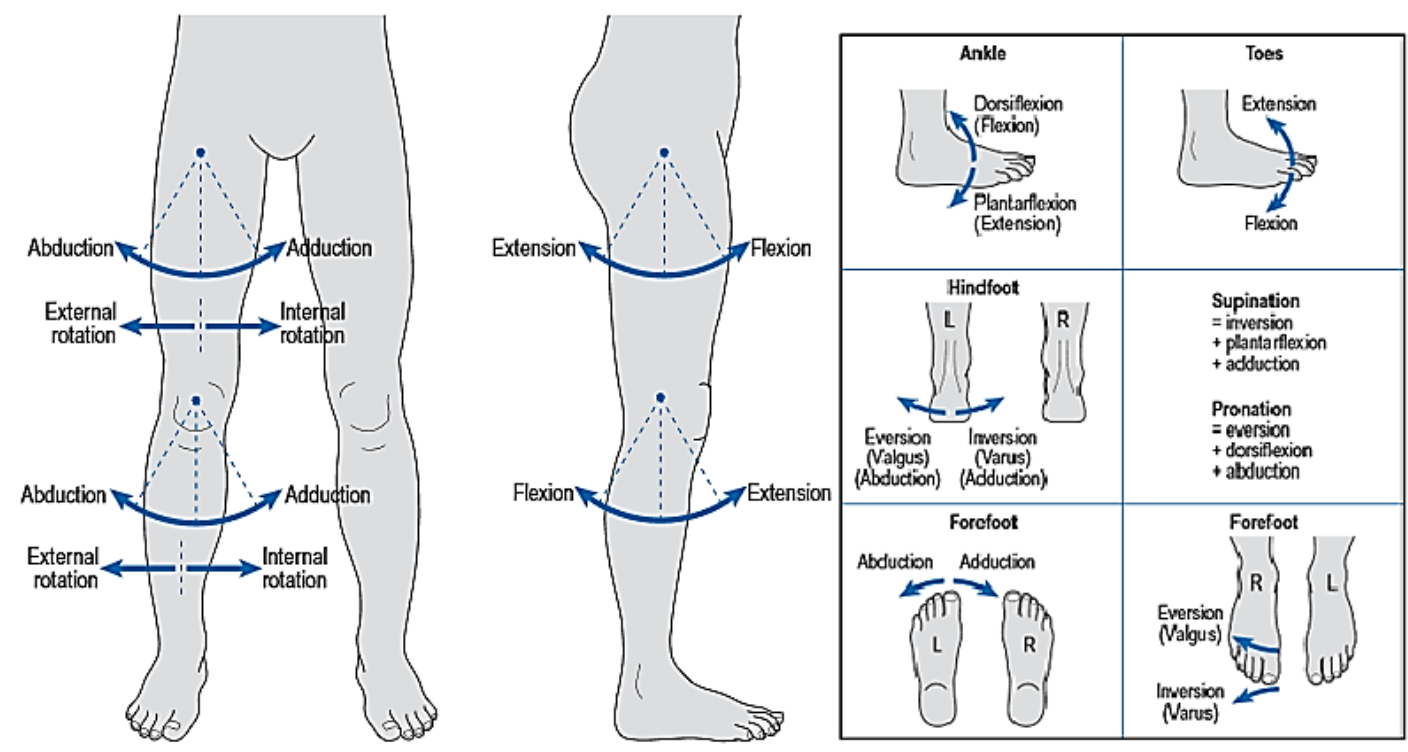

Figure A.2: Movement about the hip, knee, ankle and foot joints. Modified from [58] 
Table A.1: Table summarizing the typical Range of Motion of the lower limb joints $[11,59,60]$

\begin{tabular}{llc}
\hline Joint & Movement & Range of motion (Degrees) \\
\hline \hline \multirow{3}{*}{ Hip } & Flexion/Extension & $120 / 30$ \\
& Abduction/Adduction & $45 / 40$ \\
& Internal/External Rotation & $40 / 45$ \\
\hline \multirow{2}{*}{ Knee } & Flexion/Extension & $130 / 15$ \\
& Internal Rotation & 10 \\
\hline \multirow{3}{*}{ Ankle } & Plantar/Dorsi Flexion & $50 / 20$ \\
& Eversion/Inversion & $30 / 20$ \\
& Internal/External Rotation & $5 / 10$ \\
\hline
\end{tabular}

In order to fully grasp the complex interactions between the various muscles and soft tissues, and to design assistive devices accordingly, the anatomy of the lower limbs will be briefly introduced. Figure A.3 illustrates the muscles of the lower leg and Table A.2 their respective roles as well as the muscles considered in this work.
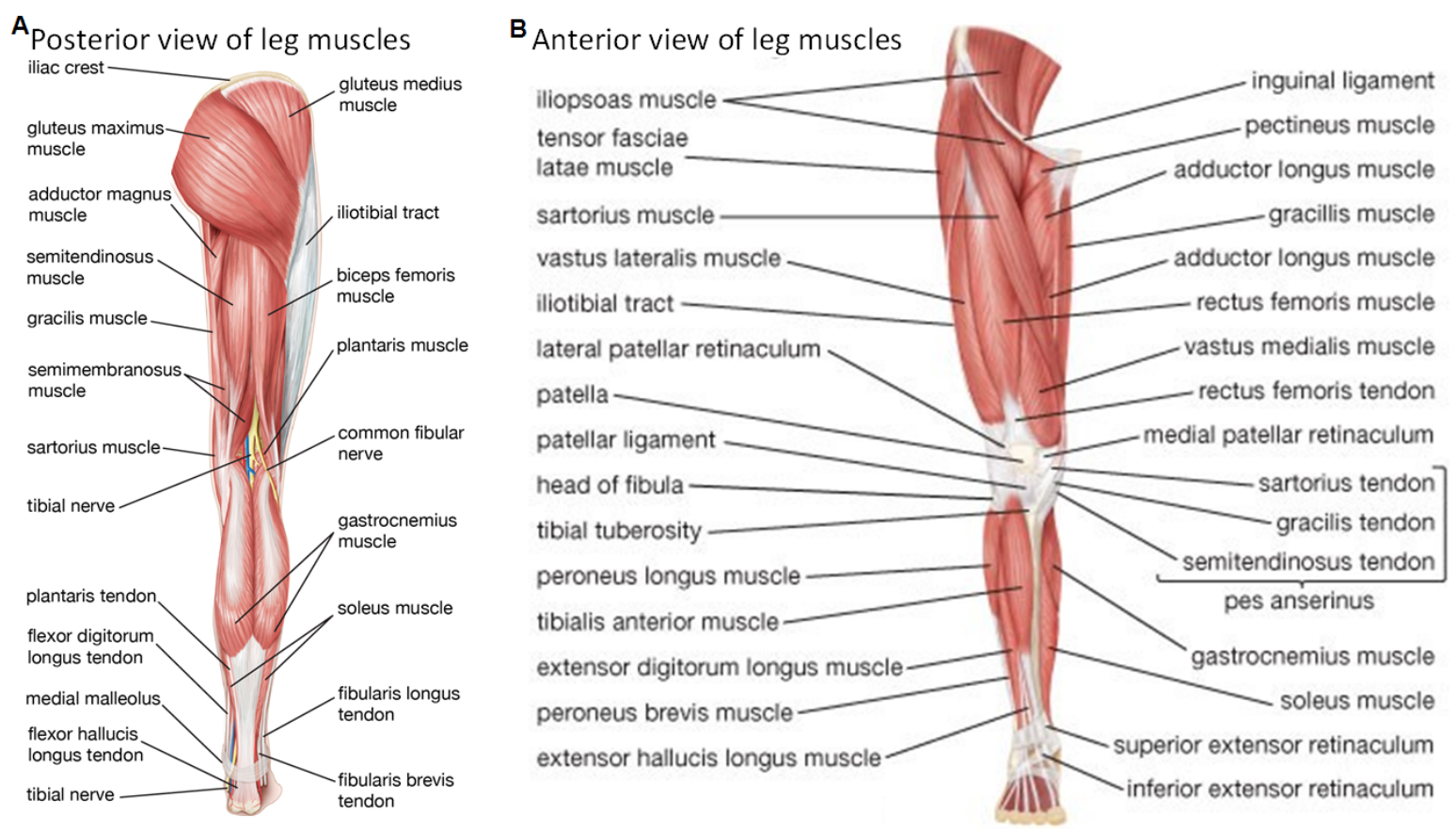

Figure A.3: General anatomy of the lower leg. A) Posterior view of leg muscles B) Anterior view of leg muscles [61] 
Table A.2: Lower limb muscles and their functions related to movements $[61,60,11]$

\begin{tabular}{llc}
\hline Joint & Movement & Muscle(s) \\
\hline \hline \multirow{3}{*}{ Hip } & Flexion & Iliopsoas \\
& Extension & Gluteus Maximus and Hamstring \\
& Abduction & Gluteus Medius and Gluteus Minimus \\
& Adduction & Adductor Longus and Adductor Magnus \\
\hline \multirow{2}{*}{ Knee } & Flexion & Hamstring \\
& Extension & Quadriceps \\
\hline \multirow{3}{*}{ Ankle } & Dorsiflexion & Tibialis Anterior \\
& Plantar Flexion & Triceps Surae \\
& Inversion & Tibialis Anterior and Tibialis Posterior \\
& Eversion & Peronei \\
\hline
\end{tabular}

Among the numerous muscles of the lower limbs, only certain pairs of muscles will be referred to in this work. Generally, the muscles that contribute to the flexion and extension of the lower limb joints are considered the most important. At the hip, the Gluteus Maximus and the Iliopsoas are examples of extensors and flexors. The knee is flexed by the Hamstrings (Biceps Femoris, Semitendinosus, Semimembranosus) and extended by the quadriceps (Rectus Femoris, Vastus Medialis, Intermedius and Lateralis). For the ankle joint, the Gastrocnemius the Soleus and the Tibialis Anterior are the primary movers. The Tibialis anterior dorsiflexes the foot and is active during the loading of the support limb immediately following initial contact. The Gastrocnemius (both medial and lateral) along with the Soleus make up what is referred to as the Triceps Surae, i.e. the calf muscles. In Chapter 6, human subjects are asked to walk while the EMG activity of their Soleus, Gastrocnemius Medialis and Tibialis Anterior muscles are monitored.

\section{Muscle Contraction}

Biological muscle tissue has three distinct modes of activation; concentric, eccentric and isometric contractions. During concentric contraction the force generated by the muscle is large enough to overcome the applied load and the muscle shortens. 
Isometric contraction, for its part, is characterized by force generation with no change in muscle length. Lastly, eccentric contraction is a muscle force that resists movement. If a muscle is generating an opposing force that slows the imposed muscle elongation, then it is said to be contracting eccentrically.

The distinction between the different modes of muscle contractions is at the base of gait cycle analysis as well as the design of assistive devices. For instance, eccentric contraction is a resistance to elongation. Therefore, the muscle in question could be emulated by the use of a mechanical component that exhibits the same physical behaviour. By that logic, using a mechanical spring-damper combination to resist the motion would assist the eccentric muscle activation and therefore reduce the metabolic cost of the individual. This is a basic concept that has been applied to almost all of the human mobility assistive devices that will be presented in this chapter.

A challenge that arises is the varied response of the muscle contraction throughout the gait cycle. A single mechanical element would likely not be able to mimic the full range of characteristics displayed by the biological tissue. The design of assistive devices is directly guided by attempting to replicate these characteristics at specific instances.

One important property of biological muscle is its energy consumption and, not all muscle contractions require the same amount of energy. Literature has shown that eccentric force production requires approximately $30 \%$ of the energy required for concentric contraction [62]. Therefore, the type of contraction will also help identify which areas of gait are the most metabolically demanding. However, the type of muscle contraction does not fully represent the energy usage within the body. The properties of the tendons and soft tissues surrounding the joint play an important role in the energetic requirements of certain movements.

\section{Muscle Tendon Unit}

The role of tendons is primarily to transmit forces between muscles and bones. Therefore, the mechanical properties of the tendon and its non-linear elasticity are important factors that determine how the forces are transferred. Tendons can be divided into two main categories, energy storing tendons and positioning tendons. The positioning tendons are stiffer and are found in joints that require precise force transfer (e.g. fingers). Energy storing tendons are able to stretch and recoil similarly to mechanical springs. The most relevant example is the Achilles tendon. During the stance 
phase of gait, the foot is flat on the ground while the body advances. This creates an elongation in the soft tissues in the posterior shank. In 2005, Ishikawa et al. [63] examined the relation between the gastrocnemius medalis, the soleus and the Achilles tendon. The use of ultrasonographic apparatuses allowed the researchers to examine the role of soft tissues in the calf. Figure A.4 illustrates the results of the study. The image shows the mechanical power in the shank throughout a single gait cycle. The three lines are the Muscle Tendon Unit (MTU), the Tendinous Tissues (TT) and the difference between the two (MTU-TT). The main finding was that the elongation of the muscle tendon unit (MTU), was due primarily to the passive stretching of the tendon (TT) and not the elongation of the muscles.

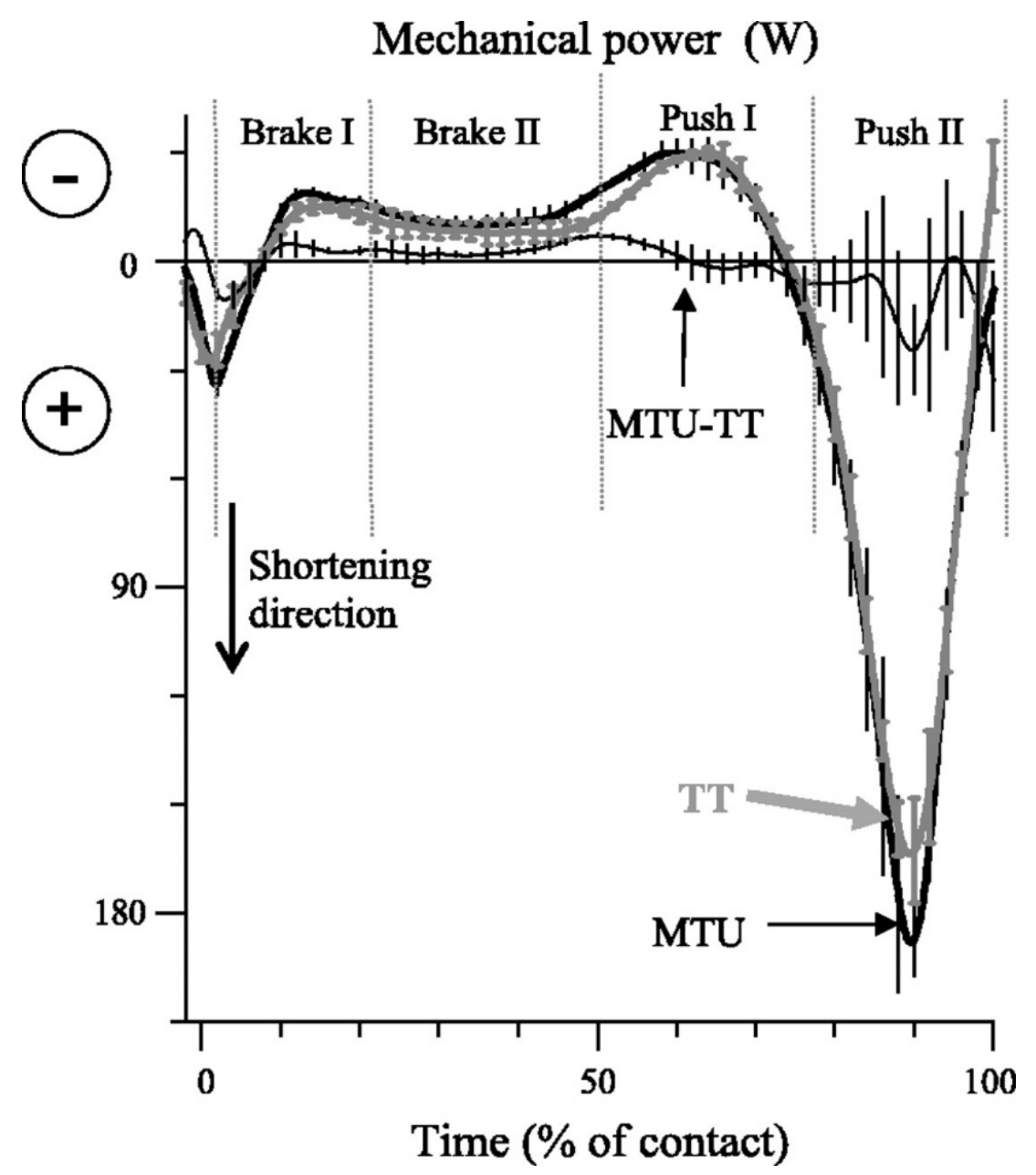

Figure A.4: Experimental results illustrating the mechanical power in the shank. MTU is the muscle tendon unit, TT is the tendinous tissues and includes aponeuroses [63]

From the work done by Ishikawa et al. [63], among others, it has been con- 
firmed that the elongation of the muscle tendon unit is largely due to the stretching of the Achilles [64], [25]. It was also discovered that during the stance phase, the gastrocnemius remained isometrically contracted and only significantly changed length during the final push off phase. Moreover, multiple studies have corroborated the finding that the tendon is responsible for a large amount of energy storage during this portion of the gait cycle $[63,65,26]$. This lead to what is commonly called the catapult behaviour of the Achilles tendon [66]. The so called catapult effect has been used to describe the series elasticity of the Achilles tendon by multiple researchers [67], [68]. These findings suggest that the calves behave very much like passive elastic components during walking. In fact, as will be presented in Section 2.1, there is currently only one passive walking assist device that is able to reduce the human effort of walking. It achieves this by emulating the calves with a mechanical spring [33]. Therefore, supplementing the work done by the body with a passive mechanical component is well founded in both theory and practice.

\section{A.1.2 Anthropometric Data}

The physical dimensions of body segments vary greatly between individuals. Anthropometric data is used to provide representative average data relating to various physical parameters for the general population.

Numerous investigators have measured segment mass properties of living subjects or cadavers. This has been used to develop empirical formulas for estimating body segment parameters for a given subject on the basis on easily obtained information such as height and weight [69]. This concept is illustrated in Figure A.5, which displays some values for segment length that have been summarized by D. A. Winters [70]. These values are only approximations as they represent averages for sample populations of subjects. This information is used as a guide when body dimensions are needed during the design phase. 


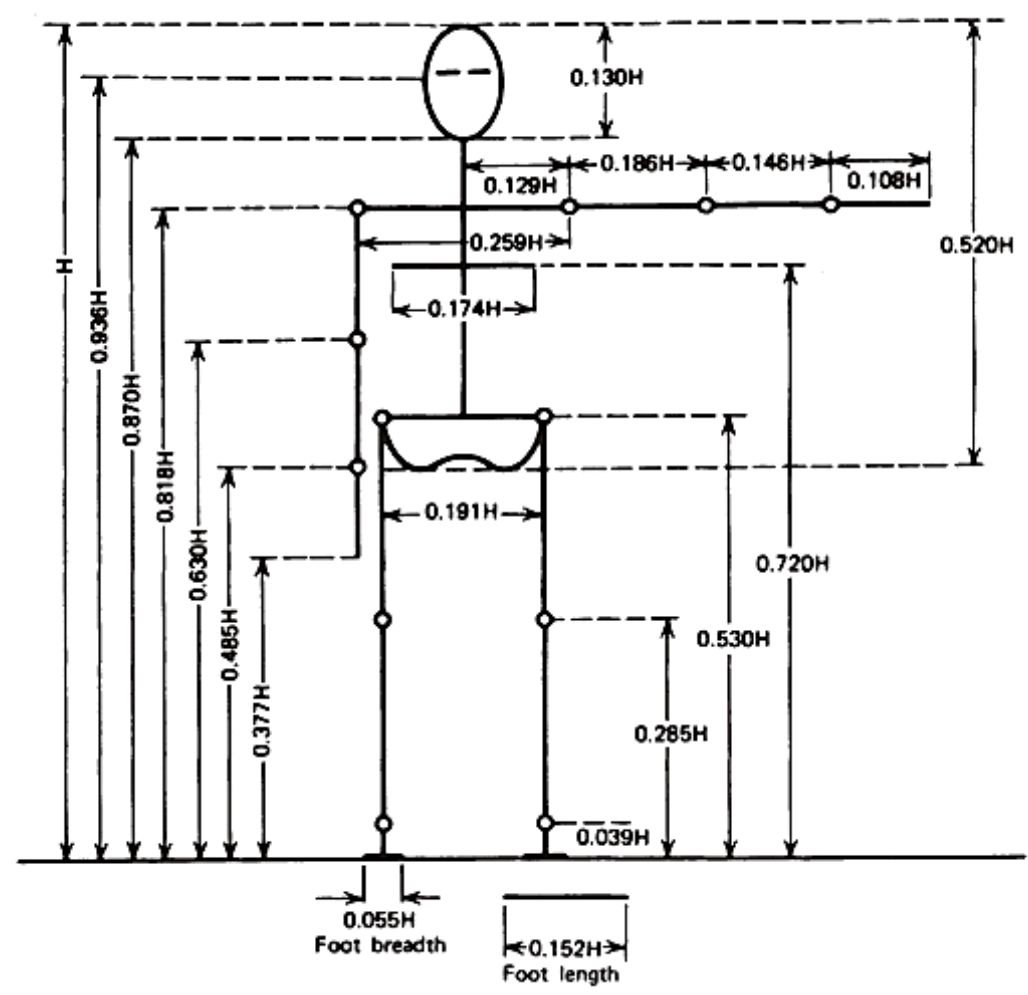

Figure A.5: Various segment lengths expressed has a function of body height [70]

\section{A.1.3 Fundamentals of Gait}

To present the various phases of walking, the gait cycle is often described in terms of strides. One stride is a heel to heel measurement of one complete walking cycle on the same leg, as presented in Figure A.6. A step is a measurement of the distance between the heel of one foot and the heel of the opposite foot. Cadence, or the walking rate, is calculated in steps per minute. The product of the cadence and step length defines the velocity. 


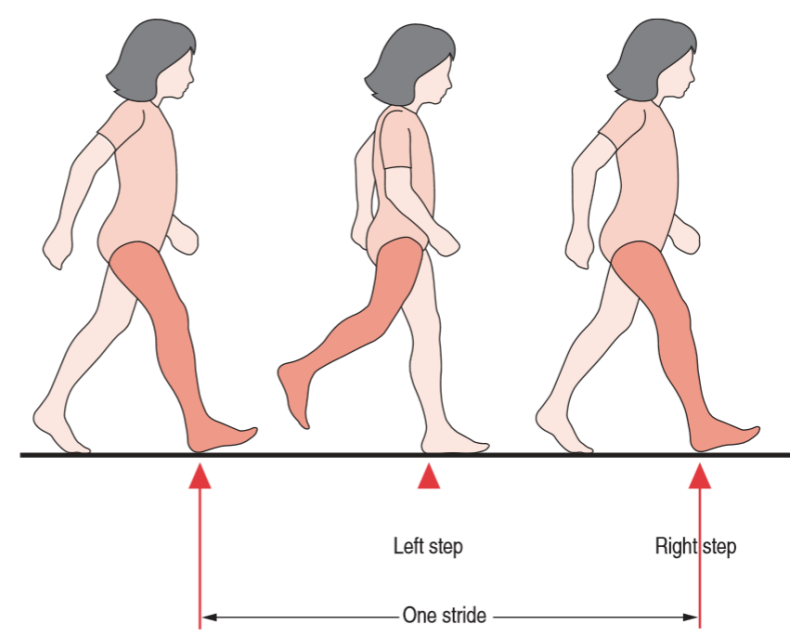

Figure A.6: Illustration of one full stride. Adapted from [71]

\section{The Gait Cycle}

As illustrated in Figure A.7, the natural gait pattern can be divided into two main phases namely the stance and swing phase.

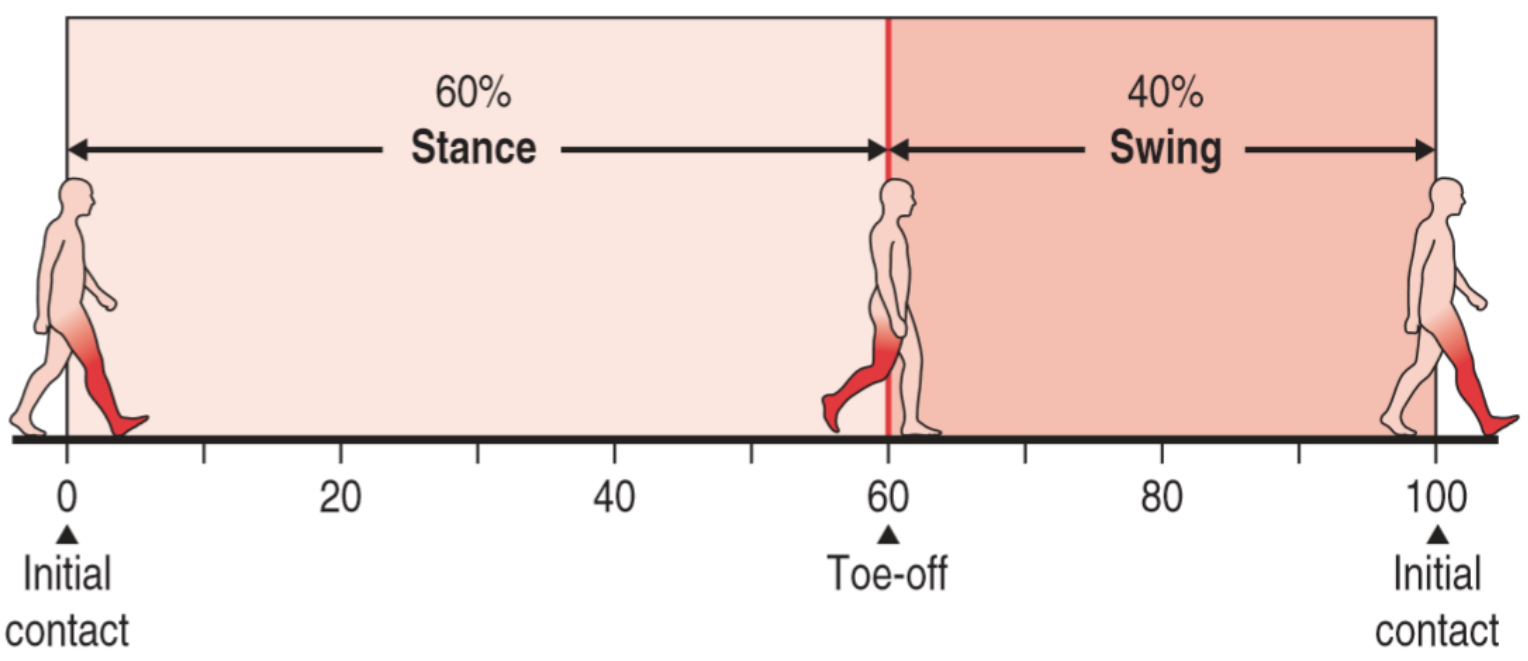

Figure A.7: The Gait cycle showing the division of the stance phase and the swing phase [71]

During walking, the gait cycle is further divided to accurately describe the distinct kinematics of the lower limbs. 


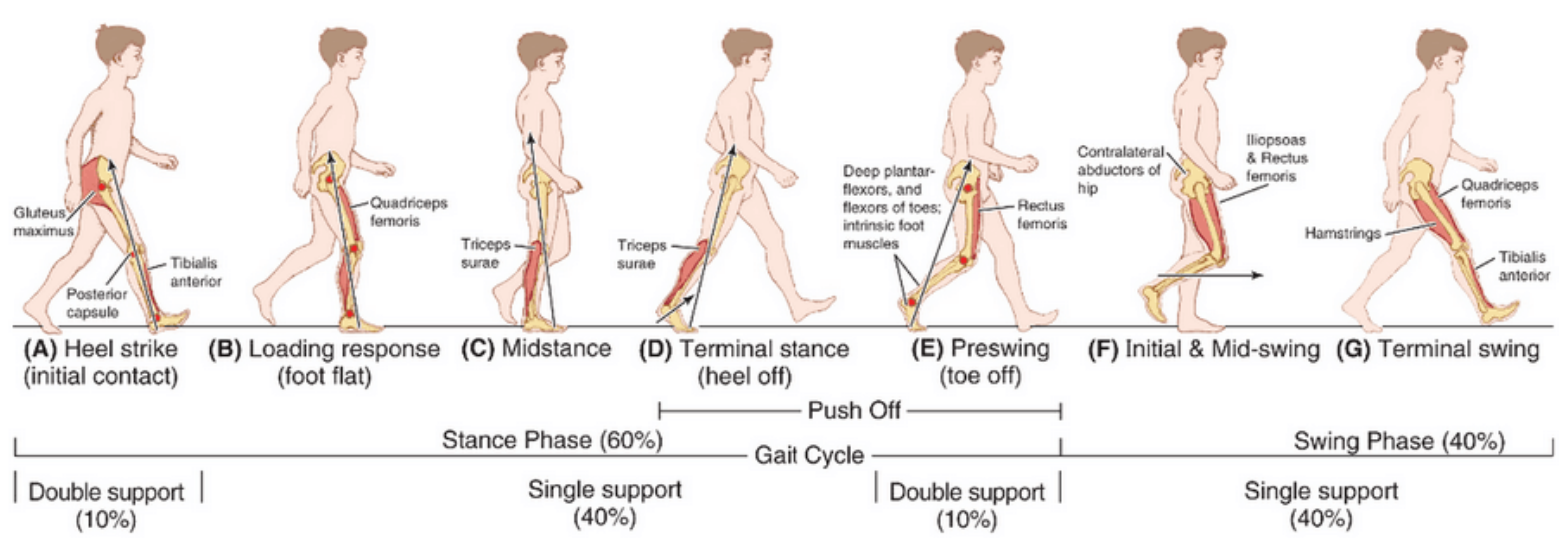

Figure A.8: Gait cycle illustrating different stages and primary muscles active during each phase. The black arrows illustrate the direction of the ground reaction forces during stance phase. During the swing phase these arrows indicate the advancement of the limb [72]

\section{Stance}

Stance is divided into 5 phases: Initial contact (A), loading response (B), mid stance $(C)$, terminal stance $(D)$ and pre-swing $(E)$, shown in Figure A.8.

A Initial contact is simply the instant when the heel touches the ground. Immediately following heel strike weight begins to be transferred to the supporting leg. The Gluteus maximus is active in order to help push the straight limb into the ground and prevent knee buckling. Also active during this stage, is the Tibialis Anterior. As the support limb begins to be loaded this muscle controls the plantar flexion of the foot in order to prevent rapid drop in the toes, also called "foot slap".

B Loading response is characterized by the transfer of the body weight to the supporting leg. While the support leg is being loaded the contralateral leg is preparing for swing. During the acceptance of weight the knee will flex slightly. The Quadriceps muscles contract eccentrically and then concentrically in order to absorb impact forces and to smooth out the trajectory of the center of mass.

C Mid stance is characterized by the progression of the center of mass over the supporting foot. During this phase, the supporting leg is straight. The momentum of the center of mass carries it forward. During this phase very little muscle activation is observed. The triceps surae is gradually stretched during 
this phase.

D Terminal stance corresponds to the heel strike of the contralateral leg. This phase includes the generation of a large plantar flexion ankle torque.

E Pre-swing is the last phase and counts for $10 \%$ of the Gait Cycle. At this point, both feet are in contact with the ground. This phase ends with the toe lifting off the ground and marks the end of the stance portion of gait

\section{Swing}

Initial and mid swing $(F)$, and terminal swing $(G)$, describe the advancement of the trailing limb and how it is prepared for the next step.

F The Initial and mid swing phases are grouped together in Figure A.8. During this portion of the gait, the contralateral hip generates an abduction moment in order to prevent excessive pelvis tilt. The hamstrings are also active to flex the knee to allow the foot to clear the ground. Lastly the hip flexors are active in order to advance the limb.

G Terminal swing is the final stage of the gait cycle. During this phase, the hamstrings activate eccentrically in order to slow the momentum of the shank and to prepare the limb for heel strike. The antagonist positioning of the quadriceps allows for precise knee flexion control in order to ensure that the limb is extended prior to heel strike. The tibialis anterior is also active to dorsiflex the foot.

\section{A.1.4 Ground Reaction Forces}

During the gait cycle the Ground Reaction Forces (GRF) vary continuously. The amplitude of these forces can be illustrated by the so called "butterfly diagram", as presented in Figure A.9. This diagram illustrates that the ground reaction force varies above and below resting body weight during stance. When the limb is loaded the reaction forces rise quickly and overshoot the body weight. The reaction forces then diminish slightly in the mid stance phase before rising again during terminal stance. The velocity of walking has a direct effect on the magnitude of this overshoot. In the 
swing phase, there are no reaction forces on the studied limb as it is not in contact with the ground.

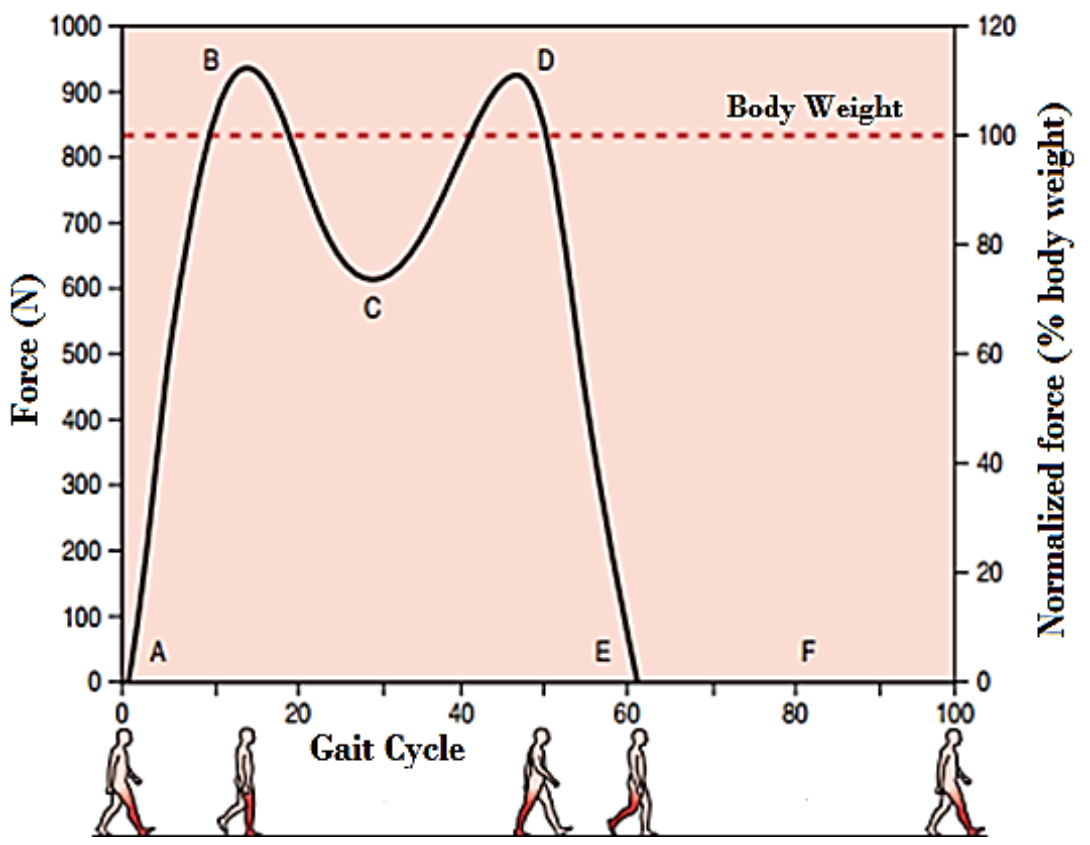

Figure A.9: GRF in function of gait phase of walking modified from [73]

The point of application of force also varies throughout stance. When the foot first makes contact with the ground, the heel is transferring the body weight forces to the ground. As the center of mass of the individual advances, so to, does the point of application on the foot. Near the end of the stance phase, the foot plantar flexes and it is the ball of the foot and the toes that transmit the final forces to the ground. Figure A.10 illustrating the magnitude, direction as well as the point of application of the ground reaction forces during walking gait cycle. The progression of the ground reaction forces is from heel to toe. 


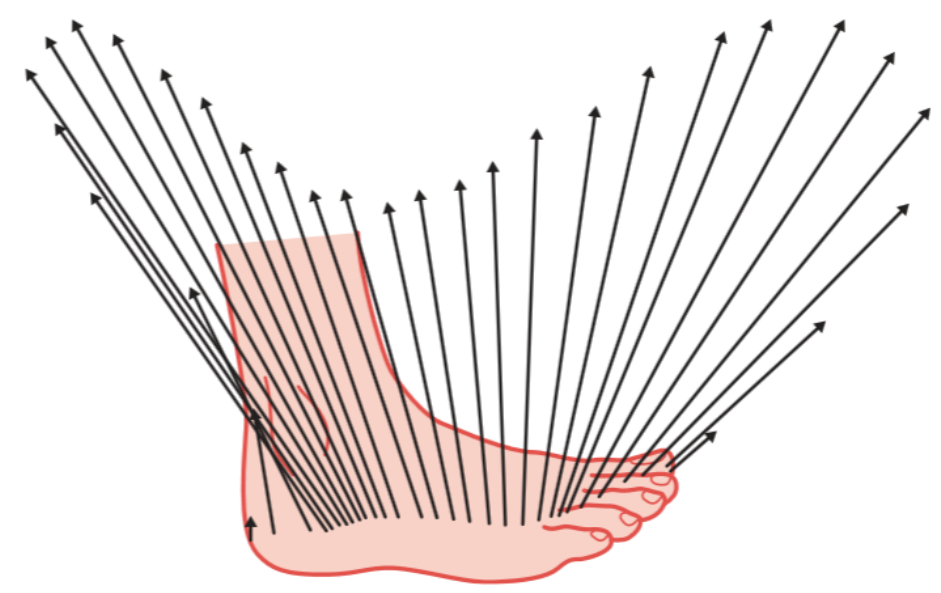

Figure A.10: Butterfly (or Pedotti) diagram modified from [73]

\section{A.1.5 Gait Energy Analysis}

Due to the complexity of the biological system, human movements typically involve multiple structures. Therefore, the origin of force generation and the usage of energy inside the body are challenging to quantify. In order to examine the effectiveness of energy saving devices, there needs to be a way to quantify human exertion. Currently the most widely used method of measuring human effort is the oxygen consumption rate. This method consists of a face mask that monitors the flow and composition of the gases breathed by the individual. Increases in oxygen consumption are directly linked to increases in effort. However, this technique considers the body as a whole and has no way of determining which muscle groups are consuming the most energy. To quantify the effort of individual muscles the current standard is electromyography (EMG) measurement. This technique uses electrodes to measure the electrical activity in a muscle. This is then compared to other muscle groups in order to isolate the biological structures most active during a certain movement.

\section{Measurement of metabolic cost using oxygen consumption rate}

As the body uses chemical energy (in the form of ATP) it is replaced by the breakdown of precursors such as carbohydrates and fats. Once in a steady state exercise of low to moderate intensity, the energy reserves inside the muscles themselves have been depleted and the aerobic production of ATP is responsible for the continued function of the body. This process can only be done in the presence of oxygen. 
Therefore if it is known that the body is working in this aerobic state, it is assumed that $100 \%$ of the ATP energy is being generated by cellular respiration in the presence of oxygen. Consequently, direct monitoring of the consumption of oxygen is used as a measure of effort.

Various types of respirometers exist to measure the gases breathed by living organisms. In the case of human testing, volumetric flow rates are obtained. These values are usually normalized by patient weight and speed of locomotion yielding $\mathrm{VO}_{2}$ as the volume of $\mathrm{O}_{2}$ gas inspired per $\mathrm{kg}$ of mass per meter traveled $\mathrm{mlO}_{2} * \mathrm{~kg}^{-1}$ $* m^{-1}$. The amount of oxygen consumed during a certain activity depends on a number of factors, including size, sex, and level of fitness. Therefore when testing assistive devices, a baseline trial is usually carried out and compared to subsequent tests. In 2008 Sawicki and Ferris [10], illustrate this type of metabolic measurement. The authors used an ankle exoskeleton powered by a PAM and an air compressor, see Figure A.11, in an attempt to reduce the metabolic cost of walking. Surface EMG sensors were used to measure the activation of the calf muscles, and trigger the inflation of the PAM to complement the user.
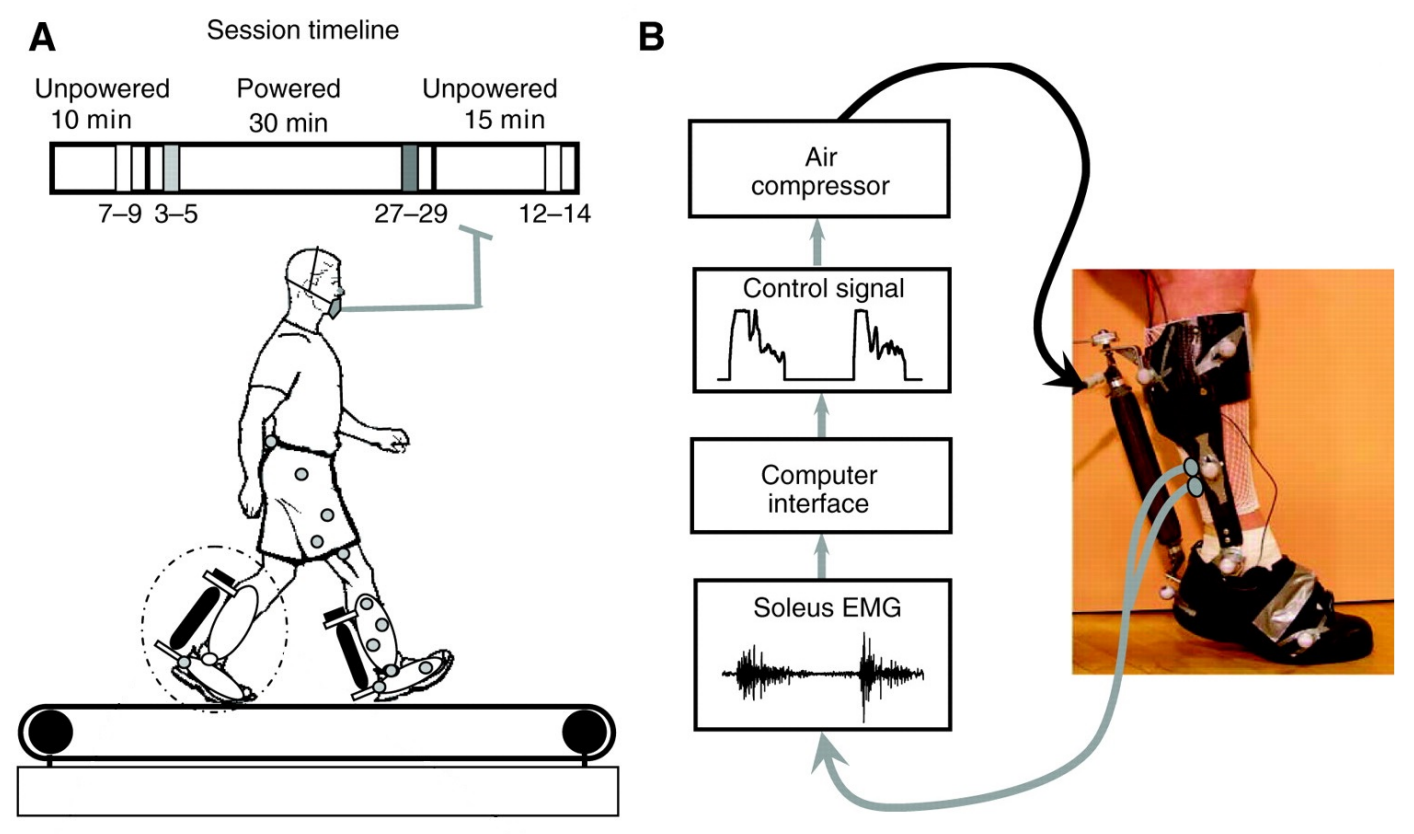

Figure A.11: Experimental set up. A) shows the participant on an instrumented treadmill wearing a respirometer. B) illustrates how the muscle activity of the Soleus was captured with surface electromyography then the pneumatic muscle was activated to compliment the joint movement. Modified from [10] 
The test results indicated that wearing the device increased the metabolic cost of walking by $7 \%$ when compared to the baseline. This was hypothesized to be due to the test subjects' unfamiliarity with the device. As the test continued, the metabolic cost of walking decreased by $10 \%$ when compared to the baseline. The results from this study indicate that supplying a plantar flexion torque during the push off phase can reduce the energetic cost off walking.

\section{Measurement of muscle activation using electromyography}

When the brain wants to contract a muscle, a voltage is sent along a motor neuron to the muscle belly. Electromyography is a technique that allows for the measurement of these voltages. Furthermore, the intensity of the muscle contraction is directly related to the magnitude of the measured voltage. Therefore, the increase in recorded voltage corresponds to an increase in muscle activation and thus in metabolic cost. The use of EMG is well established in research. For example, in a 2009 study by Kao et al. [74], the authors measured the muscle activation during walking while subjects wore an actuated ankle exoskeleton. The use of an actuator positioned in parallel with the shank was intended to directly replicate the function of the Triceps Surae. The study results showed a 30\% decrease in the activation of the Soleus muscle. From this result it appears that the use of an assistive device can partially replace the role of the muscles.

In certain situations, it is valuable to use both EMG and oxygen uptake methods to quantify human effort. A study by Browning et al. [75], examined the effect of adding mass to the lower limbs on the energetics of walking. The researchers placed weights on the feet, shanks, thighs as well as the waist. The muscle activation was recorded using electromyography and the overall human effort was measured via oxygen uptake rate. Figure A.12 illustrates the EMG data obtained for various loading

scenarios. The results show that not only does the increasing foot mass increases the activation of the muscles in the calf, it also increases the activation of muscles higher up the kinematic chain. The authors hypothesized that adding mass to the foot has a large impact because it is farthest from the point of rotation of the leg (i.e. pelvis). 

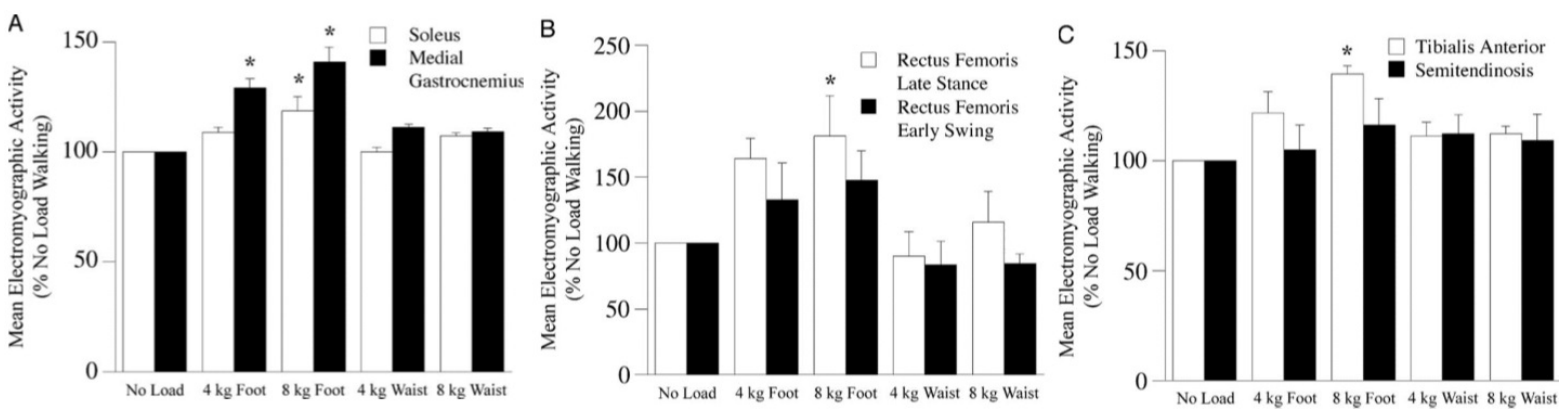

Figure A.12: Normalized mean electromyogram vs load condition relative to unloaded walking for the soleus and medial gastrocnemius during midstance (32-52\% stride) (A), the rectus femoris during late stance (57-62\% stride) and early swing $(62-67 \%$ stride) (B), and the tibialis anterior and semitendinosus during late swing (90-100\% stride) (C). Values are means for all subjects. EMG magnitudes tended to increase with foot loading in the soleus, medial gastrocnemius, and rectus femoris during stance and the tibialis anterior during swing. Image and caption taken from [75]

The energetic consumption of the individual was also examined and can be seen in Figure A.13. This graph demonstrates that added mass has the highest impact when it is placed on the foot. It also demonstrates that with a mass of only $4 \mathrm{~kg}$, the net metabolic rate increased significantly. Furthermore, with an added foot mass of 8 $\mathrm{kg}$ the net metabolic rate rose by $48 \%$. 


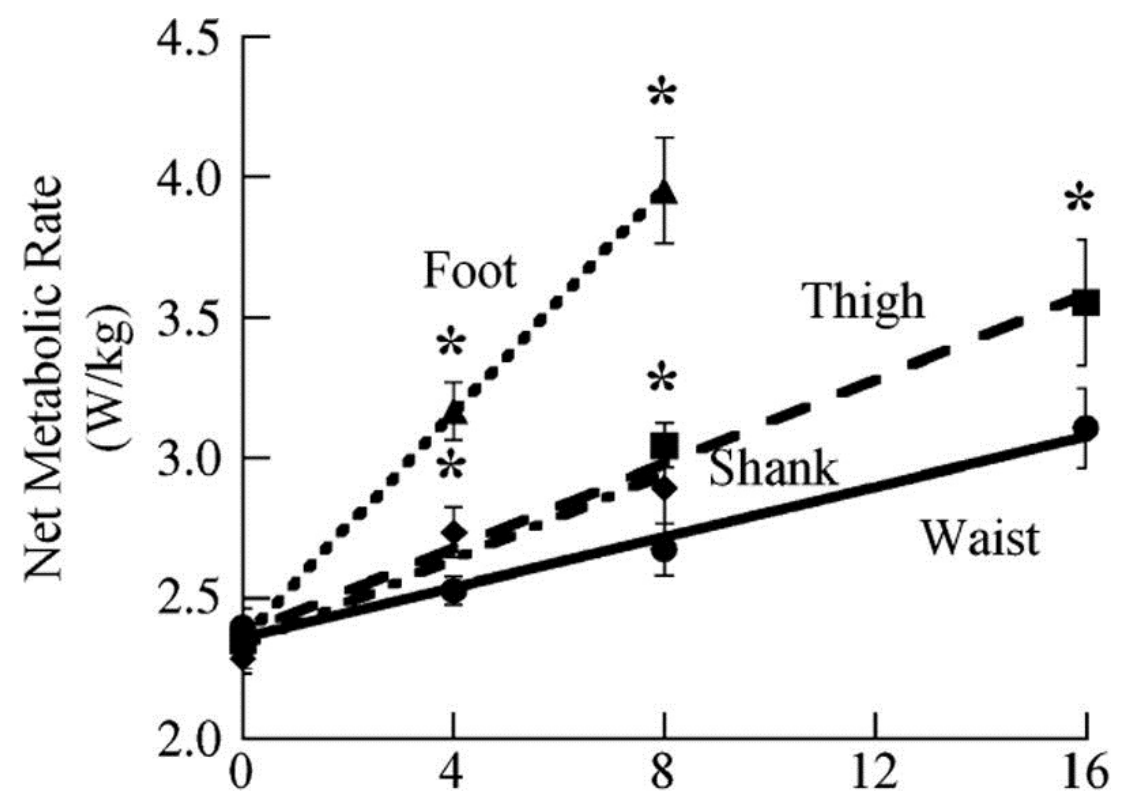

Figure A.13: Mean net metabolic rate $(\mathrm{W} \cdot \mathrm{kg}-1)$ vs load mass $(\mathrm{m})$ and location. The increase in net metabolic rate was greatest with foot loads, similar for thigh and shank loads, and least for waist loads. *Significant difference, load location vs waist load. Image and caption taken from [75]

This study illustrates the effect that added foot mass can have on the energy expenditure of walking. It also shows that as the mass of the foot increases, the muscular activation of the calf must increase to compensate. In this case the oxygen uptake method measured the overall human effort whereas the EMG was used to specifically target certain muscles of the lower limbs.

The exoskeleton presented in this thesis and described in Chapter 4, is attempting to decrease the effort of a specific muscle group, for this reason, EMG is used.

\section{Gait Analysis and Inverse Dynamics}

The most common gait analysis method is motion capture. The basic function of the system is relatively simple, a participant wears reflective markers at strategic positions on their body. An array of infra-red cameras records the movements of the markers. The marker positions are fed into a software system that generates a $3 \mathrm{D}$ reconstruction of the trajectories of these markers in space. Since the markers were placed at specific anatomical landmarks, a computer generated skeleton can be merged with the points. Once all of this processing has been completed, the kinematics of the body can be computed. The use of motion capture is often merged with 
a complementary data acquisition system that records the ground reaction forces. While the participants are being filmed by the motion capture system, force plates under the foot are recording ground reaction force vectors. With these two sources of data, the inverse dynamics method can be applied.

Inverse dynamics is an analytical approach frequently used to study human gait [76]. It breaks the body into rigid segments connected by joints. It subsequently uses the kinematics from the motion capture system and the ground reaction forces from the force plates to estimate the joint reaction forces. The method is useful because it generates numerical values that describe human motion in a mechanical way. This allows engineers and researchers to quantitatively evaluate forces inside the body during human movement. The angular velocities and accelerations about various joints combined with the known external forces enables the calculation of joint moment, work, and power. Figure A.14 shows plots of joint angles, joint moments, joint power, as well as the main flexor and extensor muscles responsible. 

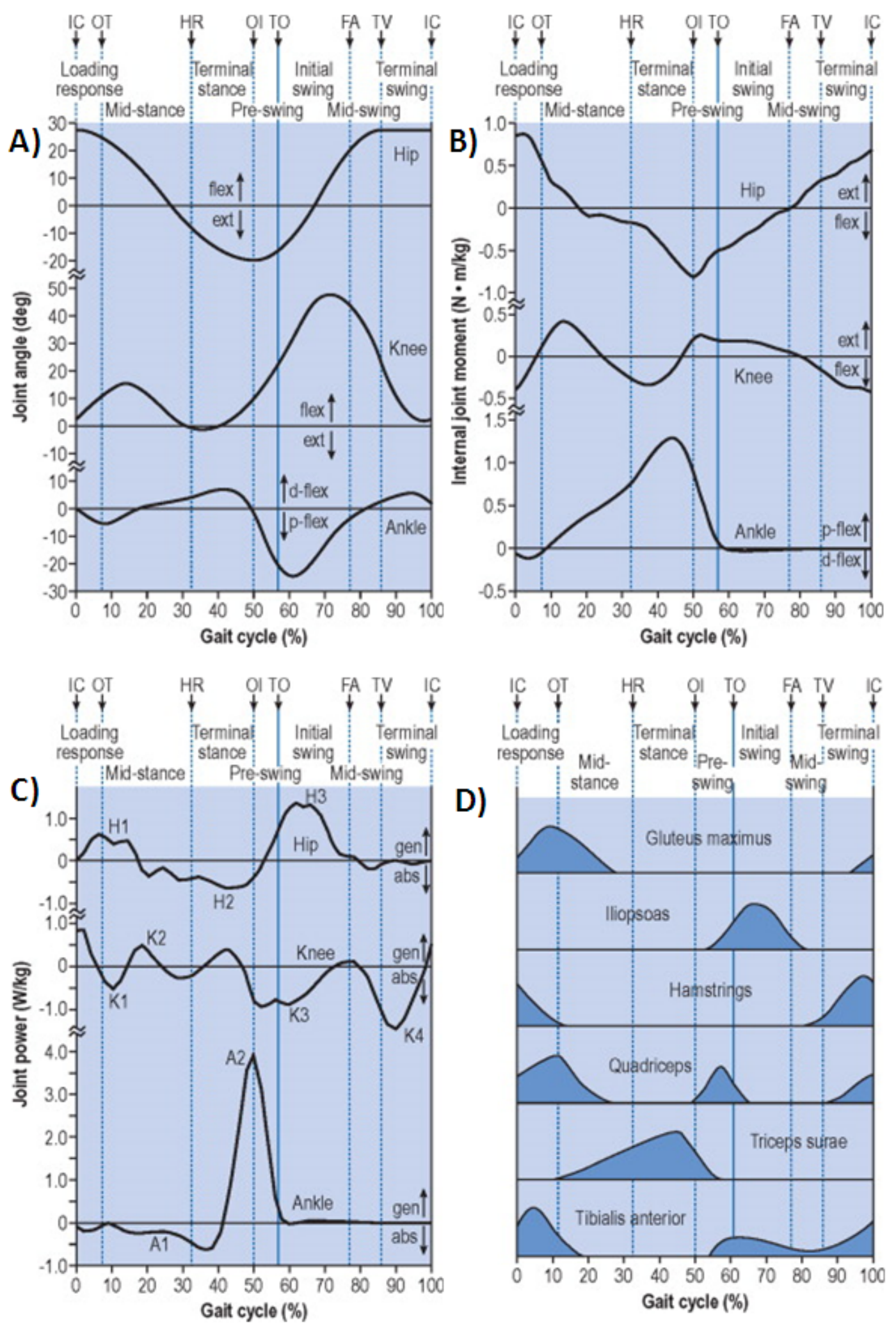

Figure A.14: Graphical illustration of gait data for the hip knee and ankle joints during healthy walking. Values are normalized by body weight A) Joint angles [Degrees] B) Joint Moments [ $\mathrm{N}^{*} \mathrm{~m} / \mathrm{Kg}$ ] C) Joint Power [W/Kg] D) Illustration of active muscles during gait cycle. Modified from [6] 
From healthy gait data such as that presented in Figure A.14, certain patterns are visible. For instance, graph B) demonstrates the large moment present at the ankle during gait. From the beginning of the stance phase (at $0 \%$ ) there is a gradual increase in the moment at the ankle followed by a sudden drop just before the foot leaves the ground and enters the swing phase. Figure A.14 C) illustrates the large power input during this time. Part D) shows that the Triceps Surae is responsible for this moment generation. Therefore, the Triceps Surae is responsible for a large portion of the energy required to walk.

Figure A.14 also graphically illustrates the angle moment and power for the hip knee and ankle joints. It can be seen that in Figure A.14 C) the power fluctuates above and below the zero axis. The positive portions correspond to a generation of power and the negative portions correspond to an absorption of power. This is more clearly shown in Figure A.15 which depicts the power at the knee and ankle joints during one complete stride of a healthy human, normalized by body weight.
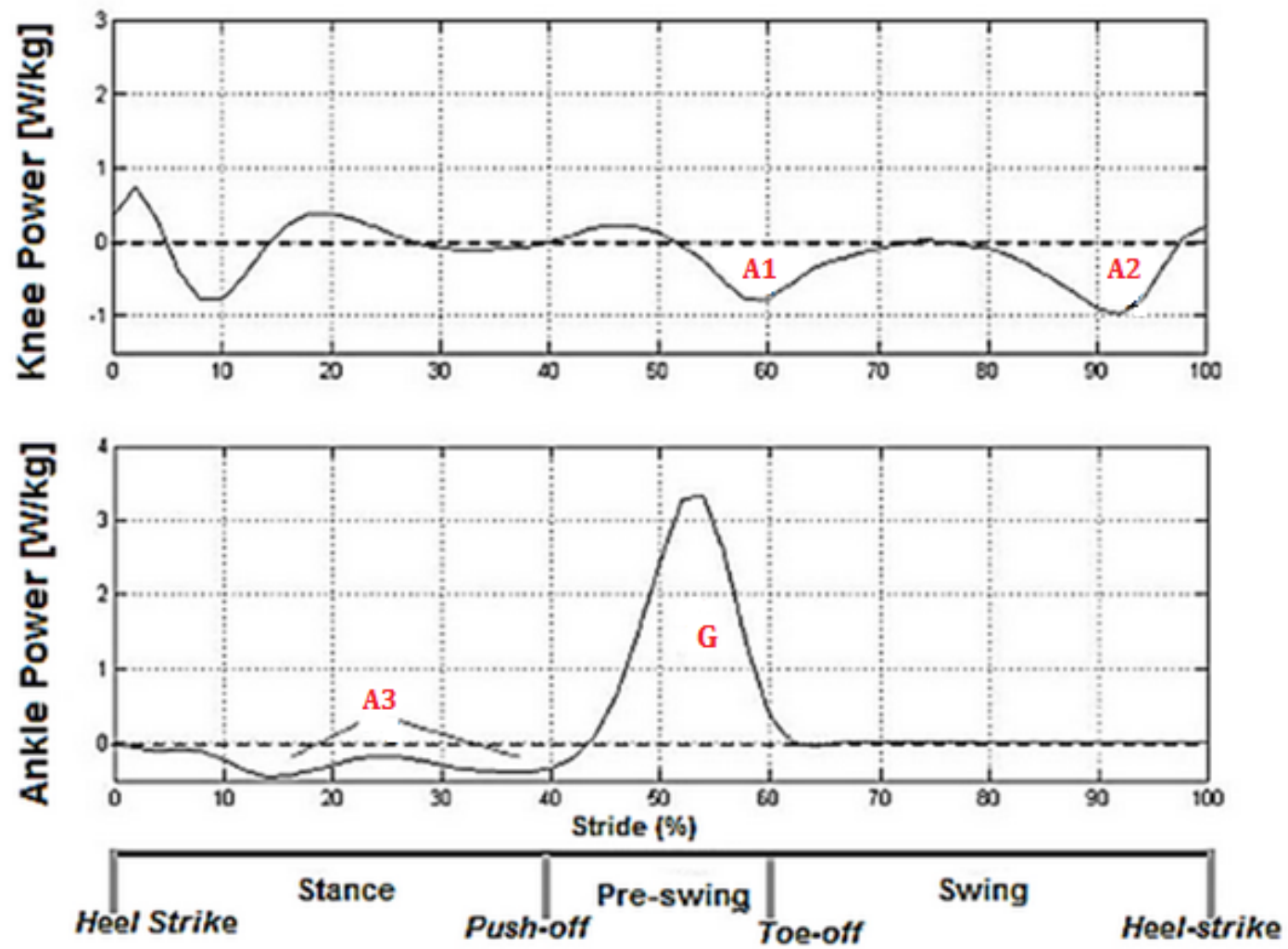

Figure A.15: The joint power of the ankle and knee of healthy human gait over one full stride, normalized by body weight. Modified from [7]

Figure A.15 highlights three notable instances, namely, heel strike, push-off and 
toe-off. These instances divide the stride into three distinct section. Namely the stance phase, pre-swing phase and the swing phase. The areas A1, A2, A3 indicate the energy absorption, whereas $\mathrm{G}$ indicates the energy generation.

- Stance: the knee absorbs a small amount of energy during loading response and works to keep the limb extended. In the meantime, the ankle joint absorbs energy (represented by A3 in FigureA.15).

- Pre-swing: the knee passively absorbs energy, represented by A1, while the ankle generates the main part of the energy for the push-off, represented by G, which is about the $80 \%$ of the overall power generation during gait.

- Swing: during the late swing phase the knee absorbs energy to decelerate the limb, this is represented by A2 in the figure. Meanwhile, the energy generation in the ankle joint is negligible.

Figure A.15 illustrates that in the healthy human gait, the knee joint is mainly an energy absorber. However, the ankle joint demonstrates nearly a complete balance between the generated and the absorbed energy, since the energy for push-off generation $(G)$, is nearly equal to the total energy absorbed in A3. 


\section{Appendix B}

\section{Instrumentation and measurements of PAM test- ing}

\section{Pressure sensor system}

The pressure inside the PAM is a key variable that directly affects the behaviour of the muscle. In order to monitor the pressure, a $61 \mathrm{CP}$ Series ceramic capacitive pressure transducer from Texas Instruments was used. The transducer can be seen in Figure B.1 A.
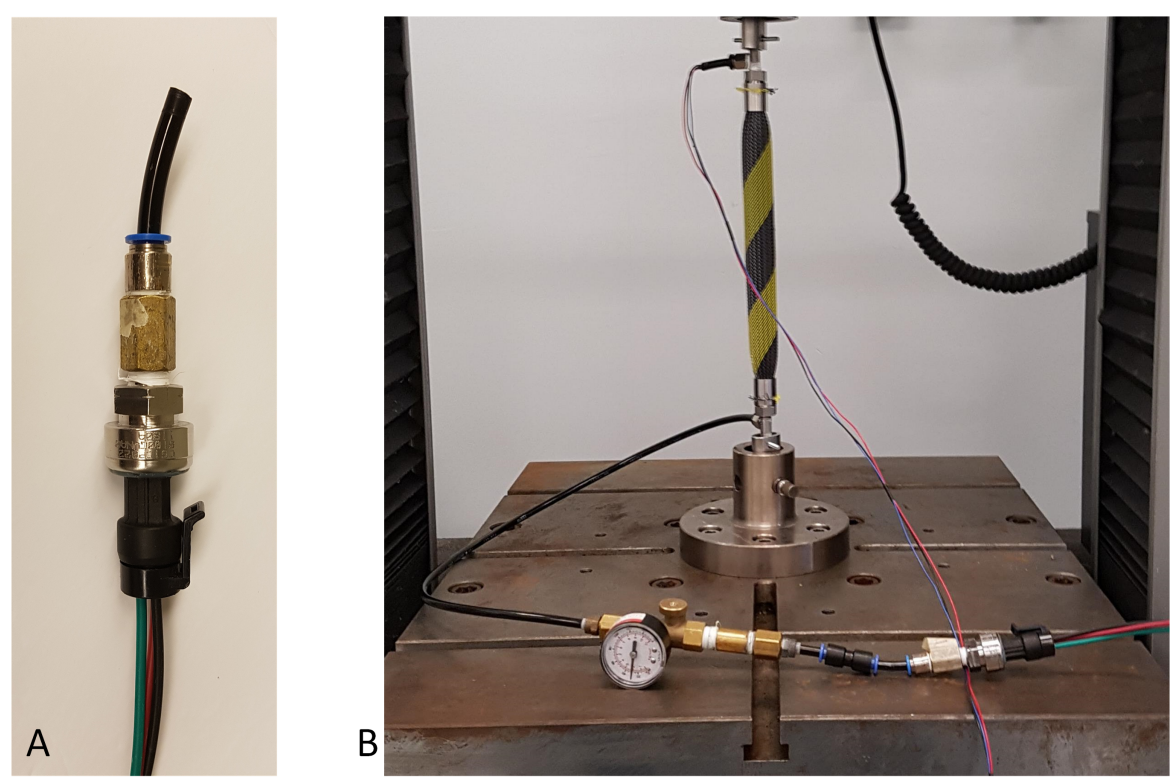

Figure B.1: A) shows the pressure transducer, B) shows the hose and valve connecting the transducer to the PAM 
The transducer is connected to the PAM via a short hose with a valve at the end. Once inflated the valve is closed and the air supply is disconnected and replaced with the pressure transducer. The valve is then opened to allow the gas to reach the transducer. This valve is shown in Figure B.1 B. The hose connecting the PAM to the pressure transducer holds a volume of gas equal to approximately $5 \%$ of the volume of the PAM.

The transducer has three wires connecting it to the micro controller. The voltage is read by the Arduino Mega 2560 micro controller. The voltage is imported into the computer using CoolTerm software. The system was calibrated by applying a known input pressure from 0-80 psi using 10 psi intervals. The voltage was recorded at each step and used to generate a linear relation between the applied pressure and measured voltage. This calibration was performed several times during the initial stages of testing before the methodology was finalized.

\section{Temperature sensor system}

The PAM model proposed in this work uses a polytropic pressure model. In most PAM models, the gas is assumed to be isothermal [11],[48],[49]. To measure the true gas temperature inside a PAM, a temperature sensing system was developed. The system uses a TMP 36 temperature sensor made by Analog Devices Inc, see Figure B.2. The sensor is placed inside the gas volume of the muscle during muscle fabrication. The TMP temperature sensor comes pre-calibrated. It was however tested to verify that it functions as intended before being integrated into the PAM. It was inserted inside the muscle and was connected to the Arduino Mega 2560 micro controller for data acquisition.

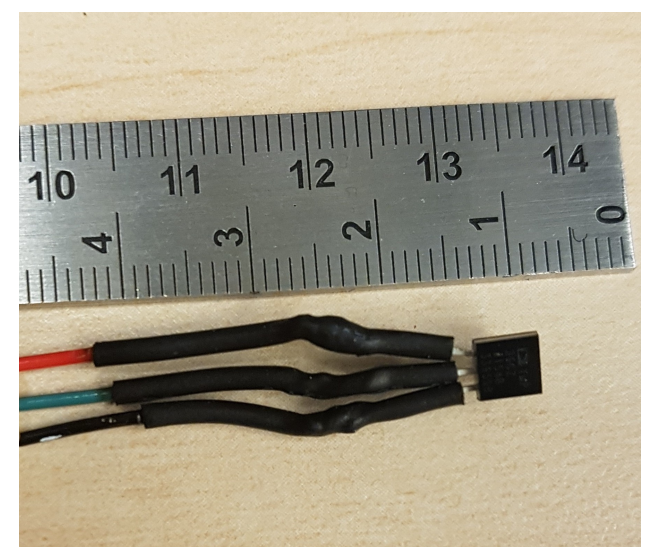

Figure B.2: TMP 36 temperature sensor. The ruler is in $\mathrm{cm}$ 
With the PAM being subjected to high pressures, inserting a sensor creates a risk of leaks. Initially a small wireless bluetooth transmitter was considered. This device would simply be placed inside the muscle during fabrication and would measure the internal temperature. However, a limited battery life and size constraints were problematic. Therefore, wires were physically run into the muscle. To minimize leakage, and to ensure that the muscle still operated as intended, two input end caps were used. Typically, PAM have one end cap that is completely sealed, and one end cap with an orifice used for inflation. Figure B.3 shows the end caps that are normally used (with only one orifice for inflation). The test muscle used two inflation end caps, therefore, both end caps had an orifice for inflation.

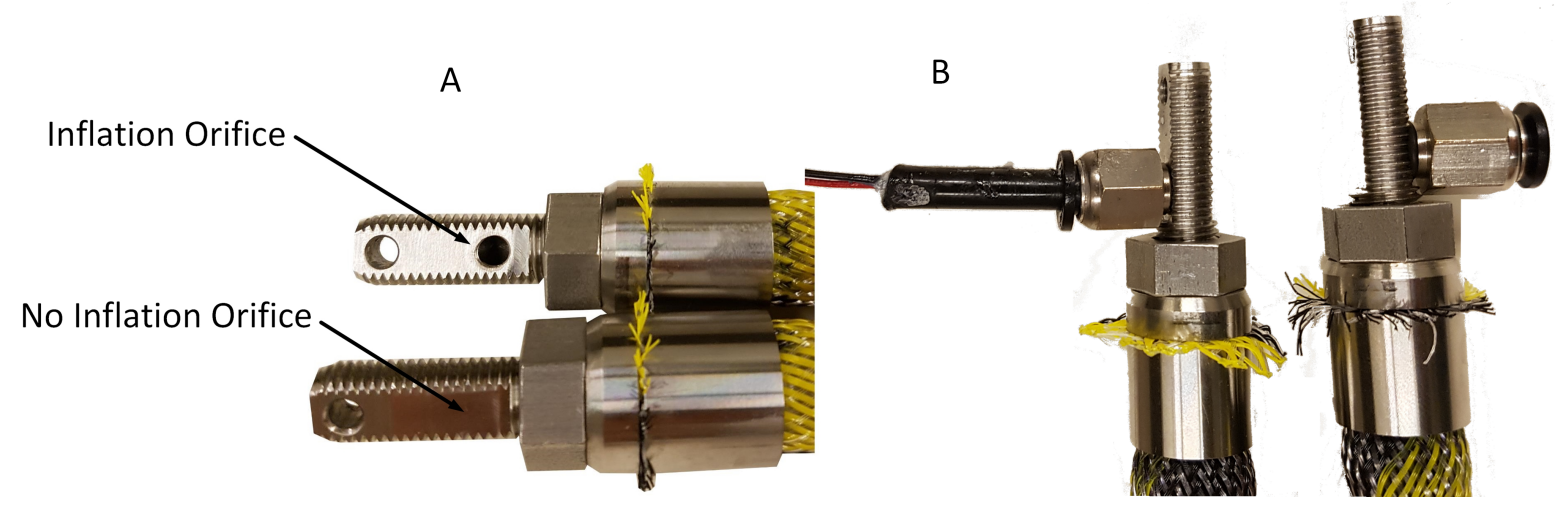

Figure B.3: PAM end caps, A) Normal configuration, one end cap with inflation orifice, the other without. B) Photograph of the PAM end caps showing that both have an orifice for inflation

The wires protruding from one of the end caps extend down into the internal volume of the muscle. The section of tube containing the wires was filled with a polyurethane super glue (Gorilla Glue (C) to seal the tube and prevented air from escaping through this end cap. The other end of the muscle was left un-altered to allow for inflation. 


\section{Appendix C}

\section{PAM specifications and simulation comparison results}

\section{C.1 PAM specifications}

Table C.1: Muscle characteristics for PAM model validation tests for a n2 PAM inflated to $138 \mathrm{kPa}$ and tested at $80 \% 90 \%$ and $95 \%$ of its maximum elongation

\begin{tabular}{lcccc}
\hline Variable Name & Variable symbol & $80 \%$ & $90 \%$ & $95 \%$ \\
\hline \hline Initial muscle diameter & $D_{\text {initial }}$ & $0.030 \mathrm{~m}$ & $0.030 \mathrm{~m}$ & $0.030 \mathrm{~m}$ \\
\hline Initial length & $L_{o}$ & $0.171 \mathrm{~m}$ & $0.171 \mathrm{~m}$ & $0.171 \mathrm{~m}$ \\
\hline Maximum elongation & $e l_{\max }$ & $43.4 \mathrm{~mm}$ & $48.4 \mathrm{~mm}$ & $51.4 \mathrm{~mm}$ \\
\hline Polytropic index & $n g$ & $1-1.4$ & $1-1.4$ & $1-1.4$ \\
\hline Inflation Pressure & $P_{\text {inflation }}$ & $154.65 \mathrm{kPa}$ & $132.79 \mathrm{kPa}$ & $144.31 \mathrm{kPa}$ \\
\hline
\end{tabular}


Table C.2: Muscle characteristics for PAM model validation tests for a n2 PAM inflated to $207 \mathrm{kPa}$ and tested at $80 \% 90 \%$ and $95 \%$ of its maximum elongation

\begin{tabular}{lcccc}
\hline Variable Name & Variable symbol & $80 \%$ & $90 \%$ & $95 \%$ \\
\hline \hline Initial muscle diameter & $D_{\text {initial }}$ & $0.0305 \mathrm{~m}$ & $0.0305 \mathrm{~m}$ & $0.0305 \mathrm{~m}$ \\
\hline Initial length & $L_{o}$ & $0.167 \mathrm{~m}$ & $0.167 \mathrm{~m}$ & $0.167 \mathrm{~m}$ \\
\hline Maximum elongation & $e l_{\max }$ & $46.3 \mathrm{~mm}$ & $52.3 \mathrm{~mm}$ & $55.35 \mathrm{~mm}$ \\
\hline Polytropic index & $n g$ & $1-1.4$ & $1-1.4$ & $1-1.4$ \\
\hline Inflation Pressure & $P_{\text {inflation }}$ & $218.98 \mathrm{kPa}$ & $236.63 \mathrm{kPa}$ & $245.45 \mathrm{kPa}$ \\
\hline
\end{tabular}

Table C.3: Muscle characteristics for PAM model validation tests for a n2 PAM inflated to $276 \mathrm{kPa}$ and tested at $80 \% 90 \%$ and $95 \%$ of its maximum elongation

\begin{tabular}{lcccc}
\hline Variable Name & Variable symbol & $80 \%$ & $90 \%$ & $95 \%$ \\
\hline \hline Initial muscle diameter & $D_{\text {initial }}$ & $0.0314 \mathrm{~m}$ & $0.0314 \mathrm{~m}$ & $0.0314 \mathrm{~m}$ \\
\hline Initial length & $L_{o}$ & $0.165 \mathrm{~m}$ & $0.165 \mathrm{~m}$ & $0.165 \mathrm{~m}$ \\
\hline Maximum elongation & $e l_{\max }$ & $48.35 \mathrm{~mm}$ & $54.3 \mathrm{~mm}$ & $57.3 \mathrm{~mm}$ \\
\hline Polytropic index & $n g$ & $1-1.4$ & $1-1.4$ & $1-1.4$ \\
\hline Inflation Pressure & $P_{\text {inflation }}$ & $305.44 \mathrm{kPa}$ & $316.06 \mathrm{kPa}$ & $298.40 \mathrm{kPa}$ \\
\hline
\end{tabular}


Table C.4: Table of various PAM variables and their values

\begin{tabular}{|c|c|c|c|}
\hline Variable Name & Symbol & Units & Value \\
\hline Diameter of end of PAM & Dend & $\mathrm{m}$ & 0.013 \\
\hline Diameter of deflated PAM & Dref & $\mathrm{m}$ & 0.020 \\
\hline Diameter of deflated bladder & $D b o$ & $\mathrm{~mm}$ & 15.45 \\
\hline Length of hypotenuse of end cone & $L z$ & $\mathrm{~m}$ & 0.0095 \\
\hline Number of fiber revolutions & $n$ & $\mathrm{~N} / \mathrm{A}$ & 2 \\
\hline Number of fibers & $N$ & $\mathrm{~N} / \mathrm{A}$ & 216 \\
\hline Atmospheric Pressure & Patm & $\mathrm{kPa}$ & 101 \\
\hline Specific Gas Constant (dry air) & $R$ & $J / K g K$ & 287 \\
\hline Thickness of bladder & $t b$ & $\mathrm{~mm}$ & 0.254 \\
\hline Thickness of fiber & $t f$ & $\mathrm{~mm}$ & 0.254 \\
\hline Maximum value of angle theta & $\theta_{\max }$ & Rad & 288 \\
\hline Ambient temperature & Tatm & K & 296 \\
\hline \multicolumn{4}{|l|}{ Coefficient of friction between } \\
\hline bladder and fibers & $U b$ & $\mathrm{~N} / \mathrm{A}$ & 0.2 \\
\hline Coefficient of friction between fibers & $U f d$ & $\mathrm{~N} / \mathrm{A}$ & 0.2 \\
\hline
\end{tabular}




\section{C.2 PAM validation results}

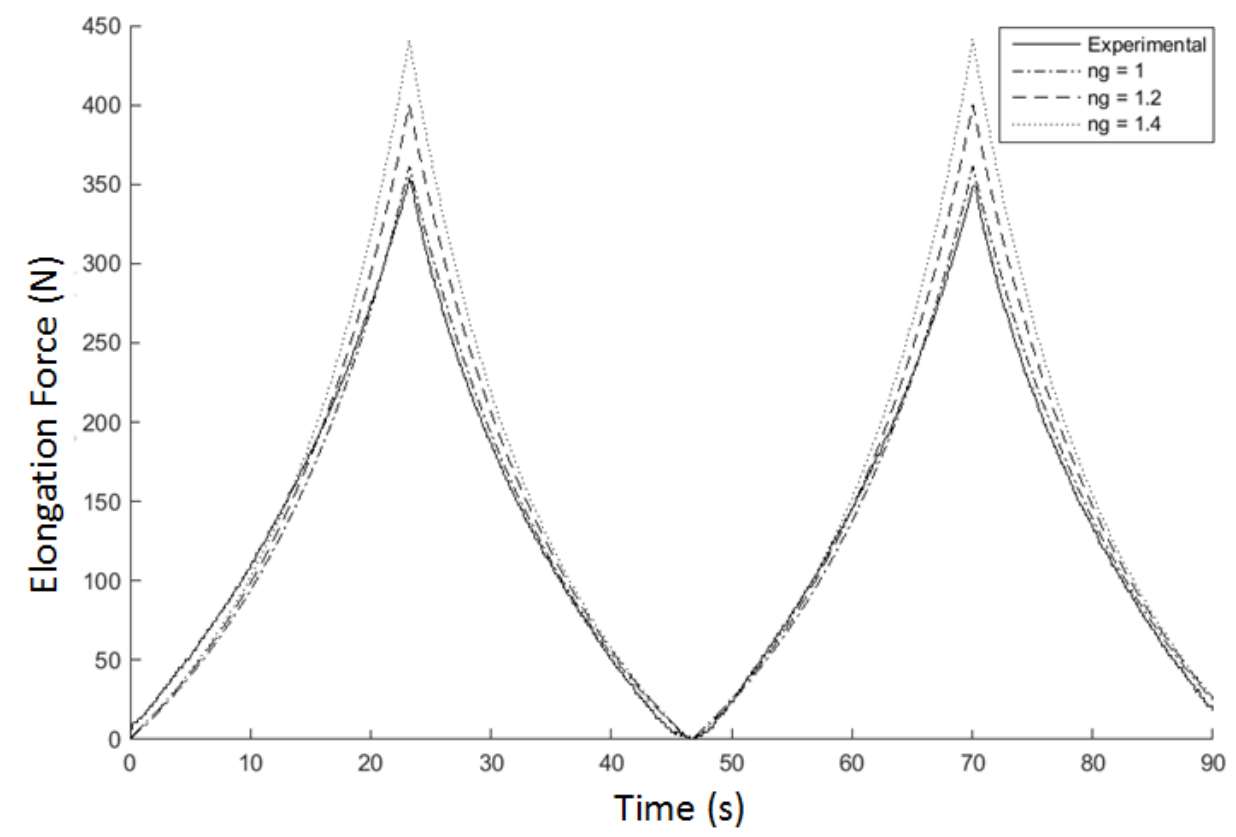

Figure C.1: Force comparison of experimental and simulated results for PAM inflated to $207 \mathrm{kPa}$, tested at $80 \%$ elongation. Bladder stress is not included in this simulation

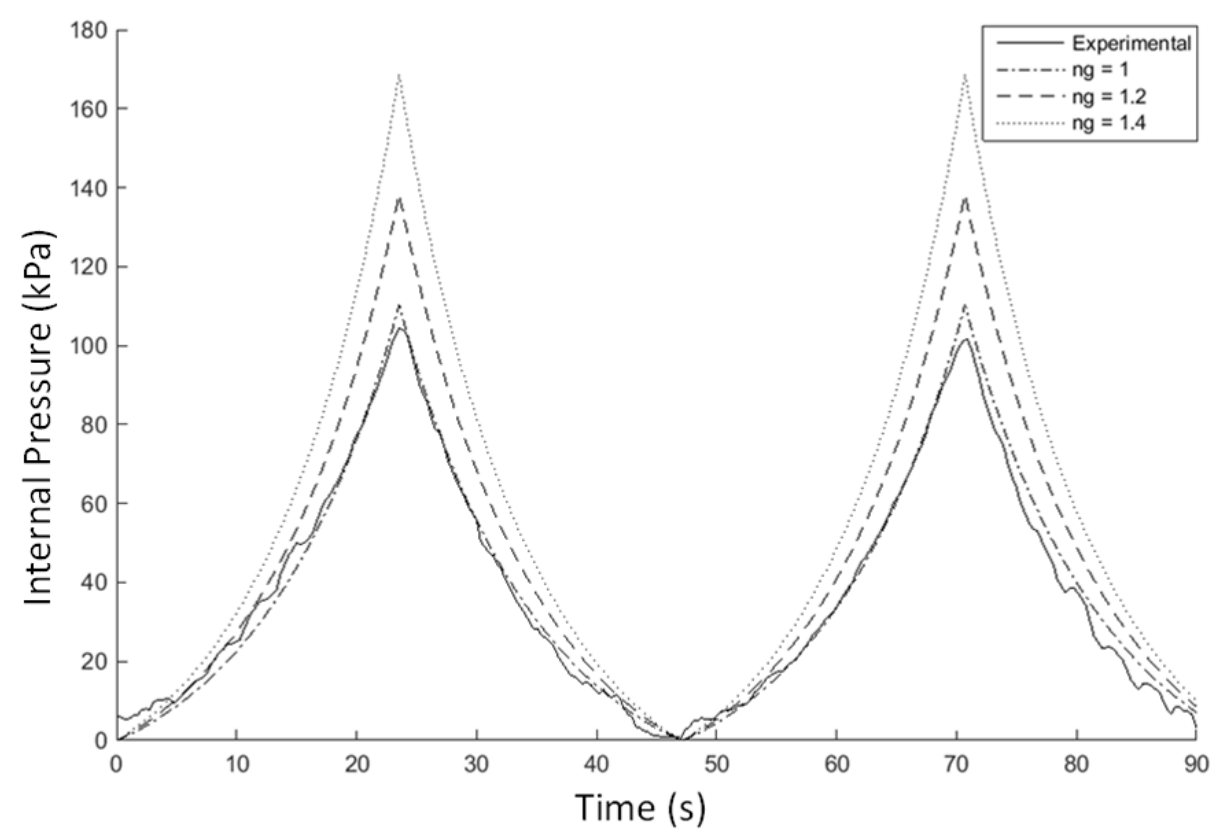

Figure C.2: Pressure comparison of experimental and simulated results for PAM inflated to $207 \mathrm{kPa}$, tested at $80 \%$ elongation. Bladder stress is not included in this simulation 


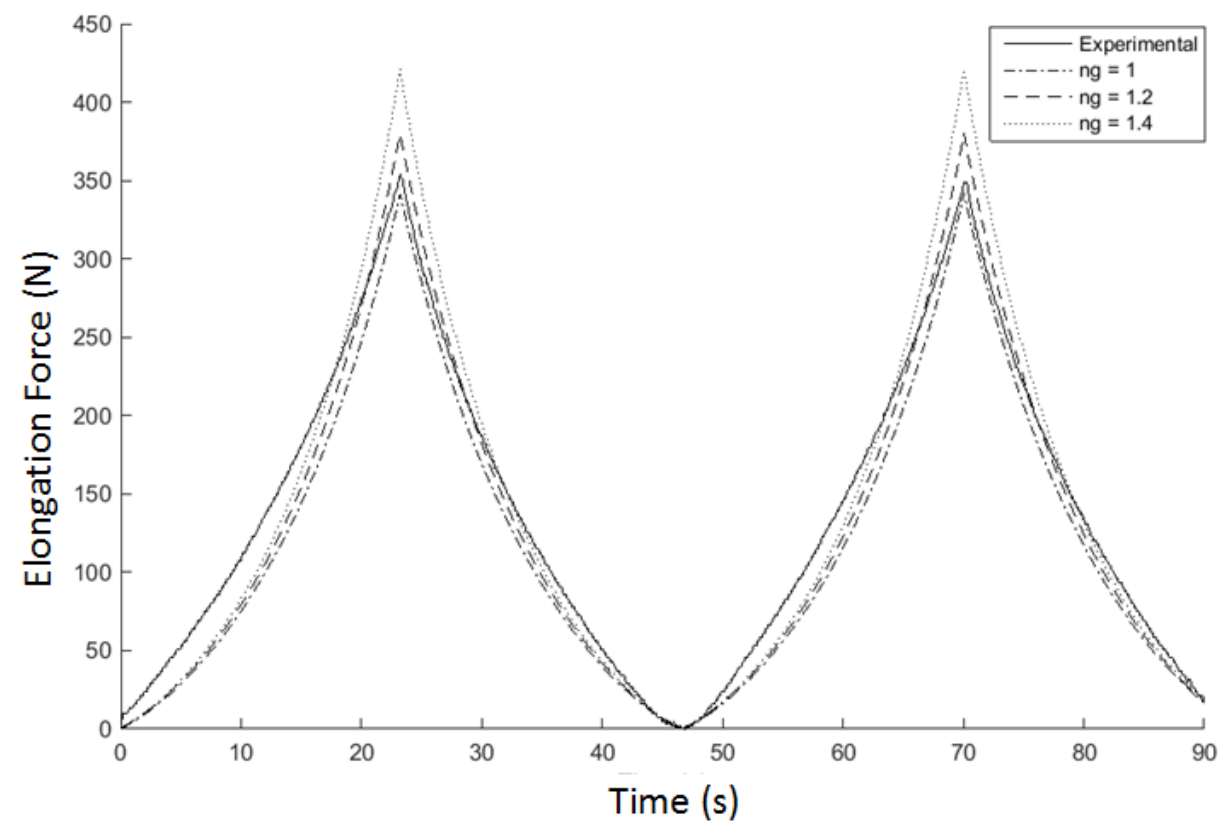

Figure C.3: Force comparison of experimental and simulated results for PAM inflated to $207 \mathrm{kPa}$, tested at $80 \%$ elongation. Bladder stress is indeed included in this simulation

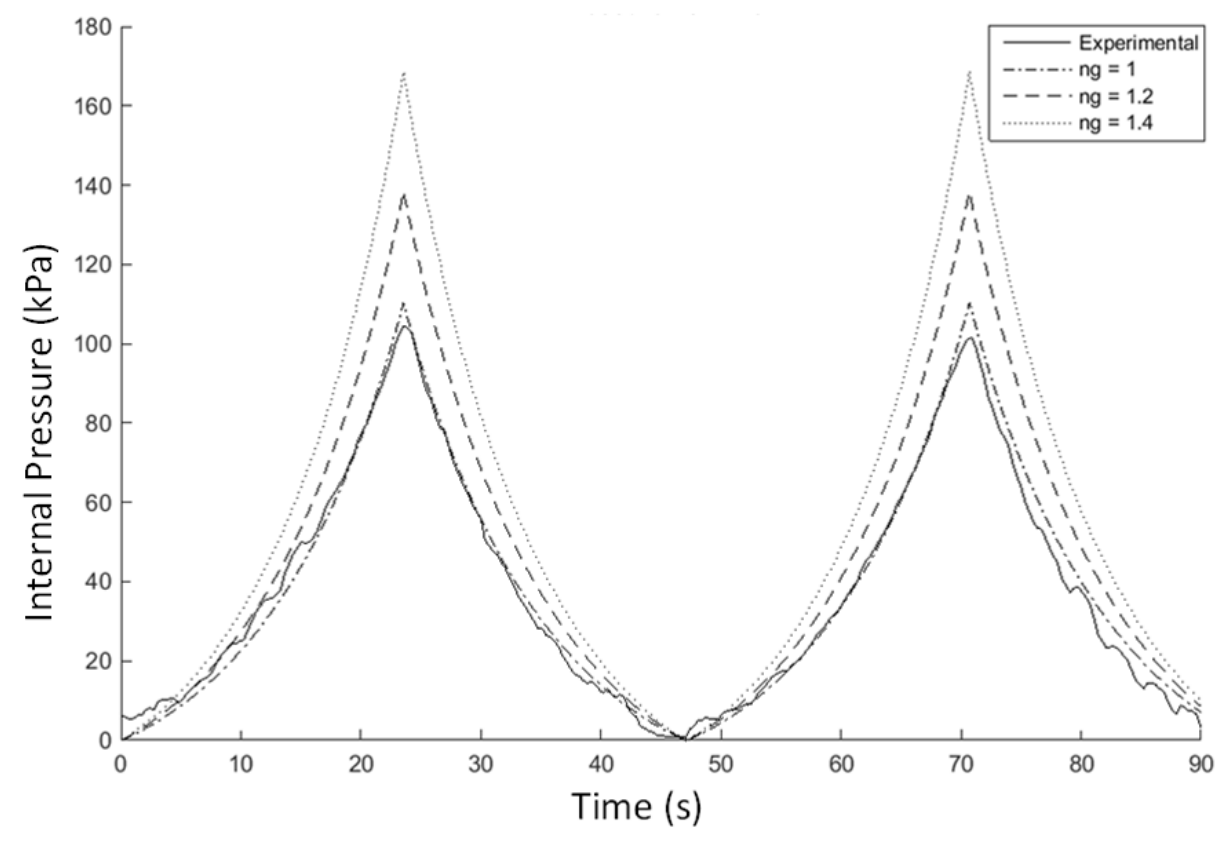

Figure C.4: Pressure comparison of experimental and simulated results for PAM inflated to $207 \mathrm{kPa}$, tested at $80 \%$ elongation. Bladder stress is indeed included in this simulation 


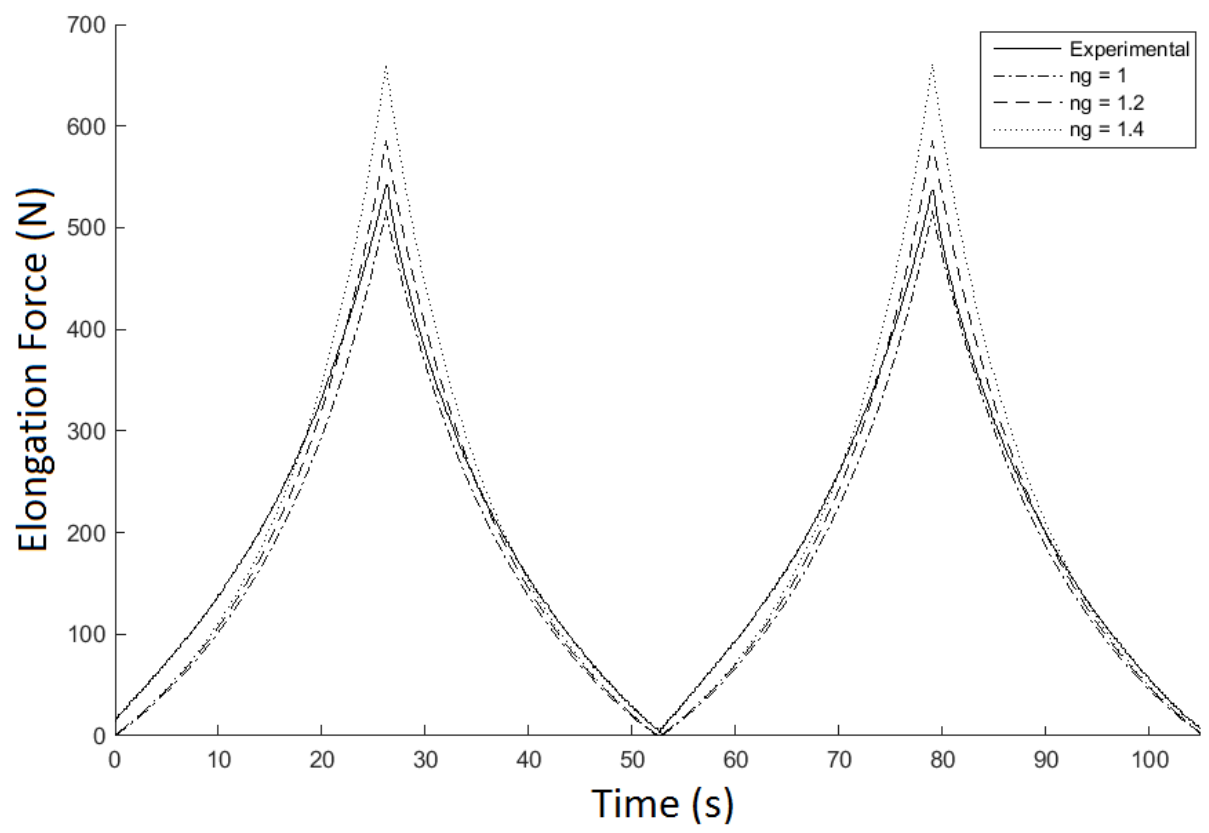

Figure C.5: Force comparison of experimental and simulated results for PAM inflated to $207 \mathrm{kPa}$, tested at $90 \%$ elongation. Bladder stress is not included in this simulation

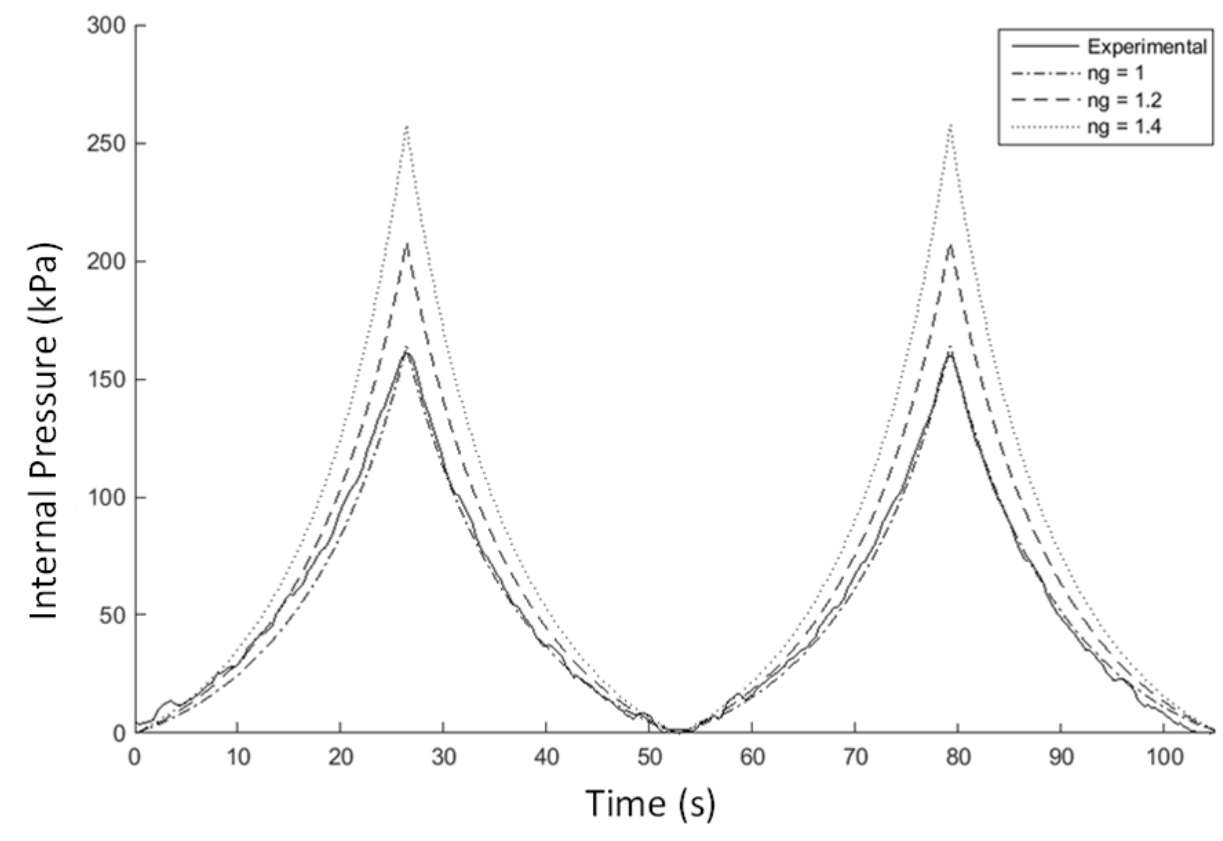

Figure C.6: Pressure comparison of experimental and simulated results for PAM inflated to $207 \mathrm{kPa}$, tested at $90 \%$ elongation. Bladder stress is not included in this simulation 


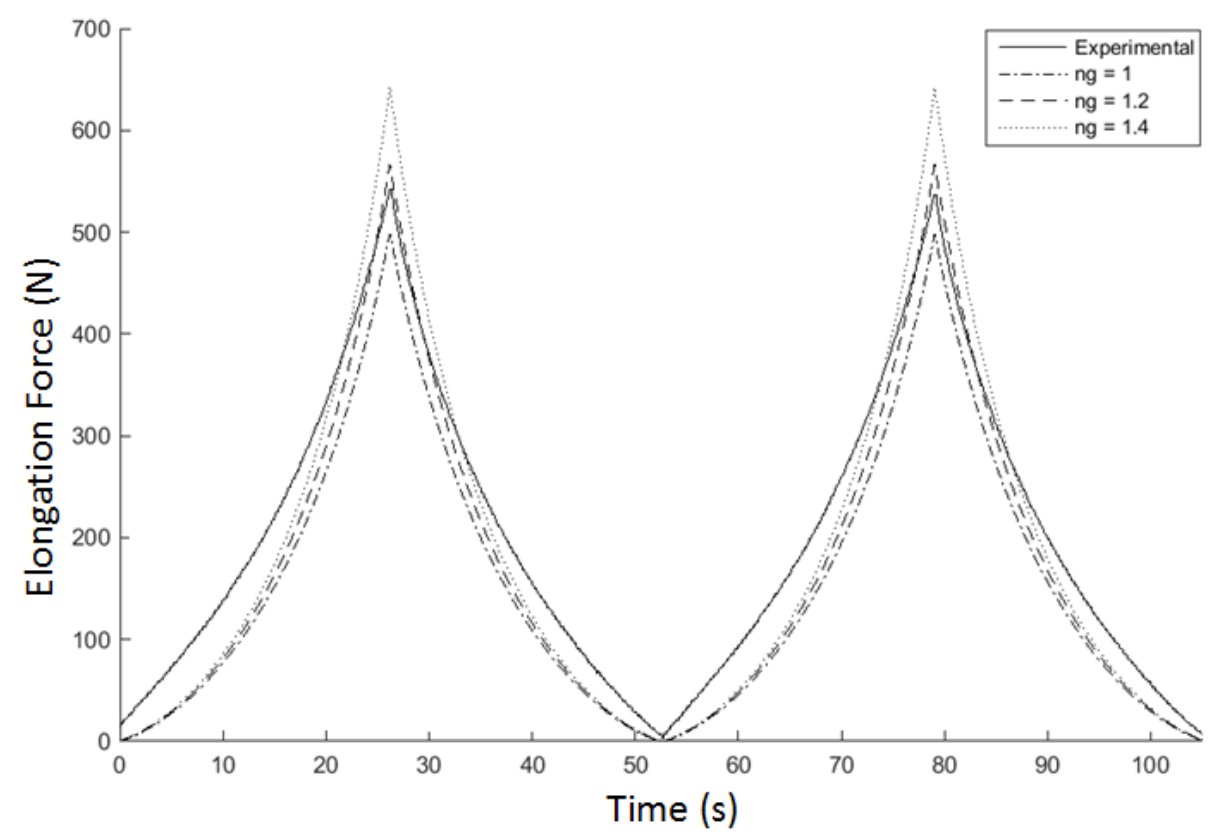

Figure C.7: Force comparison of experimental and simulated results for PAM inflated to $207 \mathrm{kPa}$, tested at $90 \%$ elongation. Bladder stress is indeed included in this simulation

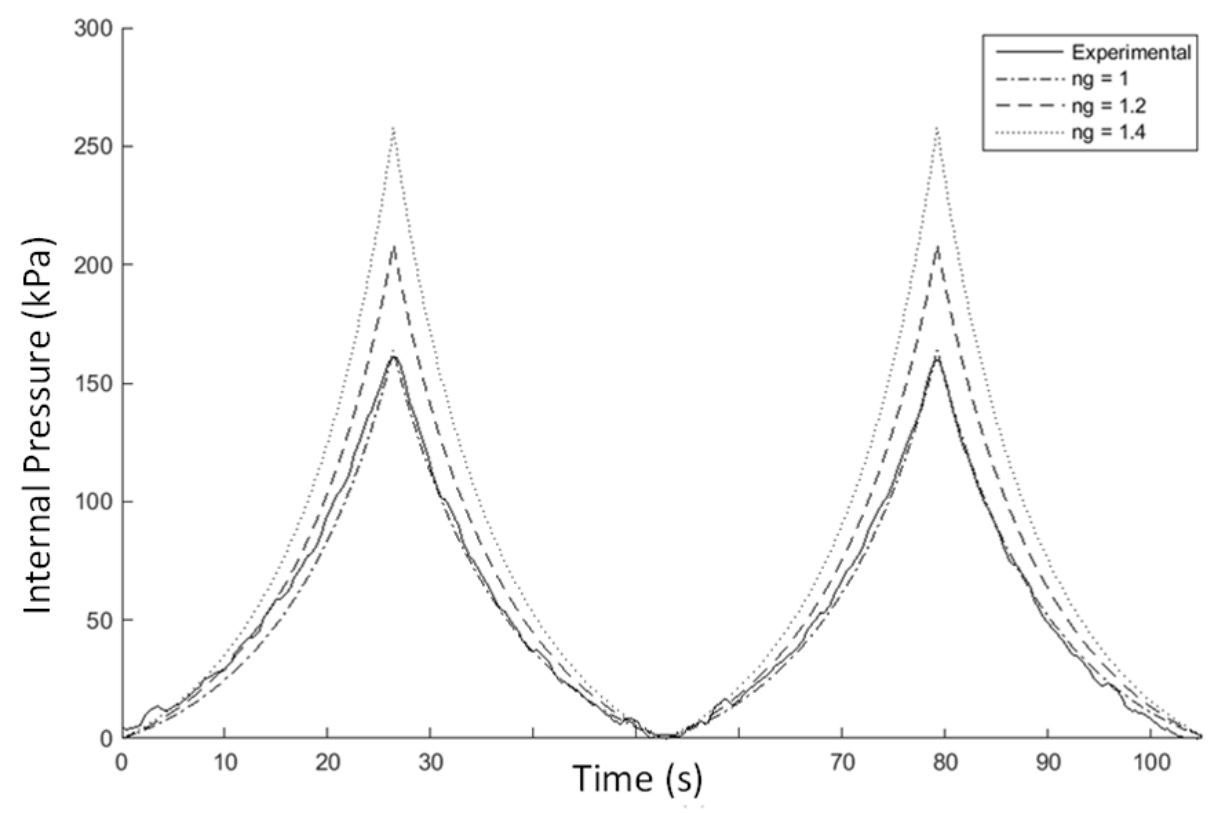

Figure C.8: Pressure comparison of experimental and simulated results for PAM inflated to $207 \mathrm{kPa}$, tested at $90 \%$ elongation. Bladder stress is indeed included in this simulation 


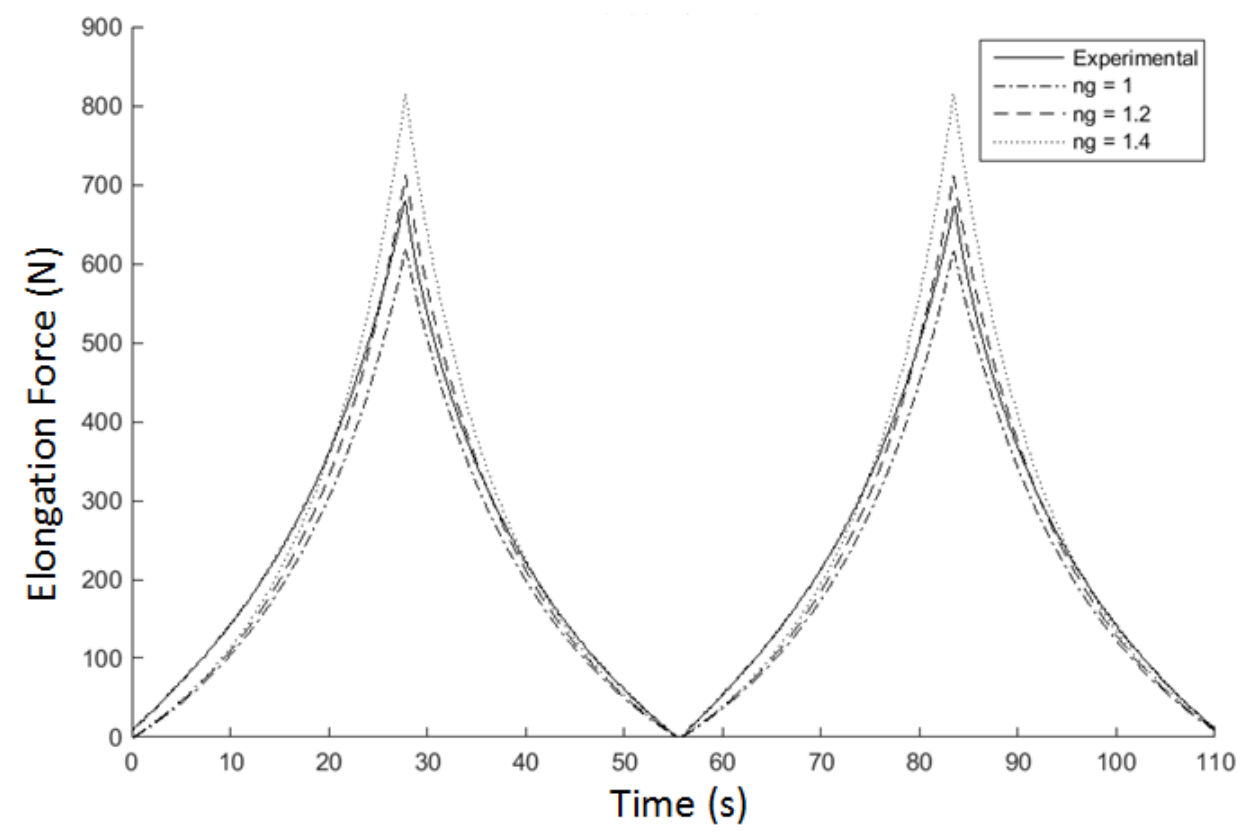

Figure C.9: Force comparison of experimental and simulated results for PAM inflated to $207 \mathrm{kPa}$, tested at $95 \%$ elongation. Bladder stress is not included in this simulation

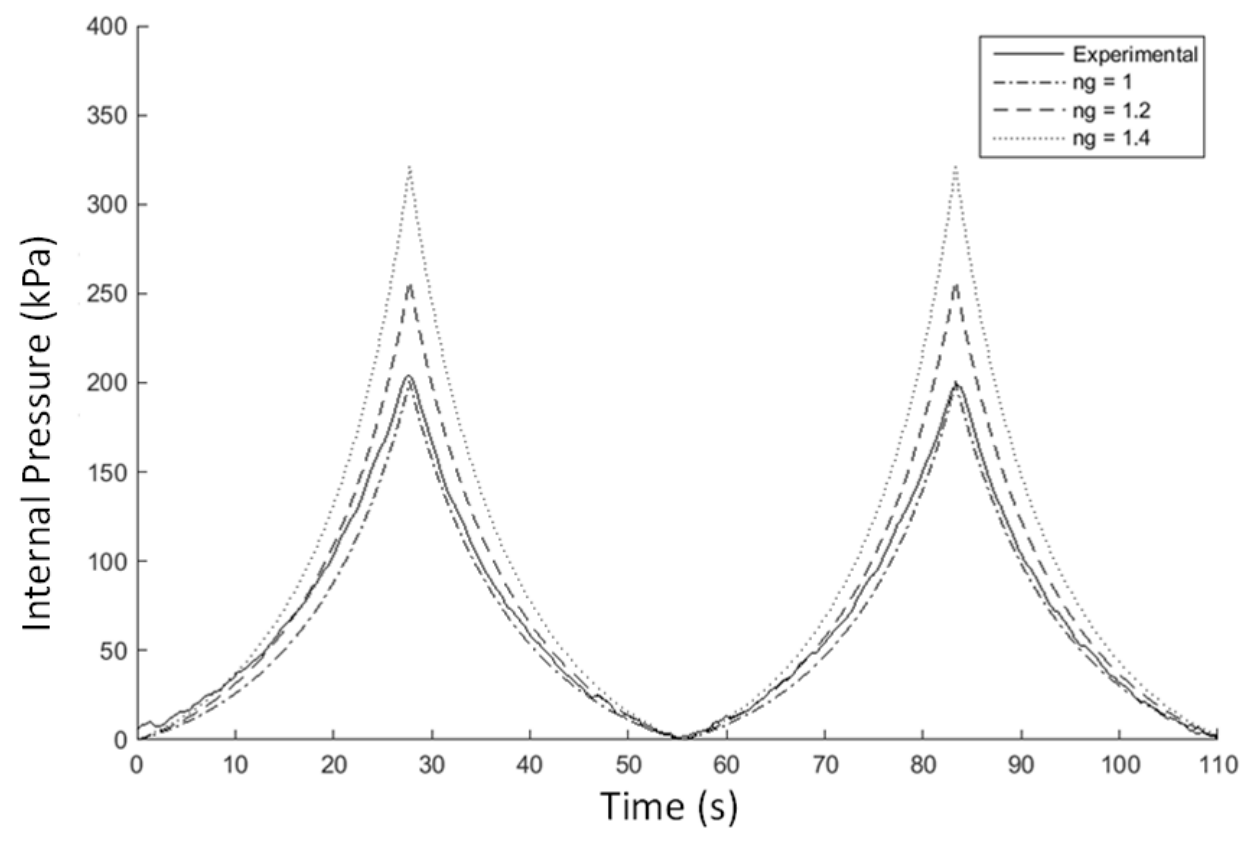

Figure C.10: Pressure comparison of experimental and simulated results for PAM inflated to $207 \mathrm{kPa}$, tested at $95 \%$ elongation. Bladder stress is not included in this simulation 


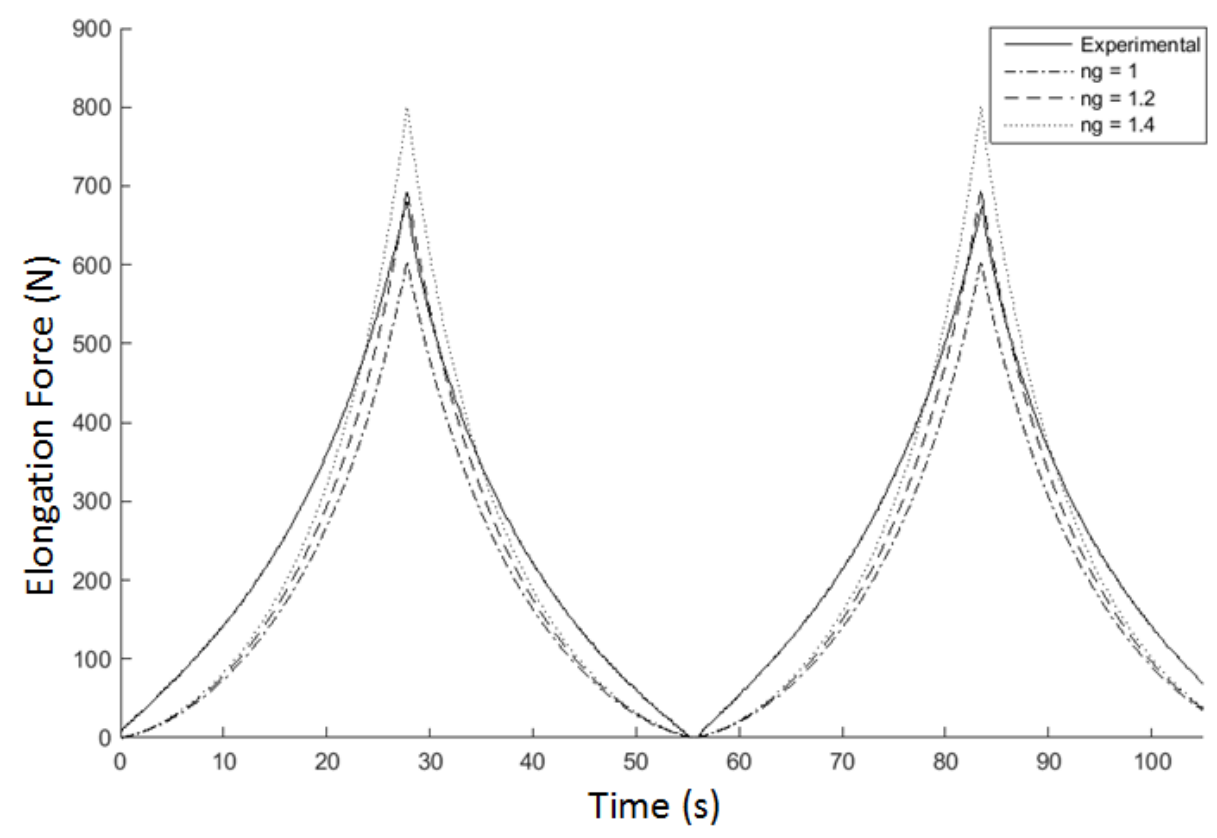

Figure C.11: Force comparison of experimental and simulated results for PAM inflated to $207 \mathrm{kPa}$, tested at $95 \%$ elongation. Bladder stress is indeed included in this simulation

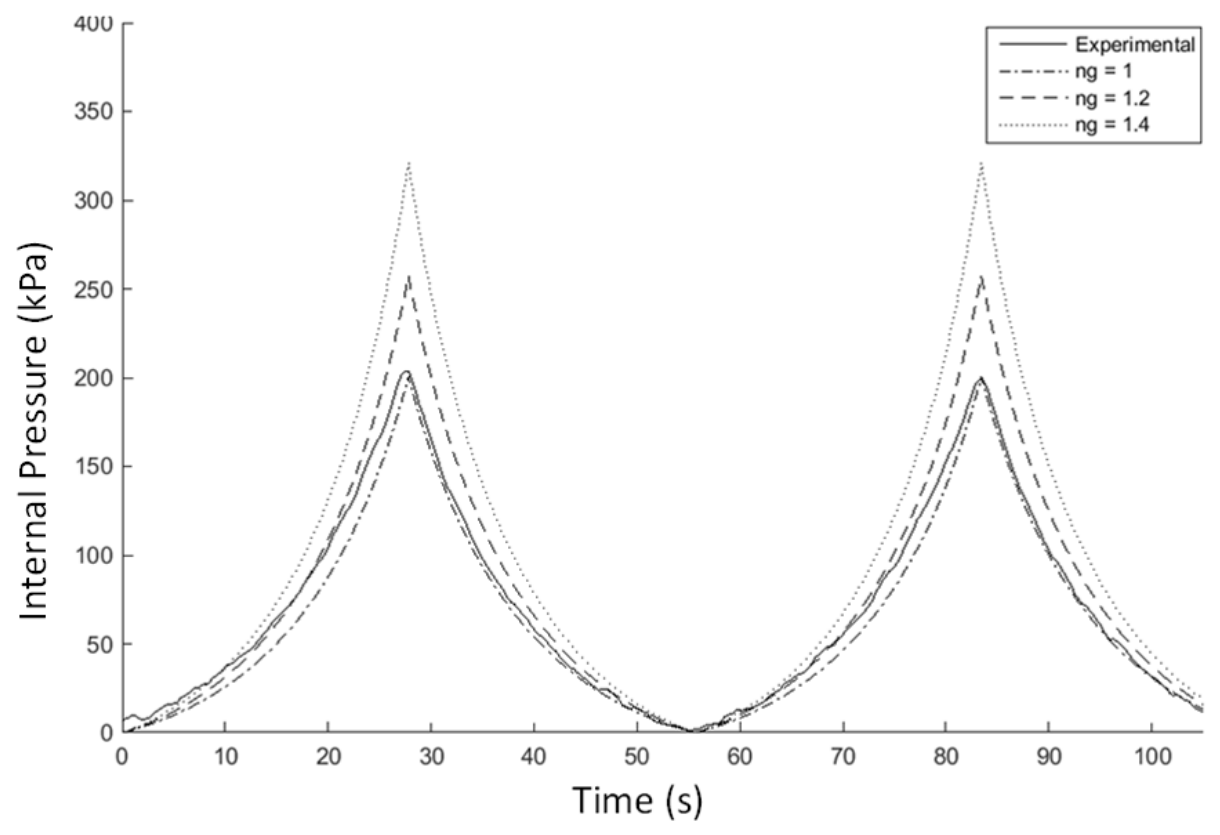

Figure C.12: Pressure comparison of experimental and simulated results for PAM inflated to $207 \mathrm{kPa}$, tested at $95 \%$ elongation. Bladder stress is indeed included in this simulation 


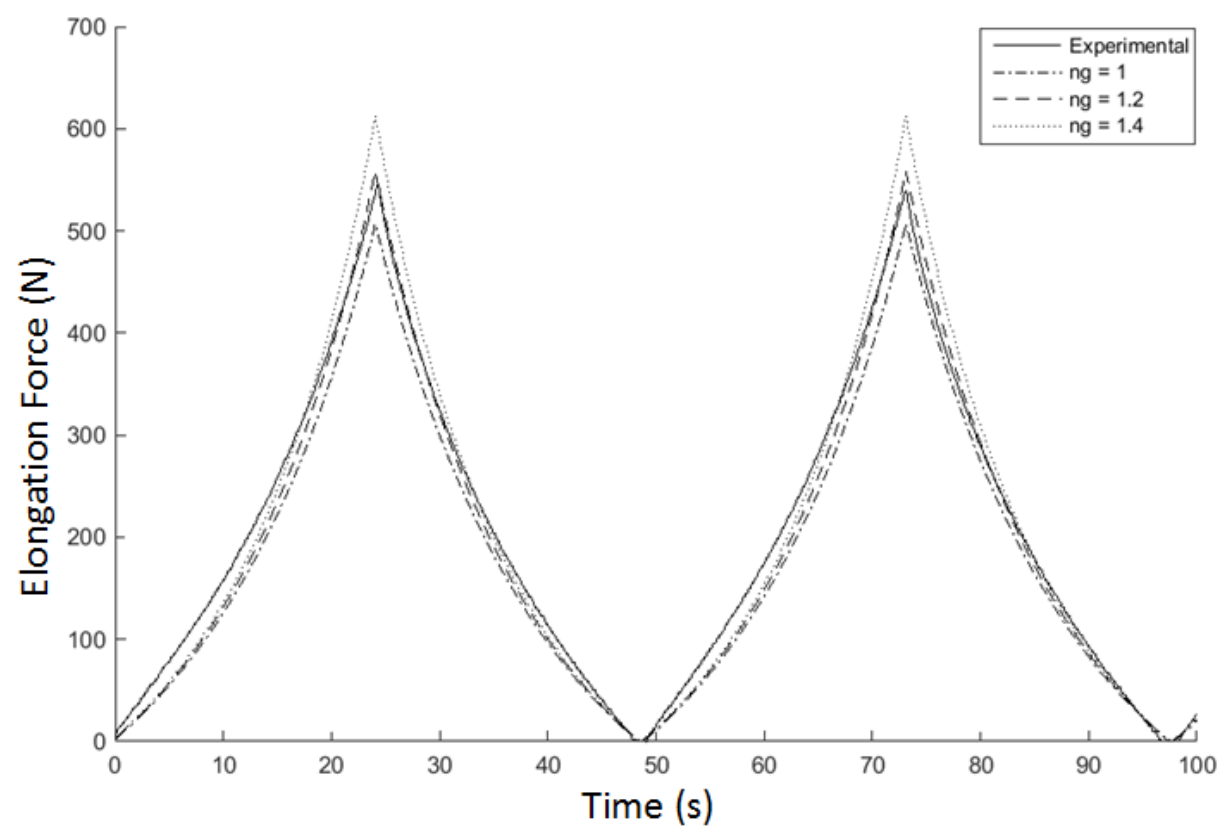

Figure C.13: Force comparison of experimental and simulated results for PAM inflated to $276 \mathrm{kPa}$, tested at $80 \%$ elongation. Bladder stress is not included in this simulation

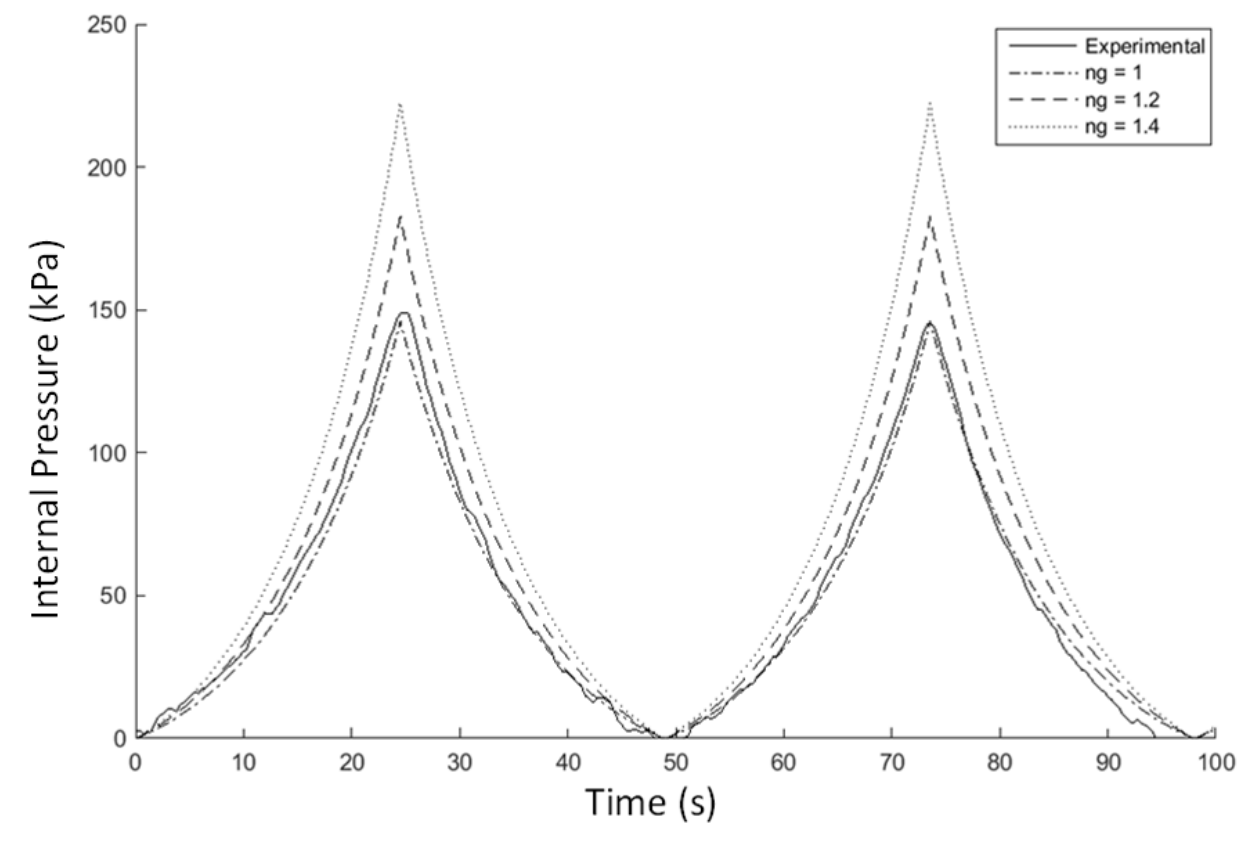

Figure C.14: Pressure comparison of experimental and simulated results for PAM inflated to $276 \mathrm{kPa}$, tested at $80 \%$ elongation. Bladder stress is not included in this simulation 


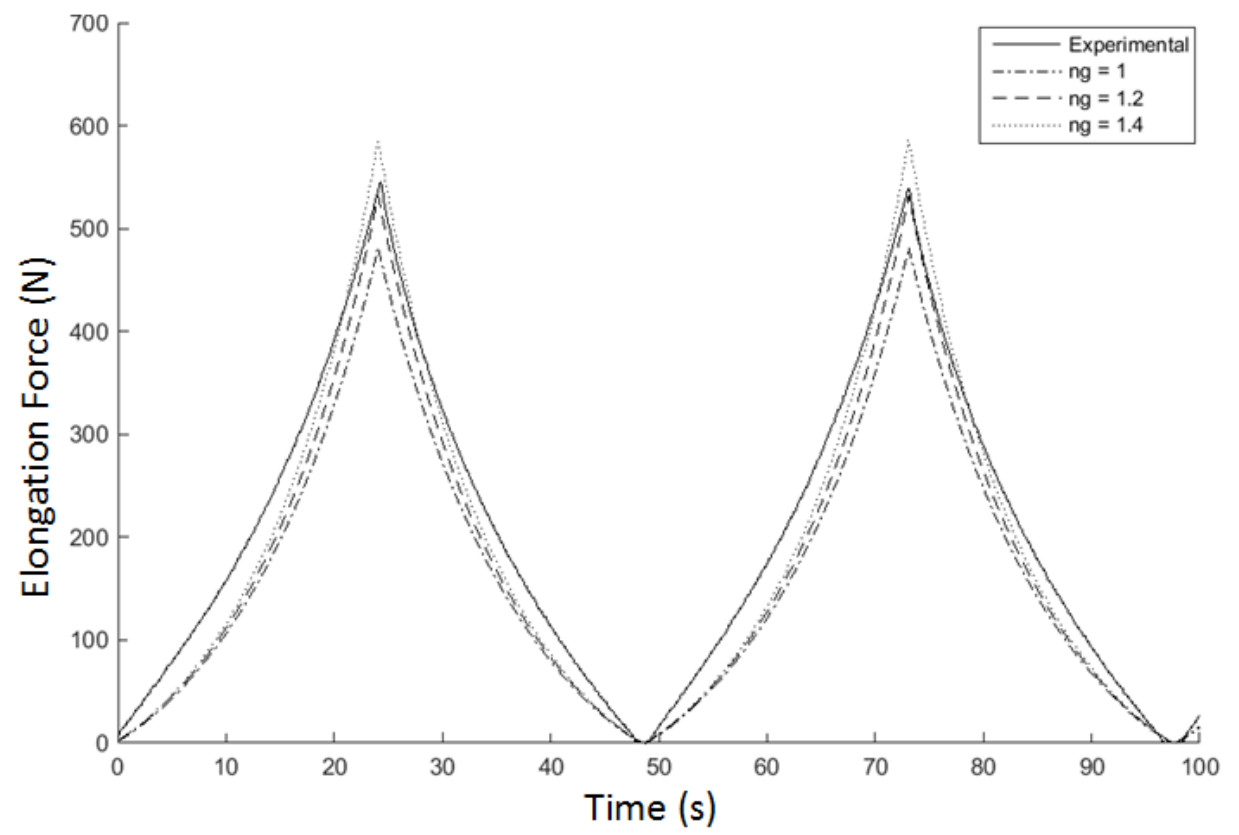

Figure C.15: Force comparison of experimental and simulated results for PAM inflated to $276 \mathrm{kPa}$, tested at $80 \%$ elongation. Bladder stress is indeed included in this simulation

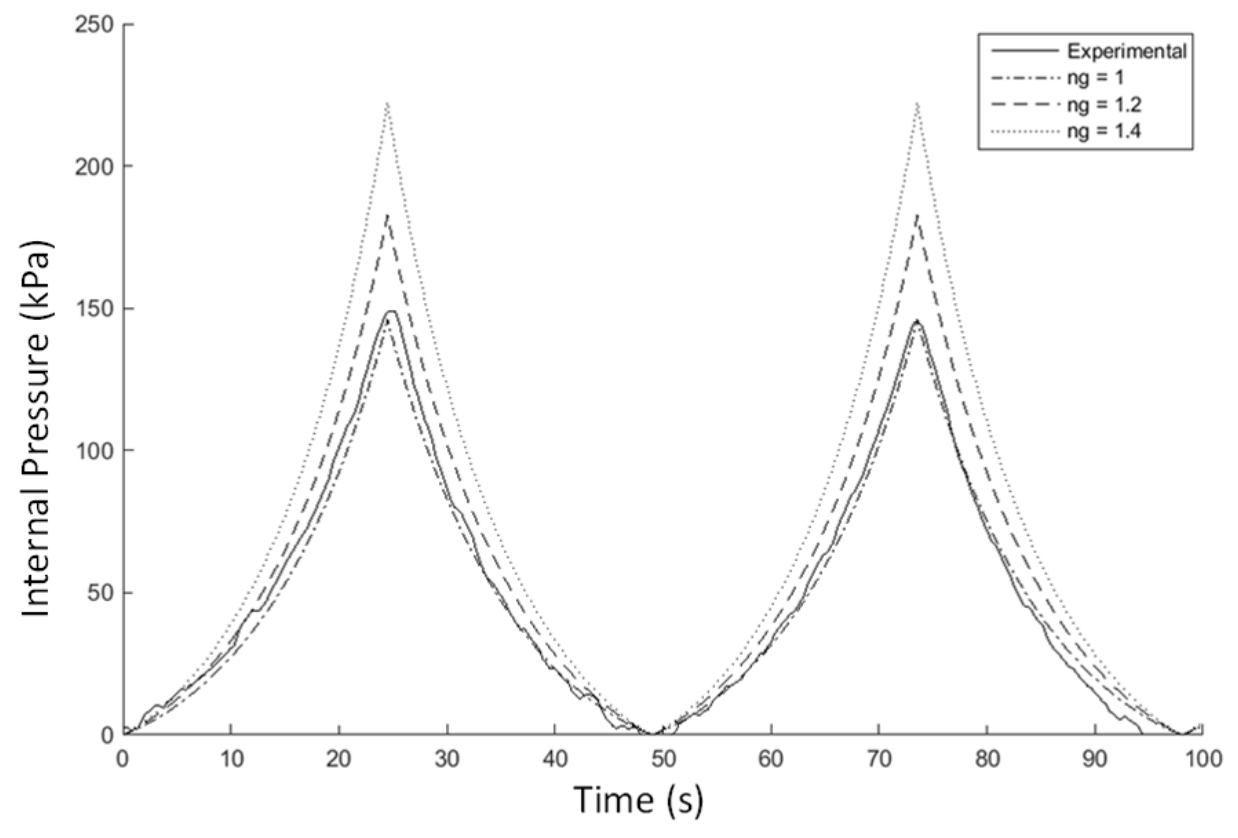

Figure C.16: Pressure comparison of experimental and simulated results for PAM inflated to $276 \mathrm{kPa}$, tested at $80 \%$ elongation. Bladder stress is indeed included in this simulation 


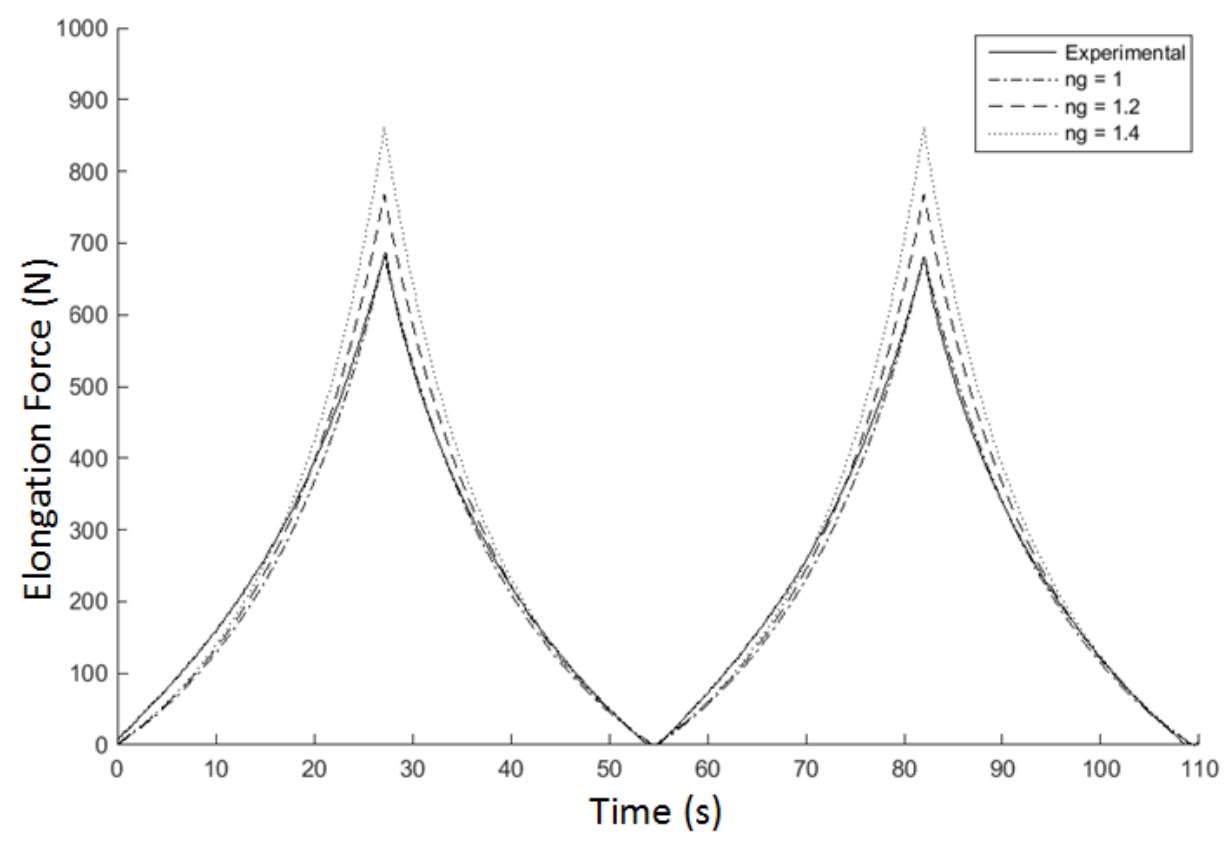

Figure C.17: Force comparison of experimental and simulated results for PAM inflated to $276 \mathrm{kPa}$, tested at $90 \%$ elongation. Bladder stress is not included in this simulation

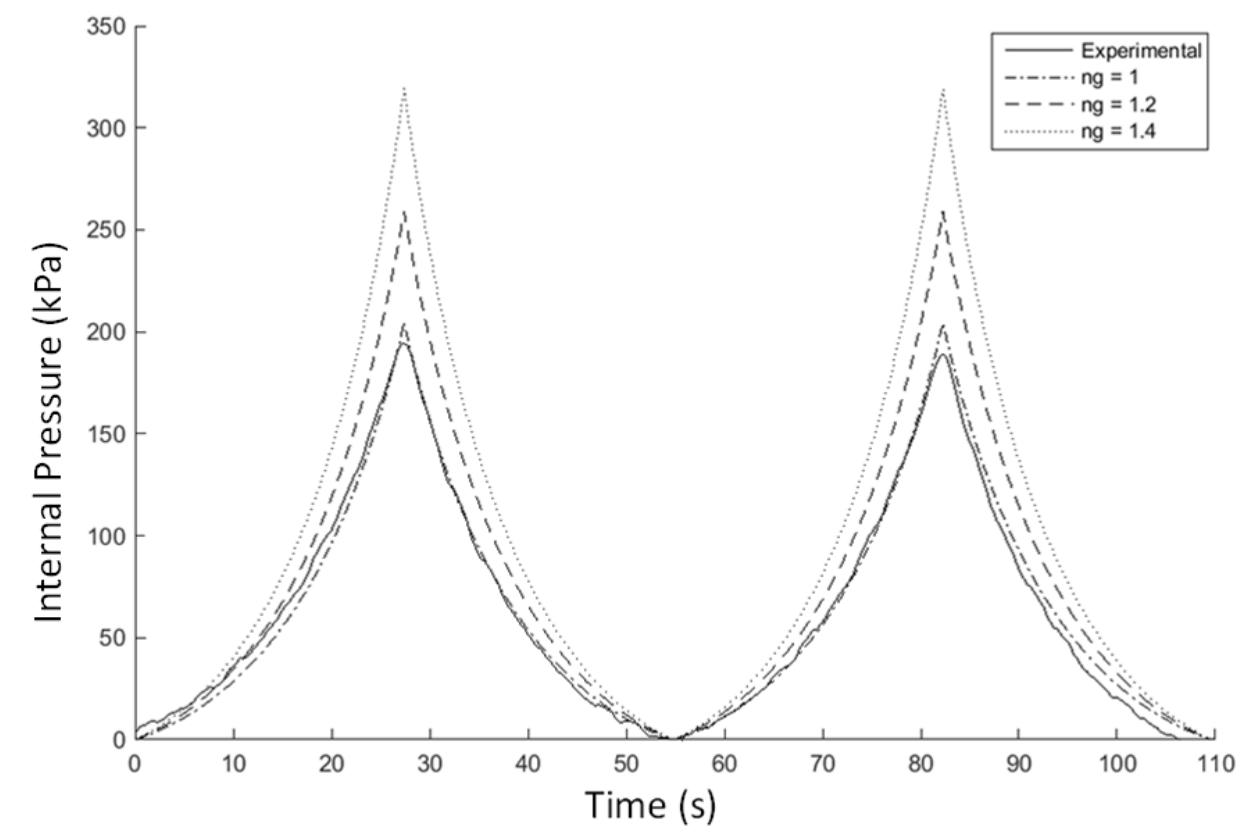

Figure C.18: Pressure comparison of experimental and simulated results for PAM inflated to $276 \mathrm{kPa}$, tested at $90 \%$ elongation. Bladder stress is not included in this simulation 


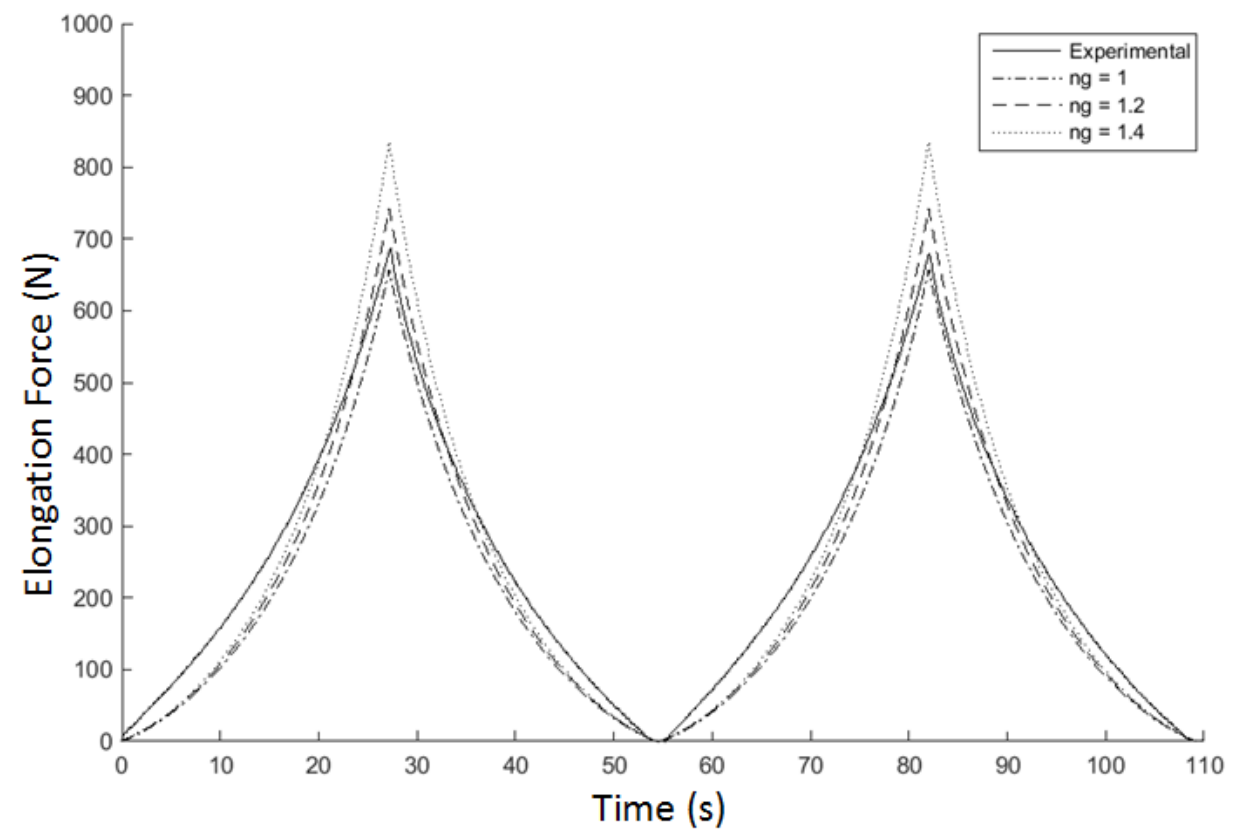

Figure C.19: Force comparison of experimental and simulated results for PAM inflated to $276 \mathrm{kPa}$, tested at $90 \%$ elongation. Bladder stress is indeed included in this simulation

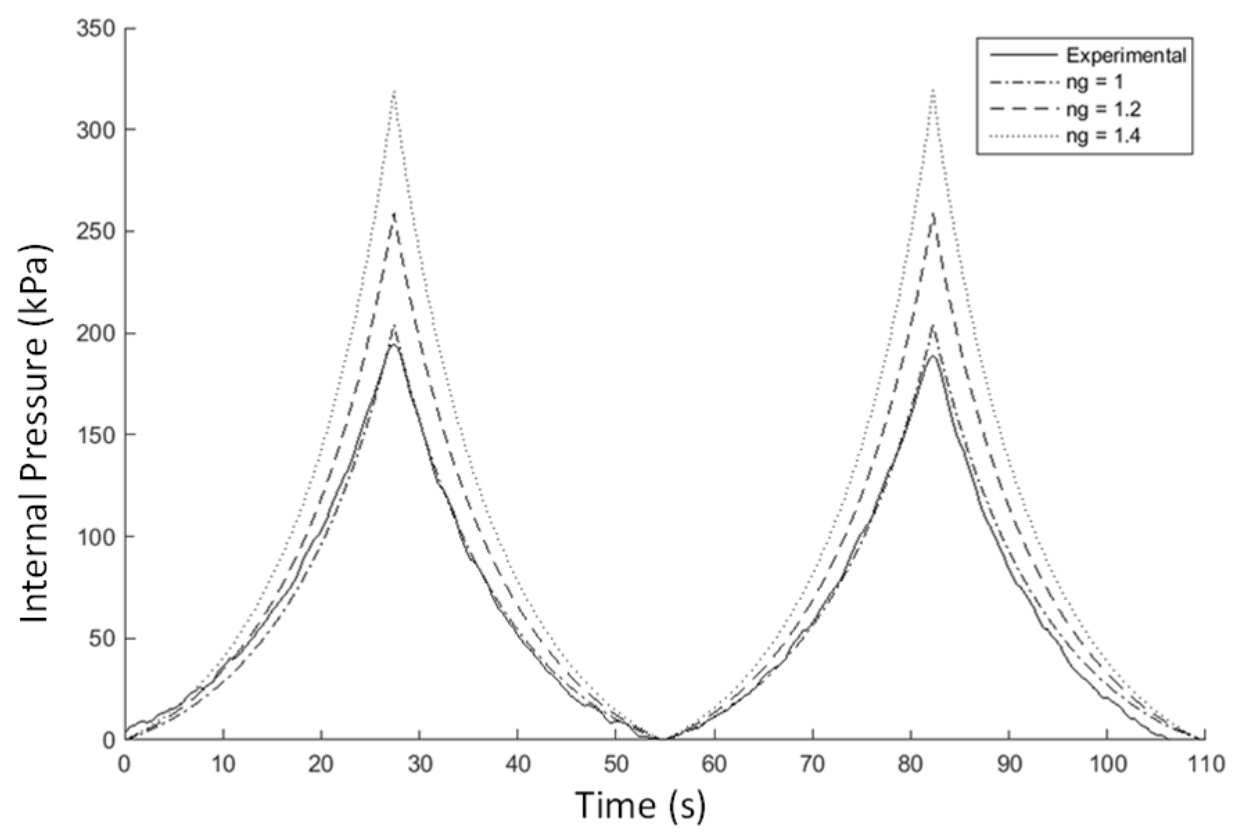

Figure C.20: Pressure comparison of experimental and simulated results for PAM inflated to $276 \mathrm{kPa}$, tested at $90 \%$ elongation. Bladder stress is indeed included in this simulation 


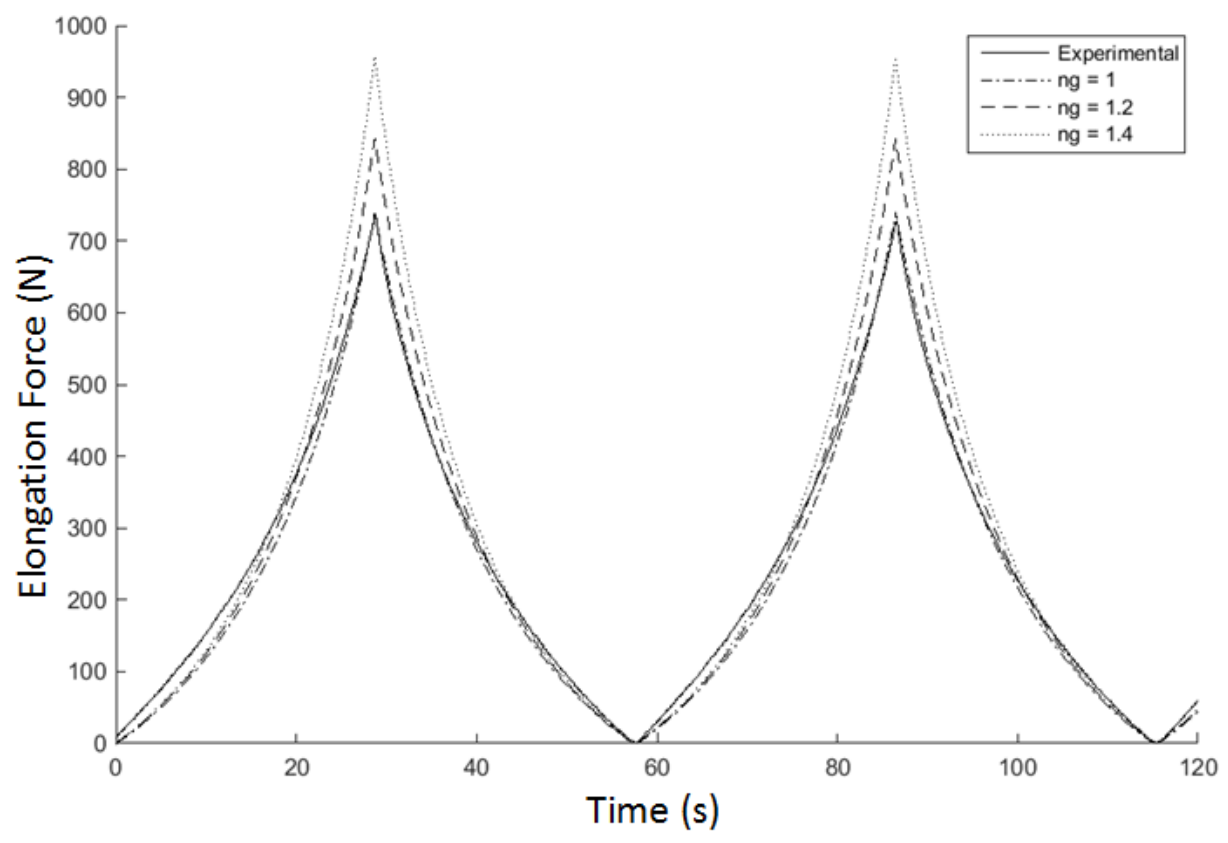

Figure C.21: Force comparison of experimental and simulated results for PAM inflated to $276 \mathrm{kPa}$, tested at $95 \%$ elongation. Bladder stress is not included in this simulation

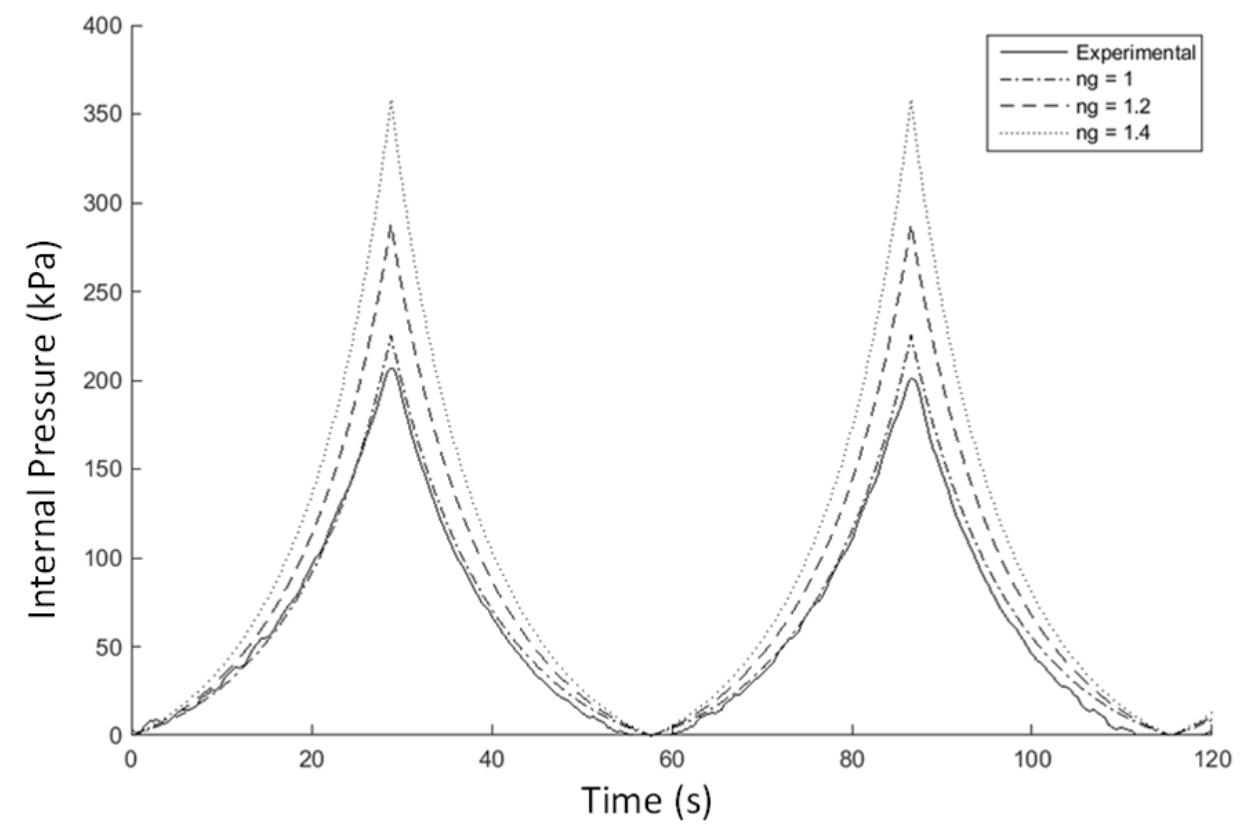

Figure C.22: Pressure comparison of experimental and simulated results for PAM inflated to $276 \mathrm{kPa}$, tested at $95 \%$ elongation. Bladder stress is not included in this simulation 


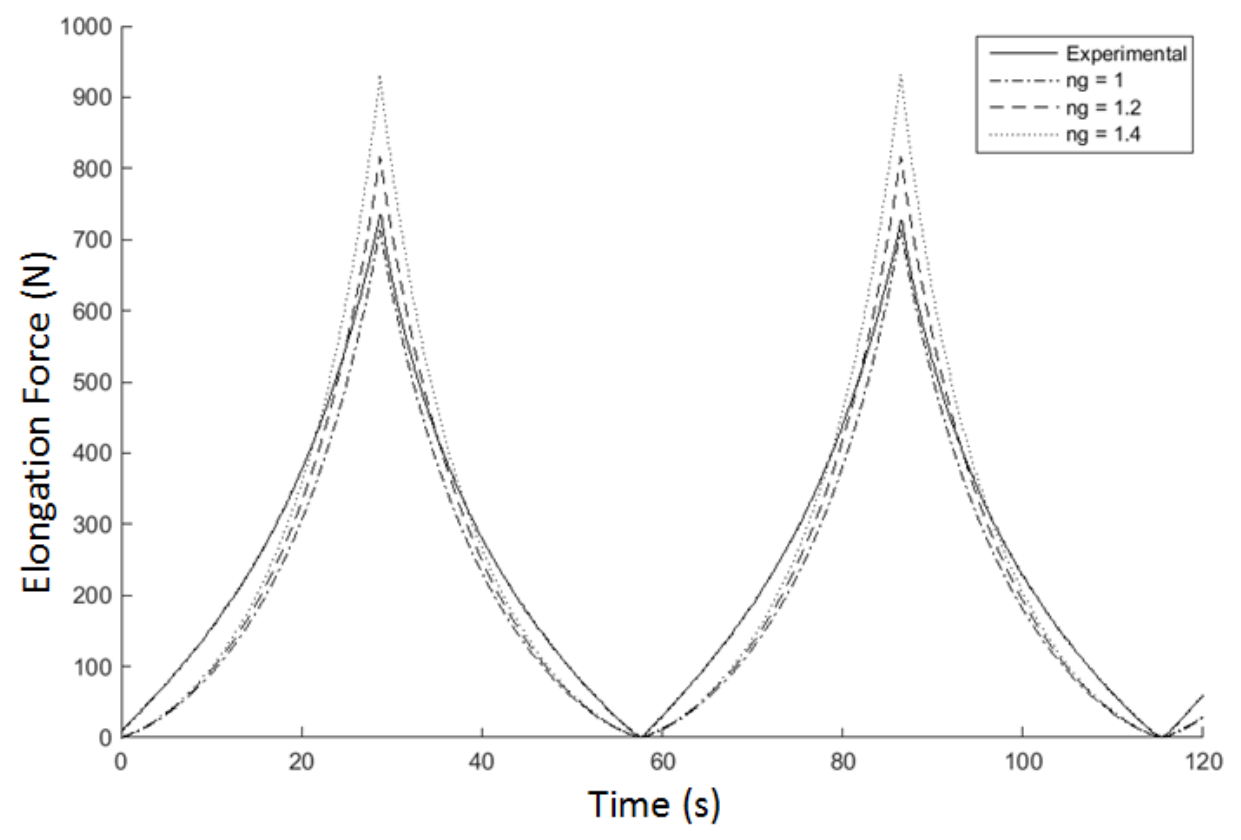

Figure C.23: Force comparison of experimental and simulated results for PAM inflated to $276 \mathrm{kPa}$, tested at $95 \%$ elongation. Bladder stress is indeed included in this simulation

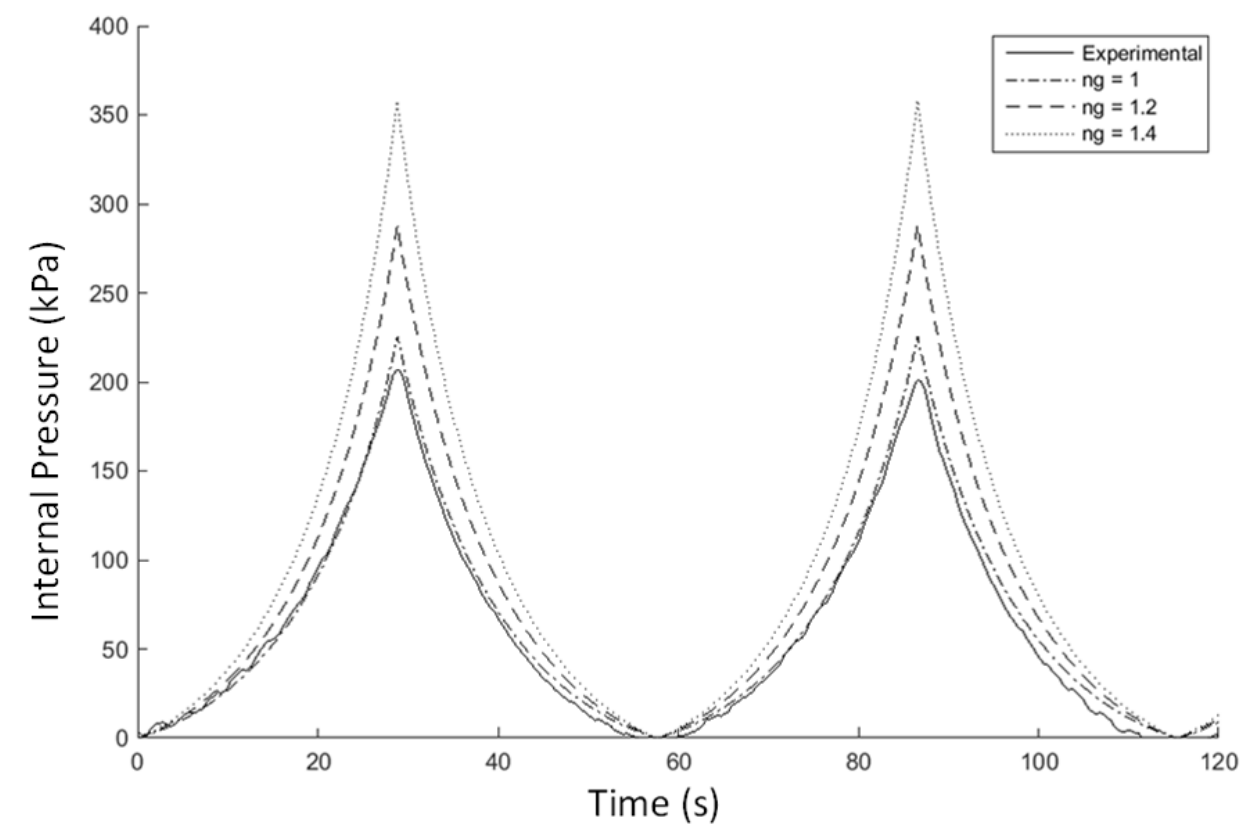

Figure C.24: Pressure comparison of experimental and simulated results for PAM inflated to $276 \mathrm{kPa}$, tested at $95 \%$ elongation. Bladder stress is indeed included in this simulation 


\section{C.3 PAM RMSE results}

Table C.5: RMSE values for low pressure PAM tests

\begin{tabular}{clllllll}
\hline Pressure & \multicolumn{1}{c}{ Model } & \multicolumn{5}{c}{ RMSE value (N / kPa) } \\
\hline \hline \multirow{2}{*}{$35 \mathrm{kPa}$} & \multicolumn{2}{c}{ ng=1 } & \multicolumn{2}{c}{ ng=1.2 } & \multicolumn{2}{c}{ ng=1.4 } \\
\cline { 2 - 8 } & & Force & Pressure & Force & Pressure & Force & Pressure \\
\cline { 2 - 8 } & With Bladder & 63.57 & 20.78 & 55.98 & 15.35 & 48.07 & 9.62 \\
\hline \multirow{2}{*}{$48 \mathrm{kPa}$} & No Bladder & 69.66 & 27.97 & 59.68 & 21.13 & 49.20 & 13.78 \\
\cline { 2 - 8 } & With Bladder & 68.47 & 27.97 & 58.96 & 21.13 & 48.94 & 13.78 \\
\hline \multirow{2}{*}{$55 \mathrm{kPa}$} & No Bladder & 69.30 & 27.36 & 58.62 & 19.92 & 47.47 & 11.96 \\
\cline { 2 - 8 } & With Bladder & 69.54 & 27.36 & 59.42 & 19.92 & 48.84 & 11.95 \\
\hline \multirow{2}{*}{$69 \mathrm{kPa}$} & No Bladder & 76.97 & 28.20 & 63.43 & 18.89 & 49.17 & 8.91 \\
\cline { 2 - 8 } & With Bladder & 78.18 & 28.20 & 65.48 & 18.89 & 52.17 & 8.91 \\
\hline \multirow{2}{*}{$83 \mathrm{kPa}$} & No Bladder & 72.97 & 25.83 & 57.90 & 15.40 & 42.20 & 4.64 \\
\cline { 2 - 8 } & With Bladder & 80.80 & 25.83 & 66.83 & 15.40 & 52.39 & 4.64 \\
\hline \multirow{2}{*}{$104 \mathrm{kPa}$} & No Bladder & 70.62 & 26.02 & 56.07 & 15.68 & 41.08 & 5.46 \\
\cline { 2 - 8 } & With Bladder & 81.27 & 26.02 & 67.73 & 15.68 & 53.74 & 5.46 \\
\hline
\end{tabular}


Table C.6: Difference between peak value of experimental results and simulated results for low pressure PAM tests. Negative value indicates that the peak of the experimental results is lower in magnitude than the simulated results

\begin{tabular}{cllccccc}
\hline \multirow{2}{*}{ Pressure } & \multicolumn{1}{c}{ Model } & \multicolumn{2}{c}{ Difference between peaks (N / kPa) } \\
\hline \hline \multirow{2}{*}{$35 \mathrm{kPa}$} & \multicolumn{2}{c}{ ng=1 } & \multicolumn{2}{c}{ ng=1.2 } & \multicolumn{2}{c}{ ng=1.4 } \\
\cline { 2 - 8 } & & Force & Pressure & Force & Pressure & Force & Pressure \\
\cline { 2 - 8 } & With Bladder & 136.30 & 55.34 & 115.70 & 42.04 & 92.90 & 27.26 \\
\hline \multirow{2}{*}{$48 \mathrm{kPa}$} & No Bladder & 169.30 & 62.23 & 142.40 & 45.21 & 112.7 & 26.13 \\
\cline { 2 - 8 } & With Bladder & 144.20 & 62.23 & 117.60 & 45.21 & 87.80 & 26.13 \\
\hline \multirow{2}{*}{$55 \mathrm{kPa}$} & No Bladder & 169.70 & 59.23 & 140.30 & 40.69 & 107.80 & 19.90 \\
\cline { 2 - 8 } & With Bladder & 146.40 & 59.23 & 117.30 & 40.69 & 84.80 & 19.90 \\
\hline \multirow{2}{*}{$69 \mathrm{kPa}$} & No Bladder & 190.60 & 61.60 & 152.90 & 38.1 & 110.80 & 11.60 \\
\cline { 2 - 8 } & With Bladder & 163.20 & 61.60 & 125.90 & 38.10 & 83.80 & 11.60 \\
\hline \multirow{2}{*}{$83 \mathrm{kPa}$} & No Bladder & 186.40 & 59.38 & 142.60 & 32.10 & 93.70 & 1.30 \\
\cline { 2 - 8 } & With Bladder & 171.80 & 59.38 & 128.50 & 32.10 & 79.60 & 1.30 \\
\hline \multirow{2}{*}{$104 \mathrm{kPa}$} & No Bladder & 167.60 & 59.48 & 123.80 & 32.20 & 74.90 & 1.40 \\
\cline { 2 - 8 } & With Bladder & 153.00 & 59.48 & 109.70 & 32.20 & 60.80 & 1.40 \\
\hline
\end{tabular}


Table C.7: RMSE values for high pressure PAM tests with and without the bladder stress model

\begin{tabular}{|c|c|c|c|c|c|c|c|c|}
\hline \multirow[t]{3}{*}{ Pressure } & \multirow[t]{3}{*}{ Stretch } & \multirow[t]{3}{*}{ Model } & \multicolumn{6}{|c|}{ RMSE value } \\
\hline & & & \multicolumn{2}{|c|}{$n g=1$} & \multicolumn{2}{|c|}{$\mathrm{ng}=1.2$} & \multicolumn{2}{|c|}{$\mathrm{ng}=1.4$} \\
\hline & & & $\mathrm{F}(\mathrm{N})$ & $\mathrm{P}(\mathrm{kPa})$ & $\mathrm{F}(\mathrm{N})$ & $\mathrm{P}(\mathrm{kPa})$ & $\mathrm{F}(\mathrm{N})$ & $\mathrm{P}(\mathrm{kPa})$ \\
\hline \multirow{6}{*}{$138 \mathrm{kPa}$} & \multirow{2}{*}{$80 \%$} & No Bl & 59.95 & 17.29 & 41.61 & 6.66 & 31.61 & 5.47 \\
\hline & & With Bl & 79.41 & 20.01 & 71.45 & 12.78 & 63.49 & 5.76 \\
\hline & \multirow{2}{*}{$90 \%$} & No Bl & 92.15 & 32.66 & 80.94 & 23.90 & 69.58 & 14.68 \\
\hline & & With Bl & 110.02 & 32.66 & 99.54 & 23.90 & 88.57 & 14.68 \\
\hline & \multirow{2}{*}{$95 \%$} & No Bl & 104.17 & 35.59 & 89.27 & 24.56 & 99.07 & 22.54 \\
\hline & & With Bl & 123.53 & 35.56 & 109.65 & 24.56 & 95.03 & 12.95 \\
\hline \multirow{6}{*}{$207 \mathrm{kPa}$} & \multirow{2}{*}{$80 \%$} & No Bl & 10.03 & 18.27 & 20.23 & 30.35 & 34.47 & 43.21 \\
\hline & & With Bl & 21.26 & 18.27 & 17.00 & 30.35 & 24.29 & 43.21 \\
\hline & \multirow{2}{*}{$90 \%$} & No Bl & 25.06 & 25.87 & 20.82 & 43.88 & 40.45 & 63.42 \\
\hline & & With Bl & 49.09 & 25.87 & 36.39 & 43.88 & 39.57 & 63.42 \\
\hline & \multirow{2}{*}{$95 \%$} & No Bl & 34.32 & 24.08 & 20.19 & 45.11 & 42.47 & 68.21 \\
\hline & & With Bl & 61.33 & 24.08 & 43.27 & 45.11 & 45.10 & 68.21 \\
\hline \multirow{6}{*}{$276 \mathrm{kPa}$} & \multirow{2}{*}{$80 \%$} & No Bl & 27.43 & 17.67 & 16.85 & 31.48 & 20.82 & 46.16 \\
\hline & & With Bl & 47.86 & 17.67 & 35.26 & 31.48 & 28.24 & 46.16 \\
\hline & \multirow{2}{*}{$90 \%$} & No Bl & 16.78 & 30.00 & 29.32 & 50.99 & 57.98 & 73.64 \\
\hline & & With Bl & 40.27 & 30.00 & 31.63 & 50.99 & 47.42 & 73.64 \\
\hline & \multirow{2}{*}{$95 \%$} & No Bl & 21.19 & 34.73 & 37.54 & 58.05 & 71.53 & 83.47 \\
\hline & & With Bl & 47.15 & 34.73 & 40.81 & 58.05 & 61.3 & 83.47 \\
\hline
\end{tabular}


Table C.8: Difference between peak value of experimental results and simulated results for lhigh pressure PAM tests. Negative value indicates that the peak of the experimental results is lower in magnitude than the simulated results

\begin{tabular}{|c|c|c|c|c|c|c|c|c|}
\hline \multirow[t]{3}{*}{ Pressure } & \multirow[t]{3}{*}{ Stretch } & \multirow[t]{3}{*}{ Model } & \multicolumn{6}{|c|}{ RMSE value } \\
\hline & & & \multicolumn{2}{|c|}{$\mathrm{ng}=1$} & \multicolumn{2}{|c|}{$\mathrm{ng}=1.2$} & \multicolumn{2}{|c|}{$\mathrm{ng}=1.4$} \\
\hline & & & $\mathrm{F}(\mathrm{N})$ & $\mathrm{P}(\mathrm{kPa})$ & $\mathrm{F}(\mathrm{N})$ & $\mathrm{P}(\mathrm{kPa})$ & $\mathrm{F}(\mathrm{N})$ & $\mathrm{P}(\mathrm{kPa})$ \\
\hline \multirow{6}{*}{$138 \mathrm{kPa}$} & \multirow{2}{*}{$80 \%$} & No Bl & 126.70 & 38.31 & 76.90 & 10.40 & 43.30 & -13.80 \\
\hline & & With Bl & 155.40 & 45.16 & 131.50 & 26.85 & 106.00 & 7.10 \\
\hline & \multirow{2}{*}{$90 \%$} & No Bl & 213.00 & 71.14 & 181.40 & 49.40 & 147.20 & 25.50 \\
\hline & & With Bl & 227.50 & 71.14 & 196.40 & 49.40 & 162.20 & 25.50 \\
\hline & \multirow{2}{*}{$95 \%$} & No Bl & 253.30 & 81.30 & 241.90 & 52.40 & 208.90 & 54.30 \\
\hline & & With Bl & 264.60 & 81.30 & 220.90 & 52.40 & 172.10 & 20.30 \\
\hline \multirow{6}{*}{$207 \mathrm{kPa}$} & \multirow{2}{*}{$80 \%$} & No Bl & -7.40 & -41.75 & -46.10 & -69.75 & -87.20 & -100.15 \\
\hline & & With Bl & 12.50 & -41.75 & -25.90 & -69.75 & -67.00 & -100.15 \\
\hline & \multirow{2}{*}{$90 \%$} & No Bl & 26.10 & -61.70 & -43.10 & -106.20 & -118.80 & -155.60 \\
\hline & & With Bl & 43.50 & -61.70 & -25.00 & -106.20 & -110.80 & -155.60 \\
\hline & \multirow{2}{*}{$95 \%$} & No Bl & 60.30 & -67.20 & -32.80 & -123.90 & $-136.70-$ & 187.70 \\
\hline & & With Bl & 73.90 & -67.20 & -18.40 & -123.90 & -122.20 & -187.70 \\
\hline \multirow{6}{*}{$276 \mathrm{kPa}$} & \multirow{2}{*}{$80 \%$} & No Bl & 37.50 & -48.46 & -13.80 & -85.16 & -68.00 & -124.76 \\
\hline & & With Bl & 63.20 & -48.46 & 12.30 & -85.16 & -41.90 & -124.76 \\
\hline & \multirow{2}{*}{$90 \%$} & No Bl & 3.90 & -77.10 & -81.90 & -131.60 & -175.50 & -191.90 \\
\hline & & With Bl & 29.90 & -77.10 & -55.40 & -131.60 & -148.90 & -191.90 \\
\hline & \multirow{2}{*}{$95 \%$} & No Bl & -3.30 & -90.20 & -107.00 & -152.70 & -221.90 & -222.60 \\
\hline & & With Bl & 21.30 & -90.20 & -81.70 & -152.70 & -196.50 & -222.60 \\
\hline
\end{tabular}




\section{Appendix D}

\section{Mechanical testing results}

\section{D.1 Comparison of $25 \%$ of ideal spring force vs PAM}

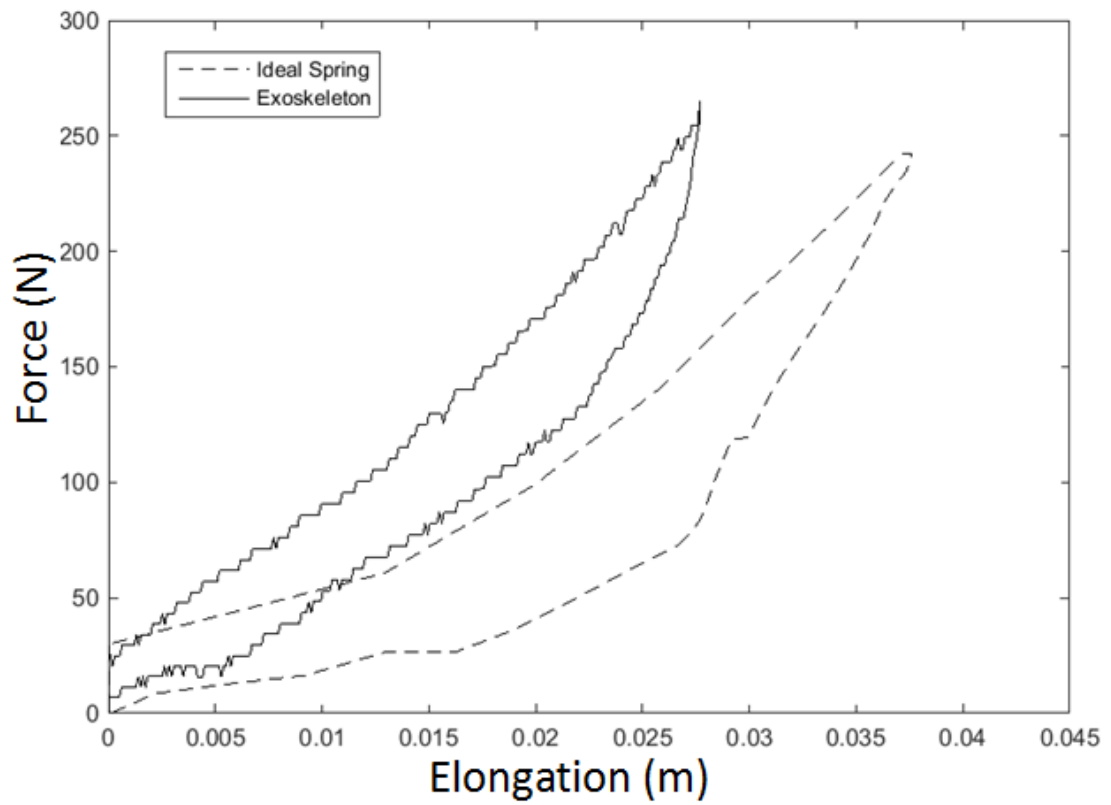

Figure D.1: Mechanical testing results of the force vs elongation with the PAM inflated to $70 \mathrm{kPa}$ and with a vertical displacement of $5 \mathrm{~cm}$ 


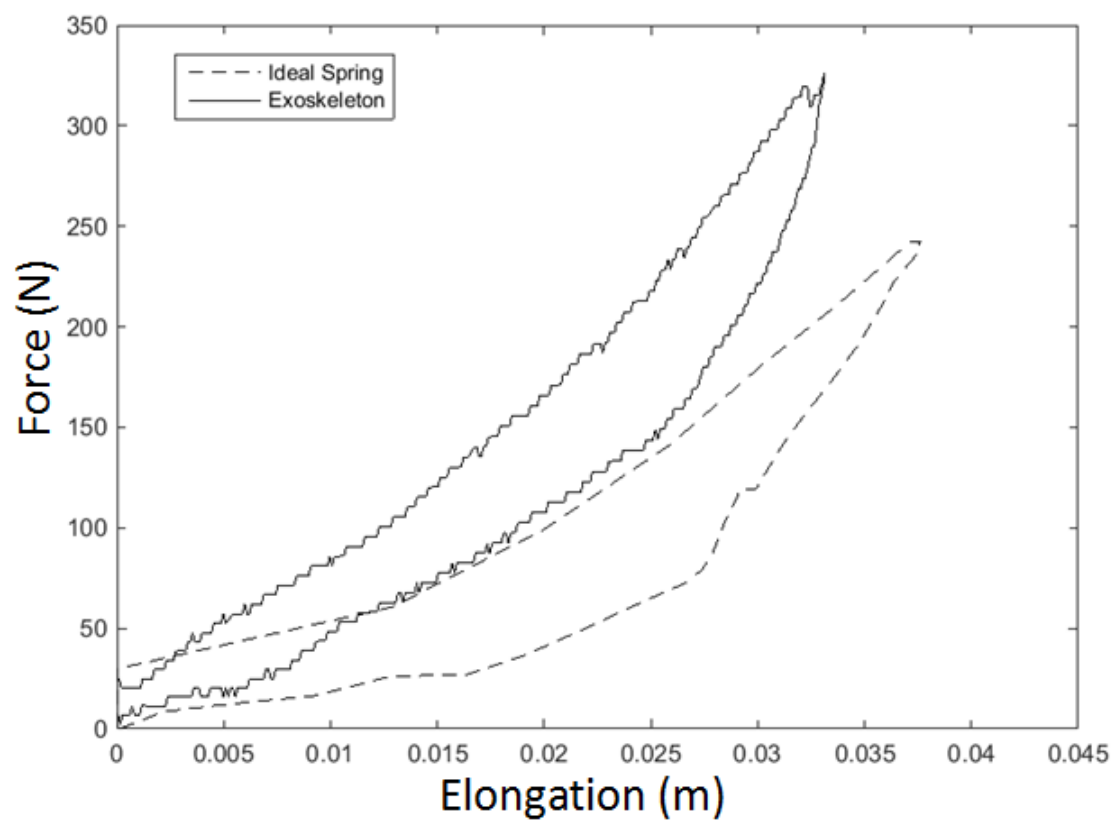

Figure D.2: Mechanical testing results of the force vs elongation with the PAM inflated to $70 \mathrm{kPa}$ and with a vertical displacement of $6 \mathrm{~cm}$

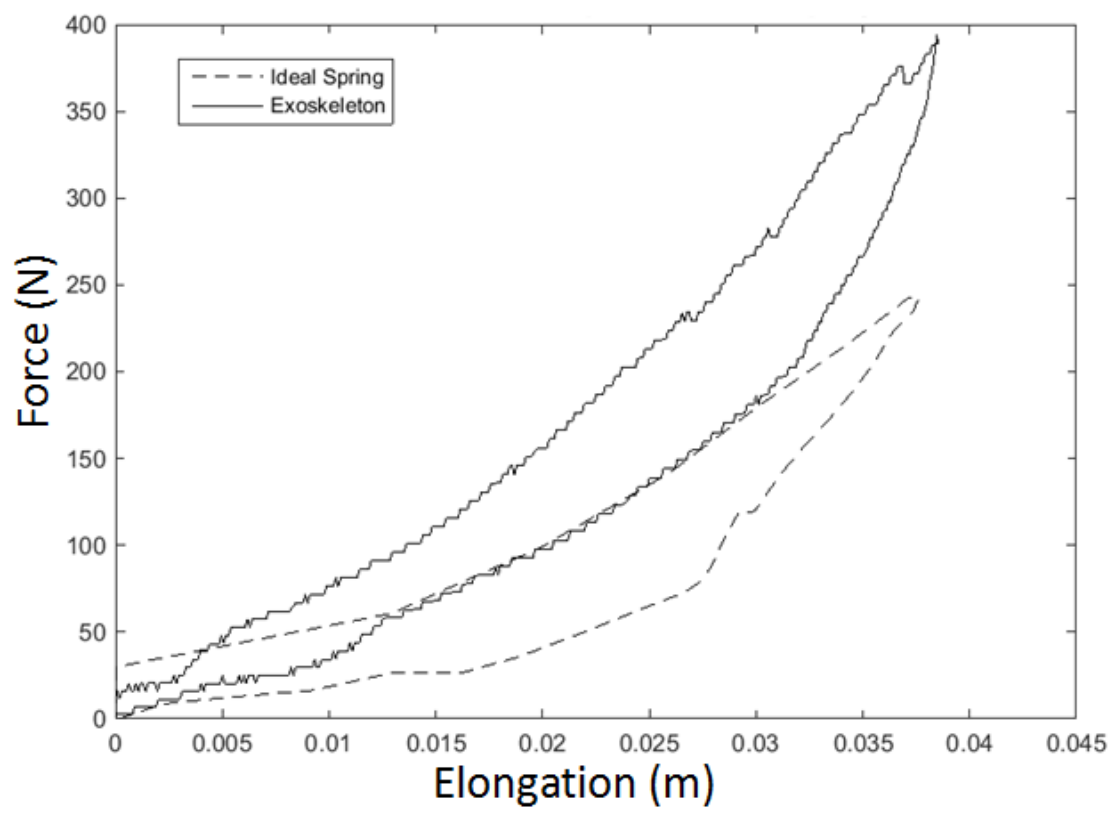

Figure D.3: Mechanical testing results of the force vs elongation with the PAM inflated to $55 \mathrm{kPa}$ and with a vertical displacement of $7 \mathrm{~cm}$ 


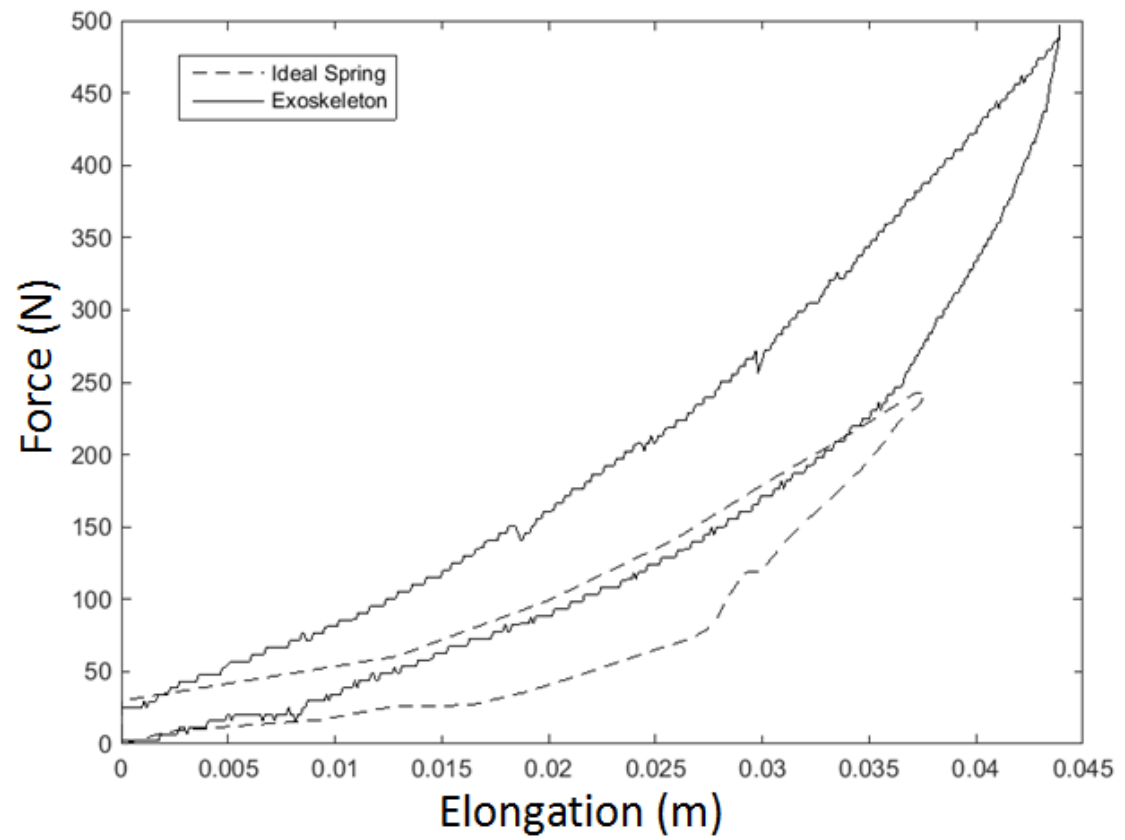

Figure D.4: Mechanical testing results of the force vs elongation with the PAM inflated to $48 \mathrm{kPa}$ and with a vertical displacement of $8 \mathrm{~cm}$ 


\section{D.2 Comparison of ideal spring force vs PAM, with percentage of ideal spring adjusted to fit PAM}

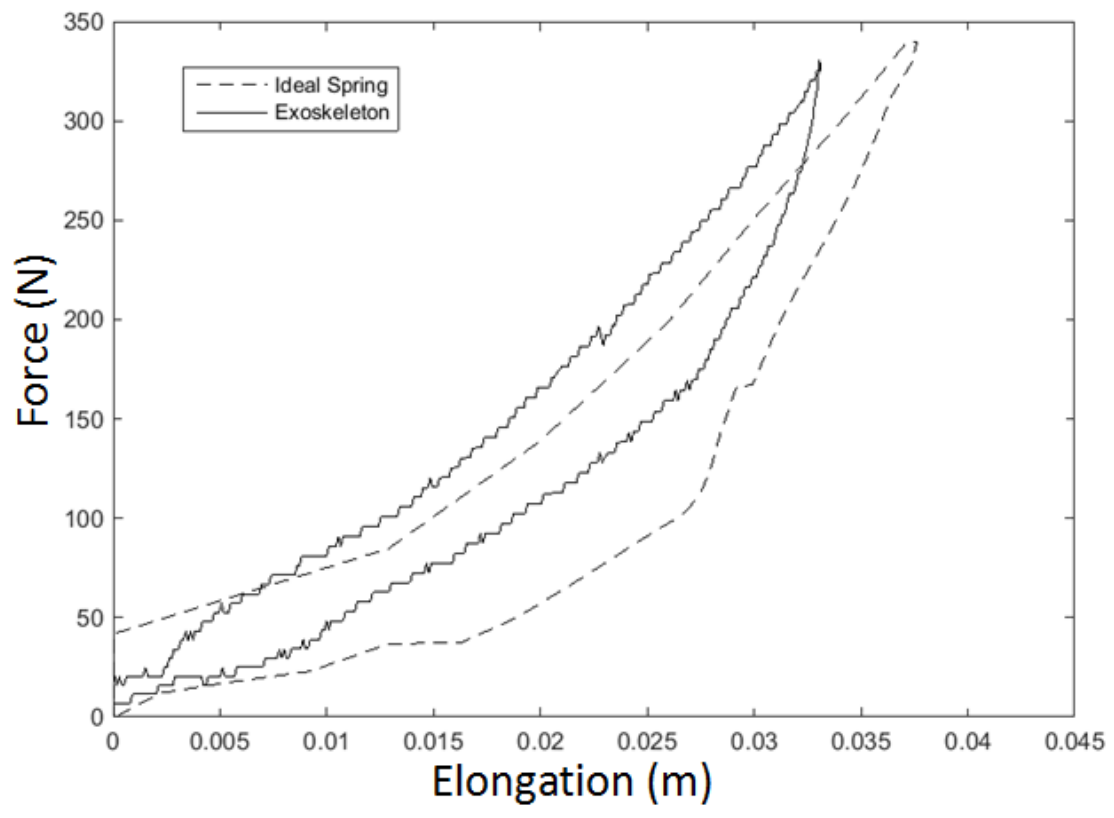

Figure D.5: Mechanical testing results of the ideal spring force vs elongation with the PAM inflated to $70 \mathrm{kPa}$ and with a vertical displacement of $6 \mathrm{~cm}$. The percentage of the ideal spring force was fitted to match the PAM force. The ideal spring force in this case is set to $35 \%$ 


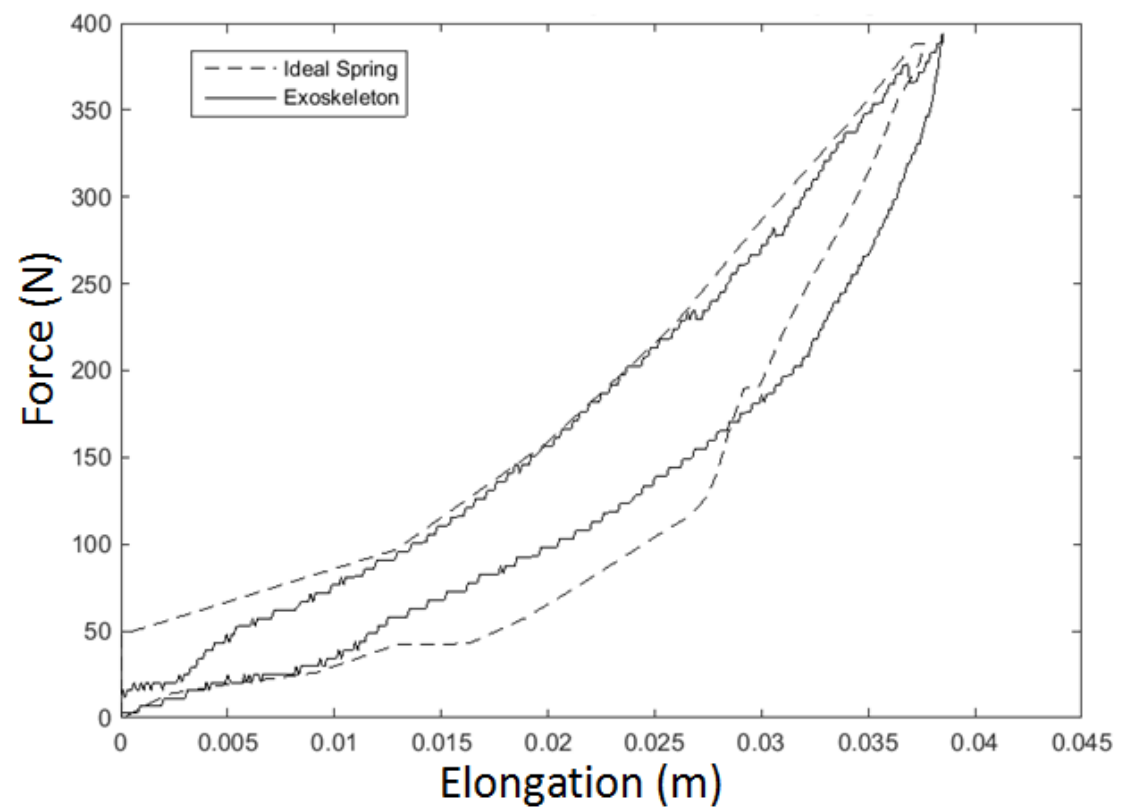

Figure D.6: Mechanical testing results of the ideal spring force vs elongation with the PAM inflated to $55 \mathrm{kPa}$ and with a vertical displacement of $7 \mathrm{~cm}$. The percentage of the ideal spring force was fitted to match the PAM force. The ideal spring force in this case is set to $40 \%$ 


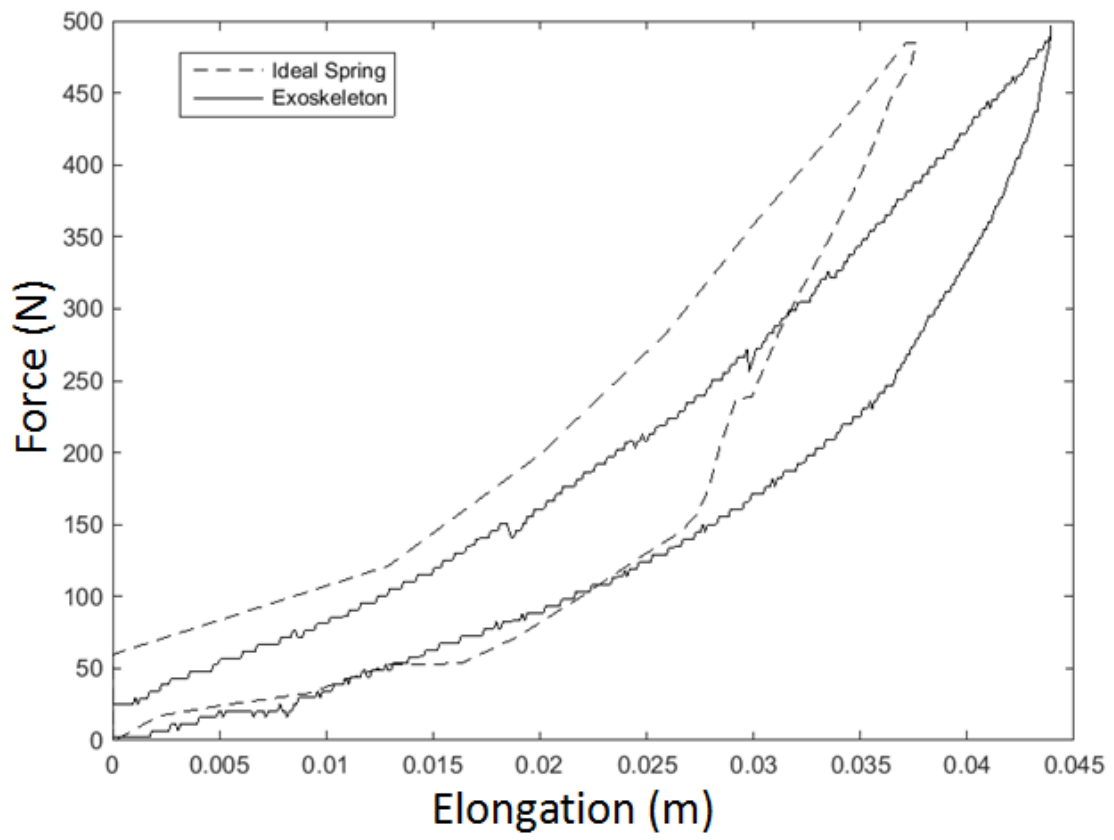

Figure D.7: Mechanical testing results of the ideal spring force vs elongation with the PAM inflated to $48 \mathrm{kPa}$ and with a vertical displacement of $8 \mathrm{~cm}$. The percentage of the ideal spring force was fitted to match the PAM force. The ideal spring force in this case is set to $50 \%$ 


\section{D.3 Comparison of $25 \%$ of biological ankle torque (for $86 \mathrm{~kg}$ indi- vidual) vs torque produced by exoskeleton}

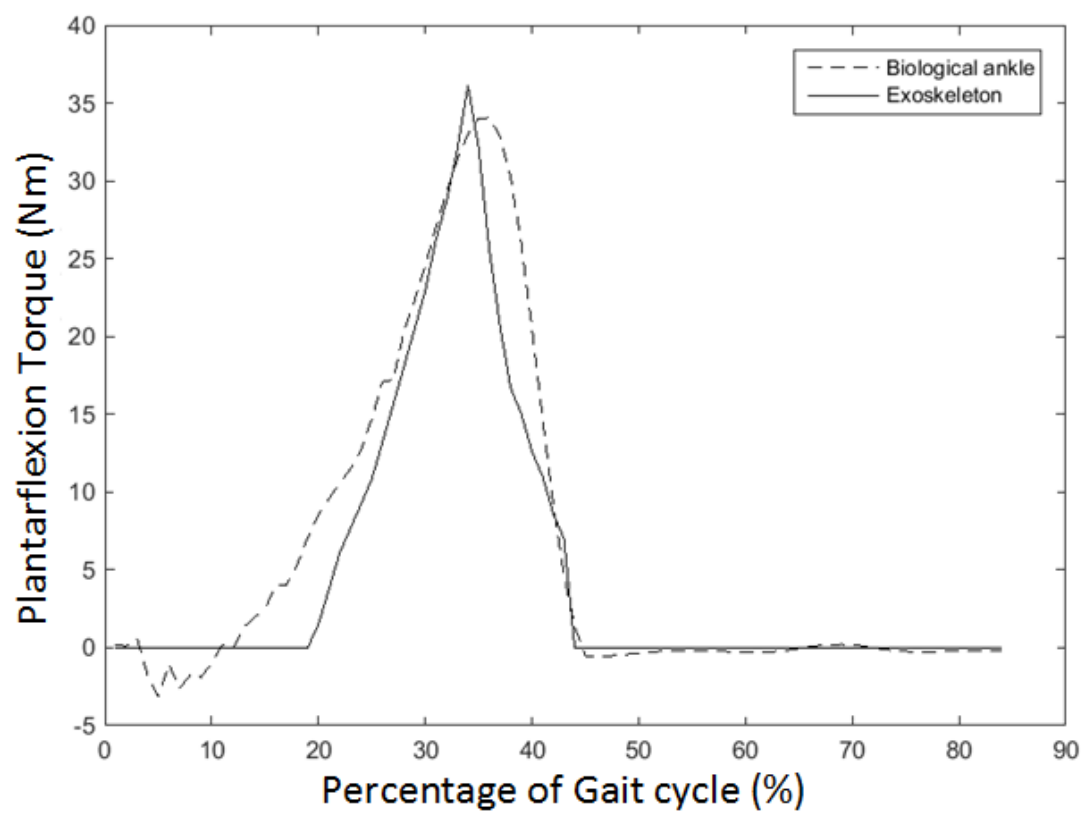

Figure D.8: Mechanical testing results of the torque vs percentage of gait cycle with the PAM inflated to $70 \mathrm{kPa}$ and with a vertical displacement of $5 \mathrm{~cm}$ 


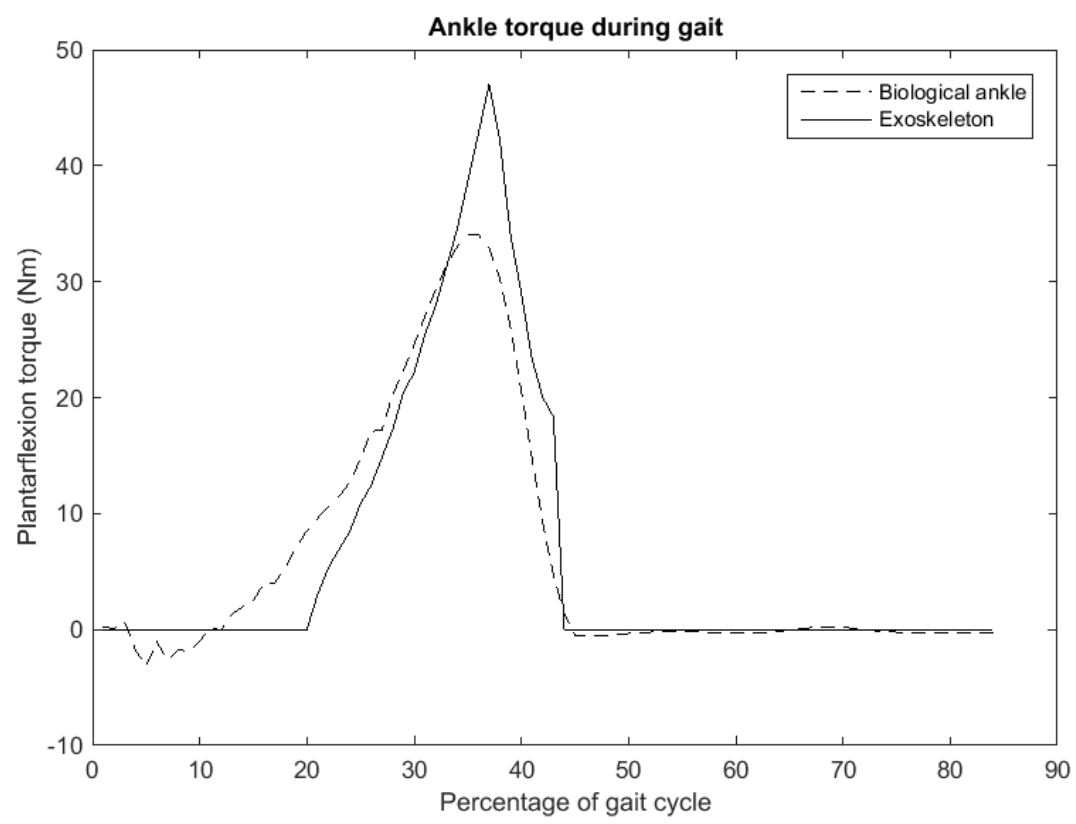

Figure D.9: Mechanical testing results of the torque vs percentage of gait cycle with the PAM inflated to $70 \mathrm{kPa}$ and with a vertical displacement of $6 \mathrm{~cm}$

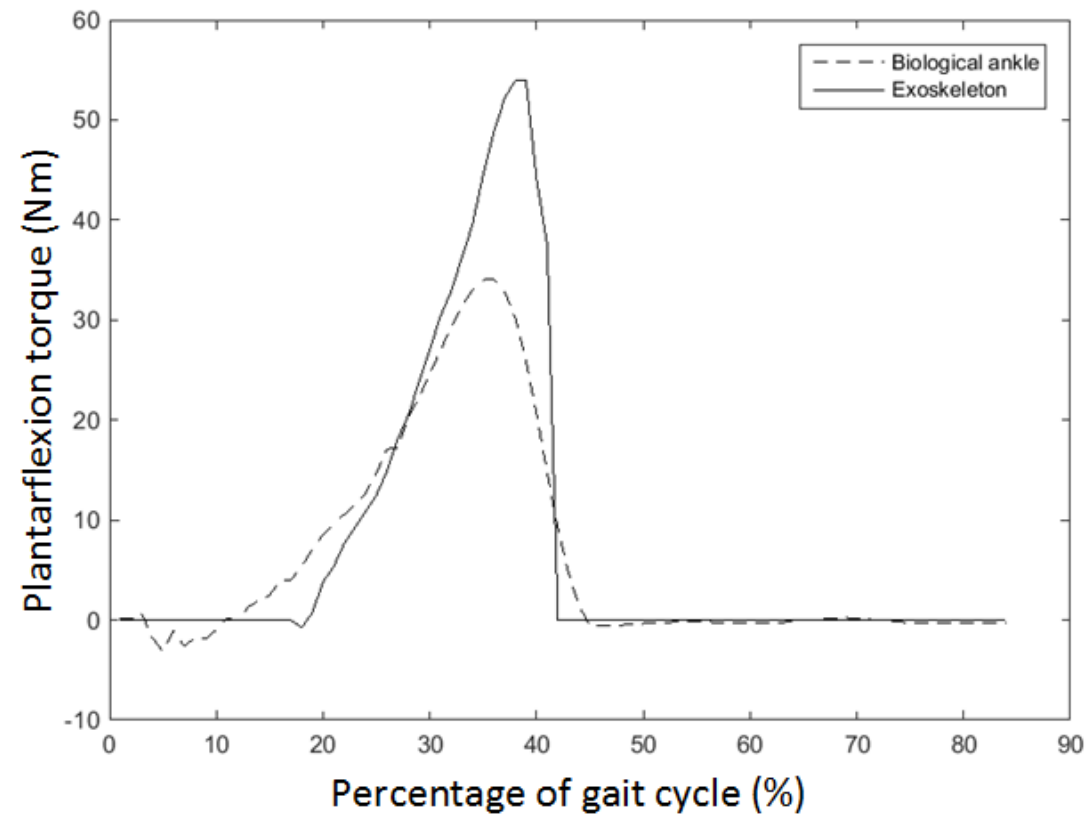

Figure D.10: Mechanical testing results of the torque vs percentage of gait cycle with the PAM inflated to $55 \mathrm{kPa}$ and with a vertical displacement of $7 \mathrm{~cm}$ 


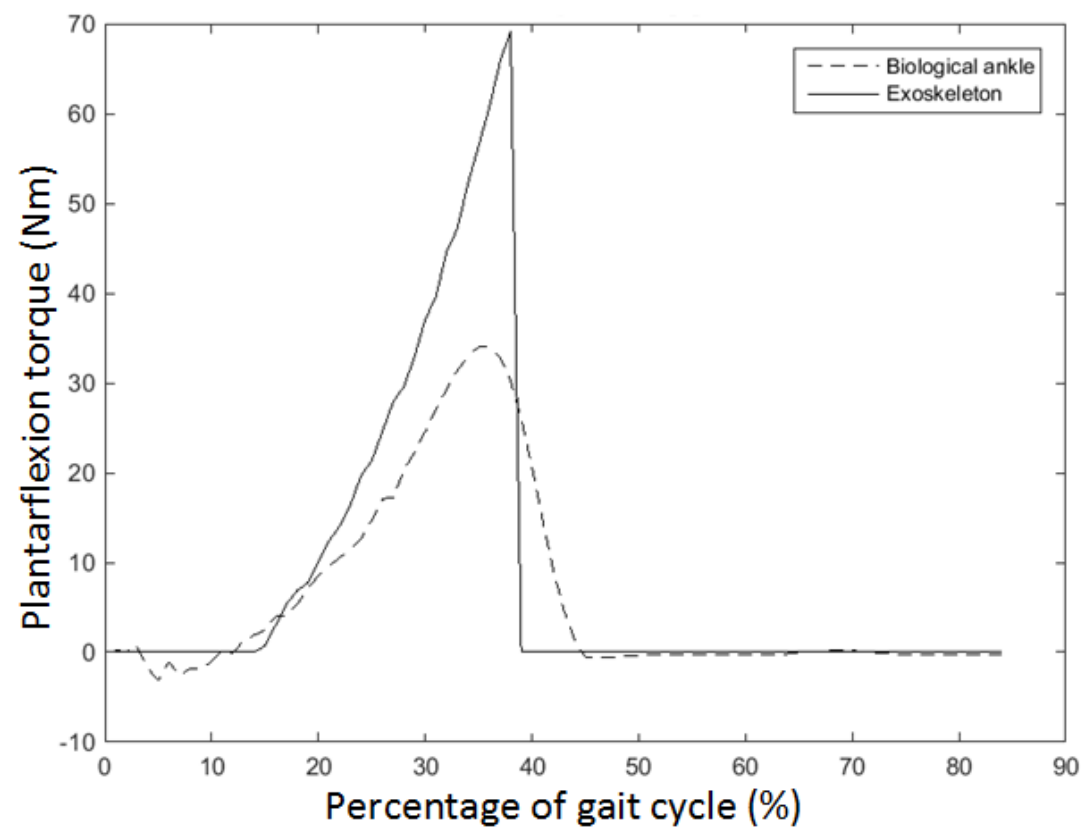

Figure D.11: Mechanical testing results of the torque vs percentage of gait cycle with the PAM inflated to $48 \mathrm{kPa}$ and with a vertical displacement of $8 \mathrm{~cm}$ 
D.4 Comparison of biological ankle torque (for $86 \mathrm{~kg}$ individual) vs torque produced by exoskeleton with percentage of biological ankle torque adjusted to fit PAM with percentage of biological torque adjusted to fit exoskeleton

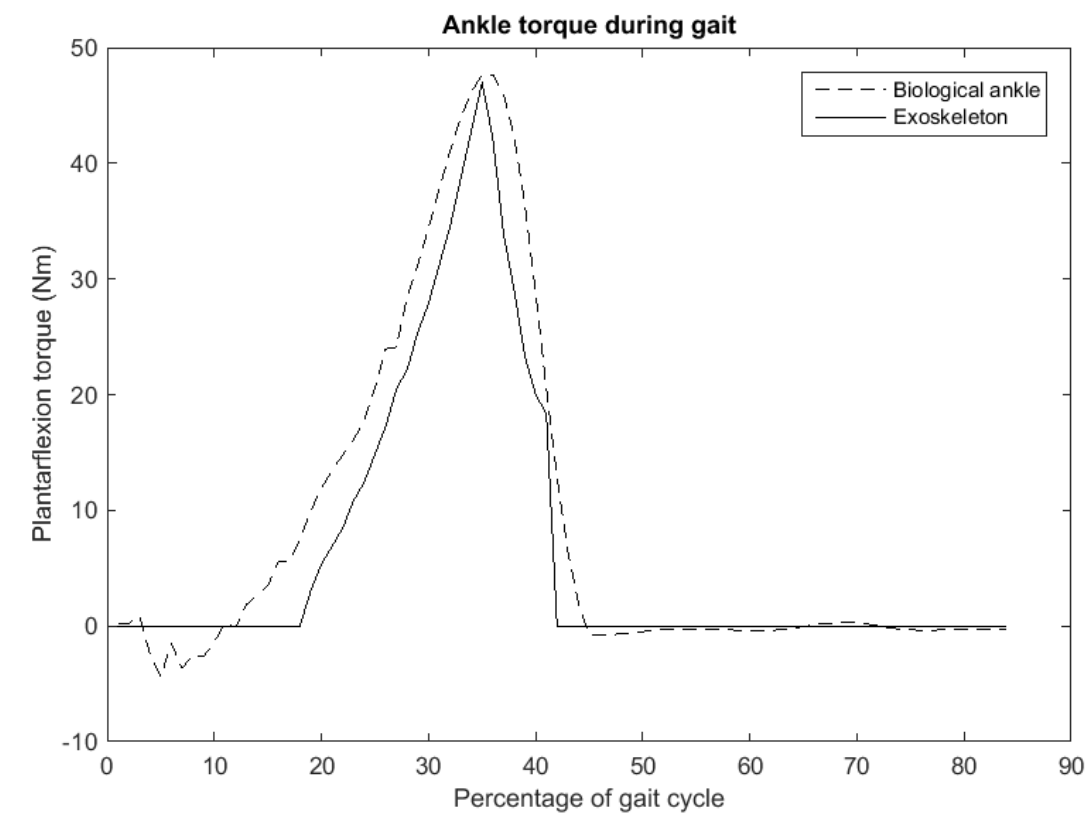

Figure D.12: Mechanical testing results of the torque vs percentage of gait cycle with the PAM inflated to $70 \mathrm{kPa}$ and with a vertical displacement of $6 \mathrm{~cm}$. The percentage of the biological ankle torque was fitted to match the PAM torque. The biological ankle torque in this case is set to $35 \%$ 


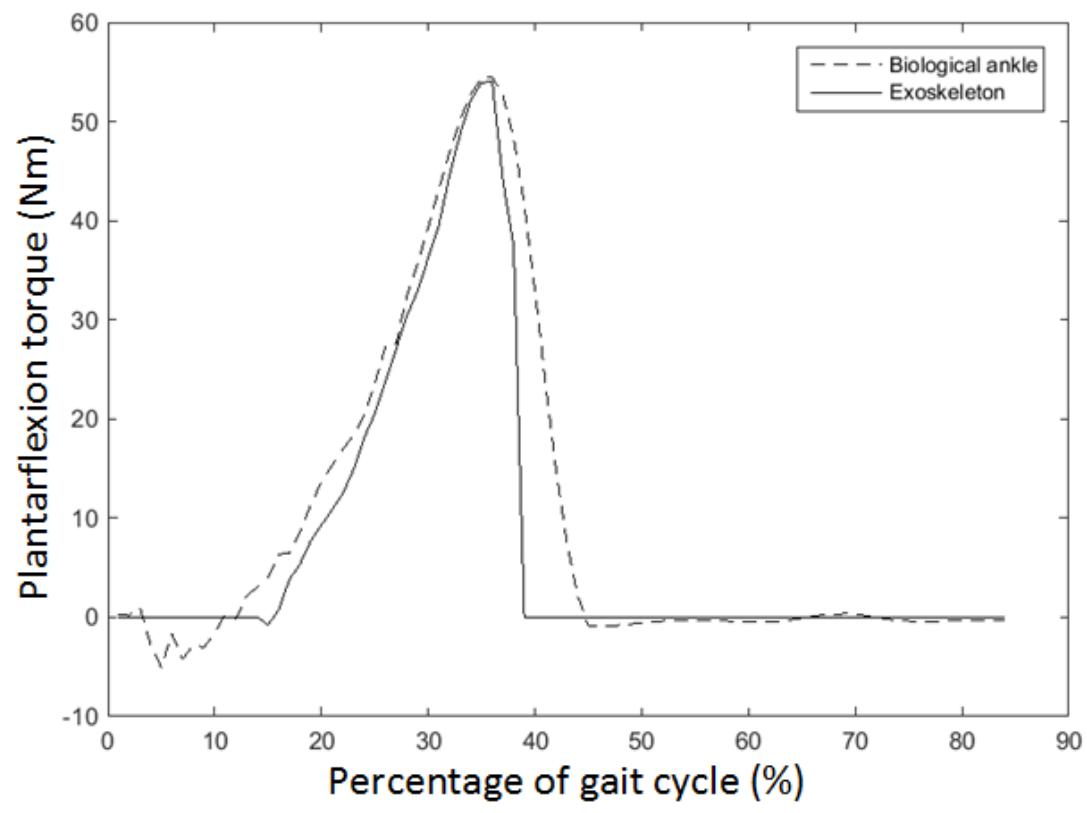

Figure D.13: Mechanical testing results of the torque vs percentage of gait cycle with the PAM inflated to $55 \mathrm{kPa}$ and with a vertical displacement of $7 \mathrm{~cm}$. The percentage of the ideal spring force was fitted to match the PAM force. The ideal spring force in this case is set to $40 \%$ 


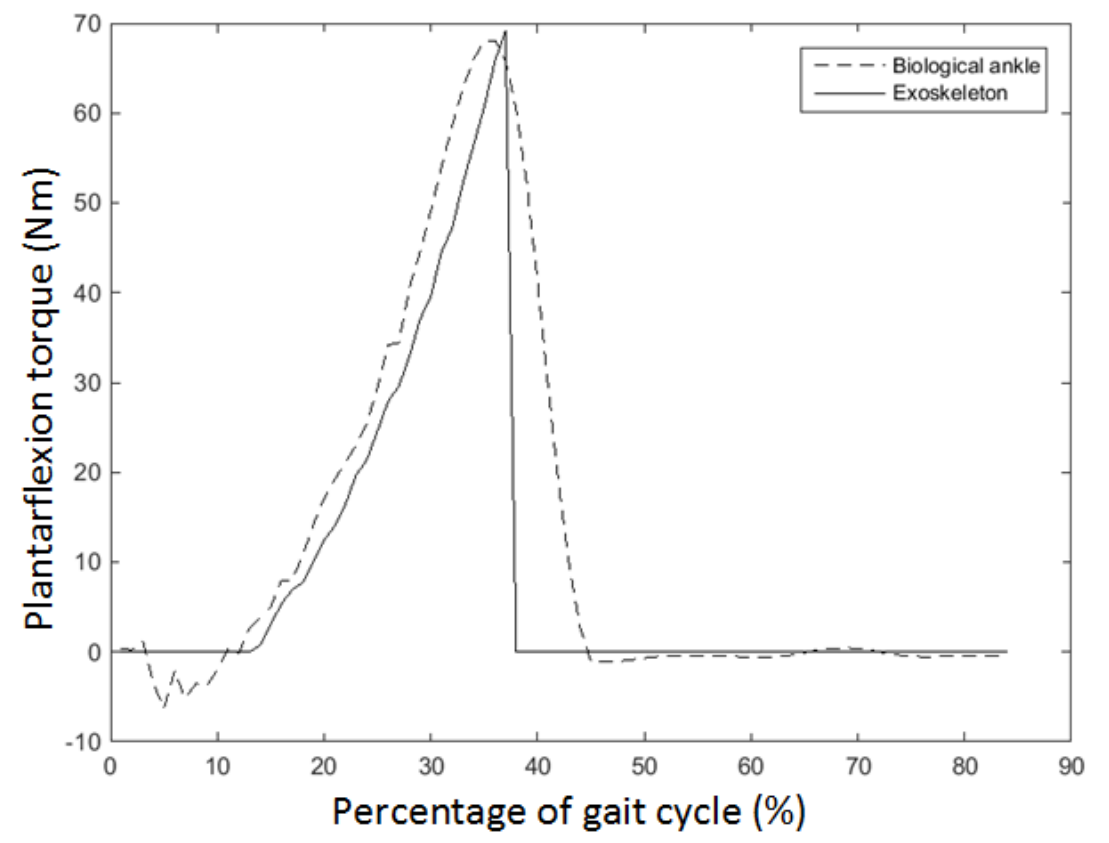

Figure D.14: Mechanical testing results of the torque vs percentage of gait cycle with the PAM inflated to $48 \mathrm{kPa}$ and with a vertical displacement of $8 \mathrm{~cm}$. The percentage of the ideal spring force was fitted to match the PAM force. The ideal spring force in this case is set to $50 \%$ 


\section{Appendix E}

\section{Human Testing Results for participant 1}

\section{E.1 Kinematic results for participant 1}

\section{E.1.1 PAM $70 \mathrm{kPa}$}
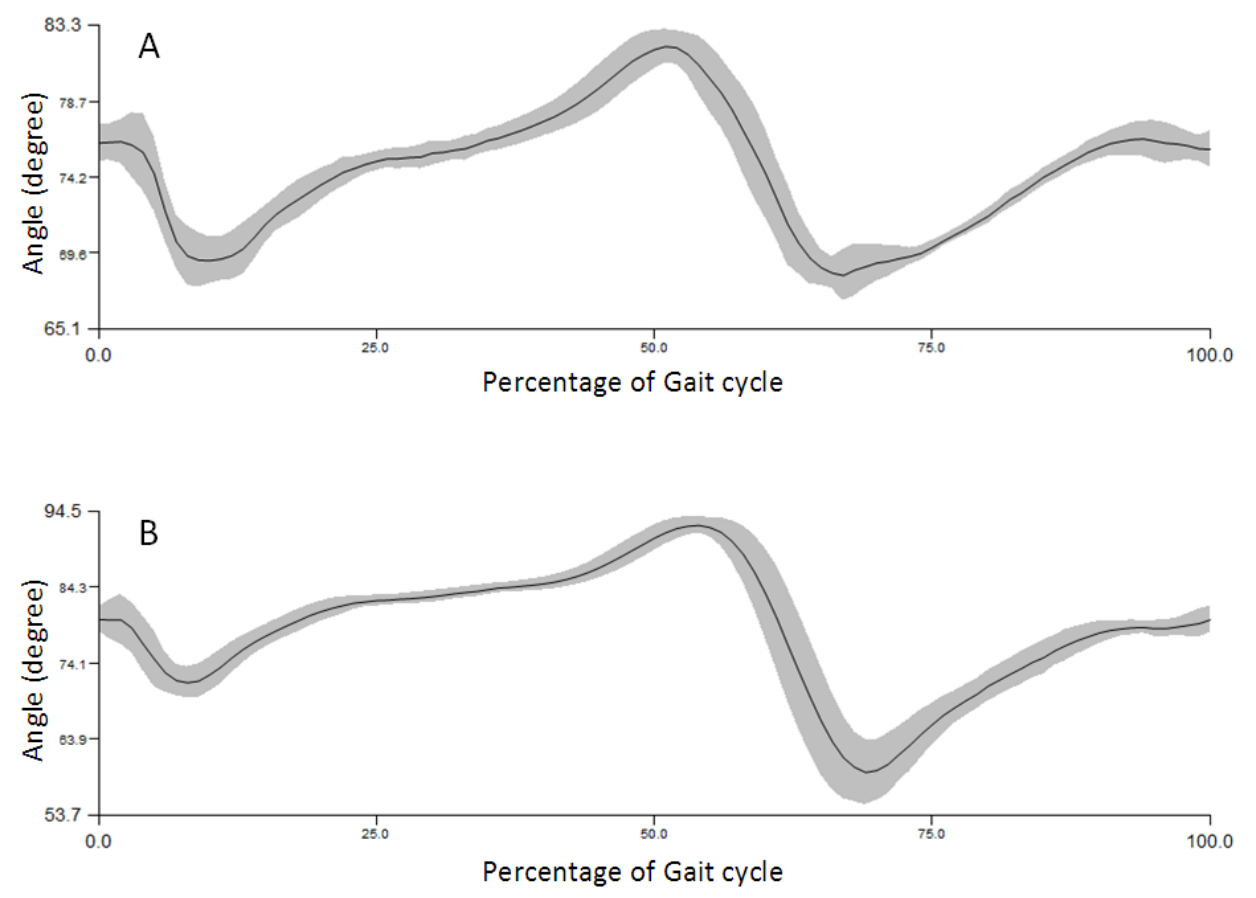

Figure E.1: Walking trial ankle angle for exoskeleton with PAM inflated to $70 \mathrm{kPa}$ 

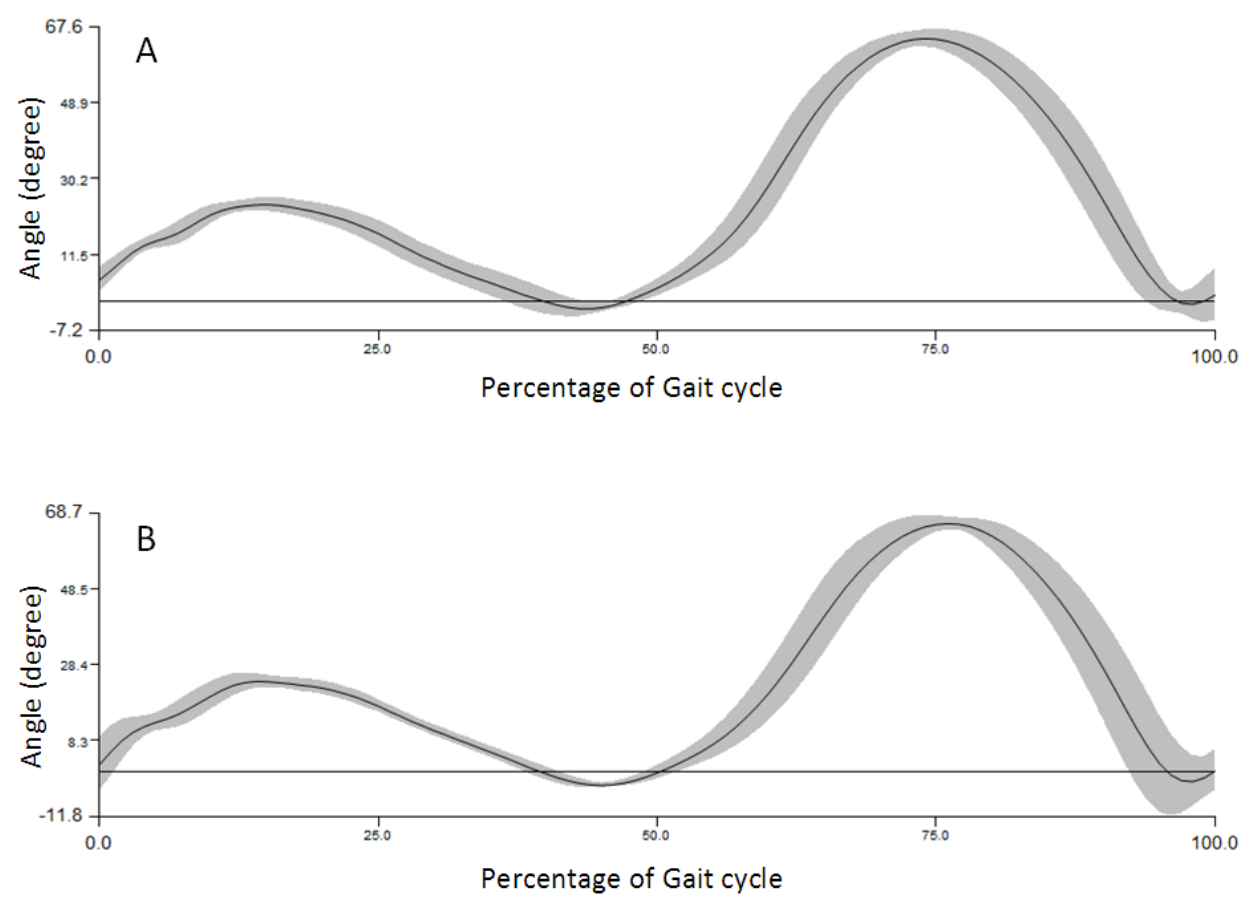

Figure E.2: Walking trial knee angle for exoskeleton with PAM inflated to $70 \mathrm{kPa}$ 

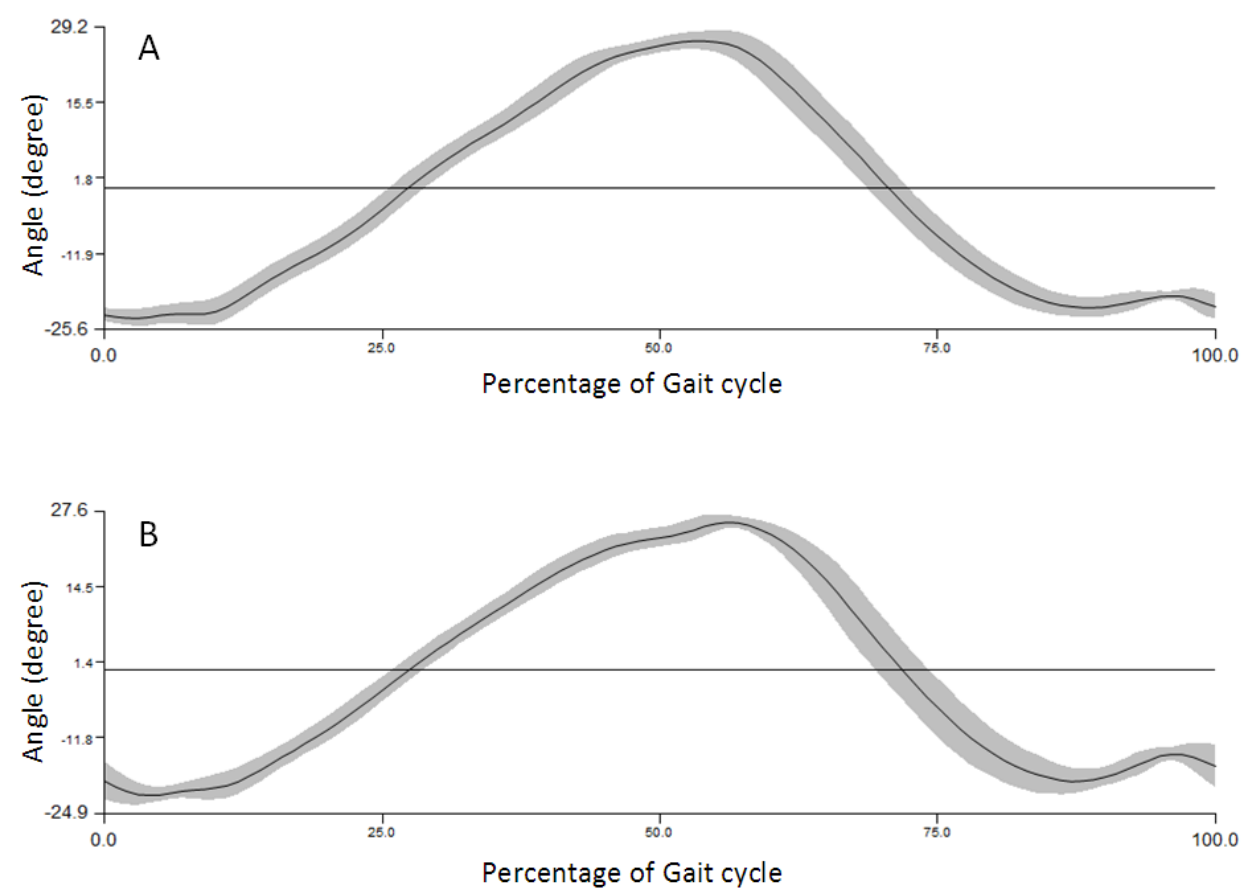

Figure E.3: Walking trial hip angle for exoskeleton with PAM inflated to $70 \mathrm{kPa}$

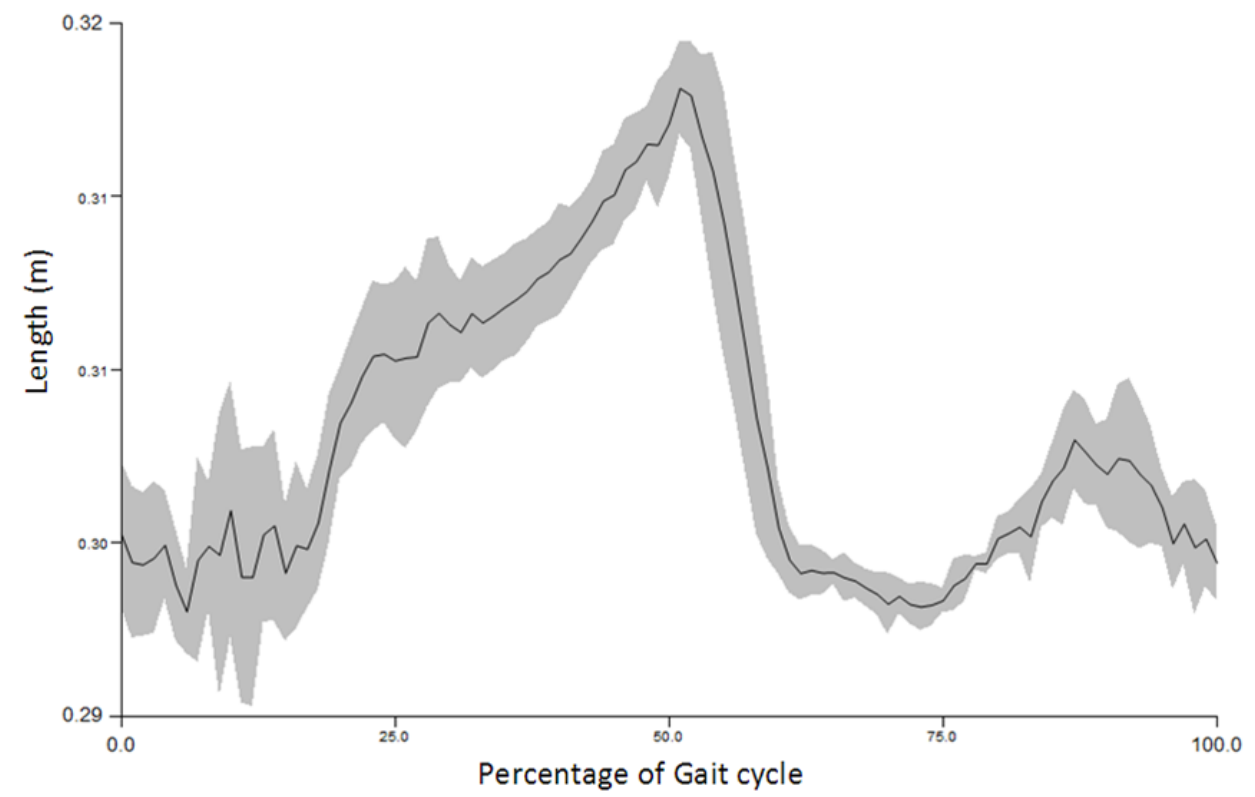

Figure E.4: Walking trial spring length for exoskeleton with PAM inflated to $70 \mathrm{kPa}$ 


\section{E.1.2 PAM $40 \mathrm{kPa}$}
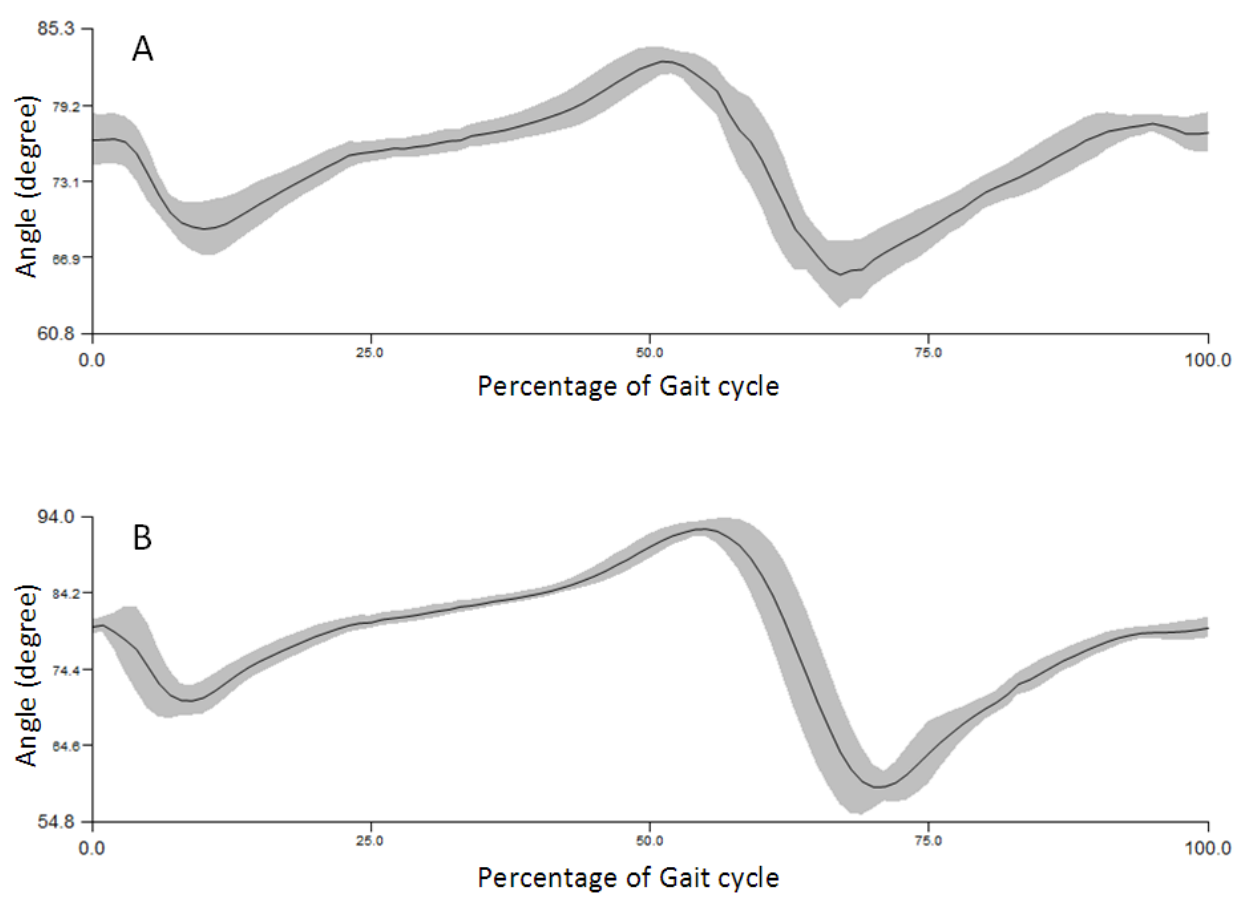

Figure E.5: Walking trial ankle angle for exoskeleton with PAM inflated to $40 \mathrm{kPa}$ 

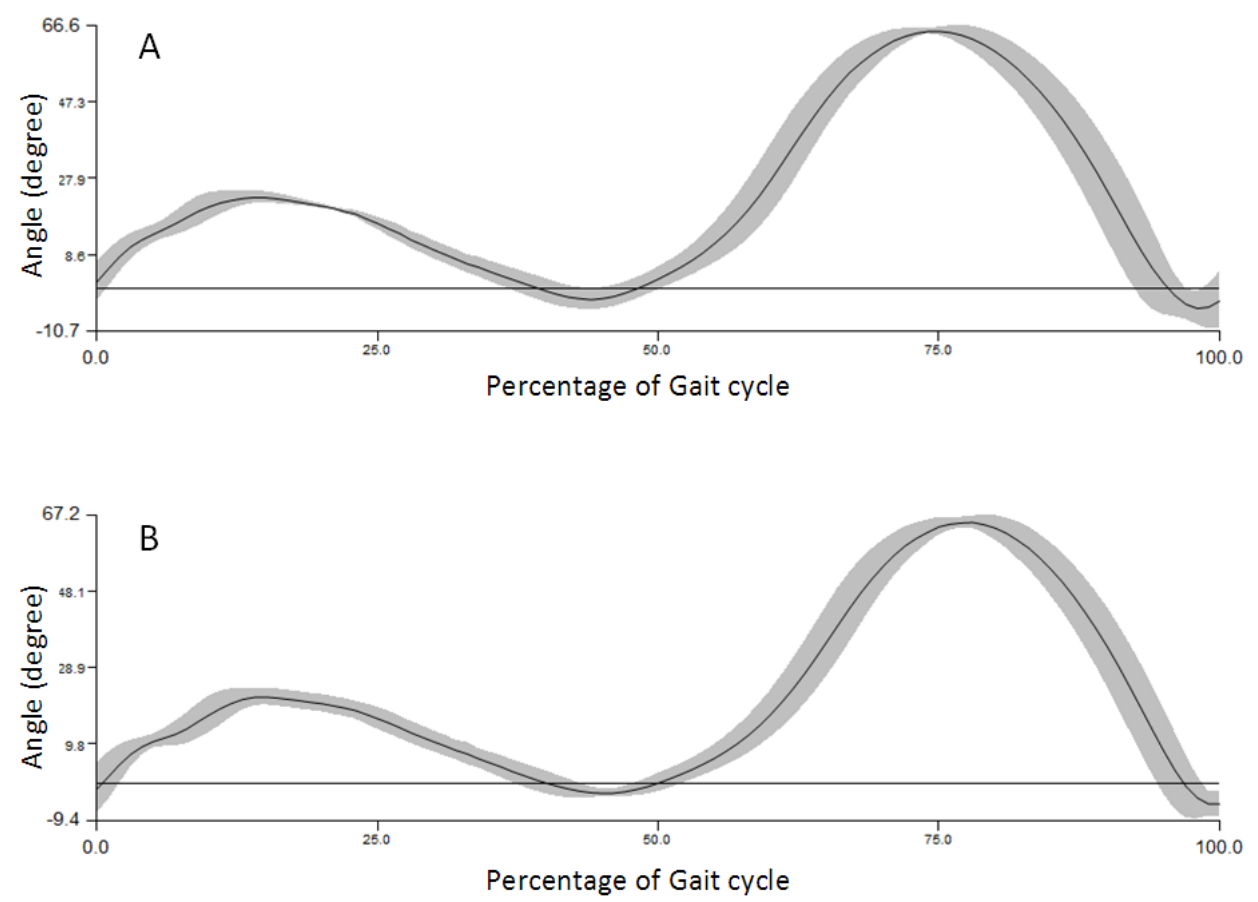

Figure E.6: Walking trial knee angle for exoskeleton with PAM inflated to $40 \mathrm{kPa}$ 

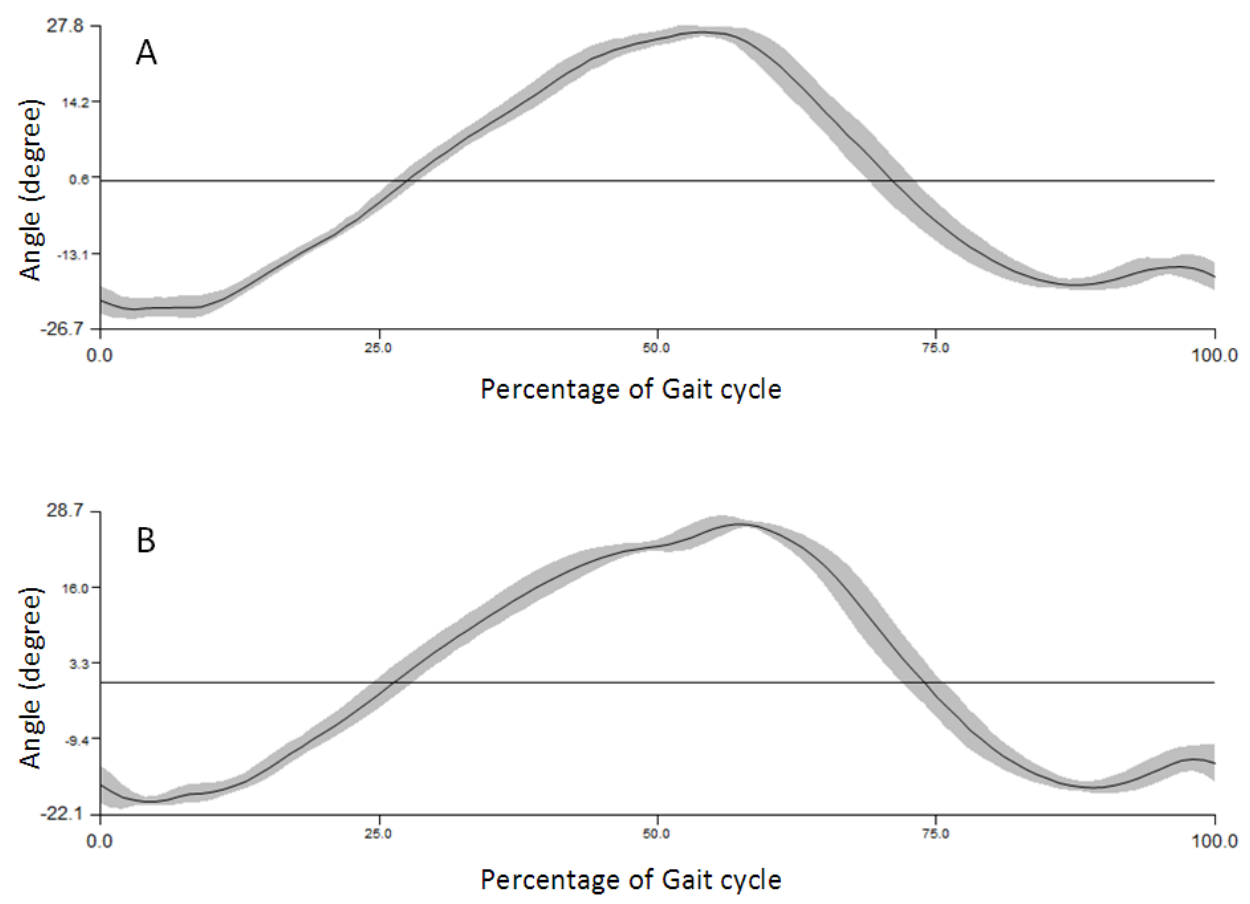

Figure E.7: Walking trial hip angle for exoskeleton with PAM inflated to $40 \mathrm{kPa}$

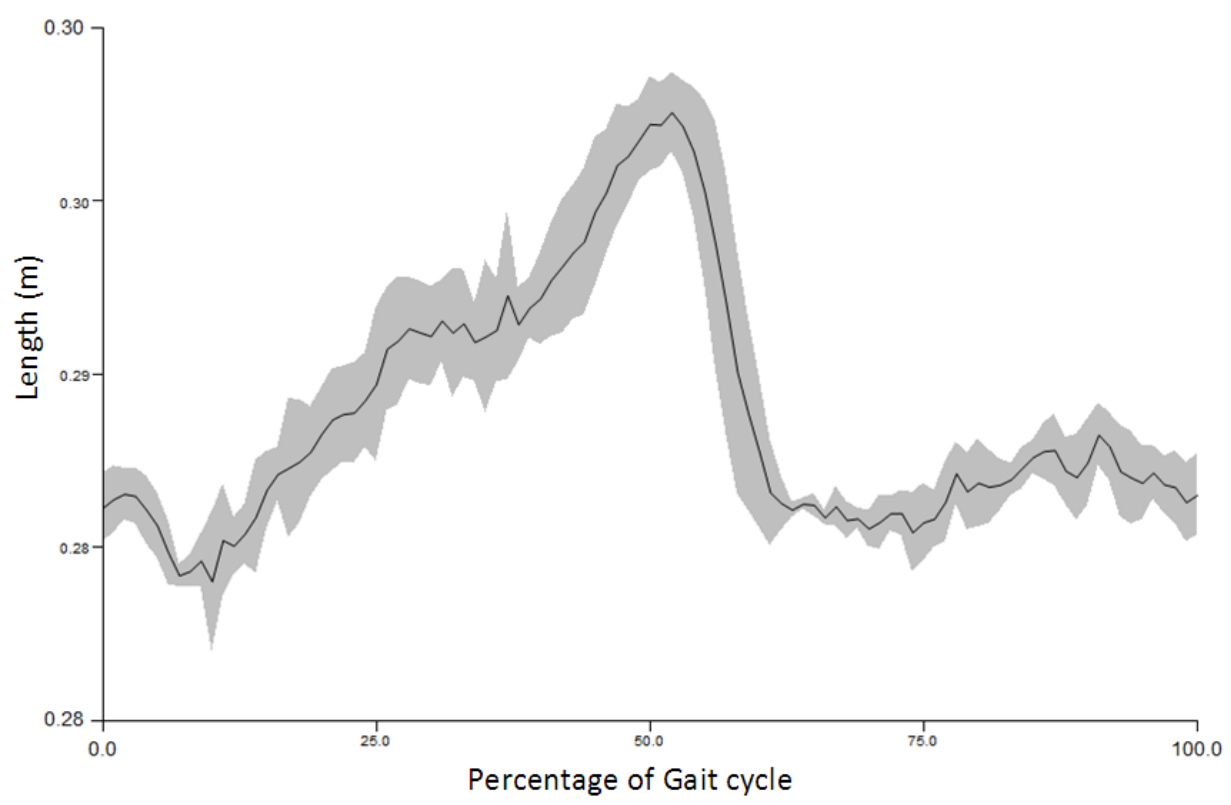

Figure E.8: Walking trial spring length for exoskeleton with PAM inflated to $40 \mathrm{kPa}$ 


\section{E.1.3 Elastic Spring Element}
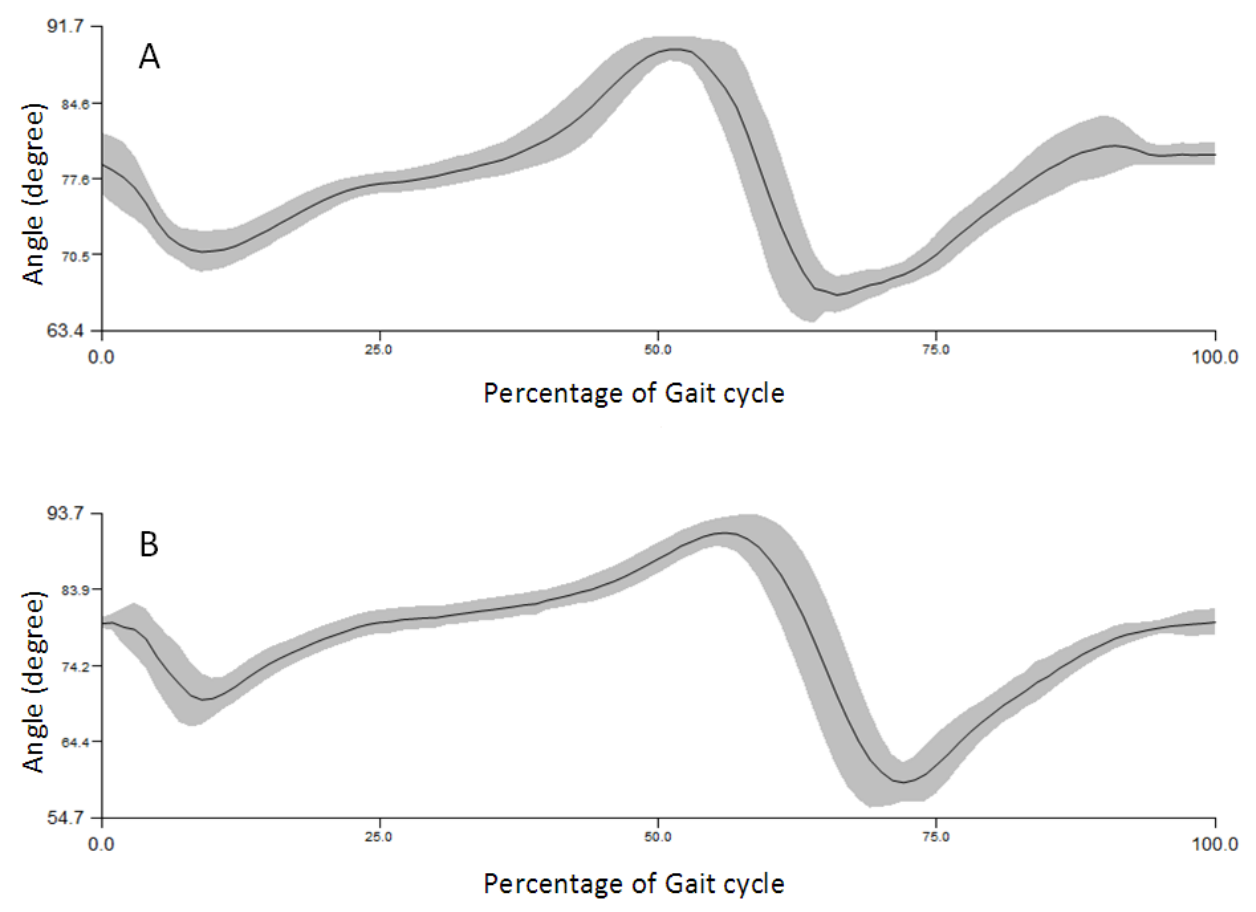

Figure E.9: Walking trial ankle angle for exoskeleton with rubber band used as spring element 

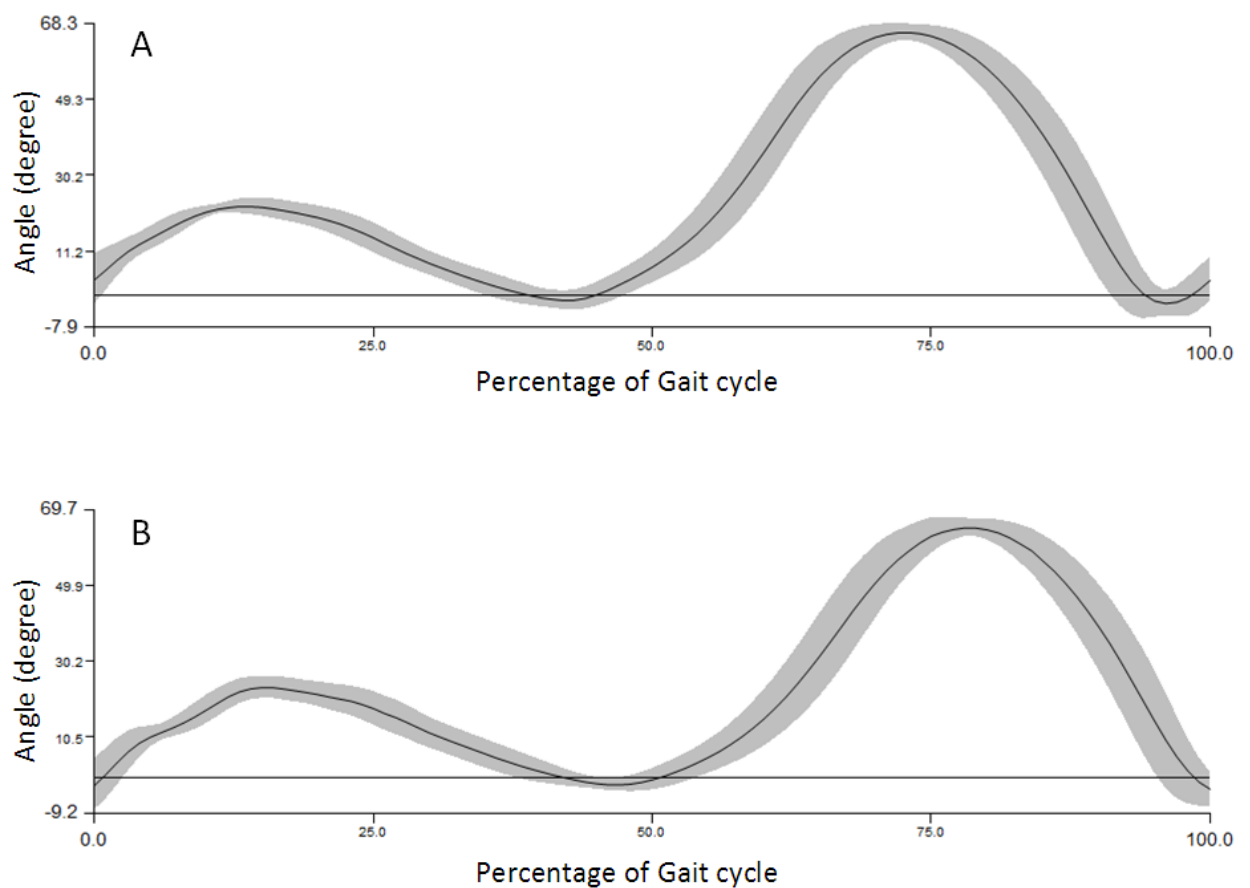

Figure E.10: Walking trial knee angle for exoskeleton with rubber band used as spring element 

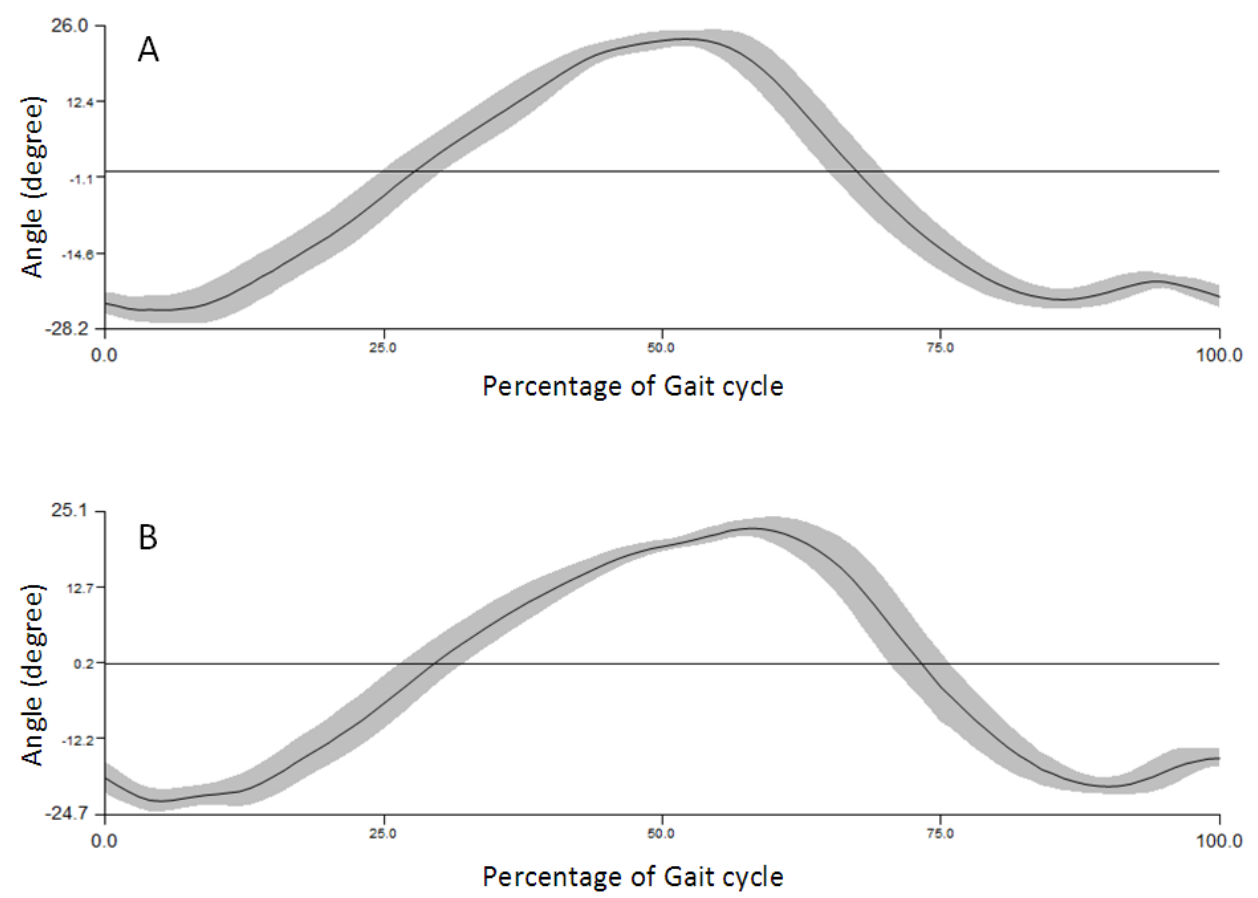

Figure E.11: Walking trial hip angle for exoskeleton with rubber band used as spring element

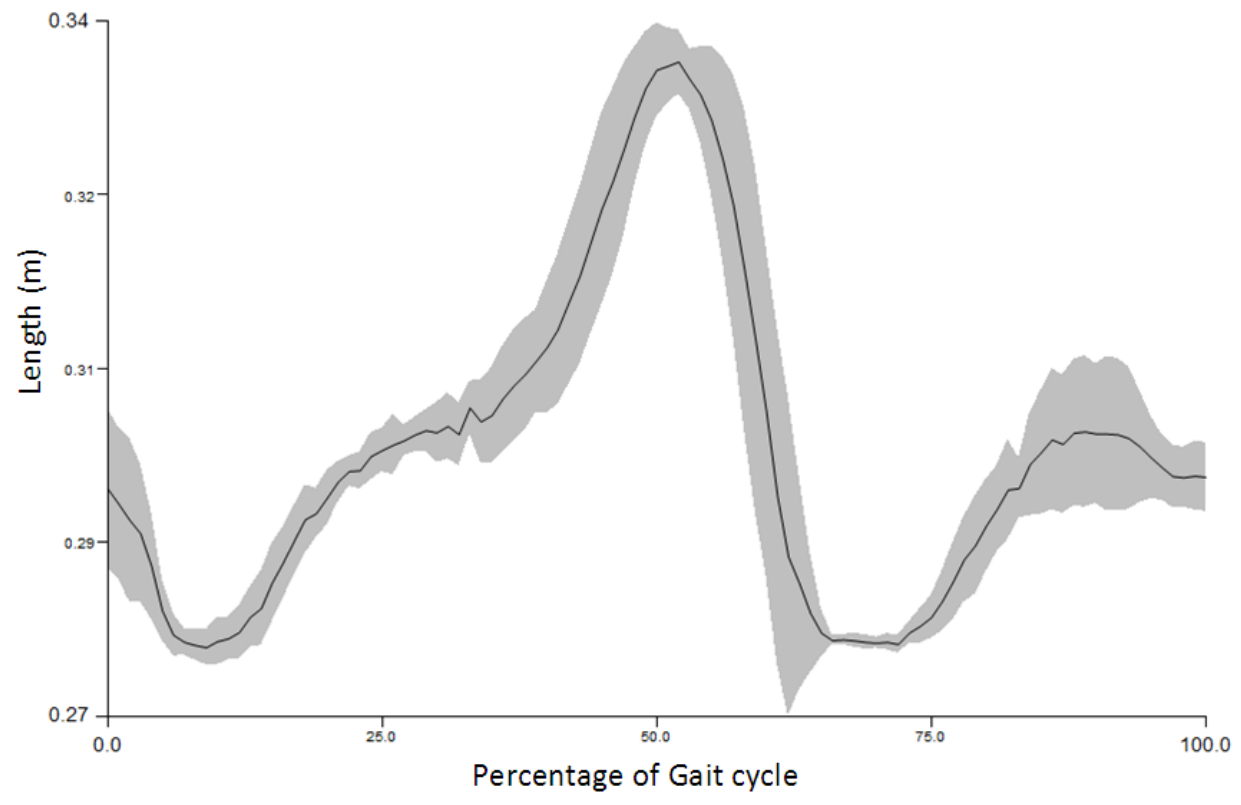

Figure E.12: Walking trial spring length for exoskeleton with rubber band used as spring element 


\section{E.1.4 No Spring Element}
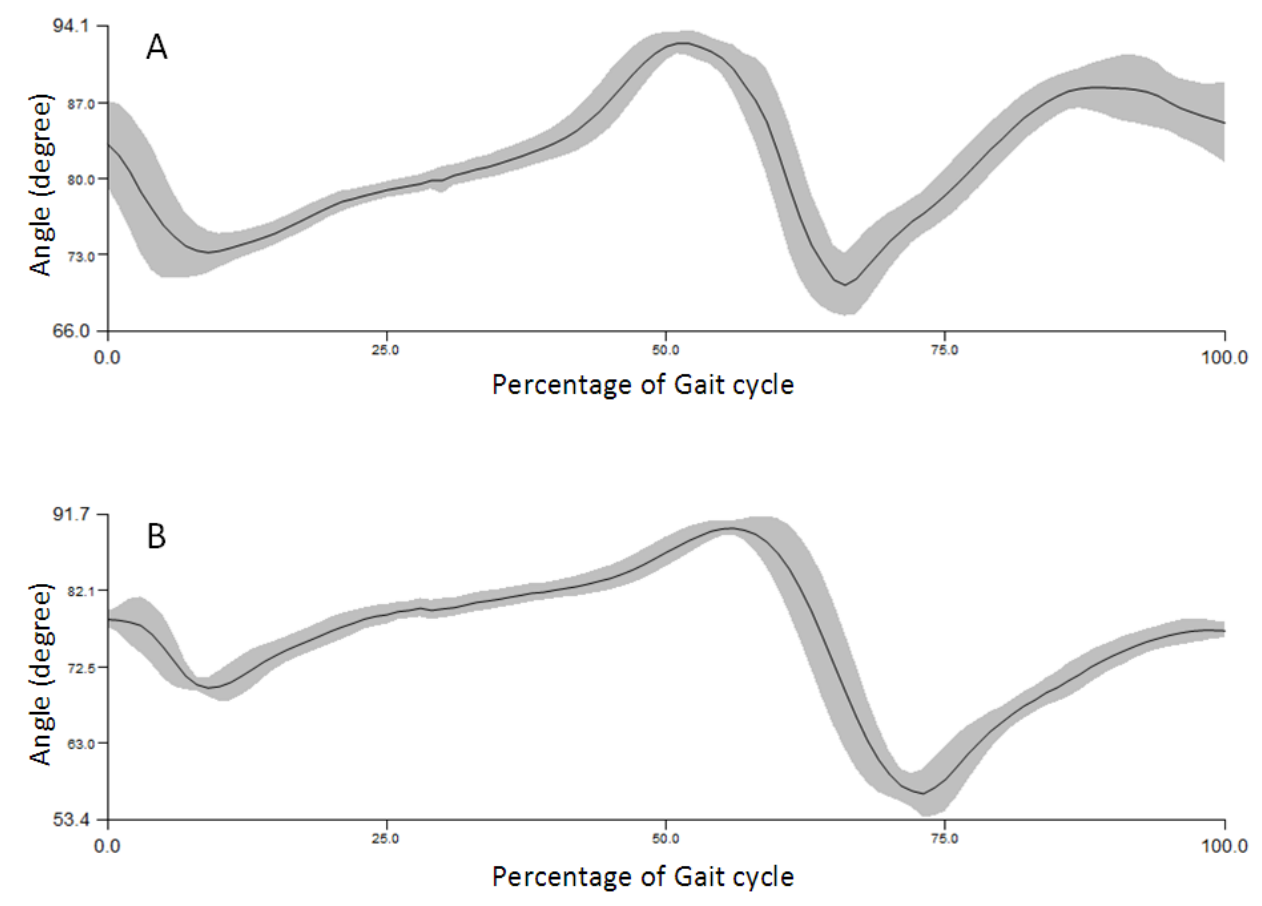

Figure E.13: Walking trial ankle angle for exoskeleton with no spring element 

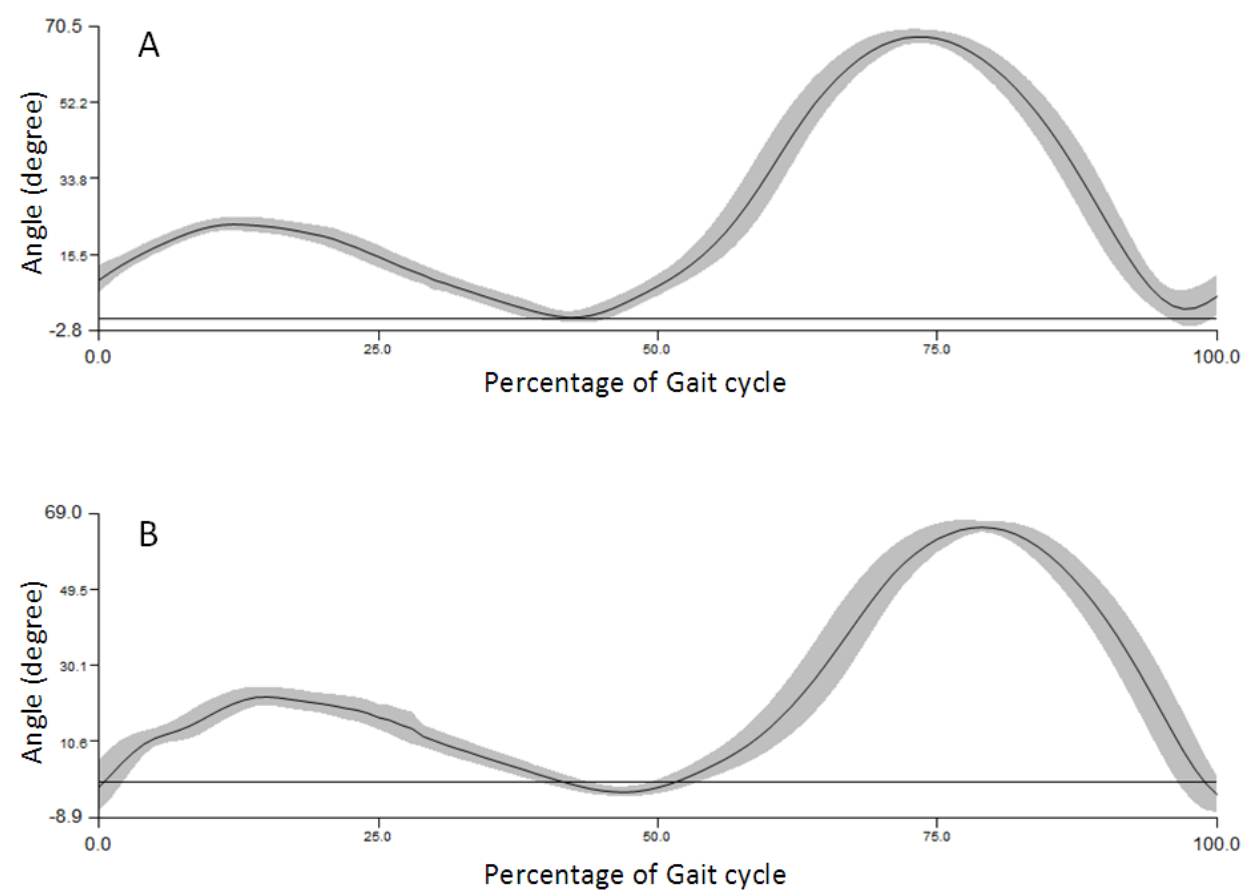

Figure E.14: Walking trial knee angle for exoskeleton with no spring element 

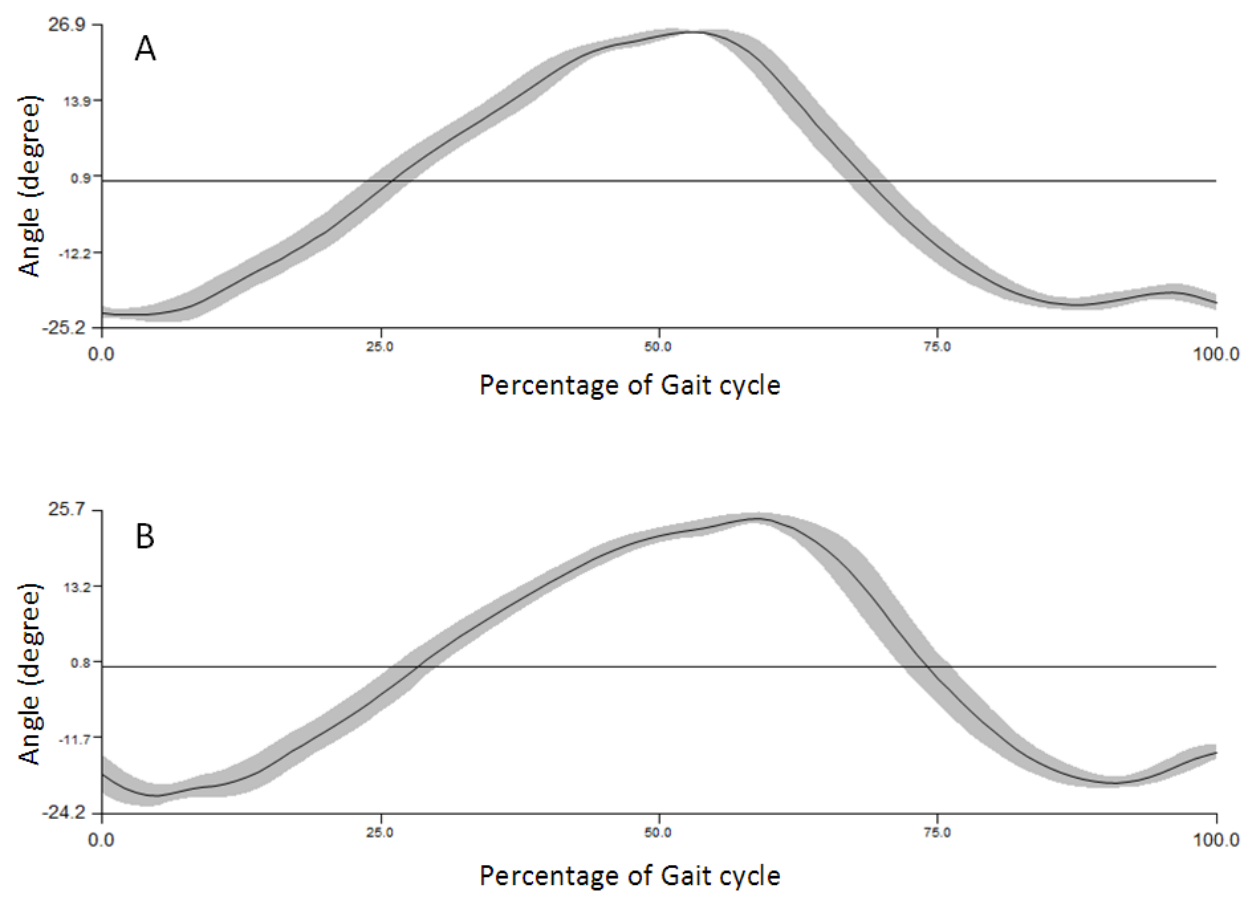

Figure E.15: Walking trial hip angle for exoskeleton with no spring element

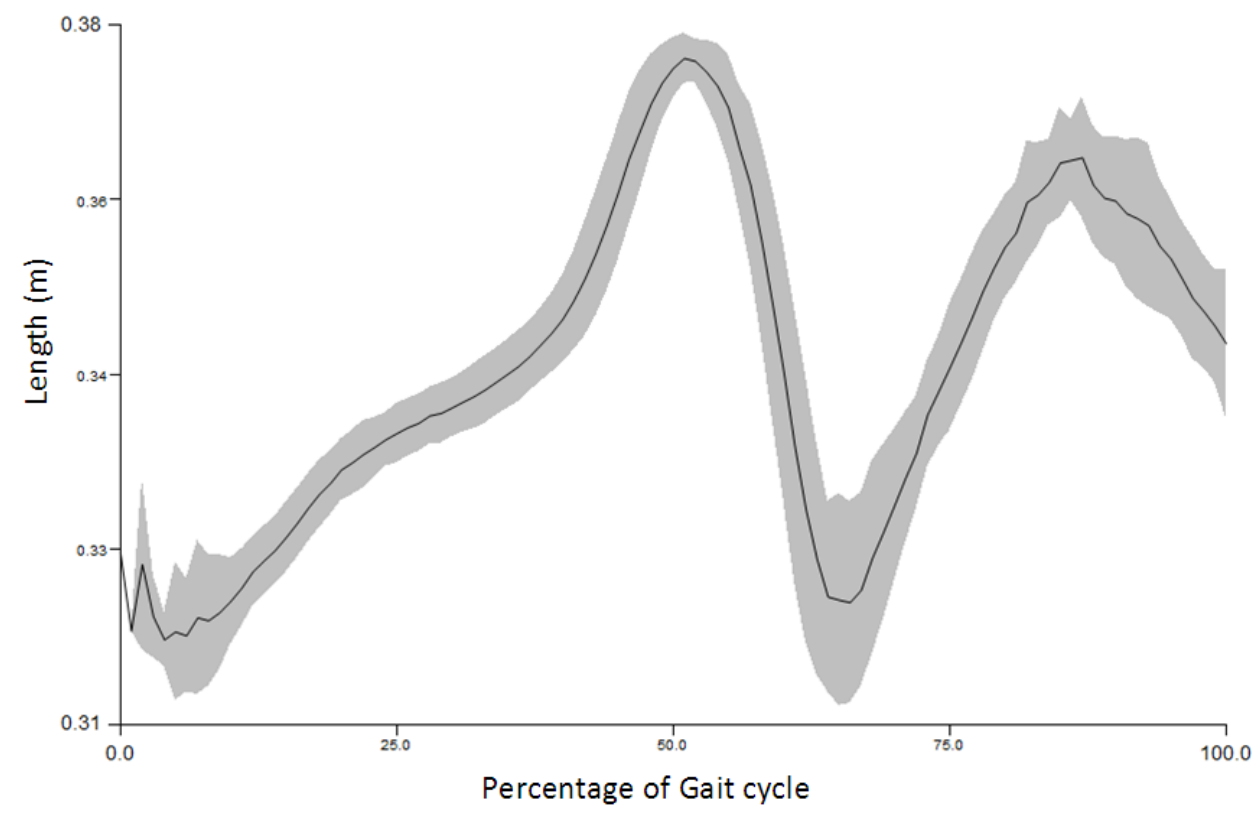

Figure E.16: Walking trial spring length for exoskeleton no spring element. In this case it is the length between the anchor points 


\section{E.1.5 Baseline Walking (No Exo)}
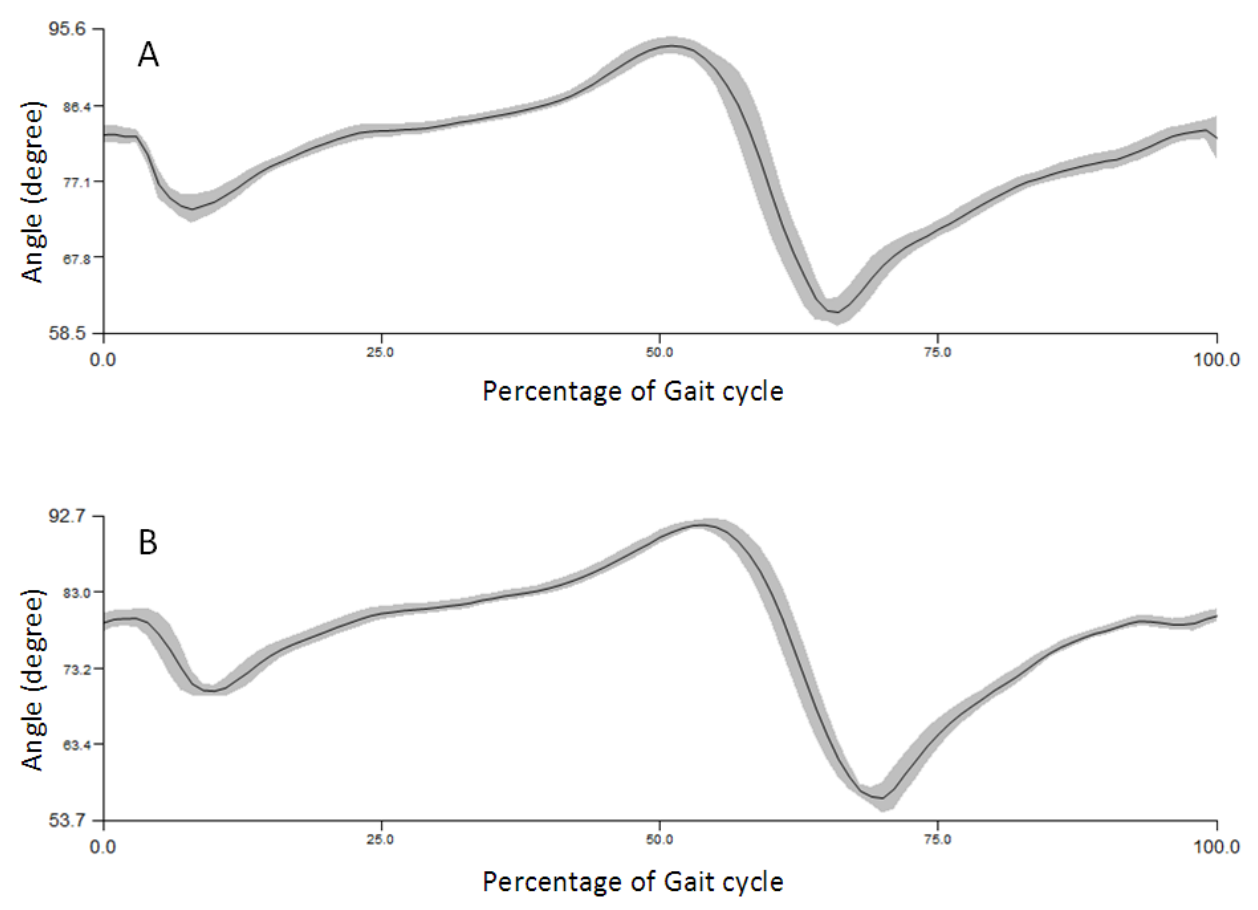

Figure E.17: Ankle angle for baseline walking trial (without exoskeleton) 

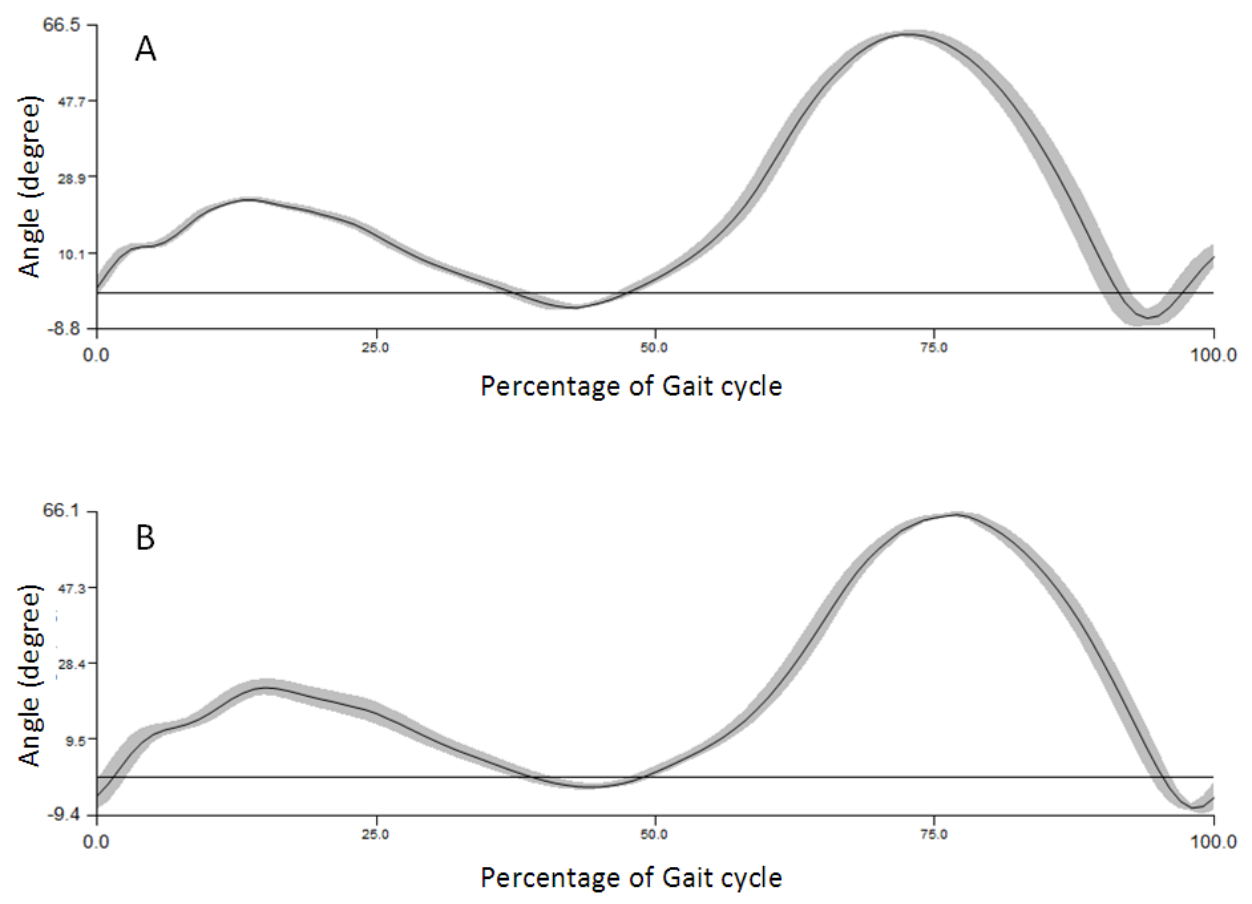

Figure E.18: Knee angle for baseline walking trial (without exoskeleton) 

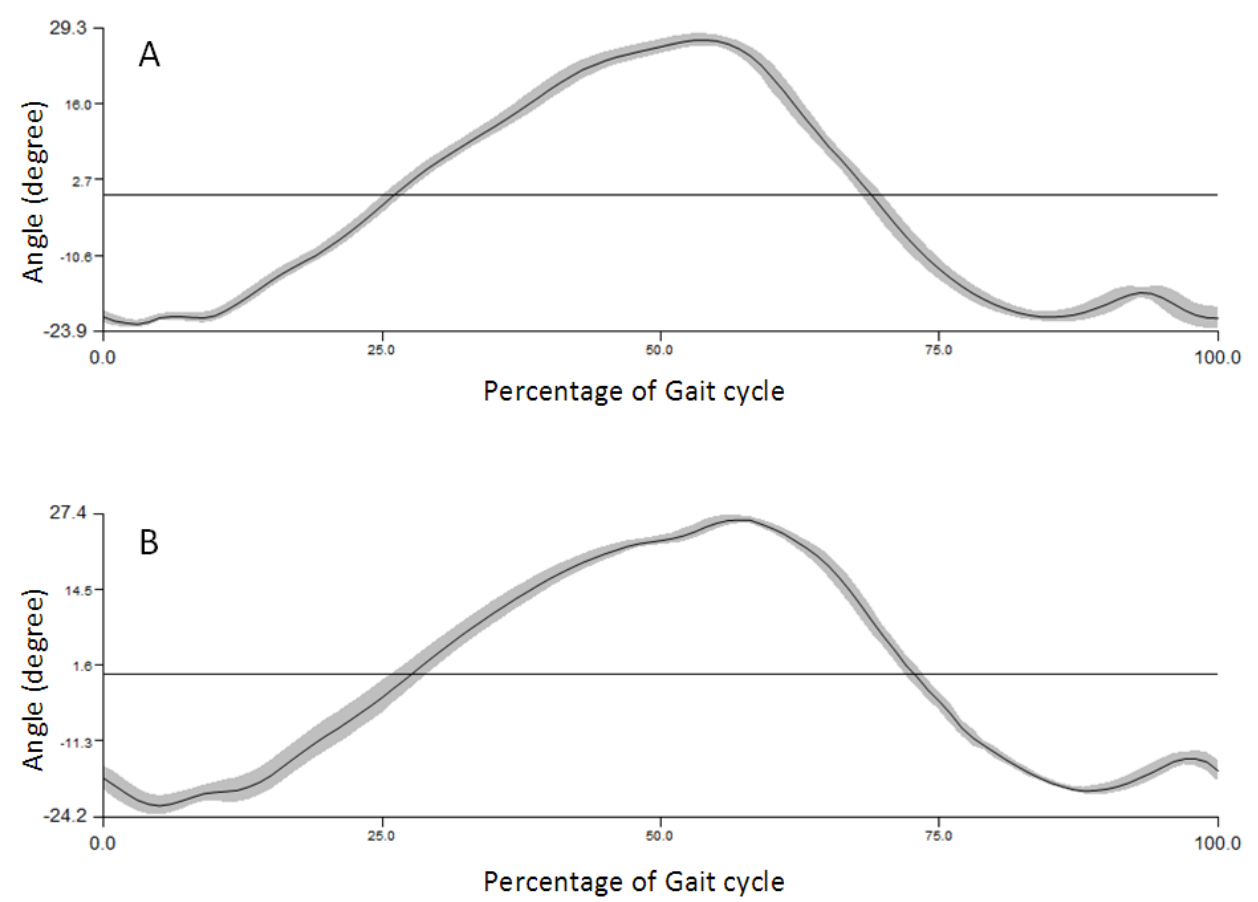

Figure E.19: Hip angle for baseline walking trial (without exoskeleton) 


\section{E.2 Participant 1 EMG walking trials results}
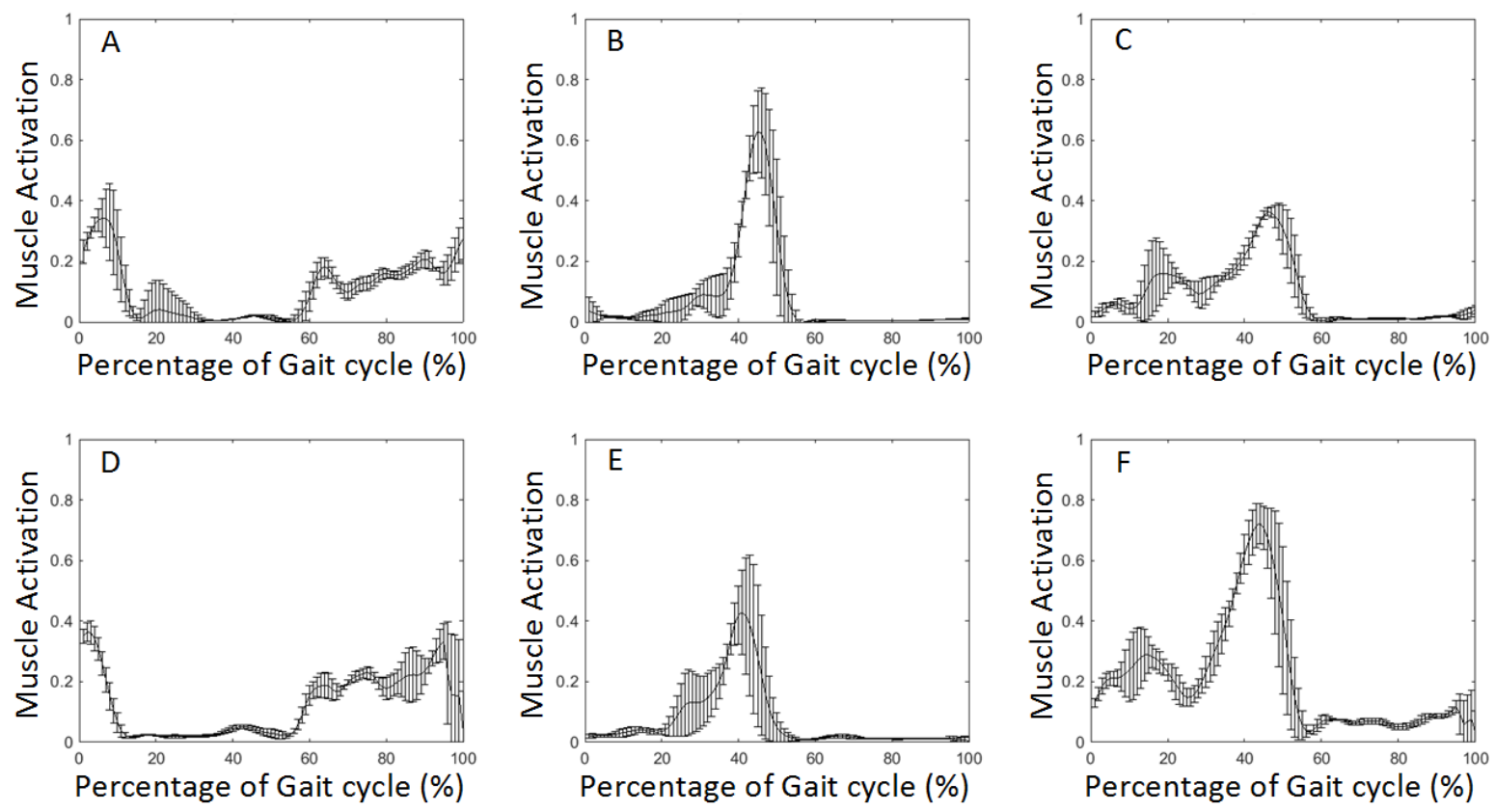

Figure E.20: EMG output of the walking trial without exoskeleton. For participant 1
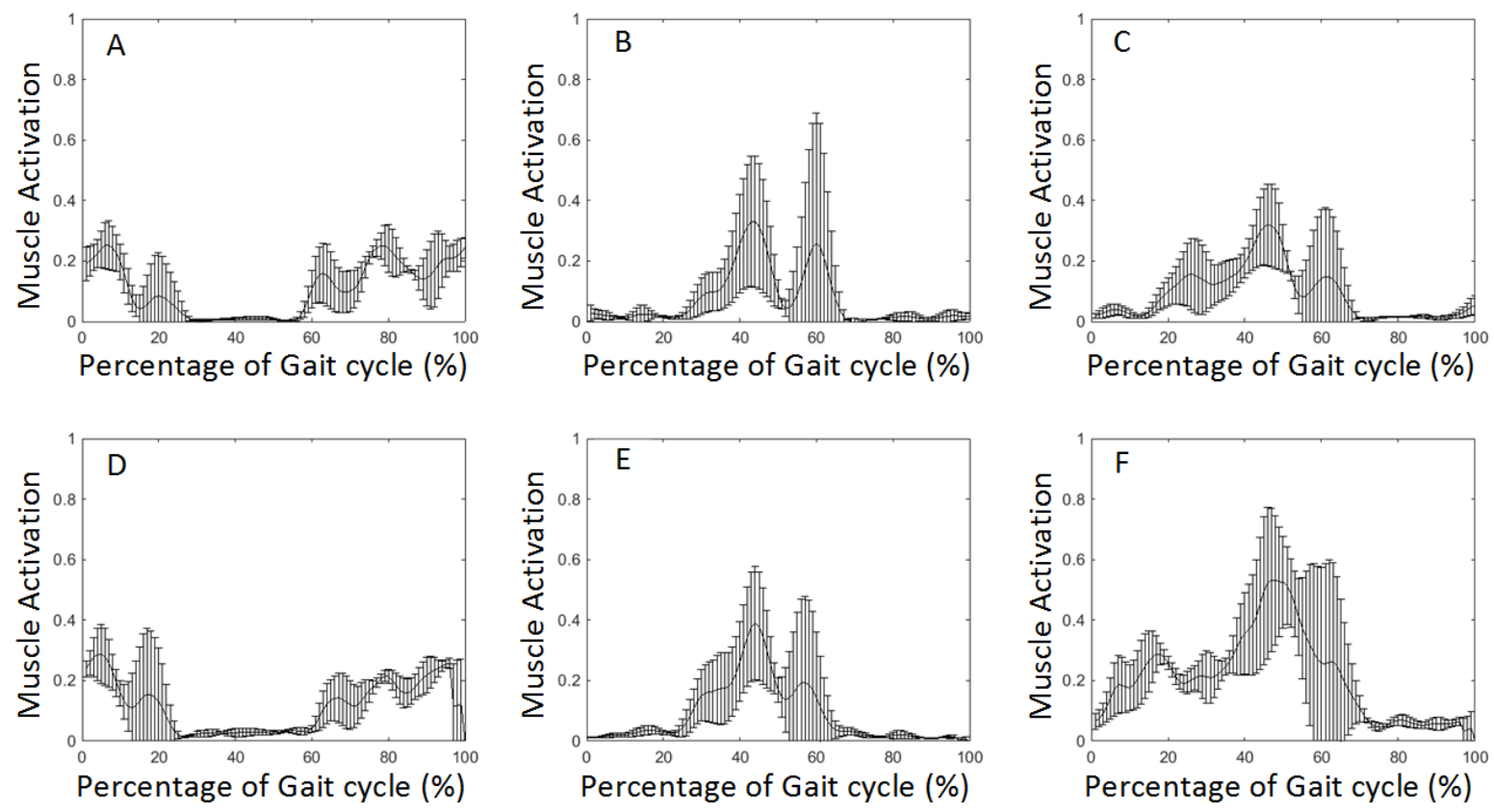

Figure E.21: EMG output of the walking trial with the exoskeleton but with no spring element. For participant 1 

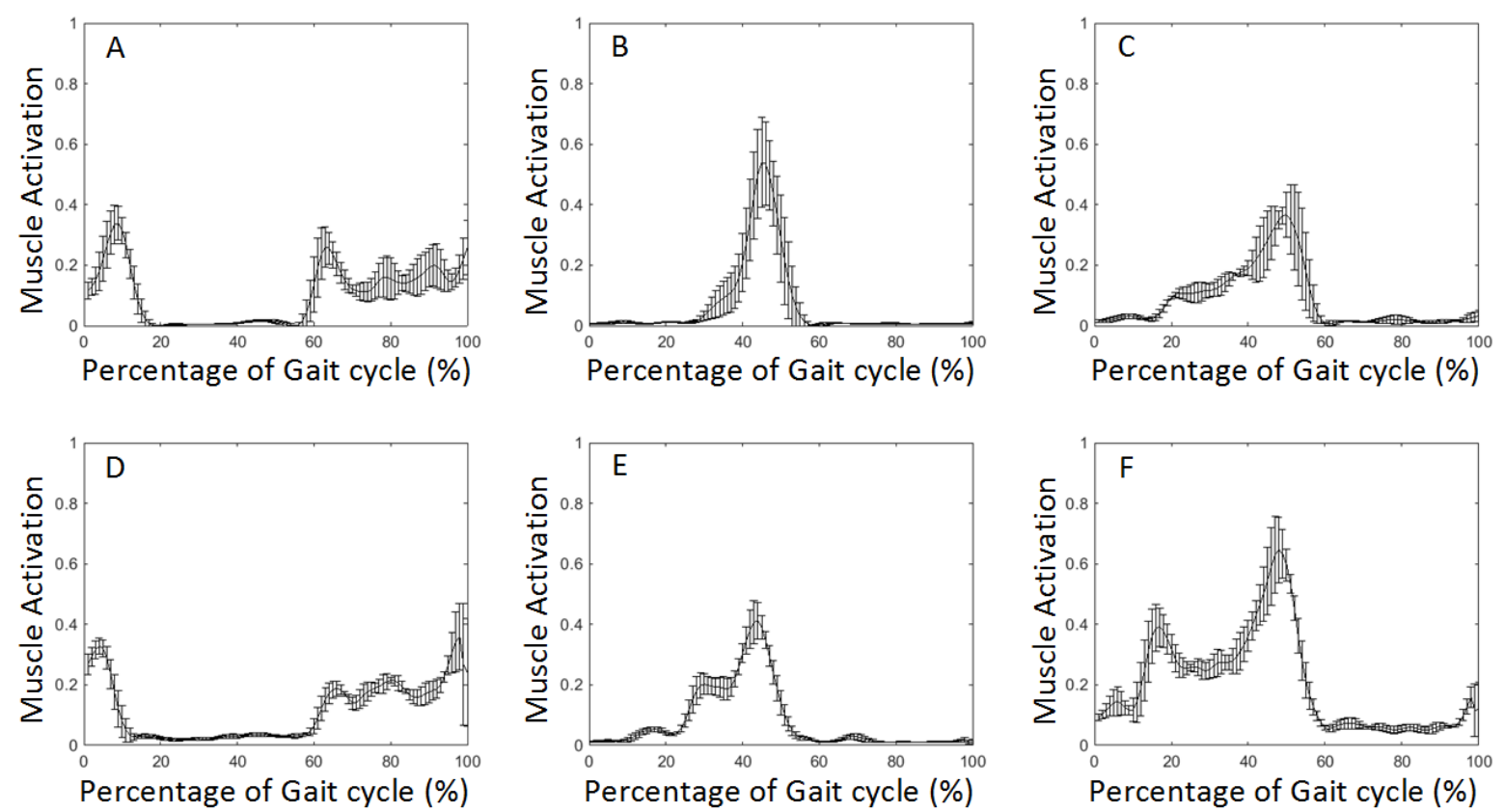

Figure E.22: EMG output of the walking trial with the exoskeleton with an elastic band as the spring element. For participant 1
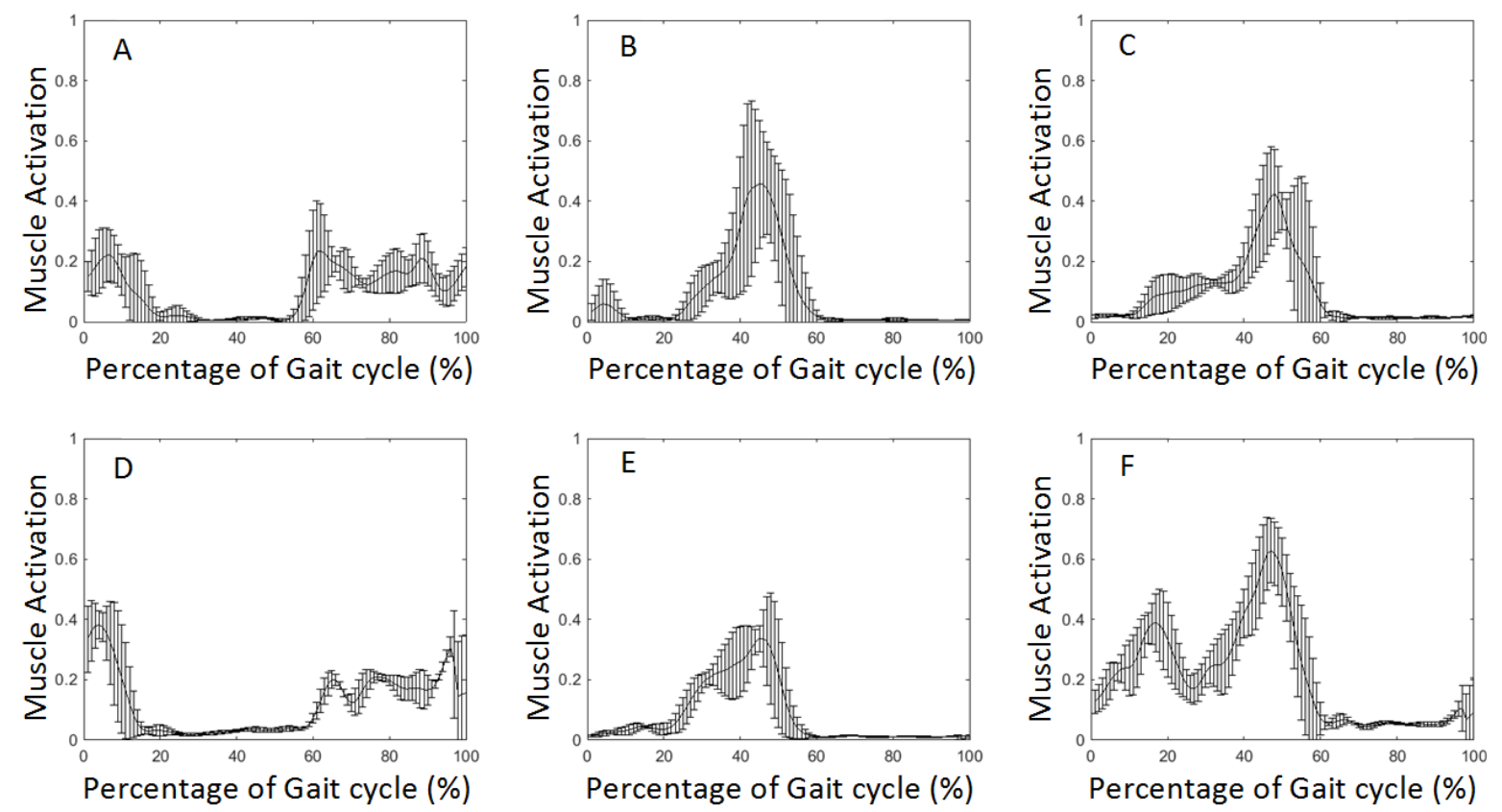

Figure E.23: EMG output of the walking trial with the exoskeleton with PAM inflated to $40 \mathrm{kPa}$ as the spring element. For participant 1 

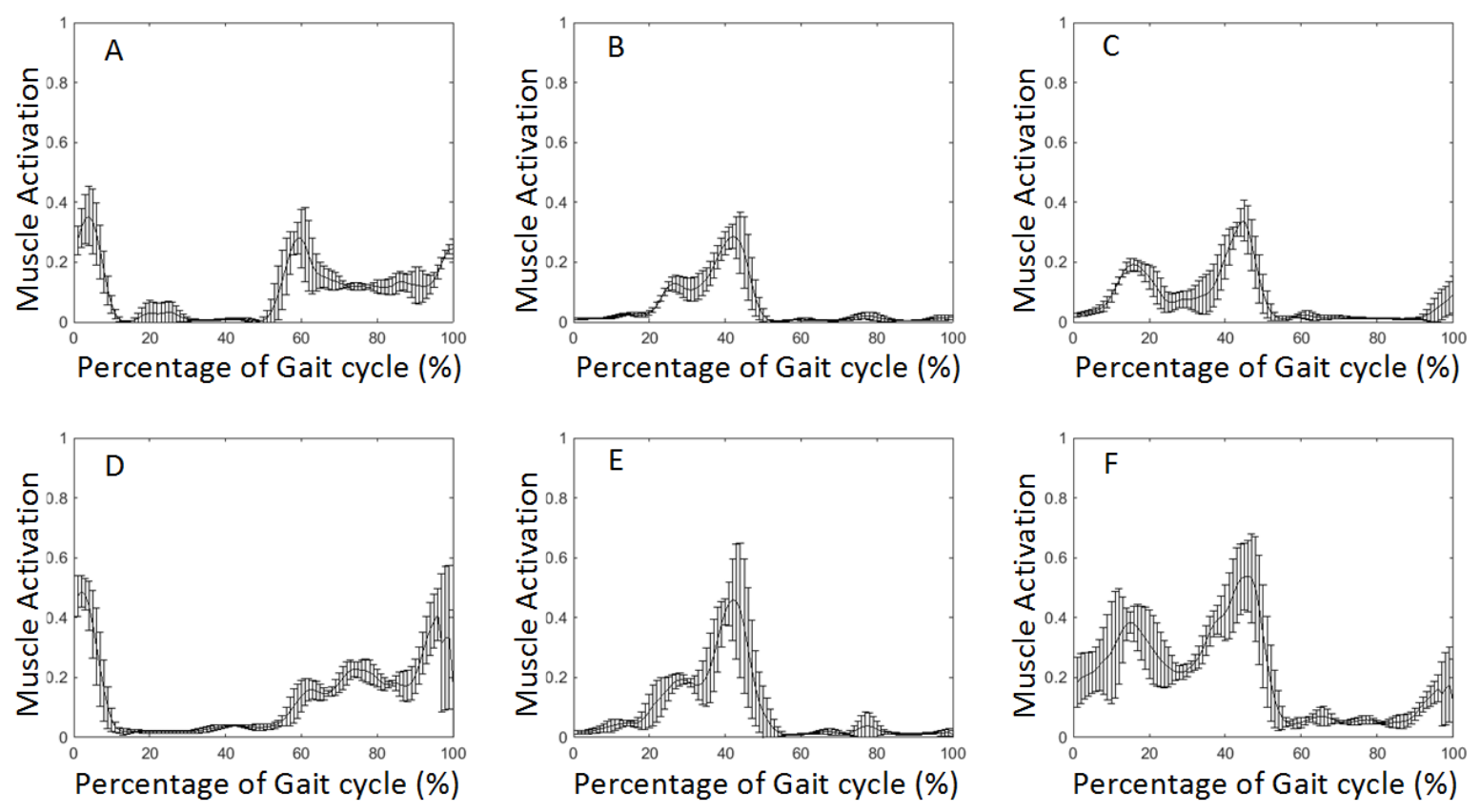

Figure E.24: EMG output of the walking trial with the exoskeleton with PAM inflated to $70 \mathrm{kPa}$ as the spring element. For participant 1

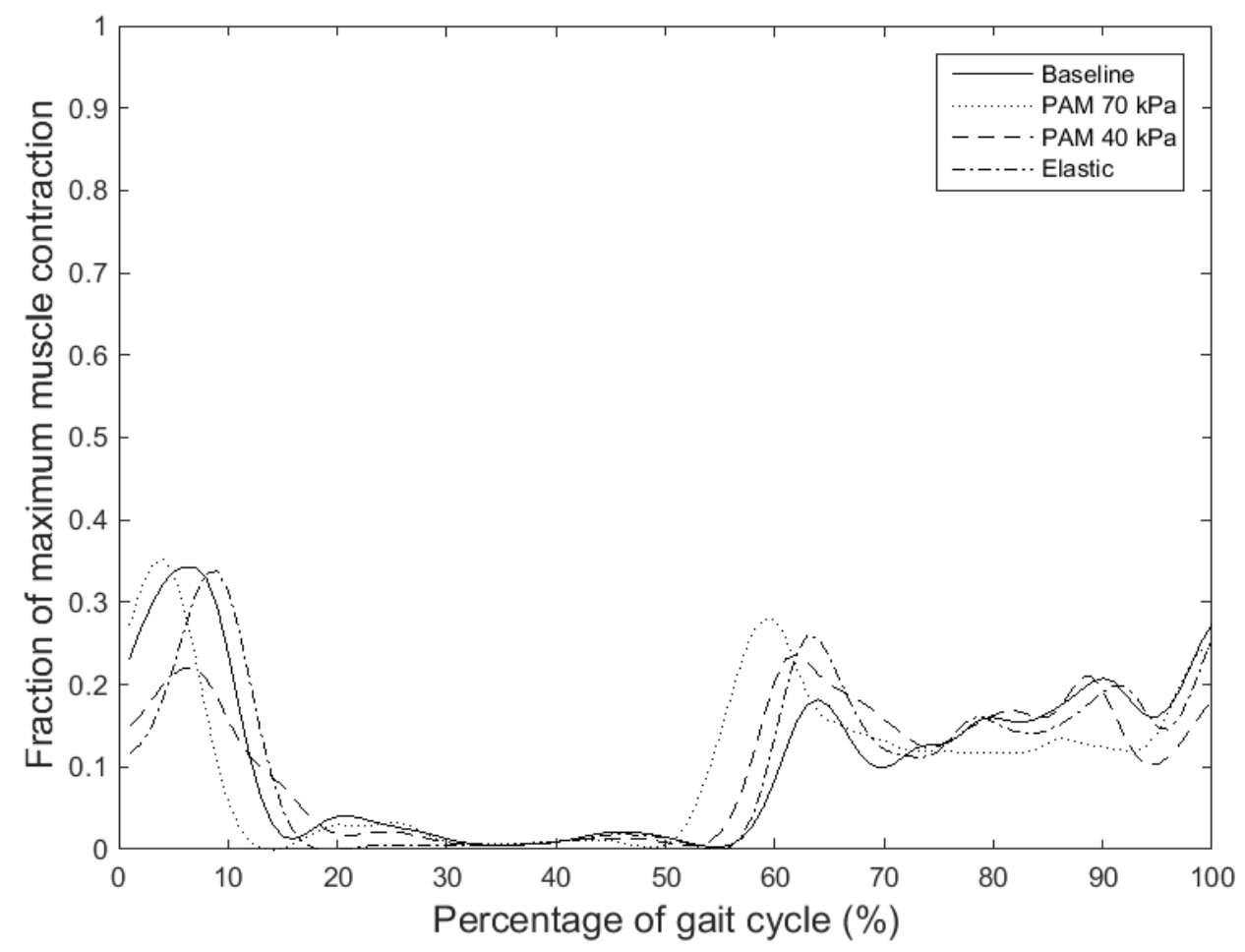

Figure E.25: Comparison of the muscle activation in the Right Tibialis Anterior during the PAM $70 \mathrm{kPa}$, PAM $40 \mathrm{kPa}$, Elastic, and baseline trials for participant 1.

The exoskeleton was worn on the Right leg 


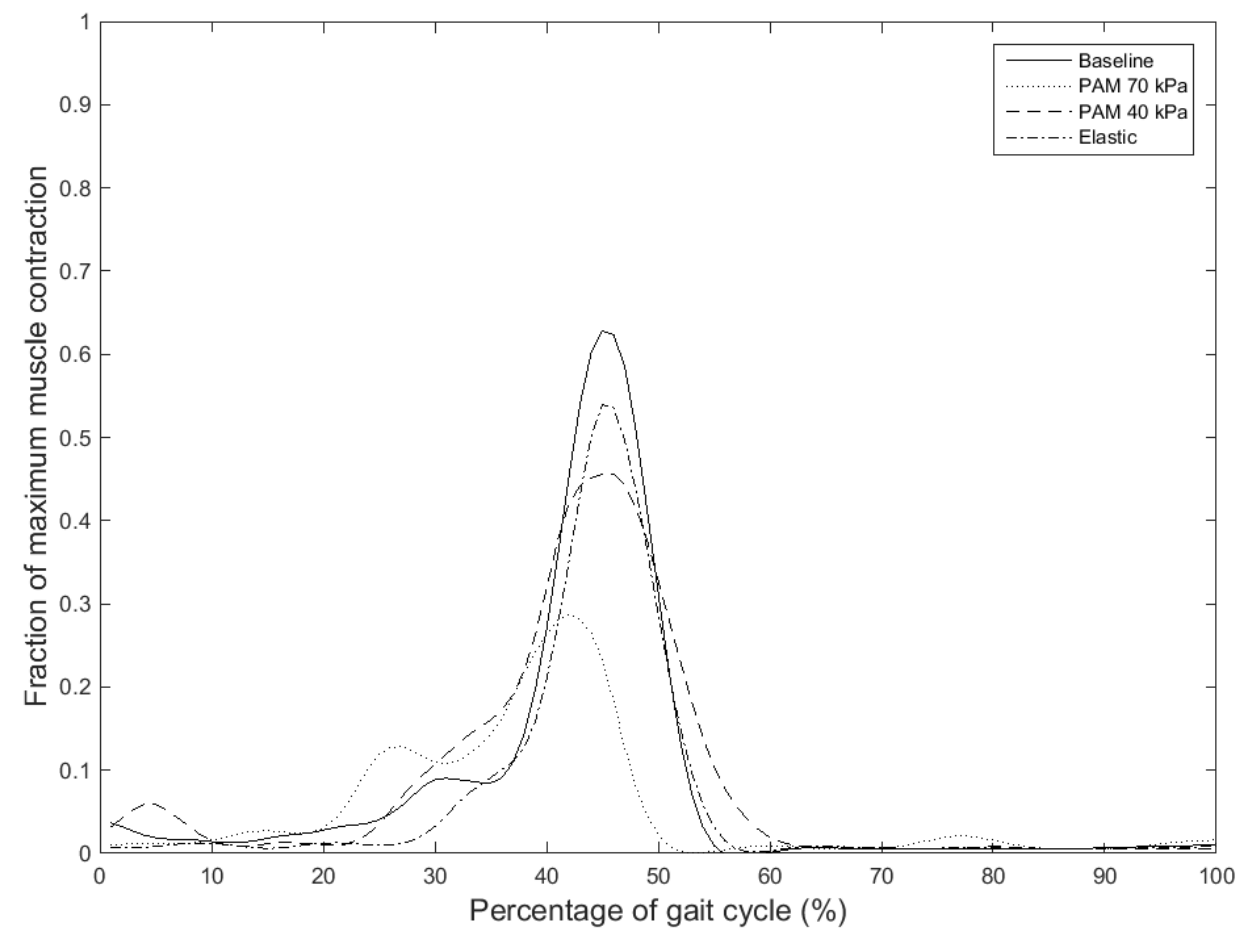

Figure E.26: Comparison of the muscle activation in the Right Gastrocnemius Medialis during the PAM $70 \mathrm{kPa}, \mathrm{PAM} 40 \mathrm{kPa}$, Elastic, and baseline trials for participant 1 . The exoskeleton was worn on the Right leg 


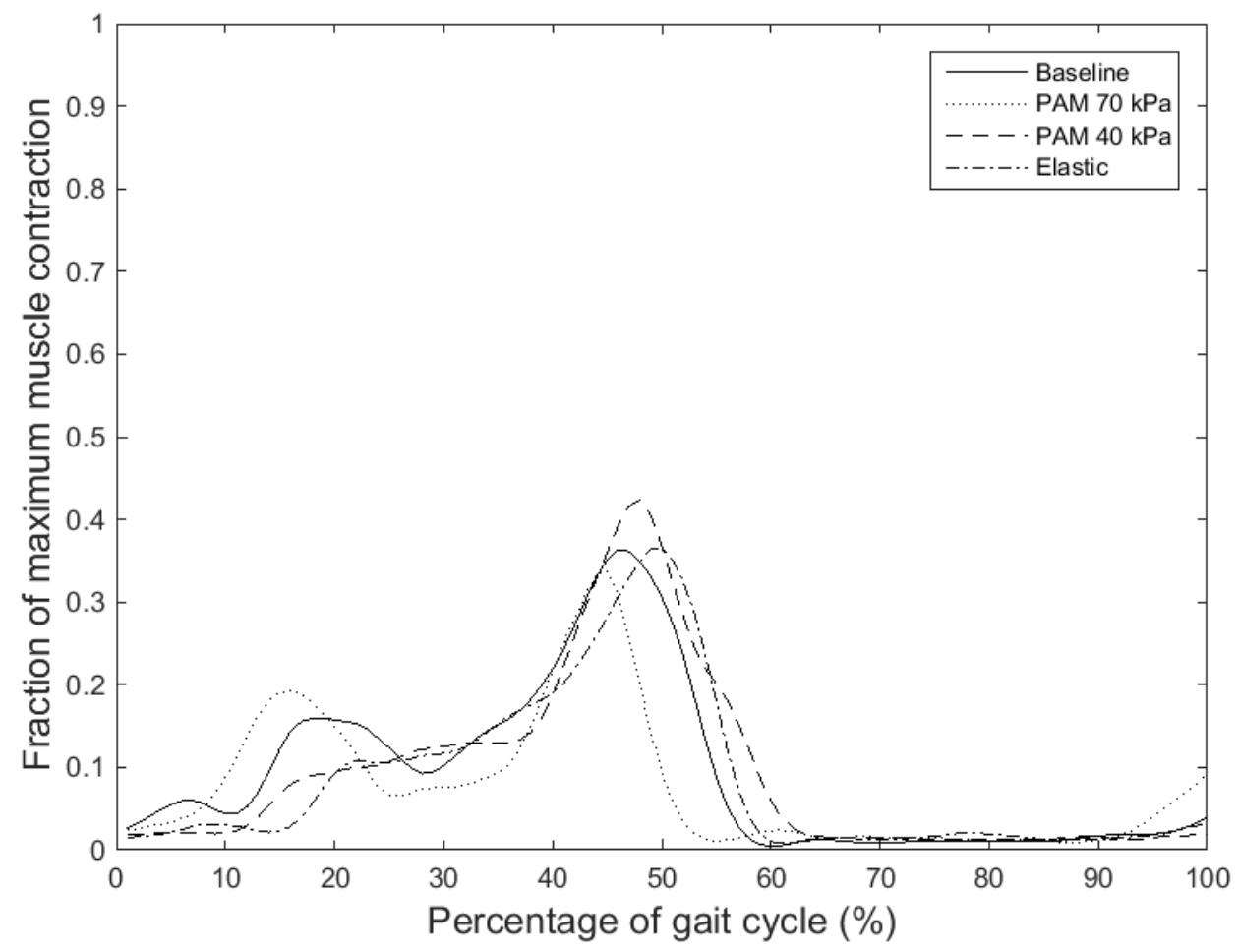

Figure E.27: Comparison of the muscle activation in the Right Soleus during the PAM $70 \mathrm{kPa}$, PAM $40 \mathrm{kPa}$, Elastic, and baseline trials for participant 1. The exoskeleton was worn on the Right leg 


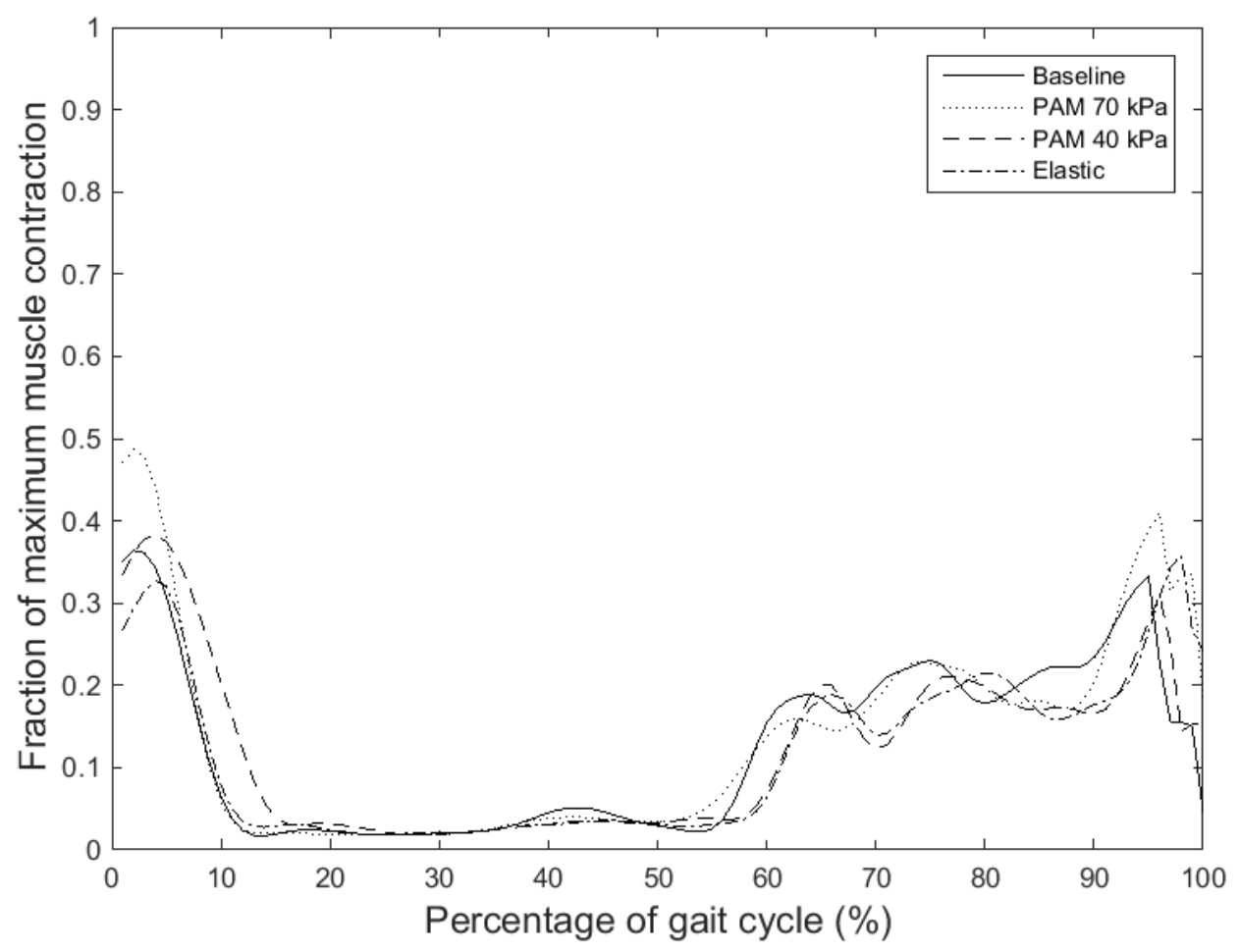

Figure E.28: Comparison of the muscle activation in the Left Tibialis Anterior during the PAM $70 \mathrm{kPa}$, PAM $40 \mathrm{kPa}$, Elastic, and baseline trials for participant 1. The exoskeleton was worn on the Right leg 


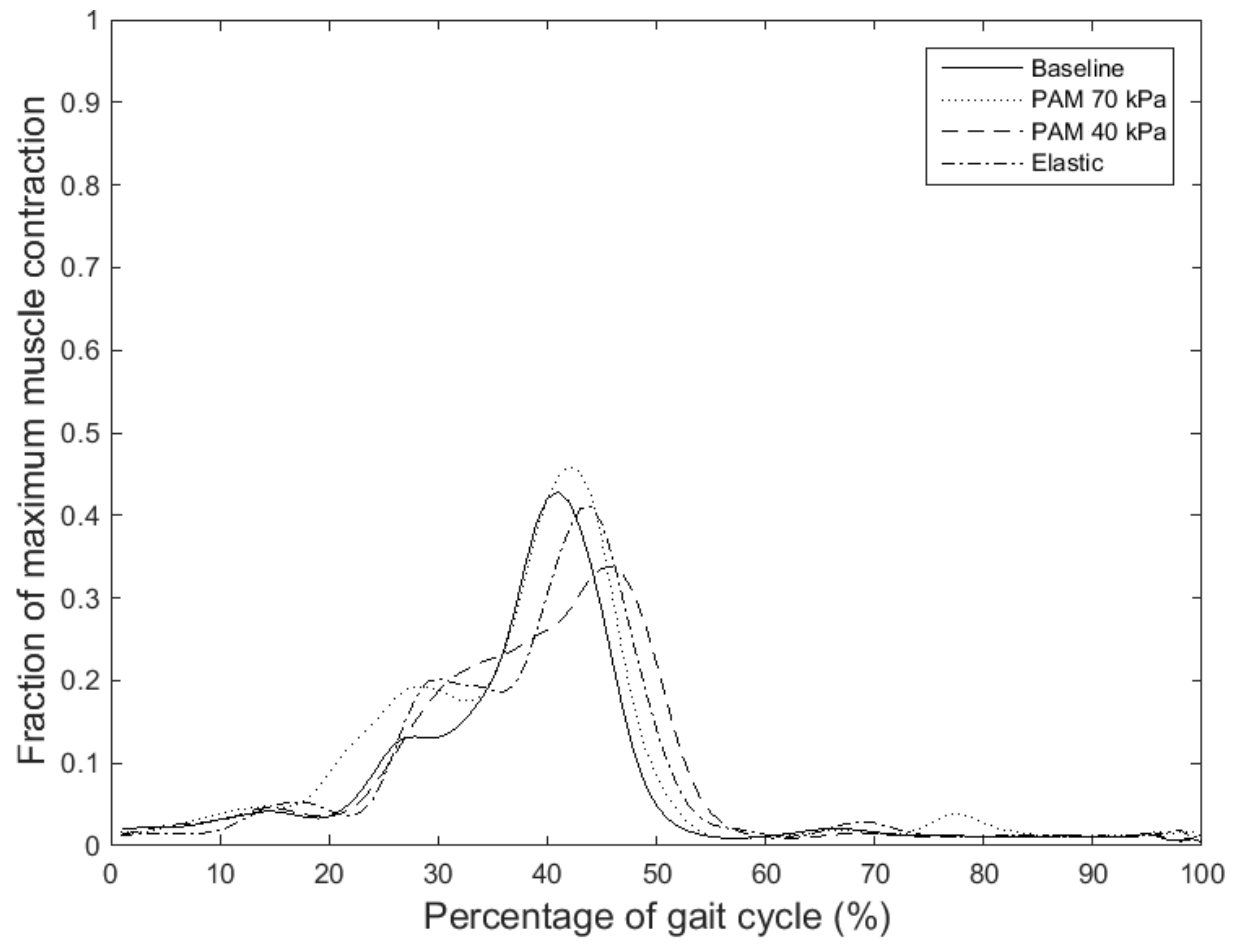

Figure E.29: Comparison of the muscle activation in the Left Gastrocnemius Medialis during the PAM $70 \mathrm{kPa}$, PAM $40 \mathrm{kPa}$, Elastic, and baseline trials for participant 1. The exoskeleton was worn on the Right leg 


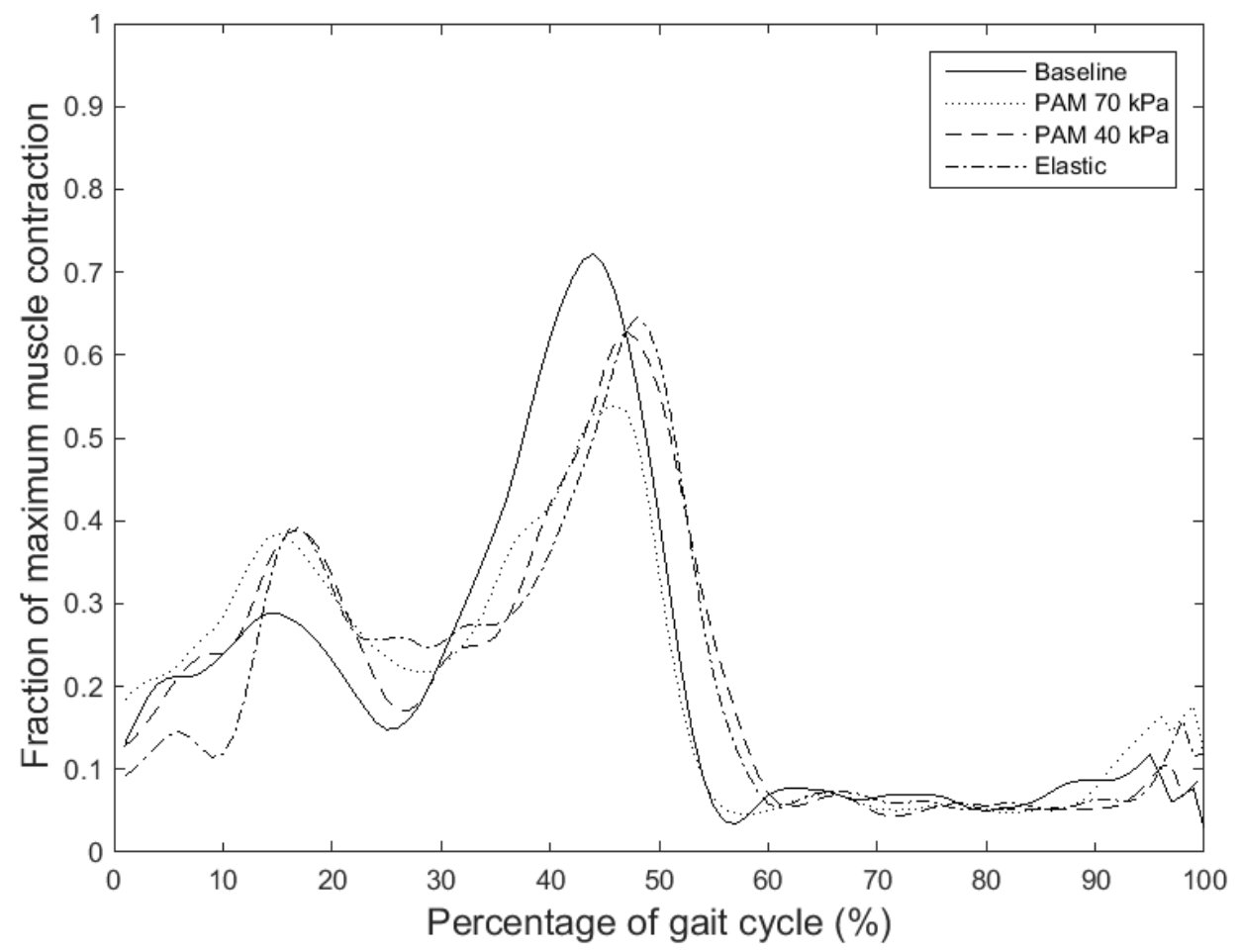

Figure E.30: Comparison of the muscle activation in the Left Soleus during the PAM $70 \mathrm{kPa}$, PAM $40 \mathrm{kPa}$, Elastic, and baseline trials for participant 1 . The exoskeleton was worn on the Right leg 
Appendix F

Ethics Certificate 


\title{
Université d'Ottawa University of Ottawa
}

Bureau d'éthique et d'intégrité de la recherche

Office of Research Ethics and Integrity

This is to confirm that the University of Ottawa Research Ethics Board identified above, which operates in accordance with the Tri-Council Policy Statement and other applicable laws and regulations in Ontario, has examined and approved the application for ethical approval for the above named research project as of the Ethics Approval Date indicated for the period above and subject to the conditions listed the section above entitled "Special Conditions / Comments".

During the course of the study the protocol may not be modified without prior written approval from the REB except when necessary to remove participants from immediate endangerment or when the modification(s) pertain to only administrative or logistical components of the study (e.g. change of telephone number).

Investigators must also promptly alert the REB of any changes which increase the risk to participant(s), any changes which considerably affect the conduct of the project, all unanticipated and harmful events that occur, and new information that may negatively affect the conduct of the project and safety of the participant(s). Modifications to the project, information/consent documentation, and/or recruitment documentation, should be submitted to this office for approval using the "Modification to research project" form available at: http://research.uottawa.ca/ethics/submissions-and-reviews.

Please submit an annual status report to the Protocol Officer 4 weeks before the above-referenced expiry date to either close the file or request a renewal of ethics approval. This document can be found at:

http://research.uottawa.ca/ethics/submissions-and-reviews.

If you have any questions, please do not hesitate to contact the Ethics Office at extension 5387 or by e-mail at: ethics@uOttawa.ca.

\author{
Germain Zongo \\ Protocol Officer for Ethics in Research \\ For Daniel Lagarec, Chair of the Sciences and Health Sciences REB
}

\author{
550, rue Cumberland 550 Cumberland Street \\ Ottawa (Ontario) K1N 6N5 Canada Ottawa, Ontario K1N 6N5 Canada \\ (613) 562-5387 • Téléc./Fax (613) 562-5338 \\ http://www.recherche.uottawa.ca/deontologie/ http://www.research.uottawa.ca/ethics/index.html
}




\section{Appendix G}

\section{MatLab Simulations and data processing code}

\section{G.1 Simulink Simulation}

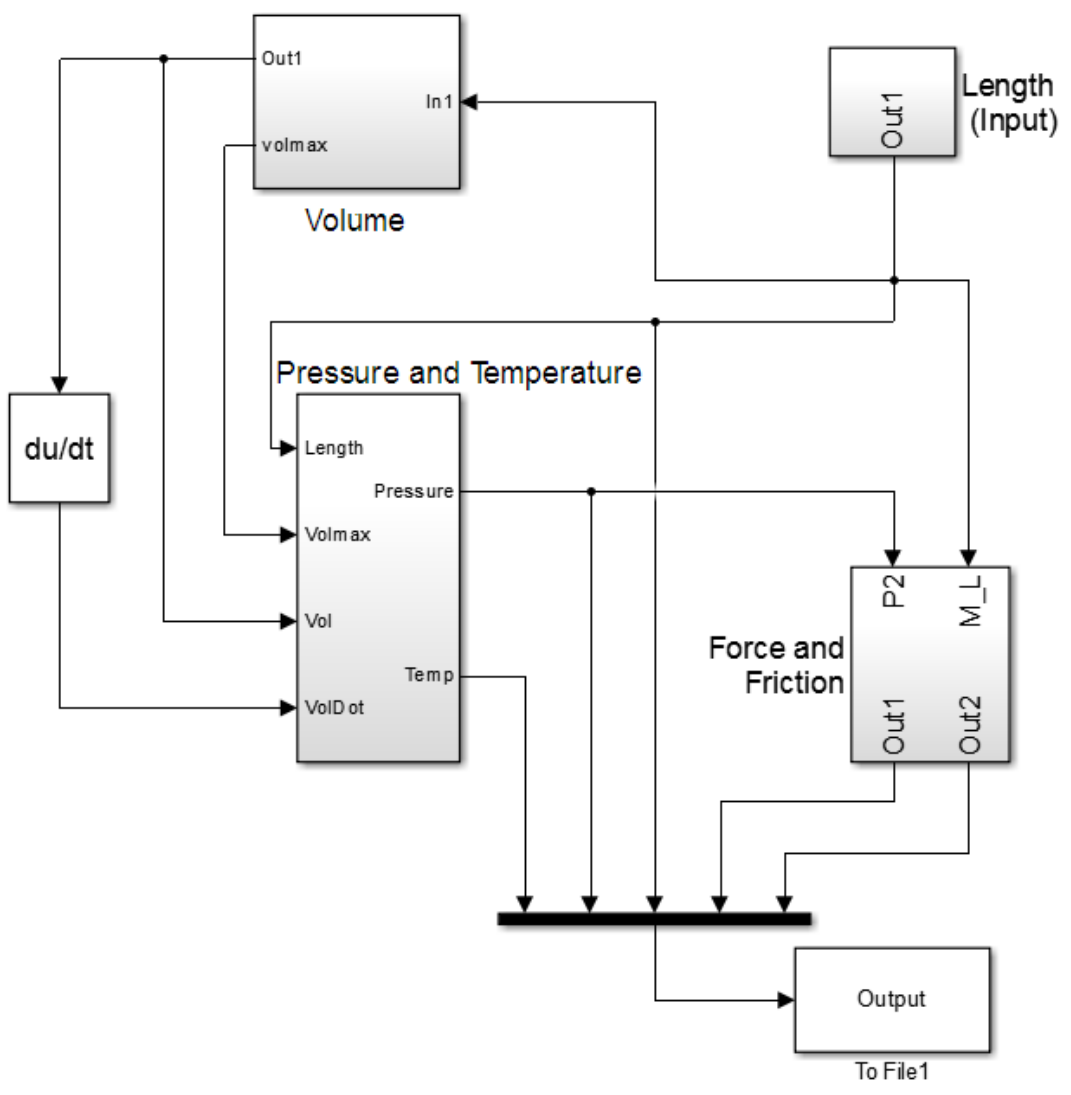

Figure G.1: Image of the Simulink MatLab simulation block diagram 


\section{G.2 Comparison of PAM Simulation and Experimental Testing Script}




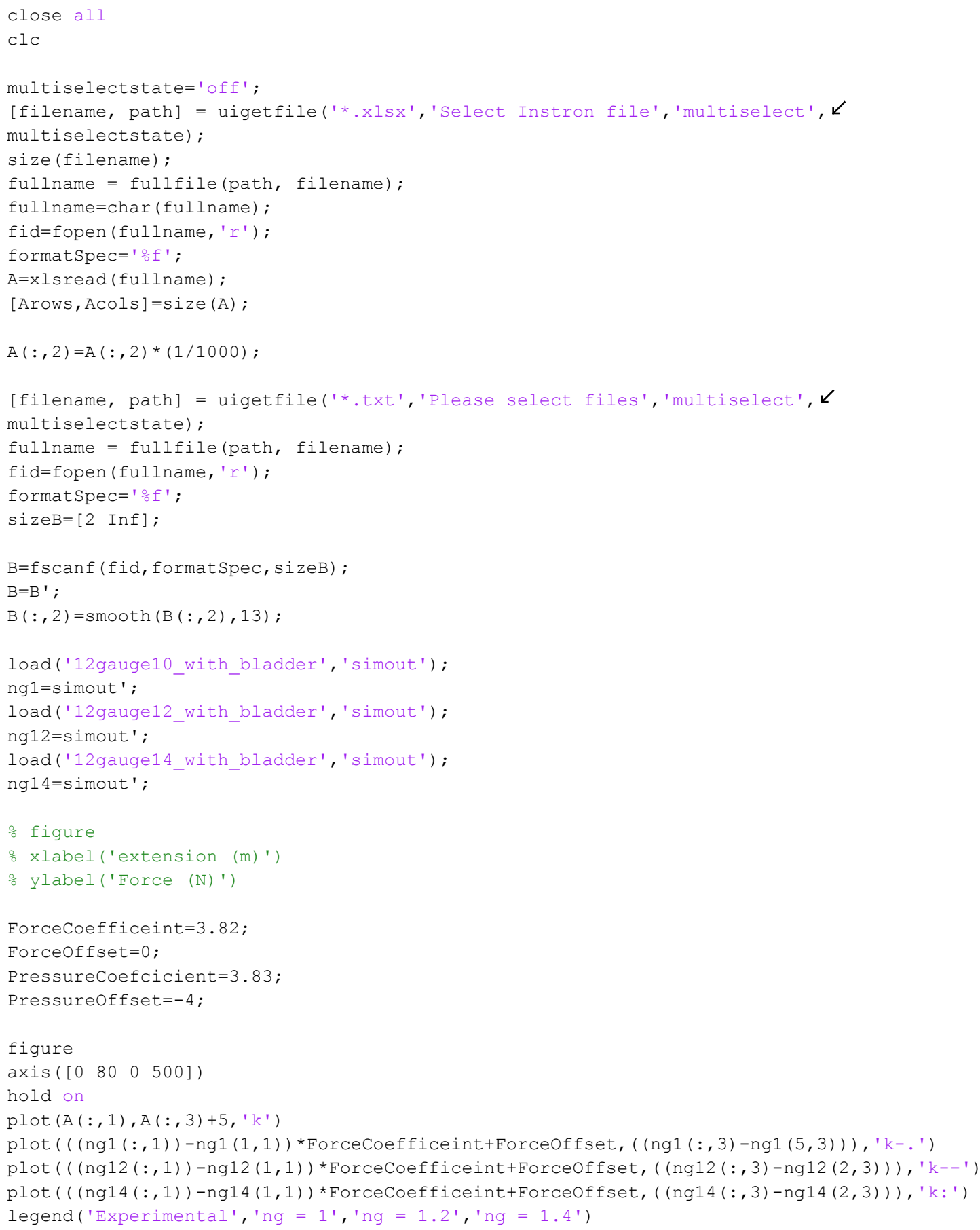




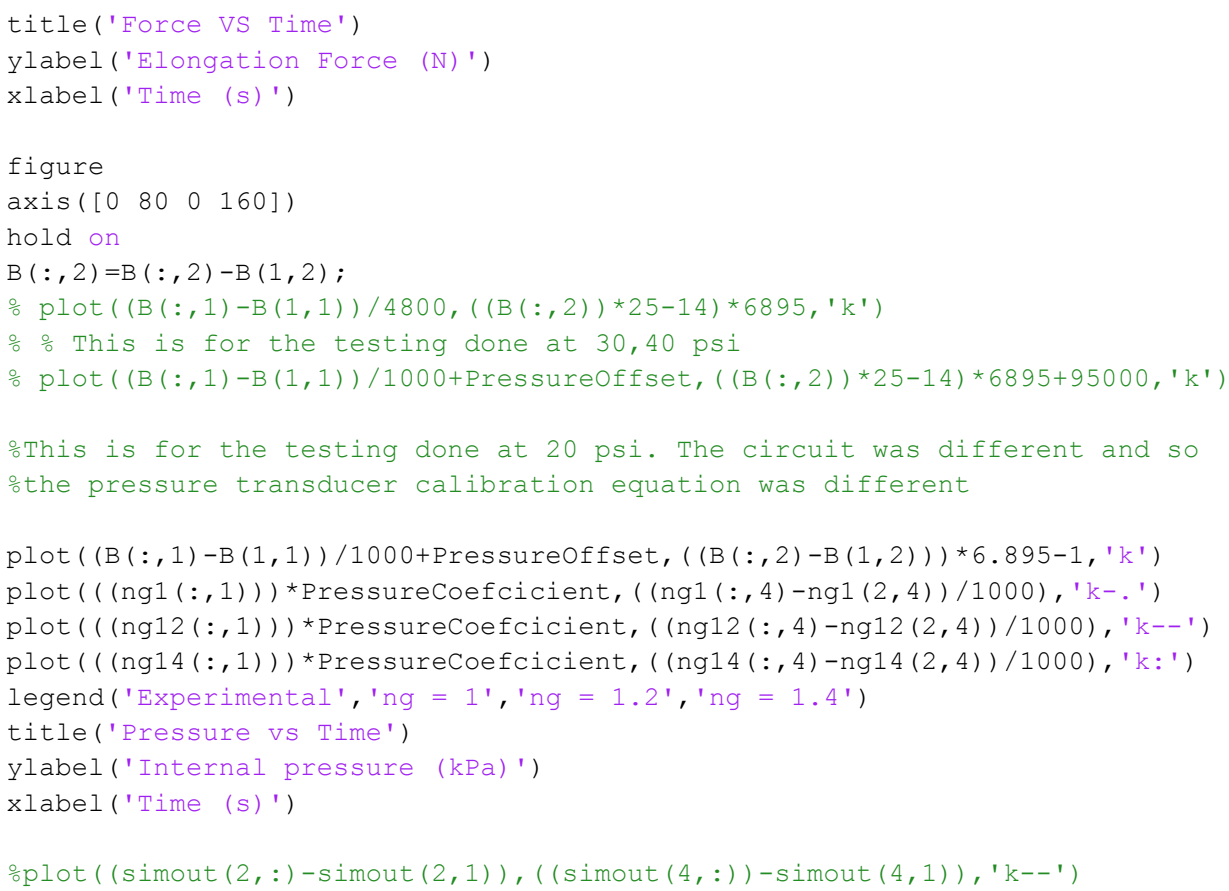




\section{G.3 Kinematic Simulation of Exoskeleton Script}


04/05/17 5:27 PM C:\Users...Vachilles length and moment3.m 1 of 7

$\mathrm{ClC}$

close all

\% create arrays with the data from Winters walking trial for a $56.7 \mathrm{Kg}$

: female individual

응 Winter movement Data

hipangleraw $=\swarrow$

$[112.100000000000,111.300000000000,110.800000000000,110.300000000000,110,109.600000000000$ K $, 109.300000000000,109,108.600000000000,108.400000000000,108.200000000000,107.900000000000 \swarrow$ $, 107.600000000000,106.900000000000,105.900000000000,104.500000000000,102.900000000000,101 K$ $.200000000000,99.3000000000000,97.6000000000000,95.9000000000000,94.3000000000000,92.8000$ L $000000000,91.4000000000000,90,88.7000000000000,87.4000000000000,86,84.6000000000000,83.10 \swarrow$ $00000000000,81.7000000000000,80.2000000000000,78.8000000000000,77.5000000000000,76.300000$ K $0000000,75.3000000000000,74.5000000000000,73.8000000000000,73.4000000000000,73.1000000000 \swarrow$ $000,73.1000000000000,73.4000000000000,74.1000000000000,75,76.4000000000000,78.10000000000 \swarrow$ $00,80.2000000000000,82.5000000000000,85.2000000000000,88.2000000000000,91.3000000000000,9 \swarrow$ $4.5000000000000,97.7000000000000,100.700000000000,103.600000000000,106.100000000000,108.3 K$ $00000000000,110.100000000000,111.700000000000,113,114,114.900000000000,115.400000000000,1 K$ $15.700000000000,115.700000000000,115.400000000000,114.800000000000,114,113.200000000000,1 K$ $12.400000000000,111.700000000000,111.300000000000,110.900000000000,110.800000000000,110.6 \swarrow$ $00000000000,110.500000000000,110.400000000000,110.300000000000,110.200000000000,110,109.6 \swarrow$ $00000000000,109,108.100000000000,107]$;

legangleraw $=\swarrow$

$[107.800000000000,109.700000000000,110.700000000000,110.700000000000,109.800000000000,108 K$ $.400000000000,106.600000000000,104.600000000000,102.500000000000,100.300000000000,98.1000 K$ $000000000,95.9000000000000,93.8000000000000,91.9000000000000,90.3000000000000,88.90000000 K$ $00000,87.6000000000000,86.5000000000000,85.4000000000000,84.4000000000000,83.500000000000$ L $0,82.5000000000000,81.6000000000000,80.6000000000000,79.7000000000000,78.7000000000000,77 \measuredangle$ $.8000000000000,76.8000000000000,75.9000000000000,74.9000000000000,73.8000000000000,72.700 \mathrm{~K}$ $0000000000,71.6000000000000,70.2000000000000,68.7000000000000,67.1000000000000,65.2000000 K$ $000000,63.2000000000000,61.1000000000000,58.7000000000000,56.2000000000000,53.60000000000 K$ $00,50.9000000000000,48.1000000000000,45.4000000000000,42.9000000000000,40.7000000000000,3 \mathbf{K}$ $8.9000000000000,37.6000000000000,36.8000000000000,36.8000000000000,37.4000000000000,38.80 \swarrow$ $00000000000,40.9000000000000,43.6000000000000,46.8000000000000,50.6000000000000,54.600000 \swarrow$ $0000000,59,63.6000000000000,68.3000000000000,73.1000000000000,78,82.9000000000000,87.9000$ L $000000000,92.8000000000000,97.6000000000000,102,105.900000000000,109,111,112,112,111.1000 \swarrow$ $00000000,109.600000000000,107.700000000000,105.600000000000,103.400000000000,101,98.70000 \measuredangle$ $00000000,96.4000000000000,94.2000000000000,92.3000000000000,90.7000000000000]$;

footangleraw $=\swarrow$

$[172.600000000000,174.800000000000,175.800000000000,175.500000000000,174.100000000000,171 K$ $.700000000000,168.400000000000,164.600000000000,160.600000000000,157,154,151.900000000000 \swarrow$ $, 150.600000000000,149.900000000000,149.700000000000,149.700000000000,149.700000000000,149 \swarrow$ $.600000000000,149.500000000000,149.300000000000,149,148.600000000000,148,147.200000000000$ K $, 146.500000000000,145.800000000000,145.200000000000,144.700000000000,144.200000000000,143 \boldsymbol{L}$ $.700000000000,143.100000000000,142.400000000000,141.600000000000,140.600000000000,139.200 \mathrm{~K}$ $000000000,137.400000000000,135.100000000000,132.200000000000,128.600000000000,124.5000000$ K $00000,119.700000000000,114.400000000000,108.600000000000,102.400000000000,96,89.800000000$ L $0000,84.4000000000000,80.1000000000000,77.5000000000000,76.7000000000000,77.6000000000000 K$ $, 79.9000000000000,83.3000000000000,87.5000000000000,92.1000000000000,97.1000000000000,102 \measuredangle$ 
.$\swarrow$

$400000000000,107.800000000000,113.300000000000,118.800000000000,124.500000000000,130.3000 \boldsymbol{L}$ $00000000,136.100000000000,141.900000000000,147.600000000000,153.300000000000,159,164.4000 \swarrow$ $00000000,169.200000000000,173.200000000000,175.900000000000,177.400000000000,177.50000000 \mathrm{~K}$ $0000,176.100000000000,173.500000000000,169.700000000000,165.500000000000,161.200000000000$ K $, 157.400000000000,154.300000000000,152.100000000000,150.600000000000,149.700000000000,149 \swarrow$ $.300000000000]$;

anklemomentraw $=[-0.500000000000000,-0.300000000000000, \swarrow$

$-1.70000000000000,4.60000000000000,8.30000000000000,2.80000000000000,6.90000000000000,4.6 K$ $0000000000000,5,2.60000000000000,-0.500000000000000,0.200000000000000,-3.50000000000000, K$ $-5.10000000000000,-6.60000000000000,-10.5000000000000,-10.6000000000000, K$ $-14.1000000000000,-18.7000000000000,-22.5000000000000,-25.3000000000000, \swarrow$ $-27.9000000000000,-30.3000000000000,-33.5000000000000,-38.8000000000000, \swarrow$ $-45.1000000000000,-45.4000000000000,-53.5000000000000,-58.8000000000000, \boldsymbol{L}$ $-64.8000000000000,-71.2000000000000,-77.3000000000000,-82.7000000000000,-87, \swarrow$ $-89.7000000000000,-89.8000000000000,-86.7000000000000,-79.7000000000000, \boldsymbol{L}$ $-68.6000000000000,-54.3000000000000,-38.8000000000000,-24.2000000000000, \swarrow$ $-12.3000000000000, \boldsymbol{L}$

$-3.60000000000000,1.40000000000000,1.40000000000000,1.40000000000000,1.30000000000000,1.1 K$ $0000000000000,0.900000000000000,0.700000000000000,0.600000000000000,0.500000000000000,0.5 K$ $00000000000000,0.500000000000000,0.500000000000000,0.600000000000000,0.600000000000000,0 . \swarrow$ $700000000000000,0.800000000000000,0.800000000000000,0.700000000000000,0.600000000000000,0 K$ $.400000000000000,0.100000000000000,-0.200000000000000,-0.400000000000000, \swarrow$ $-0.500000000000000,-0.600000000000000,-0.500000000000000, \swarrow$

$-0.300000000000000,0,0.300000000000000,0.500000000000000,0.600000000000000,0.700000000000 \measuredangle$ $000,0.700000000000000,0.600000000000000,0.600000000000000,0.600000000000000,0.6000000000, K$ $0.60000000,0.60000000,0.60000000]$;

othis section converts angles into Rad and inverts the sign of the moment

hipangle $=($ pi/180 $) *$ hipangleraw + pi;

legangle $=(\mathrm{pi} / 180) *$ legangleraw $+\mathrm{pi}$;

footangle $=(p i / 180) *$ footangleraw + pi;

anklemoment=anklemomentraw* $(-1)$;

응 Setup and variable declaration

:Subject information and dimensions

subjectweight $=56.7$; $\frac{\circ}{\circ} \mathrm{Kg}$

peakanklemoment $=80 * 1.4 ; \circ \mathrm{Nm}$

thighlength $=0.42 ; \div 31.4 \mathrm{~cm}$

shanklength $=0.425 ; \div 42.5 \mathrm{~cm}$

footlength=0.26; 응 $12.2 \mathrm{~cm}$

shankoffset=0.1; $8 \mathrm{~cm}$ horizontal offset of shank anchor

heeloffset=0.17; ohorisontal distance between pivot and anchor

uprightlength=0.3; odistance along shank between ankle and shank anchor arm

blockangle=100*pi/180; \%angle between heel arm and shank strut when device jams and $\boldsymbol{K}$

springs is at minimum length

disengageangle=138*pi/180; \%angle between foot arm and shank strut when mechanism $\boldsymbol{L}$

disengages

engageangle=111*pi/180; oangle between foot arm and shank strut when mechanism re-engages 


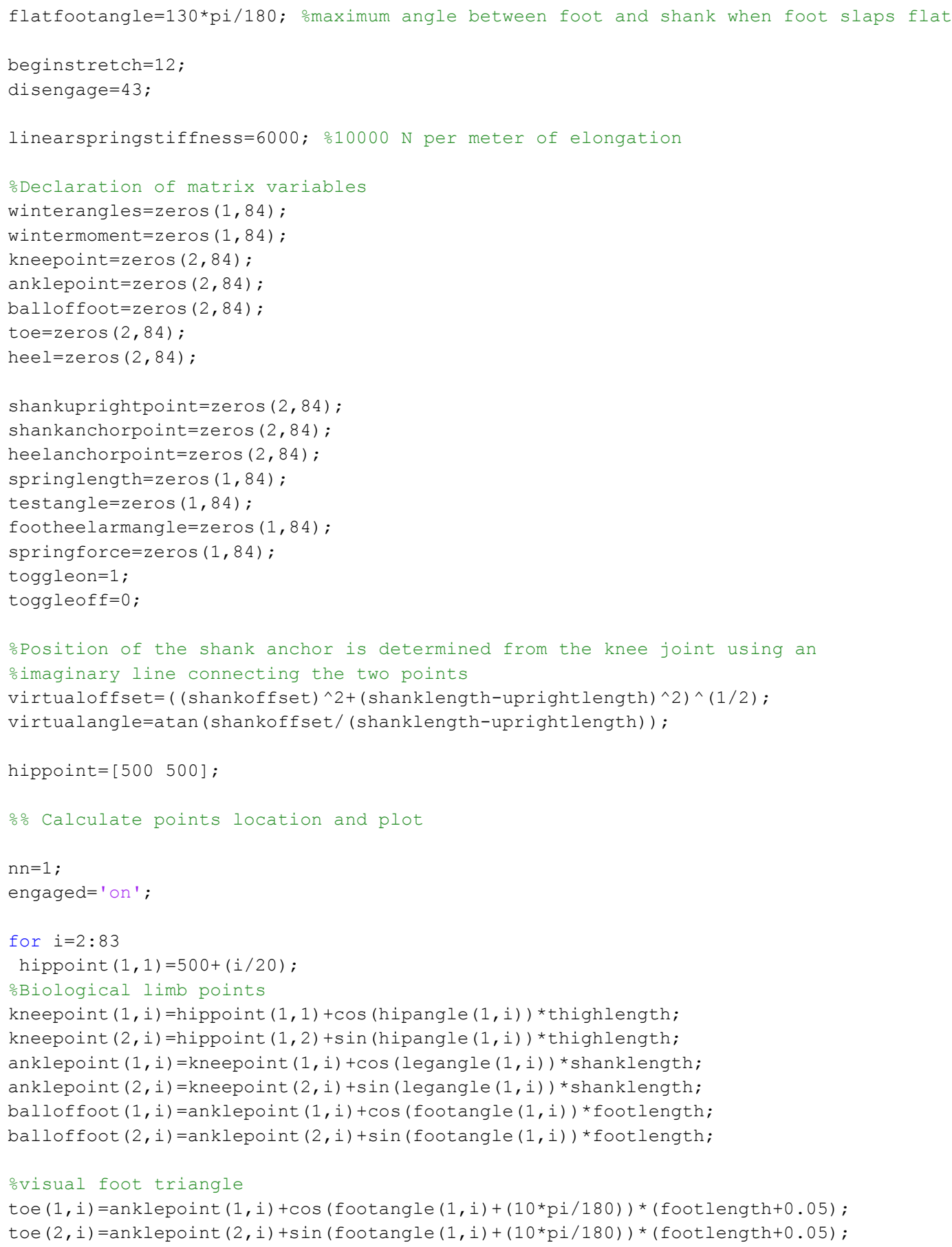


heel $(1, i)=\operatorname{anklepoint}(1, i)+\cos ($ footangle $(1, i)-(90 *$ pi $/ 180)) *(0.1)$;

heel $(2, i)=\operatorname{anklepoint}(2, i)+\sin ($ footangle $(1, i)-(90 *$ pi $/ 180)) *(0.1)$;

oDevice points

\% $\quad$ shankanchorpoint $(1, i)=$ kneepoint $(1, i)+\cos ($ legangle $(1, i)$-virtualangle) *virtualoffset;

o shankanchorpoint $(2, i)=$ kneepoint $(2, i)+\sin ($ legangle $(1, i)$-virtualangle) *virtualoffset; shankuprightpoint $(1, i)=\operatorname{kneepoint}(1, i)+\cos ($ legangle $(1, i)) *($ shanklength-uprightlength) ; shankuprightpoint $(2, i)=k n e e p o i n t(2, i)+\sin ($ legangle $(1, i))$ * (shanklength-uprightlength) ;

shankanchorpoint $(1, i)=$ shankuprightpoint $(1, i)+\cos ($ legangle $(1, i)-(p i / 2))$ *shankoffset; shankanchorpoint $(2, i)=\operatorname{shankuprightpoint}(2, i)+\sin ($ legangle $(1, i)-(p i / 2))$ *shankoffset;

ocheck the shank offset. should be equal to the user input value offsetlength=sqrt ( (shankanchorpoint (1,i)-shankuprightpoint $(1, i))^{\wedge} 2+($ shankanchorpoint $(2, i) \boldsymbol{\swarrow}$ -shankuprightpoint $\left.(2, i))^{\wedge} 2\right)$;

$\therefore \quad \frac{\circ}{\circ} \div$ Engagement and disengagement Timing

footheelangle=footangle ( 1 , beginstretch) - (legangle ( 1 , beginstretch) -pi+blockangle);

if $i>$ beginstretch \&\& i<disengage

plot ([hippoint $(1,1)$ hippoint $(1,1)],[500$ 499.75], 'g')

heelanchorpoint $(1, i)=\operatorname{anklepoint}(1, i)+\cos ($ footangle $(1, i)$-footheelangle) *heeloffset;

heelanchorpoint $(2, i)=a n k l e p o i n t(2, i)+\sin ($ footangle $(1, i)$-footheelangle) *heeloffset;

else

plot([hippoint (1,1) hippoint (1,1) ], [499 499.25], 'r')

heelanchorpoint $(1, i)=a n k l e p o i n t ~(1, i)+\cos ($ legangle $(1, i)-p i+b l o c k a n g l e) *$ heeloffset;

heelanchorpoint $(2, i)=a n k l e p o i n t ~(2, i)+\sin ($ legangle $(1, i)-p i+b l o c k a n g l e) *$ heeloffset; end

testangle $(1, i)=\operatorname{legangle}(1, i)-p i+(2 * p i-f o o t a n g l e(1, i))$;

ㅇminimum spring length

springlength $(1, i)=\operatorname{sqrt}\left((\text { heelanchorpoint }(1, i)-\operatorname{shankanchorpoint}(1, i))^{\wedge} 2+(\right.$ heelanchorpoint $\boldsymbol{K}$ $\left.(2, i)-\operatorname{shankanchorpoint}(2, i))^{\wedge} 2\right)$;

shankanchorpointx=shankanchorpoint $(1, i)$;

shankanchorpointy=shankanchorpoint $(2, i)$;

heelanchorpointx=anklepoint $(1, i)+\cos (l$ egangle $(1, i)-p i+b l o c k a n g l e)$ *heeloffset;

heelanchorpointy=anklepoint $(2, i)+\sin ($ legangle $(1, i)-p i+b l o c k a n g l e)$ *heeloffset;

minspring=sqrt ((heelanchorpointx-shankanchorpointx)^2 + (heelanchorpointy-

shankanchorpointy)^2);

if springlength $(1, i)<$ minspring

: $\quad$ springlength $(1, i)=$ minspring;

\% end 


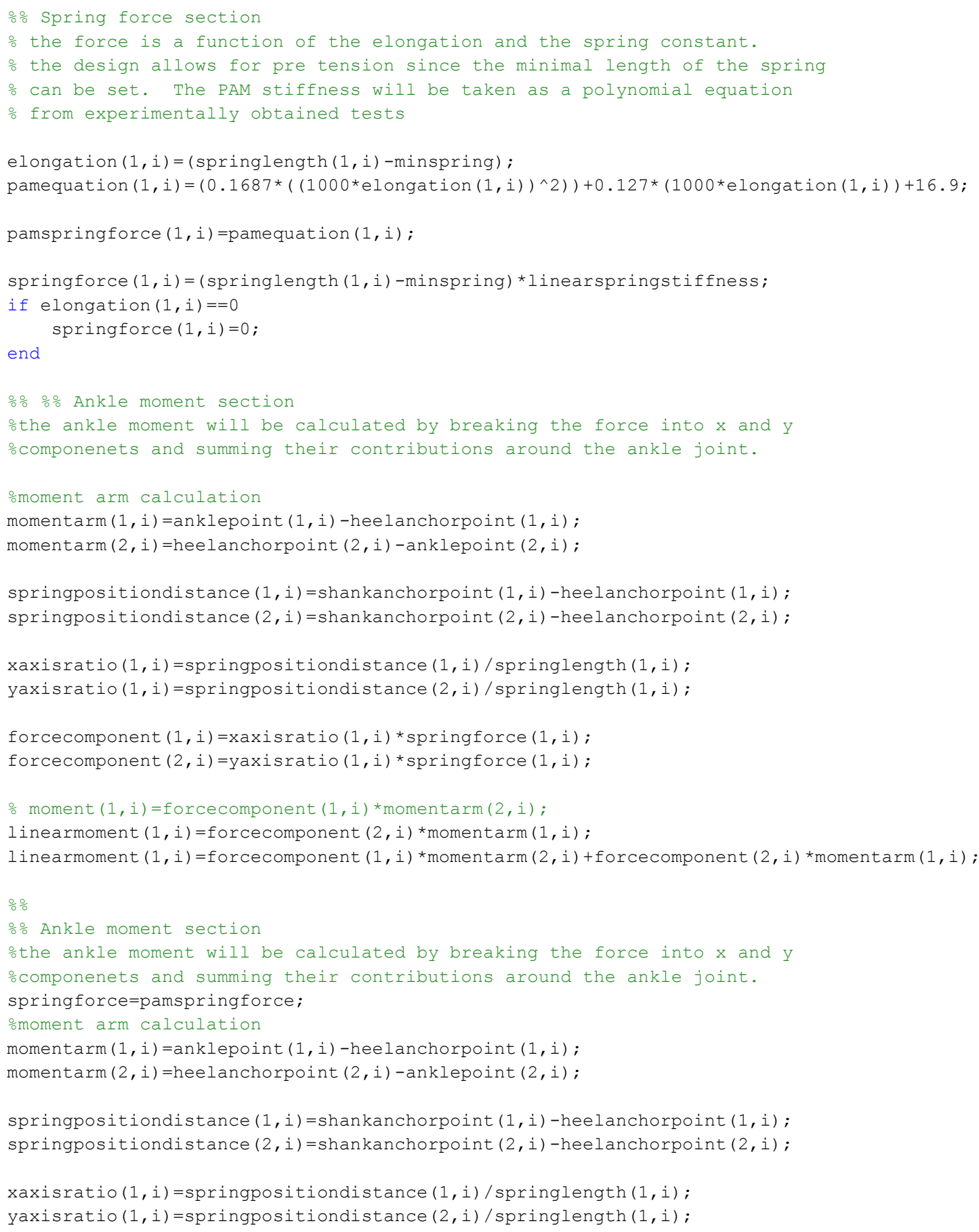




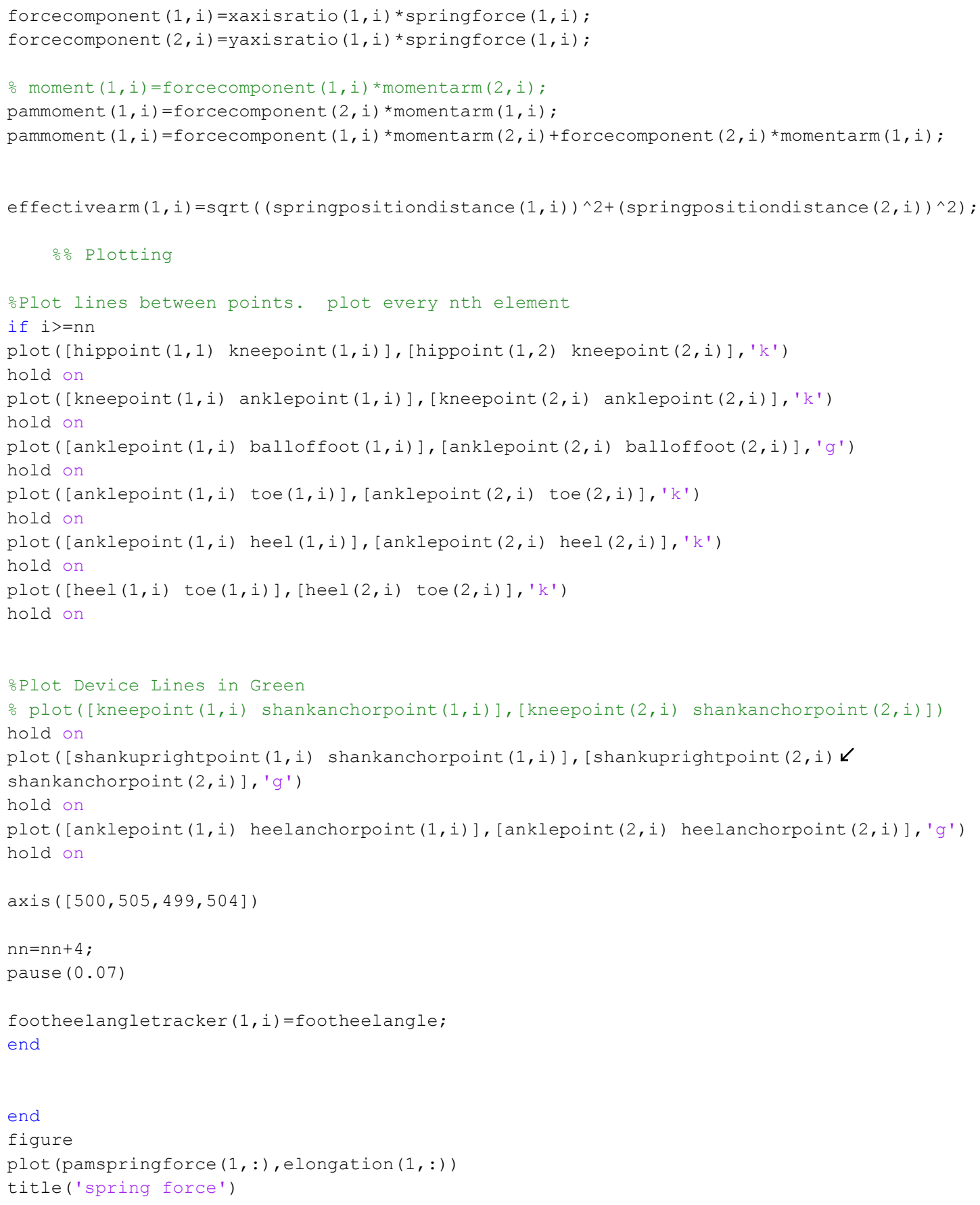




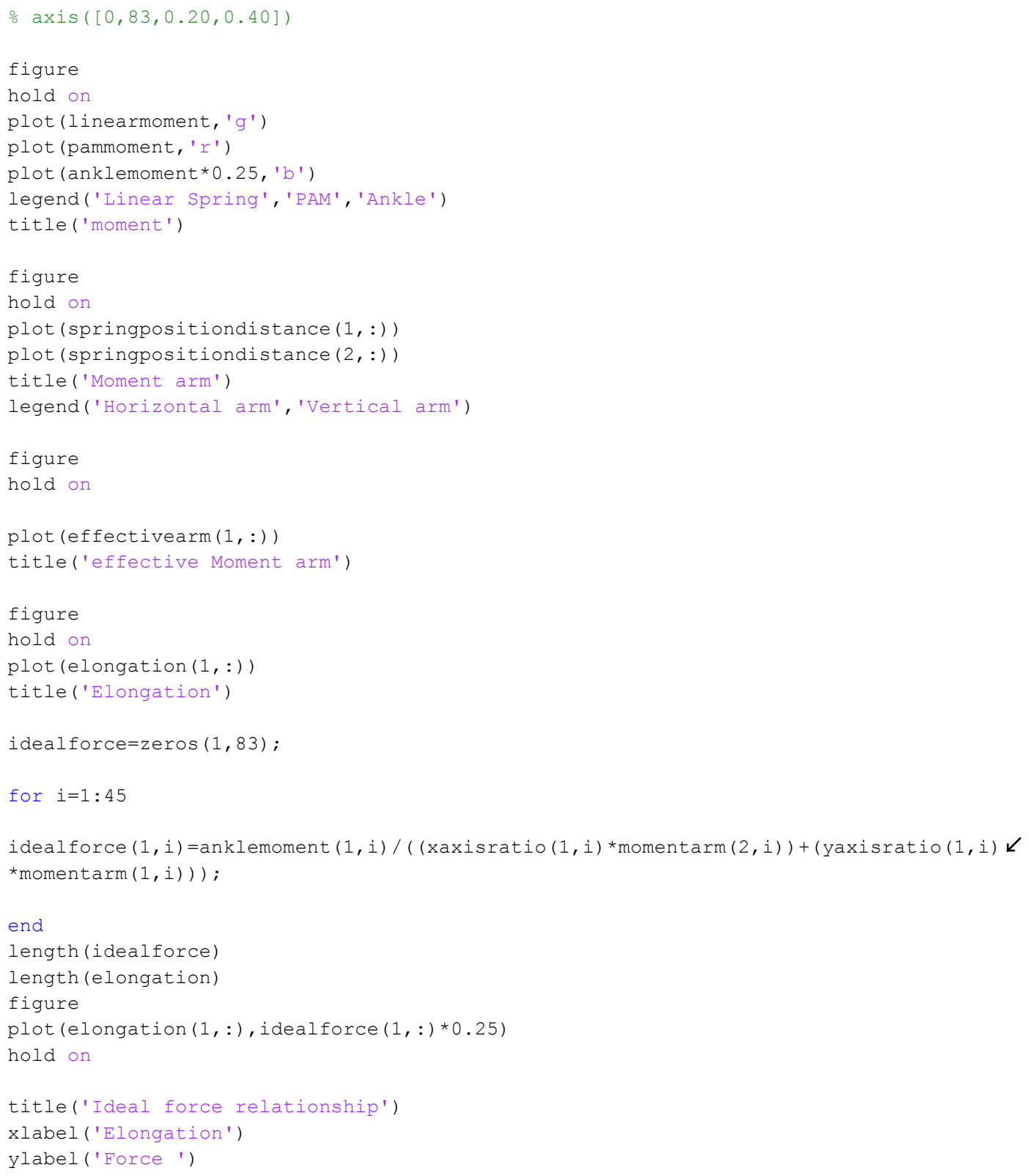




\section{G.4 Mechanical Testing Analysis Script}

The first half of the mechanical testing script is the same as the Kinematic Simulation Exoskeleton Script. In order to not repeat code, only the relevant sections of the mechanical testing code is shown here. 


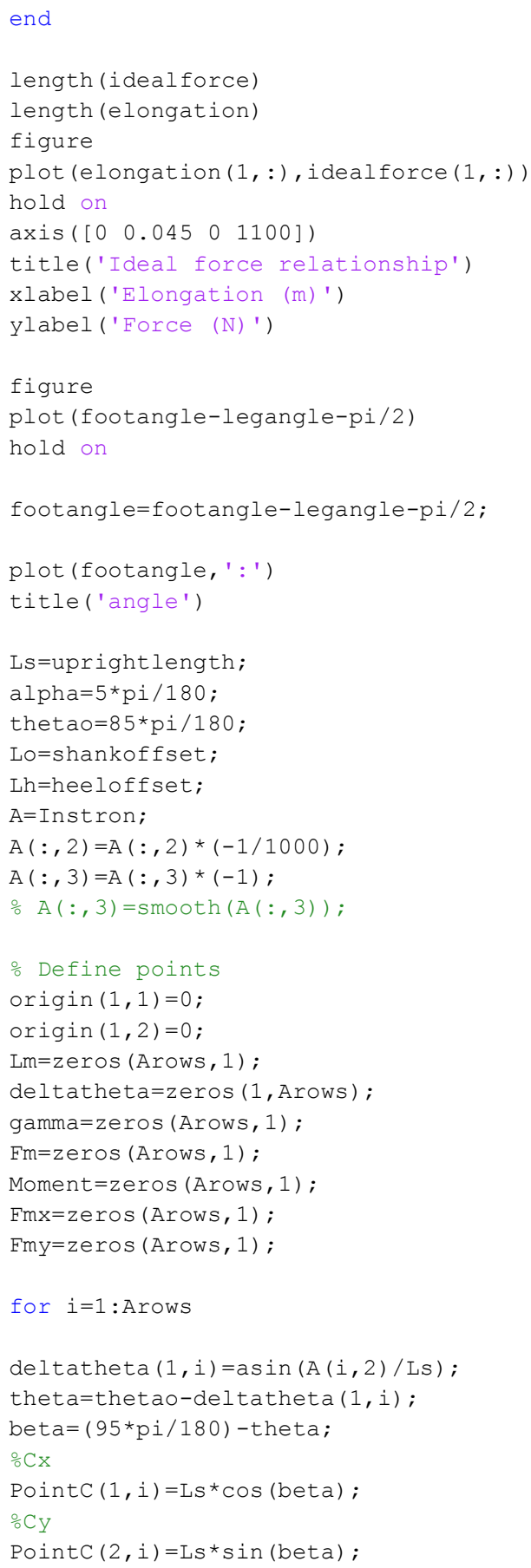




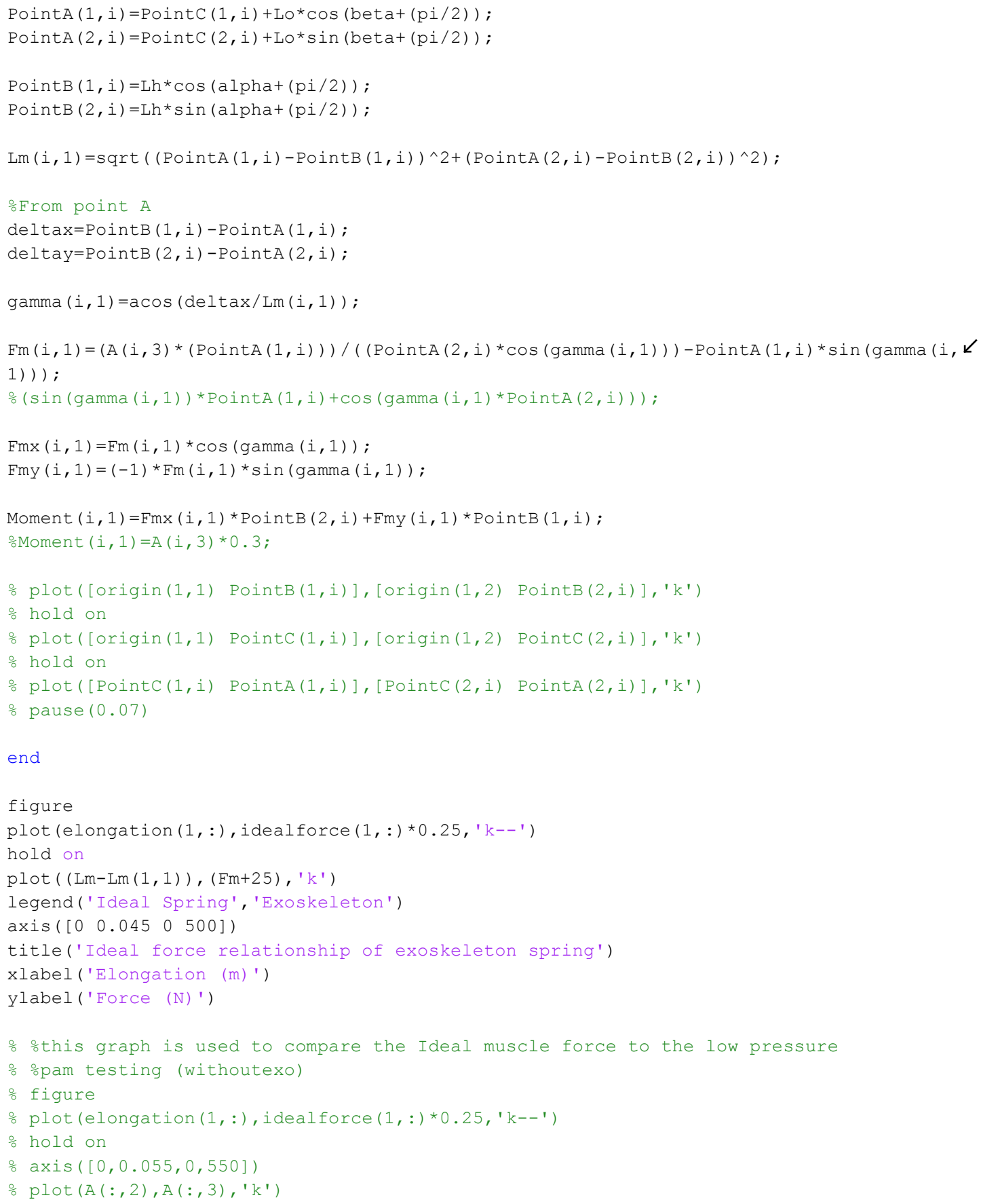




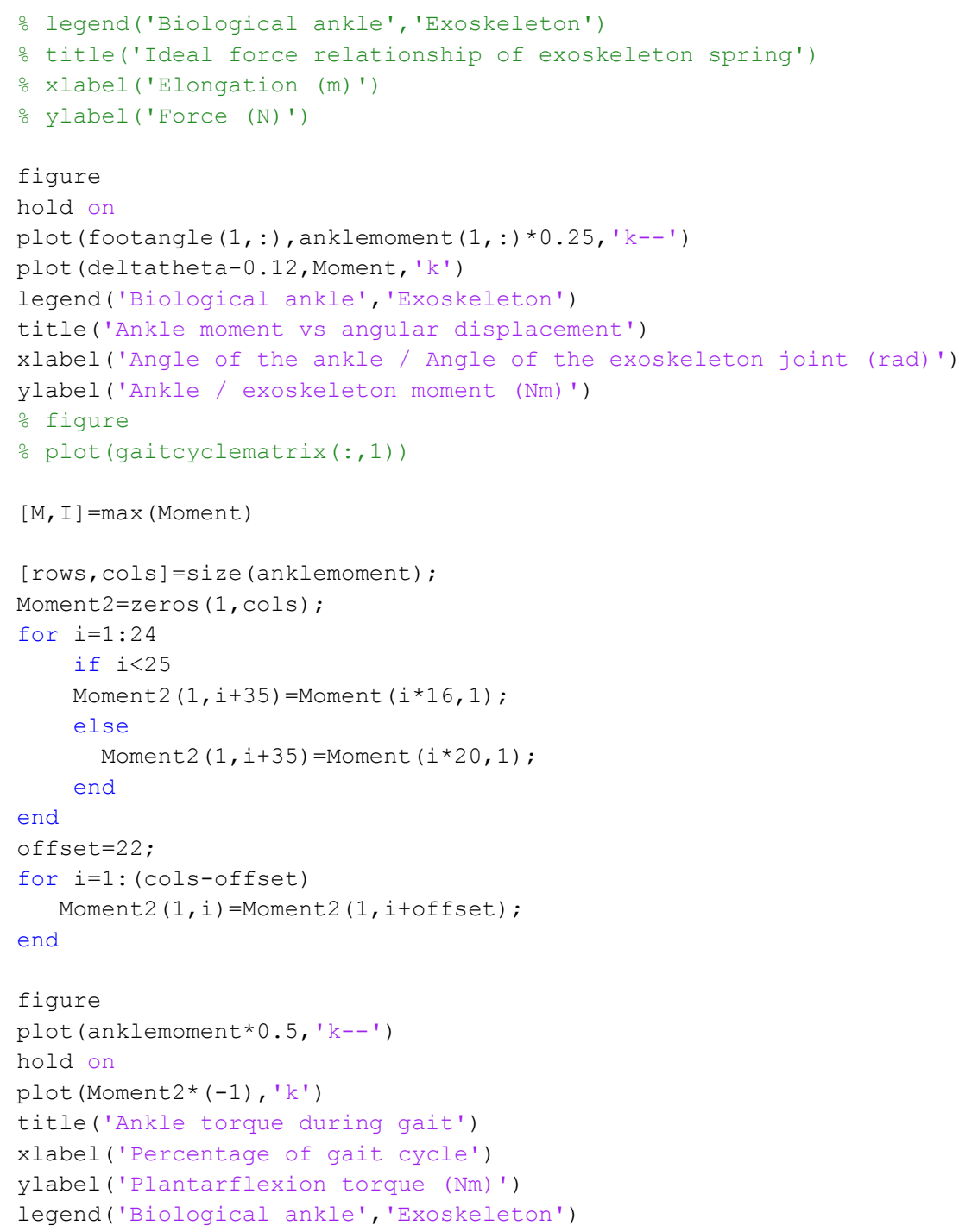




\section{G.5 Human Testing EMG Processing Script}

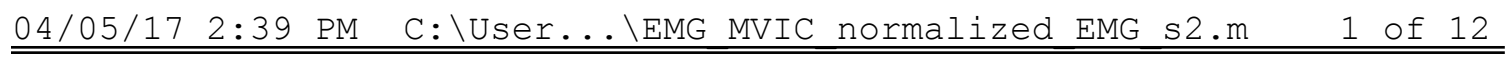

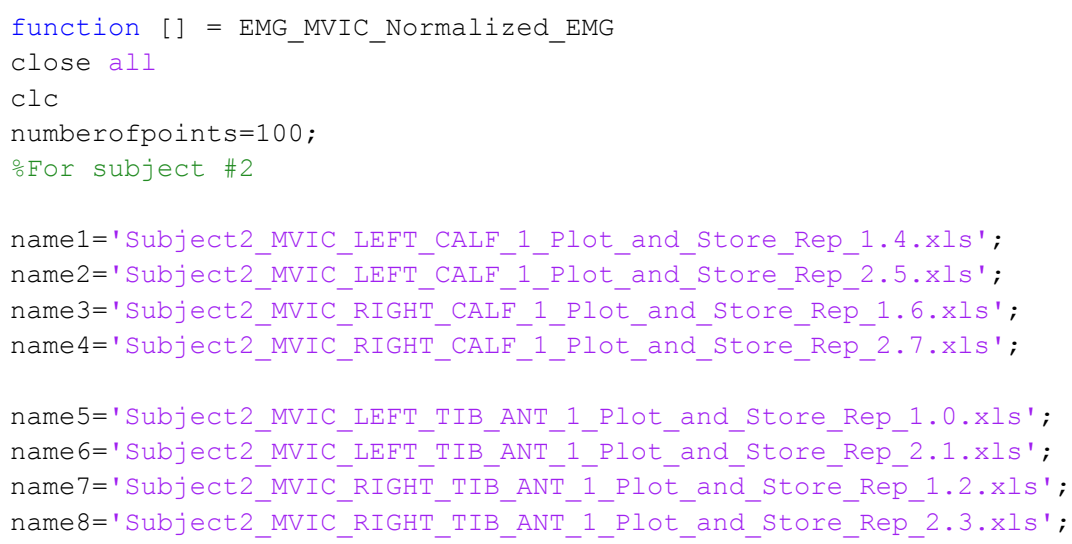


name11='S2_NoEXO_Plot_and_Store_Rep_3.54.CSV'; EventFrames(3, :)=[1.447 $227474578525 \swarrow$ 629]; name12='S2_NoEXO_Plot_and_Store_Rep_4.55.CSV'; EventFrames(4, :)=[1.425 $197465569516 \boldsymbol{L}$ 620];

name13='S2_NoEXO_Plot_and_Store_Rep_5.56.CSV';EventFrames(5, :) =[ $1.464209451554502 \boldsymbol{\swarrow}$ $607]$;

name14='S2_NoEXO_Plot_and_Store_Rep_7.58.CSV'; EventFrames(6, :)=[1.392 $193448550500 \boldsymbol{\swarrow}$ $604]$;

name15='S2_NoEXO_Plot_and_Store_Rep_8.59.CSV';EventFrames(7, : )=[1.18 $169420523470 \swarrow$ 576] ;

OpenFilterAndProcess 7 (name1, name2, name3, name4, name5, name6, name7, name8, name9, name10, $\boldsymbol{k}$ name11, name12, name13, name14, name15, EventFrames, numberofpoints,title1) ;

title1='Walking Exo 6 psi';

EventFrames=zeros $(7,6)$;

ong frame (header already subtracted, Mocap initial frame, R heel heel, L

oheel heel

name9='Subject 1 Walking PAM6 001 Plot and Store Rep 1.11.CSv'; EventFrames (1, : )=[1.325 $\boldsymbol{}$ $40964776558 \overline{9} \overline{7} 10]$;

name10='Subject_1_Walking_PAM6_001_Plot_and_Store_Rep_2.12.CSV';EventFrames (2, : ) =[1.54 $\boldsymbol{1}$ 444679795621 739];

name11='Subject_1_Walking_PAM6_001_Plot_and_Store_Rep_3.13.CSv';EventFrames (3, :) =[1.35 $\swarrow$ $419662780605720]$;

name12='Subject_1_Walking_PAM6_001_Plot_and_Store_Rep_4.14.CSv';EventFrames (4, :)=[1.258 $\boldsymbol{}$ $4246627856017 \overline{20}]$;

name13='Subject_1_Walking_PAM6_001_Plot_and_Store_Rep_5.15.CSv';EventFrames (5,:)=[1.457 $459704825643 \overline{7} \overline{6} 5]$;

name14='Subject_1_Walking_PAM6_001_Plot_and_Store_Rep_6.16.CSV'; EventFrames (6, :) =[1.258 $422649769591710]$;

name15='Subject_1_Walking_PAM6_001_Plot_and_Store_Rep_7.17.CSV';EventFrames (7, : ) =[1.14 $\boldsymbol{L}$ $388627744565686]$;

OpenFilterAndProcess 7 (name1, name2, name3, name4, name5, name6, name 7, name8, name9, name10, $\boldsymbol{k}$

name11, name12, name13, name14, name15, EventFrames, numberofpoints, title1) ;

titlel='Walking Exo Elastic';

EventFrames=zeros $(7,6)$;

\%emg frame (header already subtracted, MoCap initial frame, R heel heel, L

:heel heel

name9='S2_Elastic_Plot_and_Store_Rep_1.32.CSV'; EventFrames(1, :)=[1.404 $141391499450 \swarrow$

$558]$;

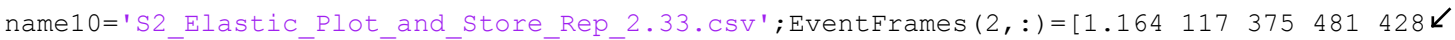
537];

name11='S2_Elastic_Plot_and_Store_Rep_3.34.CSV'; EventFrames(3, :)=[1.382 $139381490439 \swarrow$ 546];

name12='S2_Elastic_Plot_and_Store_Rep_4.35.CSv'; EventFrames (4, :)=[1.624 $150393500449 \boldsymbol{\swarrow}$ $558]$;

name14='S2_Elastic_Plot_and_Store_Rep_5.36.CSV';EventFrames (6, :)=[1.209 $124375485435 \swarrow$ 543];

name15='S2_Elastic_Plot_and_Store_Rep_6.37.CSV'; EventFrames(7,:)=[1.188 $118376482432 \swarrow$ 542]; 


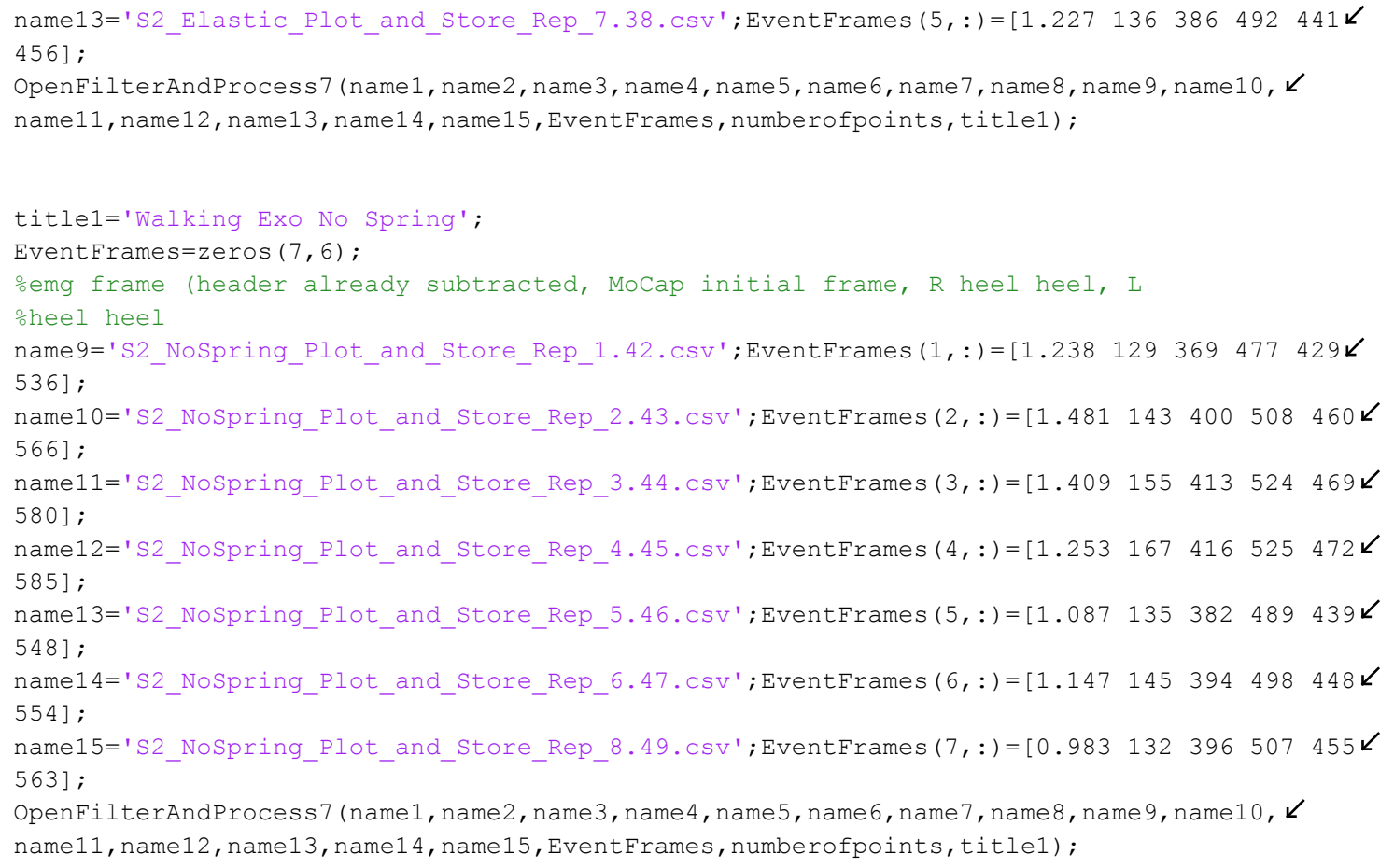




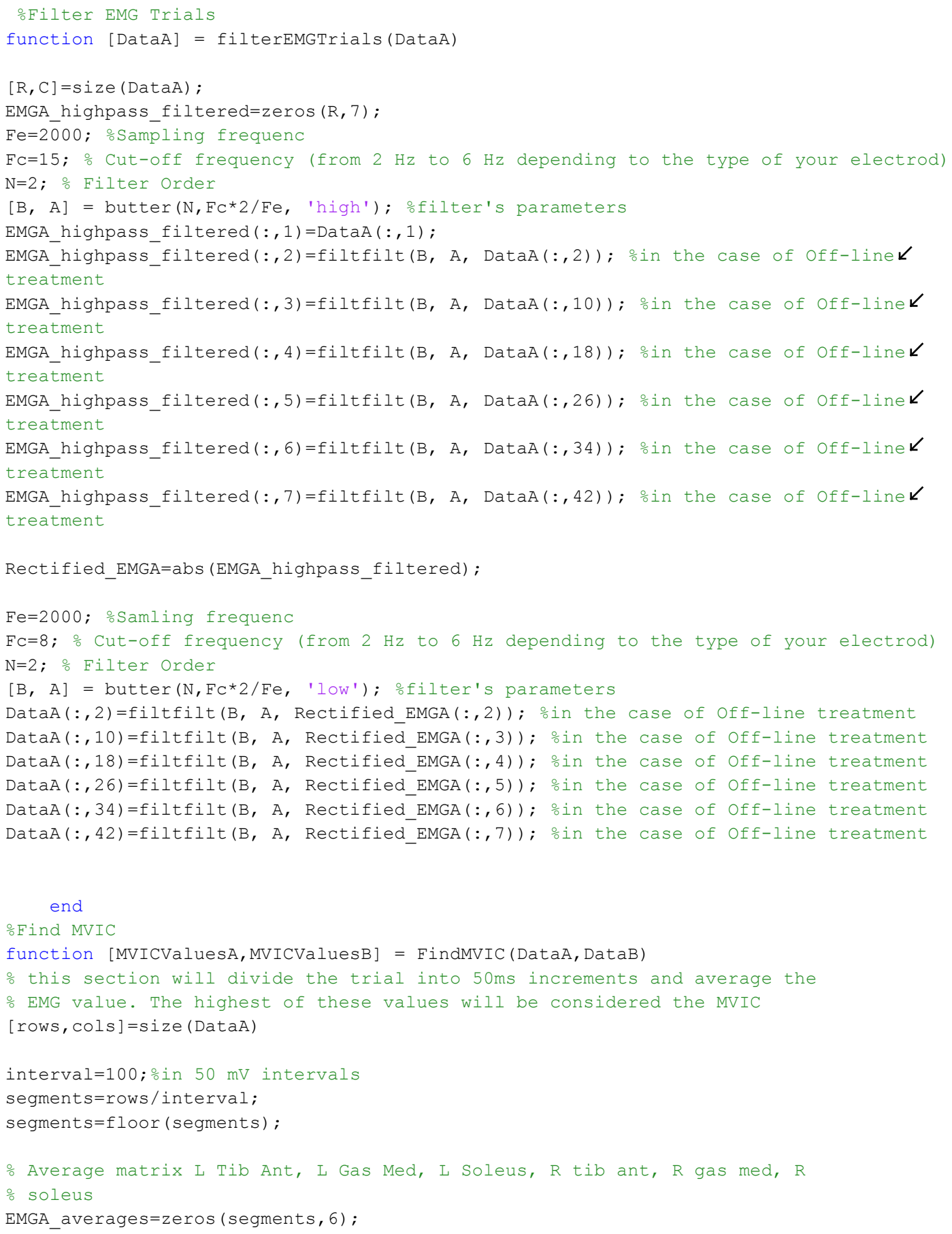




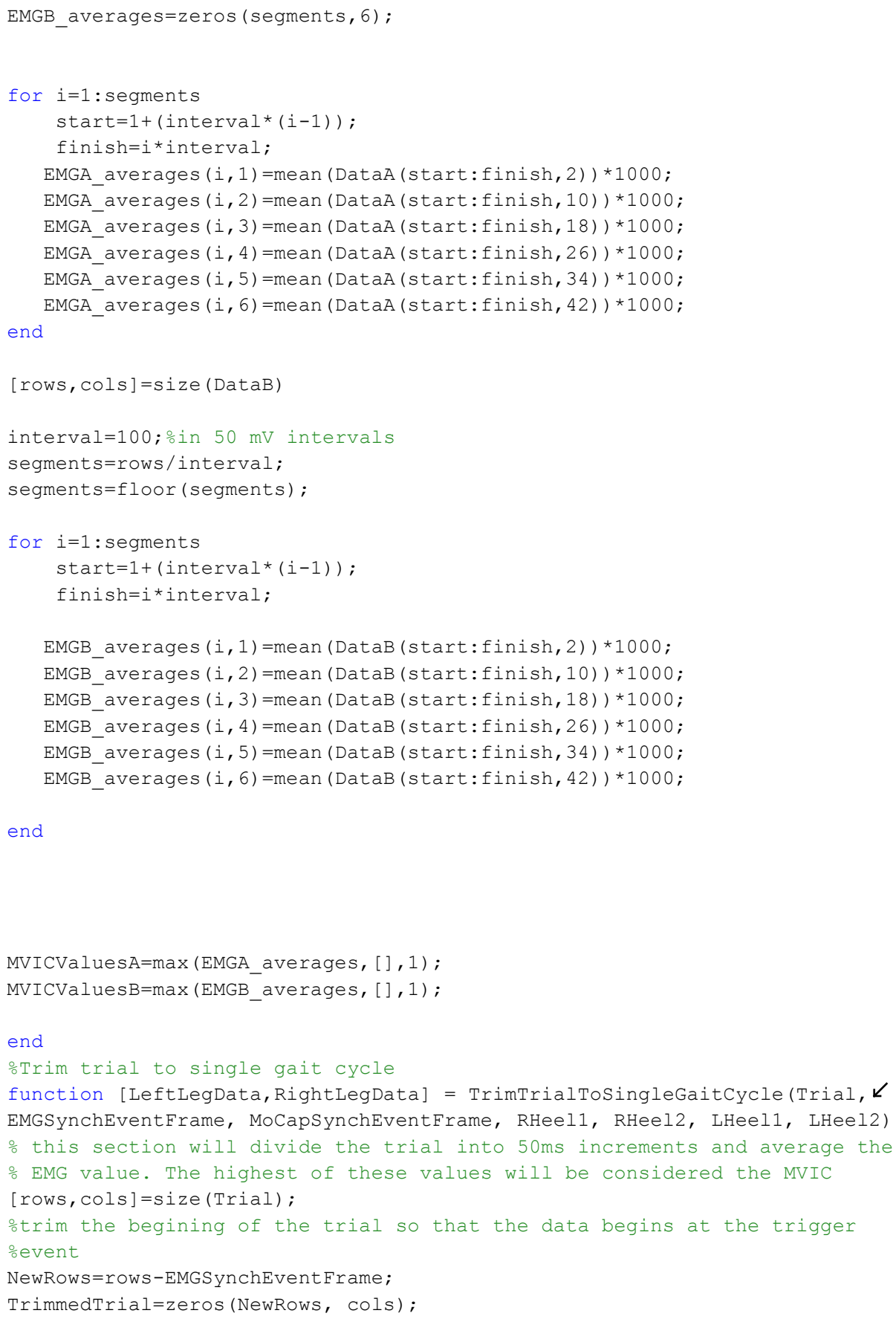




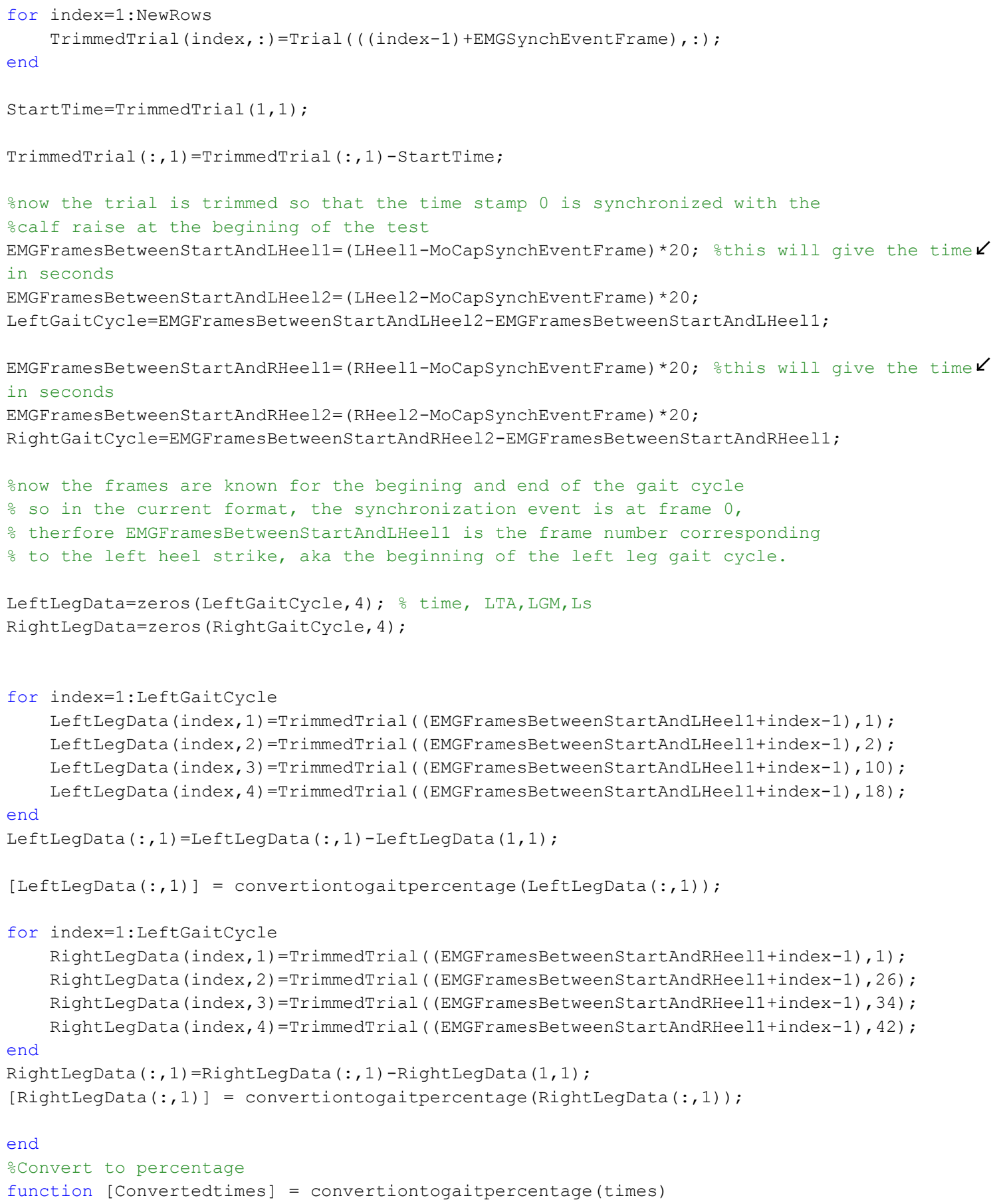




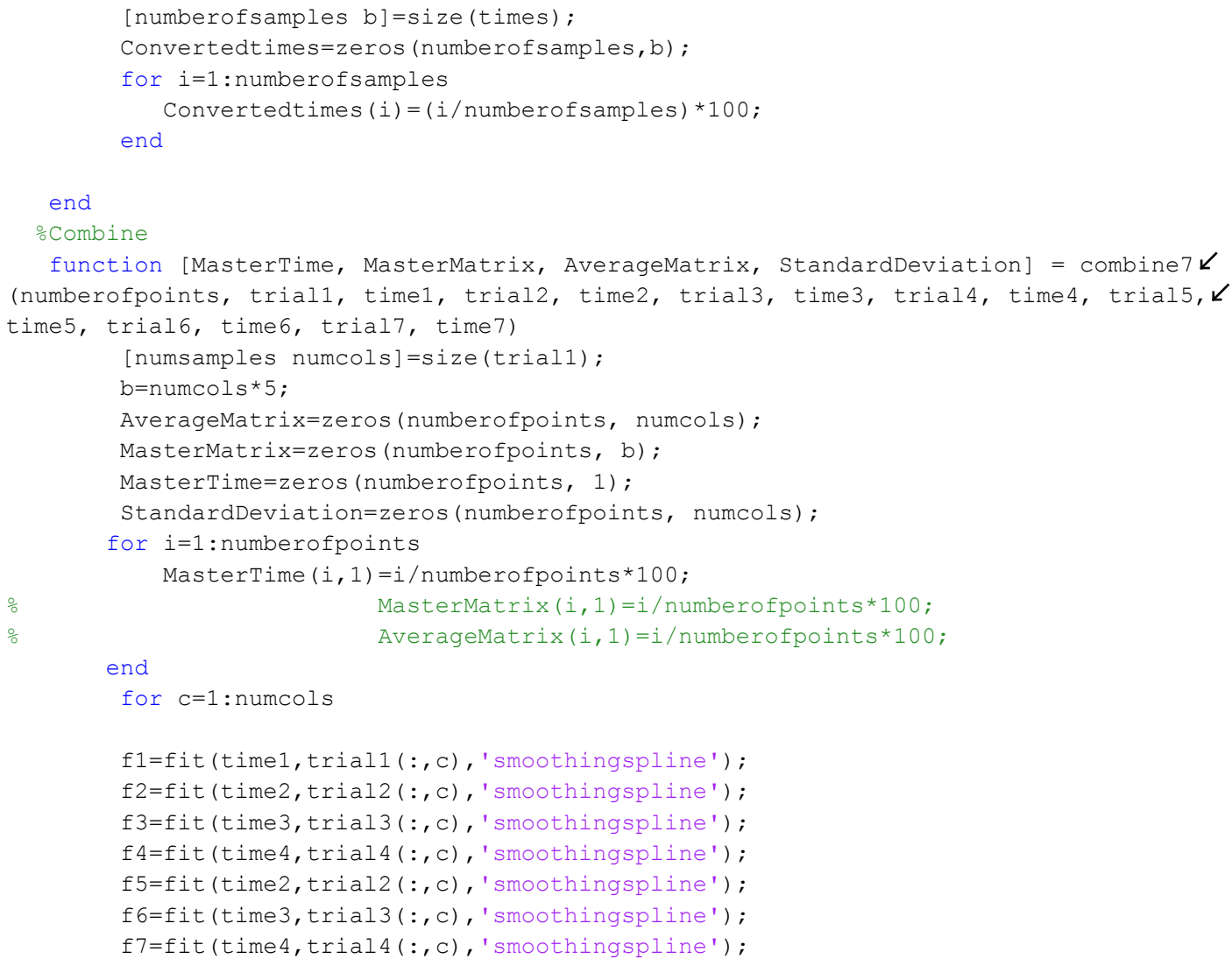









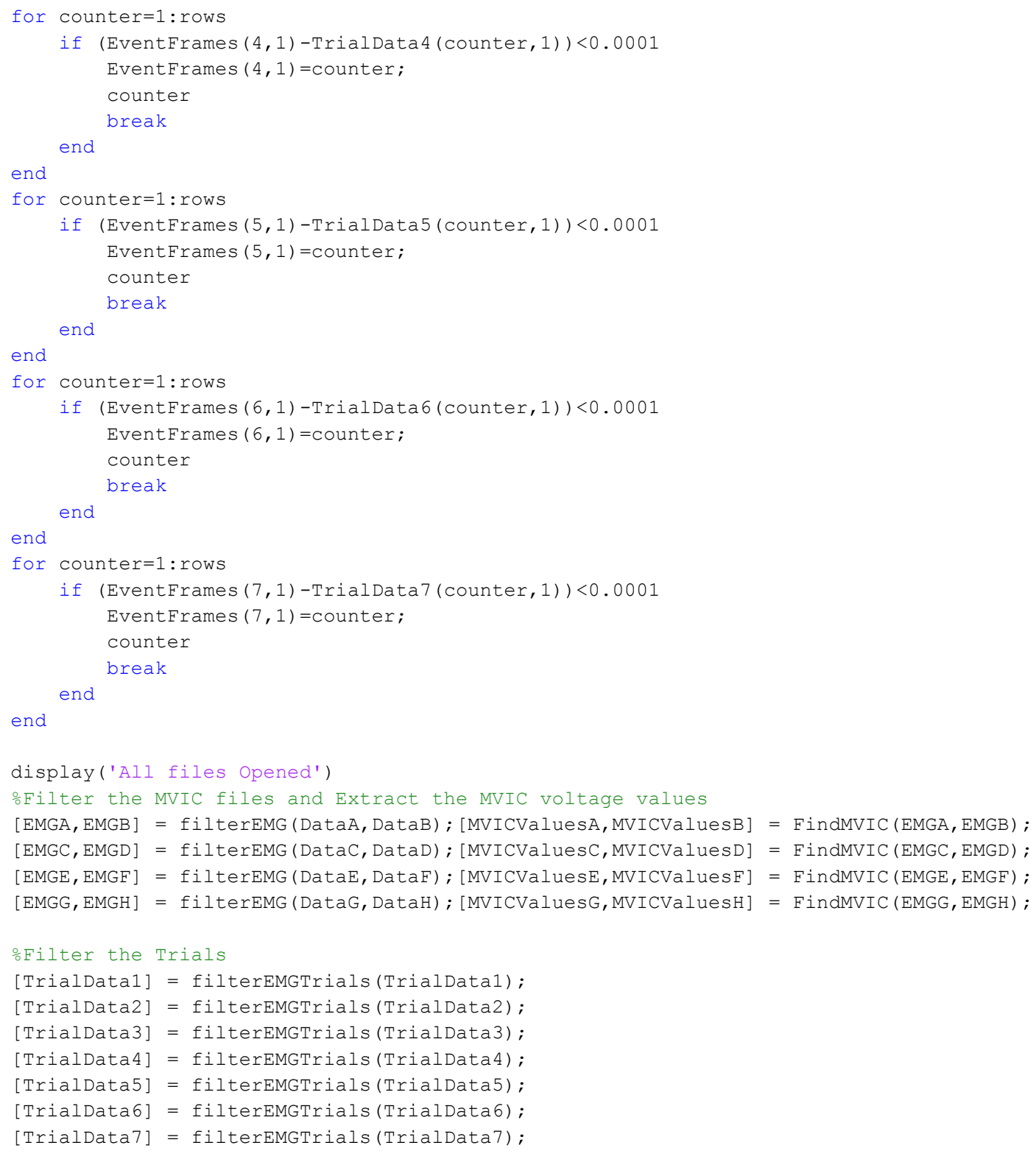

oValues are in order LTA LGM LS RTA RGM RS

AllMVICValues=zeros $(6,8)$;

MVICVALUES $=$ zeros $(1,6)$;

for $j=1: 6$

AllMVICValues $(j, 1)=\operatorname{MVICValuesA}(1, j)$; 
AllMVICValues $(j, 2)=\operatorname{MVICValuesB}(1, j)$; AllMVICValues $(j, 3)=\operatorname{MVICValuesC}(1, j)$; AllMVICValues $(j, 4)=\operatorname{MVICValuesD}(1, j)$; AllMVICValues $(j, 5)=\operatorname{MVICValuesE}(1, j)$; AllMVICValues $(j, 6)=\operatorname{MVICValuesF}(1, j)$; AllMVICValues $(j, 7)=\operatorname{MVICValuesG}(1, j)$; AllMVICValues $(j, 8)=\operatorname{MVICValuesH}(1, j)$; $\operatorname{MVICVALUES}(1, j)=\max (\operatorname{AllmVICValues}(j,:))$; end

display('MVIC Values found')

o Normalizes using the MVIC values

for $u=1: 6$

TrialDatal $(:, 2+((u-1) * 8))=\operatorname{TrialDatal}(:, 2+((u-1) * 8)) * 1000 / \operatorname{MV} \operatorname{CCVALUES}(1, u)$; TrialData $(:, 2+((u-1) * 8))=\operatorname{TrialData} 2(:, 2+((u-1) * 8)) * 1000 / \operatorname{MV} \operatorname{covaluES}(1, u) ;$ TrialData3 $(:, 2+((u-1) * 8))=\operatorname{TrialData} 3(:, 2+((u-1) * 8)) * 1000 / \operatorname{MV} \operatorname{covaluES}(1, u)$ TrialData $4(:, 2+((u-1) * 8))=\operatorname{TrialData} 4(:, 2+((u-1) * 8)) * 1000 / \operatorname{MVICVALUES}(1, u)$; TrialData5 $(:, 2+((u-1) * 8))=\operatorname{TrialData} 5(:, 2+((u-1) * 8)) * 1000 / \operatorname{MV} \operatorname{CCVALUES}(1, u) ;$ TrialData6 $(:, 2+((u-1) * 8))=\operatorname{TrialData} 6(:, 2+((u-1) * 8)) * 1000 / \operatorname{MV} \operatorname{CCVALUES}(1, u)$; TrialData7 $(:, 2+((u-1) * 8))=\operatorname{TrialData} 7(:, 2+((u-1) * 8)) * 1000 / \operatorname{MV} \operatorname{CCVALUES}(1, u) ;$ end

\%(Trial, EMGSynchEventFrame, MoCapSynchEventFrame, RHeel1, RHeel2, LHeel1, LHeel2 [LeftTrial1,RightTrial1] = TrimTrialToSingleGaitCycle(TrialDatal, EventFrames $(1,1), \swarrow$ EventFrames(1,2), EventFrames(1,3), EventFrames(1,4), EventFrames(1,5), EventFrames $\swarrow$ $(1,6))$;

[LeftTrial2,RightTrial2] = TrimTrialToSingleGaitCycle(TrialData2, EventFrames $(2,1), \swarrow$ EventFrames (2,2), EventFrames (2,3), EventFrames $(2,4)$, EventFrames $(2,5)$, EventFrames $\swarrow$ $(2,6))$;

[LeftTrial3,RightTrial3] = TrimTrialToSingleGaitCycle(TrialData3, EventFrames $(3,1), \boldsymbol{K}$ EventFrames(3,2), EventFrames (3,3), EventFrames (3,4), EventFrames $(3,5)$, EventFrames $\swarrow$ $(3,6))$;

[LeftTrial4,RightTrial4] = TrimTrialToSingleGaitCycle(TrialData4, EventFrames(4,1), EventFrames(4,2), EventFrames(4,3), EventFrames (4,4), EventFrames $(4,5)$, EventFrames $\swarrow$ $(4,6))$;

[LeftTrial5,RightTrial5] = TrimTrialToSingleGaitCycle(TrialData5, EventFrames $(5,1), \swarrow$ EventFrames(5,2), EventFrames(5,3), EventFrames(5,4), EventFrames(5,5), EventFrames $\swarrow$ $(5,6))$;

[LeftTrial6,RightTrial6] = TrimTrialToSingleGaitCycle(TrialData6, EventFrames $(6,1), \boldsymbol{l}$ EventFrames (6,2), EventFrames (6,3), EventFrames (6,4), EventFrames $(6,5)$, EventFrames $\swarrow$ $(6,6))$;

[LeftTrial7,RightTrial7] = TrimTrialToSingleGaitCycle(TrialData7, EventFrames $(7,1), \swarrow$ EventFrames(7,2), EventFrames(7,3), EventFrames(7,4), EventFrames(7,5), EventFrames $\swarrow$ $(7,6))$;

[MasterTimeLeftTA, MasterMatrixLeftTA, AverageMatrixLeftTA, StandardDeviationLeftTA] $=\boldsymbol{L}$ combine7 (numberofpoints, LeftTrial1 $(:, 2), \operatorname{LeftTriall}(:, 1), \operatorname{LeftTrial2}(:, 2), \operatorname{LeftTrial2}(:, \boldsymbol{l}$ 1), LeftTrial3(:,2), LeftTrial3(:,1), LeftTrial4(:,2), LeftTrial4(:,1), LeftTrial5(:,2), 
LeftTrial5 (:,1), LeftTrial6(:,2), LeftTrial6(:,1), LeftTrial7(:,2), LeftTrial7 (:,1)) ; [MasterTimeRightTA, MasterMatrixRightTA, AverageMatrixRightTA, StandardDeviationRightTA] $\boldsymbol{}$ = combine7 (numberofpoints, RightTriall(:,2), RightTrial1(:,1), RightTrial2(:,2), $\swarrow$ RightTrial2(:,1), RightTrial3(:,2), RightTrial3(:,1), RightTrial4(:,2), RightTrial4(:,1), RightTrial5(:,2), RightTrial5(:,1), RightTrial6(:,2), RightTrial6(:,1), RightTrial7(:,2), $\swarrow$ RightTrial7 $(:, 1))$;

[MasterTimeLeftGM, MasterMatrixLeftGM, AverageMatrixLeftGM, StandardDeviationLeftGM] = $\swarrow$

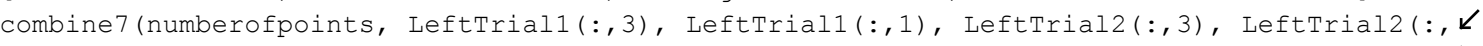
1), LeftTrial3(:,3), LeftTrial3(:,1), LeftTrial4(:,3), LeftTrial4(:,1), LeftTrial5(:,3), $\swarrow$ LeftTrial5 (:,1), LeftTrial6(:,3), LeftTrial6(:,1), LeftTrial7 (:,3), LeftTrial7 (:,1)) ; [MasterTimeRightGM, MasterMatrixRightGM, AverageMatrixRightGM, StandardDeviationRightGM] $\swarrow$ = combine7 (numberofpoints, RightTriall(:,3), RightTrial1(:,1), RightTrial2(:,3), RightTrial2 (:, 1), RightTrial3 (:,3), RightTrial3(:,1), RightTrial4(:,3), RightTrial4(:,1), $\swarrow$ RightTrial5(:,3), RightTrial5(:,1), RightTrial6(:,3), RightTrial6(:,1), RightTrial7(:,3), RightTrial7 $(:, 1))$;

[MasterTimeLeftS, MasterMatrixLeftS, AverageMatrixLefts, StandardDeviationLefts] = $\swarrow$ combine7 (numberofpoints, LeftTrial1 $(:, 4), \operatorname{LeftTrial1}(:, 1), \operatorname{LeftTrial2}(:, 4), \operatorname{LeftTrial2}(:, \mathfrak{l}$ 1), LeftTrial3 $(:, 4), \operatorname{LeftTrial3}(:, 1), \operatorname{LeftTrial4}(:, 4), \operatorname{LeftTrial4}(:, 1), \operatorname{LeftTrial5}(:, 4), \boldsymbol{K}$ LeftTrial5 $(:, 1), \operatorname{LeftTrial6}(:, 4), \operatorname{LeftTrial6}(:, 1), \operatorname{LeftTrial7}(:, 4), \operatorname{LeftTrial7}(:, 1))$; [MasterTimeRightS, MasterMatrixRightS, AverageMatrixRightS, StandardDeviationRightS] = $\swarrow$

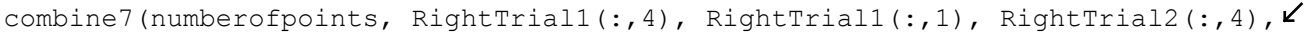
RightTrial2(:,1), RightTrial3(:,4), RightTrial3(:,1), RightTrial4(:,4), RightTrial4(:,1), RightTrial5(:,4), RightTrial5(:,1), RightTrial6(:,4), RightTrial6(:,1), RightTrial7(:,4), RightTrial7 $(:, 1))$;

figure('name', Title);

subplot (2,3,1) ; errorbar (AverageMatrixLeftTA, StandardDeviationLeftTA, 'k') ; title('Left TIB ANT'); ylabel('Fraction of maximum muscle contraction'); $x \mathbf{l a b e l}($ 'Percentage $\boldsymbol{}$ of gait cycle (oㅜㅇ' '); $\operatorname{axis}\left(\left[\begin{array}{llll}0 & 100 & 0 & 1\end{array}\right]\right)$ subplot (2,3,2) ; errorbar (AverageMatrixLeftGM, StandardDeviationLeftGM, 'k' ) ; title('Left Gas Med'); ylabel('Fraction of maximum muscle contraction'); xlabel('Percentage $\boldsymbol{}$ of gait cycle (\%)');

$\operatorname{axis}\left(\left[\begin{array}{llll}0 & 100 & 0 & 1\end{array}\right]\right)$

subplot $(2,3,3)$; errorbar (AverageMatrixLefts, StandardDeviationLefts, 'k') ; title('Left Soleus'); ylabel('Fraction of maximum muscle contraction');xlabel('Percentage of gait cycle ( $\left.\left.\frac{\circ}{0}\right)^{\prime}\right)$; axis ( [ $\left.\left.\begin{array}{llll}0 & 100 & 0 & 1\end{array}\right]\right)$ subplot (2, 3,4); errorbar (AverageMatrixRightTA, StandardDeviationRightTA, 'k') ; title('Right TIB ANT'); ylabel ('Fraction of maximum muscle contraction'); $\mathrm{xlabel} \boldsymbol{\swarrow}$ ('Percentage of gait cycle (\%)'); $\operatorname{axis}\left(\left[\begin{array}{llll}0 & 100 & 0 & 1\end{array}\right]\right)$ subplot $(2,3,5)$; errorbar (AverageMatrixRightGM, StandardDeviationRightGM, 'k' ) ; title('Right Gas Med'); ylabel('Fraction of maximum muscle contraction'); xlabel $\boldsymbol{~}$ ('Percentage of gait cycle (\%)');

$\operatorname{axis}\left(\left[\begin{array}{llll}0 & 100 & 0 & 1\end{array}\right]\right)$

subplot $(2,3,6)$; errorbar (AverageMatrixRightS, StandardDeviationRightS, 'k' ) ; 
04/05/17 2:39 PM C:\User...\EMG MVIC normalized EMG s2.m

12 of 12

title('Right Soleus'); ylabel('Fraction of maximum muscle contraction'); xlabel('Percentage $\boldsymbol{}$ of gait cycle (\%)' ');

axis $\left(\left[\begin{array}{llll}0 & 100 & 0 & 1\end{array}\right]\right)$

end 